Issued by Sandia National Laboratories, operated for the United States Department of Energy by Sandia Corporation.

NOTICE: This report was prepared as an account of work sponsored by an agency of the United States Government. Neither the United States Government, nor any agency thereof, nor any of their employees, nor any of their contractors, subcontractors, or their employees, make any warranty, express or implied, or assume any legal liability or responsibility for the accuracy, completeness, or usefulness of any information, apparatus, product, or process disclosed, or represent that its use would not infringe privately owned rights. Reference herein to any specific commercial product, process, or service by trade name, trademark, manufacturer, or otherwise, does not necessarily constitute or imply its endorsement, recommendation, or favoring by the United States Government, any agency thereof, or any of their contractors or subcontractors. The views and opinions expressed herein do not necessarily state or reflect those of the United States Government, any agency thereof, or any of their contractors.

Printed in the United States of America. This report has been reproduced directly from the best available copy.

Available to DOE and DOE contractors from

Office of Scientific and Technical Information

P.O. Box 62

Oak Ridge, TN 37831

Prices available from (703) 605-6000

Web site: http://www.ntis.gov/ordering.htm

Available to the public from

National Technical Information Service

U.S. Department of Commerce

5285 Port Royal Rd

Springfield, VA 22161

NTIS price codes

Printed copy: A12

Microfiche copy: A01

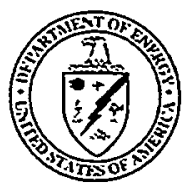




\section{DISCLAIMER}

Portions of this document may be illegible in electronic image products. Images are produced from the best available original document. 
SAND99-2706

Unlimited Release

Printed October 1999

\title{
Space -Variant Post-Filtering for Wavefront Curvature Correction in Polar-Formatted Spotlight-Mode SAR Imagery
}

\author{
Neall E. Doren \\ Analysis Department II \\ Sandia National Laboratories \\ Albuquerque, NM 87185
}

\begin{abstract}
Wavefront curvature defocus effects occur in spotlight-mode SAR imagery when reconstructed via the well-known polar-formatting algorithm (PFA) under certain imaging scenarios. These include imaging at close range, using a very low radar center frequency, utilizing high resolution, and/or imaging very large scenes. Wavefront curvature effects arise from the unrealistic assumption of strictly planar wavefronts illuminating the imaged scene. This dissertation presents a method for the correction of wavefront curvature defocus effects under these scenarios, concentrating on the generalized, squint-mode imaging scenario and its computational aspects. This correction is accomplished through an efficient one-dimensional, image domain filter applied as a post-processing step to PFA. This post-filter, referred to as SVPF, is precalculated from a theoretical derivation of the wavefront curvature effect and varies as a function of scene location. Prior to SVPF, severe restrictions were placed on the imaged scene size in order to avoid defocus effects under these scenarios when using PFA. The SVPF algorithm eliminates the need for scene size restrictions when wavefront curvature effects are present, correcting for wavefront curvature in broadside as well as squinted collection modes while imposing little additional computational penalty for squinted images.

This dissertation covers the theoretical development, implementation and analysis of the generalized, squint-mode SVPF algorithm (of which broadside-mode is a special case) and provides examples of its capabilities and limitations as well as offering guidelines for maximizing its computational efficiency. Tradeoffs between the PFA/SVPF combination and other spotlight-mode SAR image formation techniques are discussed with regard to computational burden, image quality, and imaging geometry constraints. It is demonstrated that other methods fail to exhibit a clear computational advantage over polar-formatting in conjunction with SVPF. This research concludes that PFA in conjunction with SVPF provides a computationally efficient spotlight-mode image formation solution that solves the wavefront curvature problem for most standoff distances and patch sizes, regardless of squint, resolution or radar center frequency. Additional advantages are that SVPF is not iterative and has no dependence on the visual contents of the scene, resulting in a deterministic computational complexity which typically adds only thirty percent to the overall image formation time.
\end{abstract}


Neall Evan Doren

Condidate

Electrical and Computer Engineering

Department

This dissertation is approved, and it is acceptable in quality and form for publication on microfilm:

Approved by the Dissertation Committee:

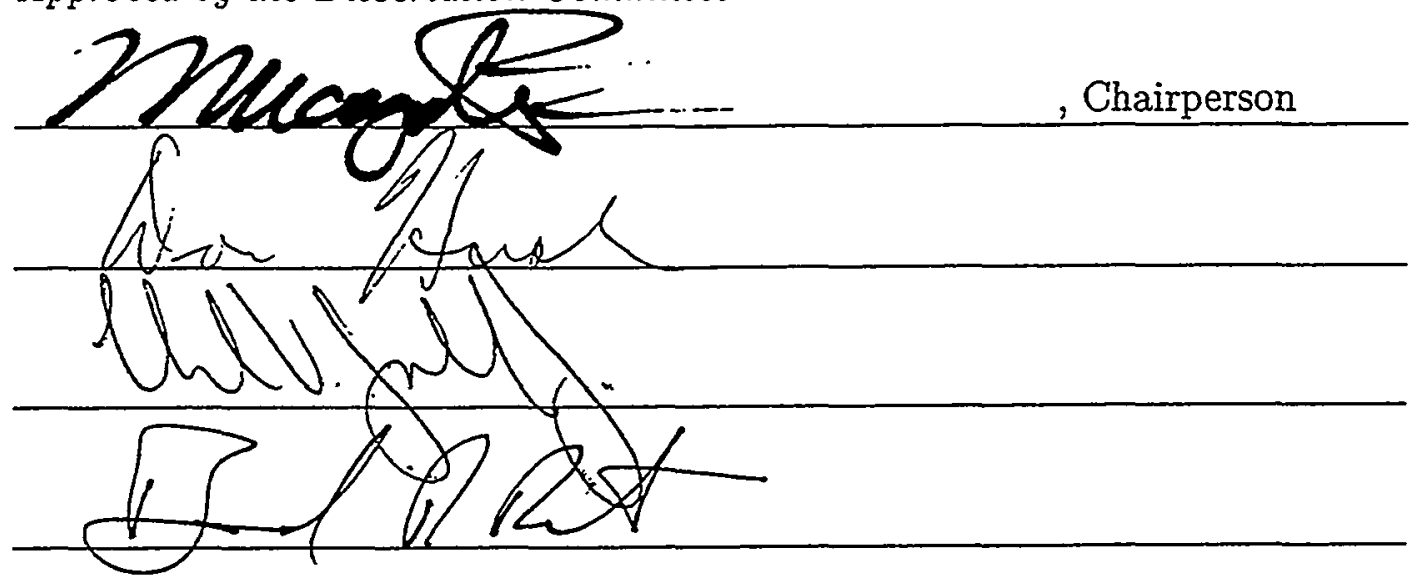

Accepted:

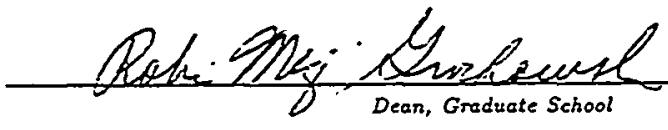

OCT 121999

Date 
This page intentionally left blank 
Space-Variant Post-Filtering for Wavefront Curvature Correction in Polar-Formatted Spotlight-Mode SAR Imagery

by

\section{Neall Evan Doren}

B.S., University of Southern Colorado, 1983

M.S., Electrical Engineering, University of New Mexico, 1991

\section{DISSERTATION}

Submitted in Partial Fulfillment of the

Requirements for the Degree of

Doctor of Philosophy

Engineering

The University of New Mexico

Albuquerque, New Mexico

December, 1999 
(C)1999, Neall Evan Doren 


\section{Dedication}

To my parents, Carl and Horty Doren, for their support, encouragement and love.

To my wife, Maribeth, for her countless sacrifices and endless patience and understanding.

To my favorite high school teacher, Terry J. Cyprian, whose faith in me some twenty years ago will never be forgotten. Teachers do make a difference.

"Never, never, never give up" - Winston Churchill 


\section{Acknowledgements}

I would like to thank my advisor, Professor Neeraj Magotra, for his encouragement and continued support of my work. I would also like to thank my manager, Dr. Jack Jakowatz, for unselfishly offering his time and expert technical advice to this effort. He has been an inspiration both professionally and spiritually, and he originally proposed this interesting and valuable research topic. I am also grateful to my dissertation committee for their time and efforts.

The generous educational benefits provided by Sandia Laboratories ${ }^{1}$, as well as easy access to their leading-edge Instructional Television Services (ITV), allowed me the opportunity to pursue my dreams. I am grateful to my managers past and present: Dr. Jakowatz, Dr. Larry Stotts, Dr. Jim Baremore and Ms. Dori Ellis, for allowing me to utilize these company benefits and helping me to realize my full potential.

Additional thanks are extended to Ms. Julie Kesti of Sandia Labs' technical library for her literature searches and prompt document delivery, and to the "Lunatic Fringe," a group of talented SAR engineers including Drs. Dennis Ghiglia, Paul Thompson, and Dan Wahl, who were instrumental to my success. Dr. Wahl's suggestions became the basis of many of my computer simulations and he authored the RMA and FReD simulations used in the comparisons of Chapter 6. Dr. Thompson aided in my understanding of the theoretical mathematical models and described to me the mathematics of the broadside phase model. Dr. Ghiglia provided insight into the effects of phase errors on IPR broadening and first derived the formulas of Appendix A. A note of appreciation goes to Mrs. Joan Lillie, whose administrative expertise and kindness kept me from falling through the cracks. Furthermore, my thanks to Dr. James W. Howse, my close friend and fellow graduate student, and Dr. Ireena Erteza, who (gently) prodded me and kept me inspired, excited and motivated through the good times and bad. Also, to Dr. Don Hush, who provided so much encouragement and support over the past 15 years, in addition to serving on my committee. Last but not least, a big thanks to Mrs. Erika Papp, who kept me sane through thick and thin. Many thanks to all.

\footnotetext{
${ }^{1}$ This work was supported by the US Department of Energy under contract No. DEAC04-94AL85000. Sandia is a multiprogram laboratory operated by Sandia Corporation, a Lockheed Martin Company, for the United States Department of Energy.
} 


\section{Space-Variant Post-Filtering for Wavefront Curvature Correction in Polar-Formatted Spotlight-Mode SAR Imagery}

by

Neall Evan Doren

\section{ABSTRACT OF DISSERTATION}

Submitted in Partial Fulfillment of the

Requirements for the Degree of

Doctor of Philosophy

Engineering

The University of New Mexico

Albuquerque, New Mexico

December, 1999 
This page intentionally left blank 


\title{
Space-Variant Post-Filtering for Wavefront Curvature Correction in Polar-Formatted Spotlight-Mode SAR Imagery
}

by

\section{Neall Evan Doren}

B.S., University of Southern Colorado, 1983

M.S., Electrical Engineering, University of New Mexico, 1991

Ph.D. Electrical Engineering, University of New Mexico, 1999

\begin{abstract}
Wavefront curvature defocus effects occur in spotlight-mode SAR imagery when reconstructed via the well-known polar-formatting algorithm (PFA) under certain imaging scenarios. These include imaging at close range, using a very low radar center frequency, utilizing high resolution, and/or imaging very large scenes. Wavefront curvature effects arise from the unrealistic assumption of strictly planar wavefronts illuminating the imaged scene. This dissertation presents a method for the correction of wavefront curvature defocus effects under these scenarios, concentrating on the generalized, squint-mode imaging scenario and its computational aspects. This correction is accomplished through an efficient one-dimensional, image domain spacevariant filter applied as a post-processing step to PFA. This space-variant post-filter, referred to as SVPF, is precalculated from a theoretical derivation of the wavefront curvature effect and varies as a function of scene location. Prior to SVPF, severe
\end{abstract}


restrictions were placed on the imaged scene size in order to avoid defocus effects under these scenarios when using PFA. The SVPF algorithm eliminates the need for scene size restrictions when wavefront curvature effects are present, correcting for wavefront curvature in broadside as well as squinted collection modes while imposing little additional computational penalty for squinted images.

This dissertation covers the theoretical development, implementation and analysis of the generalized, squint-mode SVPF algorithm (of which broadside-mode is a special case) and provides examples of its capabilities and limitations as well as offering guidelines for maximizing its computational efficiency. Tradeoffs between the PFA/SVPF combination and other spotlight-mode SAR image formation techniques are discussed with regard to computational burden, image quality, and imaging geometry constraints. It is demonstrated that other methods fail to exhibit a clear computational advantage over polar-formatting in conjunction with SVPF. This research concludes that PFA in conjunction with SVPF provides a computationally efficient spotlight-mode image formation solution that solves the wavefront curvature problem for most standoff distances and patch sizes, regardless of squint, resolution or radar center frequency. Additional advantages are that SVPF is not iterative and has no dependence on the visual contents of the scene, resulting in a deterministic computational complexity which typically adds only thirty percent to the overall image formation time. 


\section{Contents}

List of Figures

xiii

List of Tables

xvi

Glossary

xvii

1 Introduction

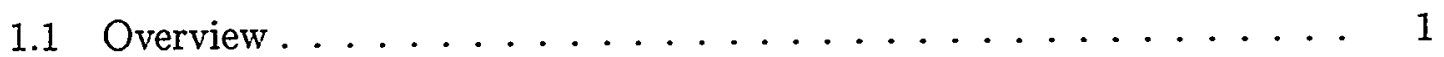

1.2 Introducing the Space-Variant Post-Filter $\ldots \ldots \ldots \ldots \ldots$

1.3 Alternative Spotlight-Mode Image Formation Methods . . . . . . 11

1.4 Dissertation Objectives . . . . . . . . . . . . 15

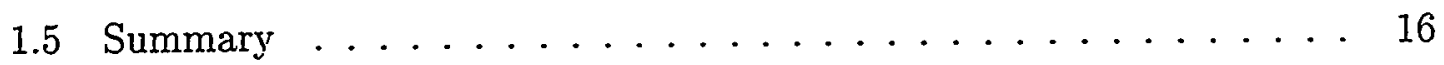

1.6 Organization of this Dissertation $\ldots \ldots \ldots \ldots \ldots$

2 Aperture Synthesis, Tomography and Polar-Formatting 23

2.1 The Range Resolution Problem . . . . . . . . . . . 24

2.2 Cross-Range Resolution Via Synthetic Aperture . . . . . . . 26 
2.2.1 Synthetic Aperture Radar in Stripmap-Mode . . . . . . . 28

2.2.2 Synthetic Aperture Radar in Spotlight-Mode . . . . . . . . 29

2.3 Spotlight-Mode Tomography and Polar-

Formatted Data . . . . . . . . . . . . . . . 32

2.4 The Polar-Format Algorithm . . . . . . . . . . . . . . 42

3 The Planar Wavefront Assumption in Polar-Formatted Imagery 51

3.1 The Planar Wavefront Assumption - A Limitation of the Tomographic Paradigm .......................... 52

3.2 The Cost of Ignoring Spherical Wavefronts . . . . . . . . . . 54

3.2 .1 Geometric Distortion . . . . . . . . . . . 55

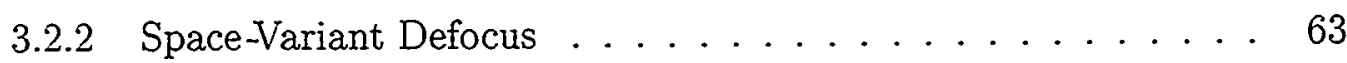

3.3 Scene Size Limitations Due to Wavefront Curvature Defocus Effects . 69

3.4 The Space-Variant Post-Filtering Approach to Wavefront Curvature Correction ........................... 74

4 Analysis of Phase Errors Arising From Wavefront Curvature $\quad 77$

4.1 Point Target Contribution to Fourier Domain Reflectivity Function - 79

4.2 The Phase Return From a Point Target . . . . . . . . . . . 85

4.2.1 Broadside Phase Error Modeling ........... 85

4.2.2 Phase of Video Signal with Respect to Imaging Geometry . 90

4.2.3 Synthetic Target Generation . . . . . . . . . . . 93

4.3 Polynomial Phase Term Representation . . . . . . . . . . . . 96 
4.3.1 Linear Phase Terms . . . . . . . . . . . . . . . . . 97

4.3.2 Decomposition of Linear Phase Terms . . . . . . . . 104

4.3.3 Quadratic Phase Terms............... 108

4.4 Generalized Phase Error Model for all Squint Modes . . . . . . . 111

4.4 .1 Squint-Mode Geometry . . . . . . . . . . . . . . 111

4.4.2 Polynomial Phase Terms for Squint-Mode . . . . . . 120

4.4.3 Quadratic Phase Error Term for Squinted Collections . . . . 125

4.5 Summary of Phase Error Analysis . . . . . . . . . . . 131

5 Space-Variant Post-Filter Design

5.1 The Concept of Wavefront Curvature Correction Via Space-Variant Post-Filtering . . . . . . . . . . . . . 136

5.2 Space Invariance - Scope of Application $\ldots \ldots \ldots \ldots$. . . . 139

5.3 Accounting For Geometric Distortion . . . . . . . . . 148

5.4 Filter Overlap and Width $\ldots \ldots \ldots \ldots \ldots \ldots \ldots$

5.5 Filter Displacement $\ldots \ldots \ldots \ldots \ldots \ldots \ldots \ldots$

6 Performance of the Space-Variant Post-Filter 167

6.1 Quality of Refocus . . . . . . . . . . . 168

6.2 Computational Complexity . . . . . . . . . . . . 177

6.2 .1 General Complexity . . . . . . . . . . . . . 177

6.2.2 Adjusting Filter Parameters to Reduce Computational Cost . 182 
6.2.3 Additional Computational Burden of Post-Filtering to PolarFormatting . . . . . . . . . . . . . . . 184

6.3 Effect of Squint on the Computational Burden of Space-Variant PostFiltering . . . . . . . . . . . . . . . 185

6.4 Computer Simulation Results . . . . . . . . . . . . . . 192

6.4.1 Choice of Algorithms . . . . . . . . . . . . . . . 192

6.4.2 Benchmark Results . . . . . . . . . . . . . . . . . 193

6.4 .3 Observations . . . . . . . . . . . . . 195

$\begin{array}{lll}7 \text { Conclusion } & 199\end{array}$

7.1 Summary . . . . . . . . . . . . . . . 199

7.2 Contributions of this Dissertation . . . . . . . . . . 201

7.3 Future Work . . . . . . . . . . . . . . . . 204

A IPR Broadening Due to Quadratic Phase Errors 209

A.1 IPR Broadening Based on Spectral Width $\ldots \ldots \ldots \ldots . \ldots 213$

A.2 Spatial Domain IPR Broadening . . . . . . . . . . 215

$\begin{array}{ll}\text { References } & 217\end{array}$ 


\section{List of Figures}

1.1 Stripmap-Mode SAR Imaging Geometry $\ldots \ldots \ldots . . . . .2$

1.2 Spotlight-Mode SAR Imaging Geometry for Polar-Formatting . . . 3

1.3 Basic Steps to the Polar-Format Processing Algorithm _ . . . . 6

1.4 Range Curvature of CRP During Spotlight-Mode Collection $\ldots . \quad 7$

1.5 Geometric Image Distortion Induced by Wavefront Curvature . . 8

1.6 Simulated Wavefront Curvature Defocus Effects Before and After Removal ....................... 9

1.7 Squint Modes for Spotlight-Mode SAR Collections . . . . . . . . 17

2.1 Beamwidth, Aperture Extent and Patch Radius in Spotlight-Mode .

2.2 Projectional View of X-ray Tomography $\ldots \ldots \ldots \ldots \ldots$

2.3 Slant Plane Fourier-Domain Samples and Frequency Extent _. . 37

2.4 Slant Plane Fourier-Domain Samples and Ground Plane Projection for Spotlight-Mode . . . . . . . . . . . . . . 40

2.5 Polar-Format Processing Algorithm - Detailed Steps . . . . . . 45 
3.1 Actual Spherical Wavefront Curvature Versus the Planar Assumption for Spotlight-Mode SAR ............... 54

3.2 Broadside Model for Derivation of Geometric Distortion When Neglecting Spherical Wavefront Characteristics ......... 56

3.3 Geometric Image Distortion From Wavefront Curvature - Broadside Collection ......................... 59

3.4 Simulated Geometric Distortion Effects of Wavefront Curvature . . 60

3.5 Actual Ku-Band SAR Image of Sled Track With Wavefront Curvature Distortion (1 Ft. Res.) . . . . . . . . . . . 61

3.6 Simulated Wavefront Curvature Effects Before and After Removal Severely Defocused Region . . . . . . . . . . . . . 67

3.7 Simulated Wavefront Curvature Effects Before and After Removal Slightly Defocused Region . . . . . . . . . . . 68

3.8 Cross-Range Defocus for Various Values of Peak Quadratic Phase . 70

4.1 Slant Plane Imaging Geometry for Point Target Return . . . . . . . 82

4.2 Broadside Geometric Model for Derivation of Wavefront Curvature Phase Errors ................... 87

4.3 Aperture Center Geometric Model for Derivation of Wavefront Curvature Phase Errors . . . . . . . . . . . . . . 98

4.4 Squint Angle and Standoff Range Definitions for Spotlight-Mode . . 113

4.5 Geometric Model for Derivation of Squinted-Collection Wavefront Curvature Phase Errors . . . . . . . . . . . . . . 115 
5.1 Wavefront Curvature Correction of Synthetic Point Targets via a Space-Invariant Post-Filter Centered at $\left(x_{0}^{\prime}, y_{0}^{\prime}\right) \ldots \ldots$. . . . . 142

5.2 Space-Invariant Vs. Space-Variant Filtering . . . . . . . . 145

$5.3 \quad$ Filter Overlap, Displacement and Length . . . . . . . . . . 146

5.4 Implementation of Space-Variant Post-Filter for Two Patches . . . . 147

5.5 Effect of Geometric Distortion on Target Position . . . . . . . 150

6.1 L-Band Squint-Mode Collection Geometry with Associated SYNTARG Target Patch (No Wavefront Curvature Correction) . . . . . 171

6.2 Enlarged View: Squint-Mode Point Targets Before and After Refocus

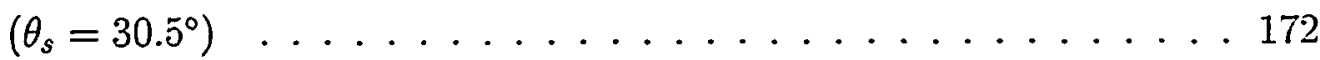

6.3 Refocused L-Band Point Target with Associated IPR Plot (Squinted Collection Geometry, $\left.\theta_{s}=30.5^{\circ}\right) \ldots \ldots 174$

6.4 Refocused L-Band Point Target with Associated IPR Plot (Broadside Collection Geometry) . . . . . . . . . . . 176

6.5 Resolvability of Crowded L-Band Targets (Broadside Collection Ge-

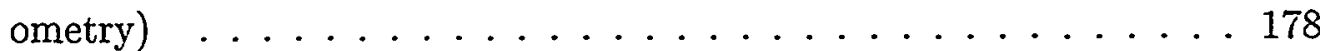

6.6 Post-Filter Operations Count as a Function of Filter Width and

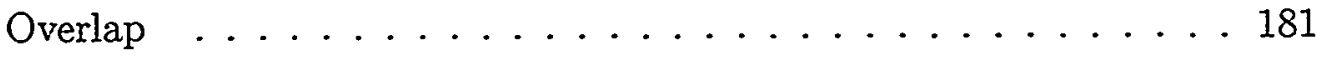

A.1 IPR Response for Point Target with Spatial Bandwidth $\Delta U \ldots$. . 211

A.2 Effects of Quadratic Phase Errors on the Ideal IPR . . . . . . 213 
xvii

\section{List of Tables}

3.1 Range "Sag" at Scene Edge for Ku Band Sled Track Example . . . 61

3.2 Scene Radius Limits (meters) From Wavefront Curvature Quadratic Phase Errors ................... . . 74

$5.1 \quad$ L-Band Parameters for Broadside, Space-Invariant Experiment . . . 141

6.1 Radar Parameters for Workstation-Based Timing Benchmark . . . . 194

6.2 SVPF-Specific Parameters for Workstation-Based Timing Benchmark 194

6.3 Execution Times for Five Algorithms in FORTRAN on a Sun Ultra-2

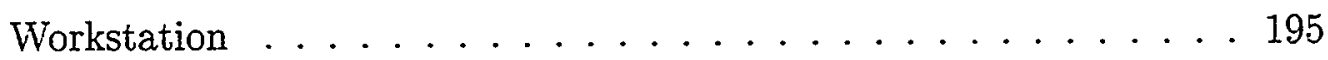




\title{
Glossary
}

\author{
$a_{l m}$ \\ Taylor series coefficients, where $l, m=\{0 . .2\}$ \\ $A_{p}$ \\ Complex-valued scalar denoting the amplitude and phase of a \\ radar return. \\ $A^{T}$ \\ Transpose of phase history data (as done in polar-format algo- \\ rithm). \\ $A_{x}$ \\ Physical width of the antenna in the along-track (azimuth) direc- \\ tion. \\ $B_{c} \quad$ Bandwidth of linear FM chirp. \\ $\beta_{a} \quad$ Cone angle of beam at radar antenna. \\ $C_{f f t} \quad$ Complex operations count for an FFT. \\ $C_{p} \quad$ Overall complex operations count. \\ $C_{p / p i x} \quad$ Complex operations count, per pixel. \\ $C_{f / p i x} \quad$ Floating point operations count, per pixel. \\ $C_{o p t_{f / p i x}} \quad$ Optimal floating point operations count, per pixel. Based on op- \\ timal filter length $m_{\text {opt }}(c)$ for some $c$. \\ CRP Central reference point of a spotlight-mode collection. Typically \\ the scene center.
}


CSA

CWW

$c$

c

$c_{b}, c_{s}$

$D$

$D_{p}$

$d$

$d_{b}, d_{s}$

$d_{\text {opt }}$

$\Delta f_{0}$

$\triangle f_{Q P E}$

$\frac{\Delta f_{Q P E}}{\Delta f_{0}}$

$\triangle_{I P R}$
Chirp scaling algorithm. Simplified RMA image formation via approximation (chirp- $Z$ transform) instead of Stolt interpolation. Continuous wave (non-FM range pulse).

Speed of light.

(Maximum) overlap between adjacent space-variant filter applications.

Overlap between adjacent space-variant filter applications, broadside and squint collection geometries, respectively.

Physical diameter of radar antenna.

Patch diameter of imaged scene.

(Maximum) displacement in cross-range from the previous spacevariant filter application.

Displacement in cross-range from previous space-variant filter application, broadside and squint collection geometries, respectively.

Optimal displacement in cross-range from previous space-variant filter application, yielding lowest operations count for some filter overlap $c$.

$d_{x_{0}^{\prime}, y_{0}^{\prime}}\left(X^{\prime}, Y^{\prime}\right) \quad$ Point target response of $\left(x_{0}^{\prime}, y_{0}^{\prime}\right)$ for the frequency extent $X^{\prime}$ and $Y^{\prime}$.

Nominal spectral width of IPR.

Nominal spectral width of quadratic phase error signal $e^{j \alpha t^{2}}$.

IPR broadening factor.

IPR broadening factor. 
$\Delta n$

$\Delta n_{\epsilon}$

$\Delta \theta$

$\Delta U$

$\Delta U_{x}, \Delta U_{y}$

$\Delta X^{\prime}, \Delta Y^{\prime}$

$\Delta y^{\prime}$

$\delta X^{\prime}$

EM

FMI

FReD

$\mathcal{F}\{\}$

$\stackrel{\mathcal{F}}{\Longleftrightarrow}$

$f_{0}$ or $f_{c}$

$f_{\text {inst }}$

$f_{\hat{x}^{\prime}} X^{\prime}, f_{\hat{y}^{\prime}} Y^{\prime} \quad$ Linear phase error terms in the slant plane, for cross-range and range, respectively.

IPR pixel spread.

IPR pixel spread due to quadratic phase error.

Angular diversity of synthetic aperture.

Spatial bandwidth of impulse response.

Spatial bandwidth of impulse response in cross-range and range, respectively.

Spatial bandwidth (frequency extent) of slant plane impulse response in cross-range and range, respectively.

Amount of geometric distortion in range, at edge of scene (a distance $L$ from the CRP).

Discrete frequency spacing of Fourier transformed image patch.

Electromagnetic (wave).

Frequency modulation.

Frequency domain replication and downsampling algorithm. A newer RMA technique to reduce along-track upsampling in squintmode.

Fourier transform operation.

Fourier transform pair.

Radar center frequency corresponding to wavelength, $\lambda=c / f_{0}$.

Instantaneous frequency of a signal. 
$f_{\widehat{x^{\prime}}} X^{\prime 2}$

$G(u, v)$

$g(x, y)$

IFSAR

IPR

ISAR

$K_{a}$

$k_{0}$

$k_{t}$

$k_{\theta t}$

$\lambda$

$L$

L-Band

LFM

MTRC

$m$
Quadratic cross-range phase error term in the slant plane.

Fourier domain transformation of attenuation function (or ground patch reflectivity function).

Two-dimensional attenuation function (or the complex reflectivity of ground patch).

Interferometric SAR. Interferometry used for terrain height estimation.

Impulse response (function).

Inverse SAR. SAR analysis of rotating objects relative to a fixed sensor (antenna), as based on the tomographic paradigm.

IPR mainlobe broadening factor (in cross-range) due to sidelobe reduction (windowing).

Nominal phase history radius (in units of spatial frequency).

Magnitude of reflected EM range pulse at intra-pulse time $t$.

Specifies a position in the Fourier domain as a function of time, $t$, and angular orientation $\theta$.

Radar wavelength corresponding to center frequency, $f_{0}=c / \lambda$.

Illuminated patch radius, in azimuth.

Foliage penetrating microwave radar $\left(f_{c} \approx 1.25 \mathrm{gHz}\right)$, vulnerable to wavefront curvature effects.

Linear frequency (chirp) modulation.

Migration through range cells.

Filter (or patch) width for space-variant post-filter. 
$m_{\text {opt }}(c) \quad$ Optimal space-variant filter length $m$ (yielding lowest computational complexity), for a given filter overlap $c$.

$m_{r} \quad$ Filter (or patch) radius, measured from $\left(x_{0}^{\prime}, y_{0}^{\prime}\right)$, for space-variant post-filter.

$o_{x}^{\prime}, o_{y}^{\prime} \quad$ Oversample ratio in cross-range and range, respectively.

OSA Overlapped subaperture algorithm (for image formation).

$P_{\theta}(U) \quad$ Fourier transform of tomographic projection function $p_{\theta}(u)$.

PFA Polar-format algorithm.

PFA/SVPF Polar-formatting in conjunction with space-variant post-filtering.

$p_{0}^{\prime} \quad$ Properly placed Cartesian coordinate that is free of geometric distortion induced by wavefront curvature.

$p_{f}^{\prime} \quad$ Geometrically distorted position of a coordinate that would otherwise be placed at $p_{0}^{\prime}$ in the absence of wavefront curvature.

$\phi_{\epsilon} \quad$ Total Fourier domain phase error.

$\phi_{1} \quad$ Linear Fourier domain phase term.

$\phi_{1 \epsilon} \quad$ Linear Fourier domain phase error term.

$\dot{\phi}_{2 \epsilon} \quad$ Quadratic Fourier domain phase error term.

$\phi_{2 t} \quad$ Total quadratic Fourier domain phase error.

$\phi(\theta, k) \quad$ Complex phase of video signal at aperture angle $\theta$ and time scale factor $k$.

$\phi_{1 x_{0}^{\prime}, y_{0}^{\prime}}\left(X^{\prime}, Y^{\prime}\right) \quad$ Slant plane Fourier phase term with respect to the image domain position $\left(x_{0}^{\prime}, y_{0}^{\prime}\right)$ and frequency extent $\left(X^{\prime}, Y^{\prime}\right)$. 
$\dot{\phi}_{x_{0}^{\prime}, y_{0}^{\prime}}\left(X^{\prime}, Y^{\prime}\right) \quad$ Rate of change of quadratic phase error with respect to image domain coordinate $\left(x_{0}^{\prime}, y_{0}^{\prime}\right)$ and frequency extent $\left(X^{\prime}, Y^{\prime}\right)$.

$\Psi \quad$ Depression angle, from horizontal.

QPE Quadratic phase error.

RMA Range (seismic) migration image formation algorithm. Motion compensation to a line yielding a chirp in azimuth and increased squint-mode upsampling. Inherently compensates wavefront curvature.

$r_{0}$

$r_{c}$

$r_{t}$

$r_{x}$

$\rho$

$\rho_{u}$

$\rho_{x}^{\prime}, \rho_{y}^{\prime}$

SNR

SYNTARG

SVPF

$s_{x}^{\prime}, s_{y}^{\prime}$

$s_{p}\left(x^{\prime}, y^{\prime}\right)$
Slant plane standoff range at aperture center.

Slant plane distance at aperture center from platform to a point target at $p=(\rho, \gamma)$.

Slant plane distance from platform to a point target at $p=(\rho, \gamma)$.

Slant plane distance from platform to scene center.

General image resolution, as projected onto ground plane.

Image resolution in range, based on chirp bandwidth, $B_{c}$

Slant plane resolution in cross-range and range, respectively.

Signal-to-noise ratio.

Synthetic target generator (computer program).

Space-variant post-filter.

Slant plane scale factors (typically meters/pixel) in cross-range and range, respectively.

Contribution of point target $p$ to slant plane scene reflectivity function. 
TBP

TSA

$\tau_{c}$

$\theta_{L}$

$\theta_{s}$

UWB

VLSI

$W_{x}$

$X, X^{\prime}$

$X_{1}^{\prime}$

$x, x^{\prime}$

$Y, Y^{\prime}$
Time-bandwidth product (typically, $B_{c} \tau_{c}$ for LFM range chirp). Tiered subaperture algorithm (for image formation), a modified OSA.

Duration of LFM range chirp.

Angle at platform formed by edges between platform and scene center, and platform and scene edge $\left(x^{\prime} ; y^{\prime}\right)=(L, 0)$.

Squint angle, relative to range direction. $+\theta_{s}$ : squint forward; $-\theta_{s}$ : squint backward; $\theta_{s}=0$ : broadside.

Ultra-wideband (spotlight-mode $\mathrm{SAR}$ ), $f_{c} \approx 470 \mathrm{mHz}$, vulnerable to wavefront curvature affects. Often requires extended apertures to achieve sufficient cross-range resolution $p_{x}$.

Very large scale integration (as applied to OSA or TSA processing).

Along-track beam width (patch illumination) at the ground.

Cross-range spatial bandwidth (frequency extent) in ground plane and slant plane, respectively. Or, the cross-range axis in Fourier ground plane and slant plane, respectively.

Maximum cross-range spatial bandwidth (frequency extent) in the slant plane.

Cross-range distance in the image ground plane and slant plane, respectively. Or, cross-range axis in image ground plane or slant plane, respectively.

Range spatial bandwidth (frequency extent) in the ground plane and slant plane, respectively. Or, range axis in Fourier ground plane or slant plane, respectively. 
Maximum range spatial bandwidth (frequency extent) in slant plane.

$y, y^{\prime}$ Range distance in image ground plane and slant plane, respectively. Or, range axis in image ground plane or slant plane, respectively.

$z^{\prime} \quad$ Length of synthetic aperture. 


\section{Chapter 1}

\section{Introduction}

\subsection{Overview}

The classic approach to SAR image formation from phase history data collected in the spotlight-mode has been the polar-format algorithm (PFA). This algorithm was developed in 1974 by Dr. Jack Walker and served as the basis of his Ph.D. dissertation [1]. Later, this work was published in the IEEE Transactions on Aerospace and Electronic Systems journal [2] and patented in 1980 [3]. The polar-format algorithm is still popular today because of its straightforward implementation and robustness in constructing images of large scenes without introducing phase discontinuities. In contrast to the stripmap-mode SAR, which is a radar with a fixed look angle for a given collection (see Figure 1.1), the spotlight-mode SAR slews its antenna as the aperture is flown, thereby staying aimed at the scene center for the entire duration of the collection, as shown in Figure 1.2. Due to its extended dwell time on a given scene, the spotlight-mode SAR is capable of higher along-track resolutions and typically requires less transmit power than the strip-map SAR. The disadvantage of spotlight-mode SAR is that a smaller ground patch is imaged compared to strip-map SAR. However, it is possible (and in fact common) to mosaic spotlight-mode images into larger image patches. 


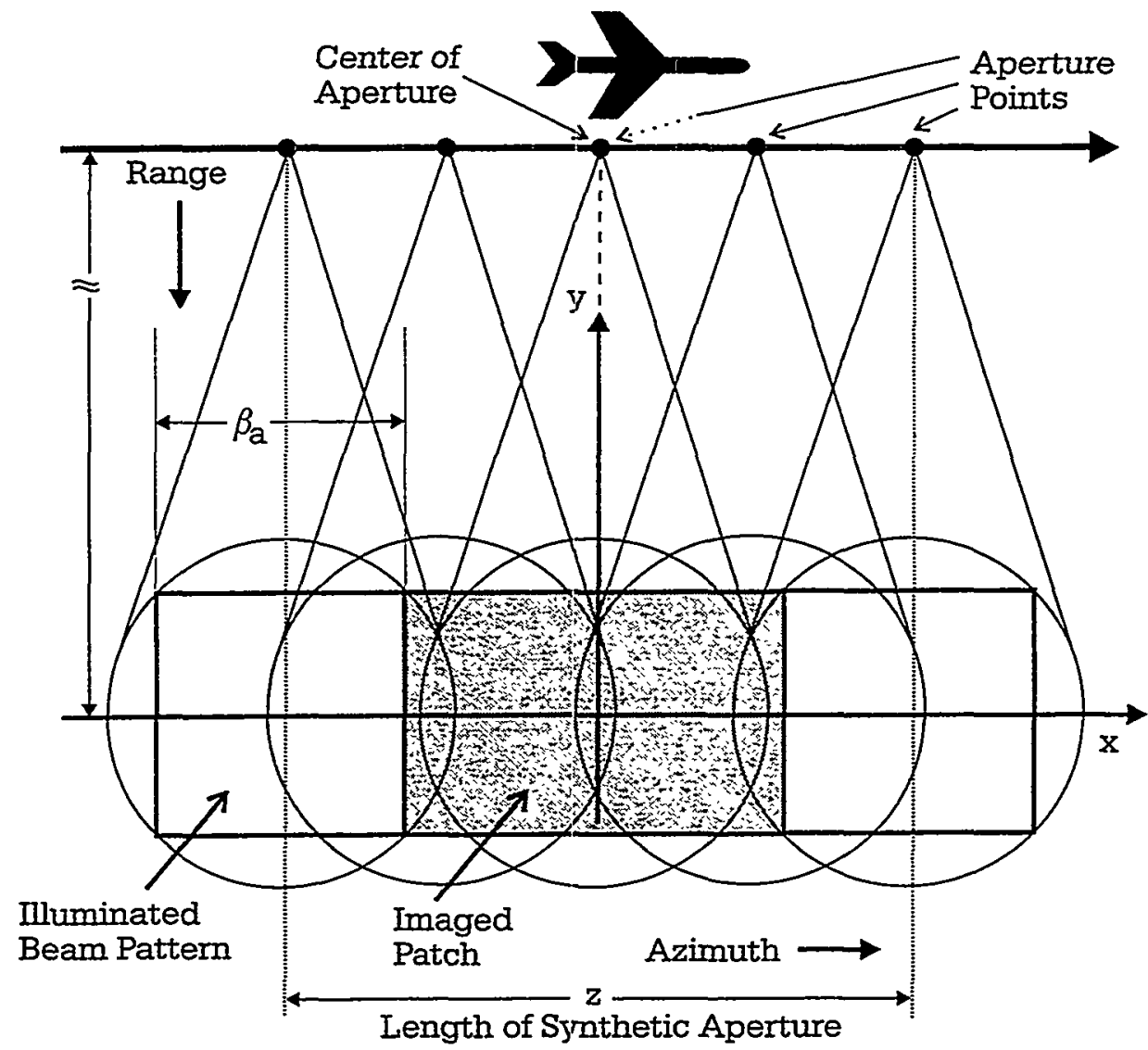

Figure 1.1: Stripmap-Mode SAR Imaging Geometry

In polar-formatting, the collected phase history data are described in terms of a slice of the three-dimensional Fourier transform of the scene reflectivity, obtained on a polar raster, as shown in Figure 1.3 (a). Known as the tomographic approach to $S A R$, the analogy between spotlight-mode SAR and tomography was first proposed by David C. Munson and his colleagues, and presented formally in 1983 [4]. It was then revisited and recast by Ausherman, et. al. [5]. Munson's method expounded on the preliminary work of Walker, yet failed to cover several important points. For instance, the imaging of three dimensional (elevated) targets was not discussed in Munson's paper. A complete three-dimensional tomographic model that was later developed by Jakowatz and Thompson accounts for range-dependent layover in the scene [6]. Jakowatz, et al, [7, pp. 355-365] in 1996 addressed the effects of certain 


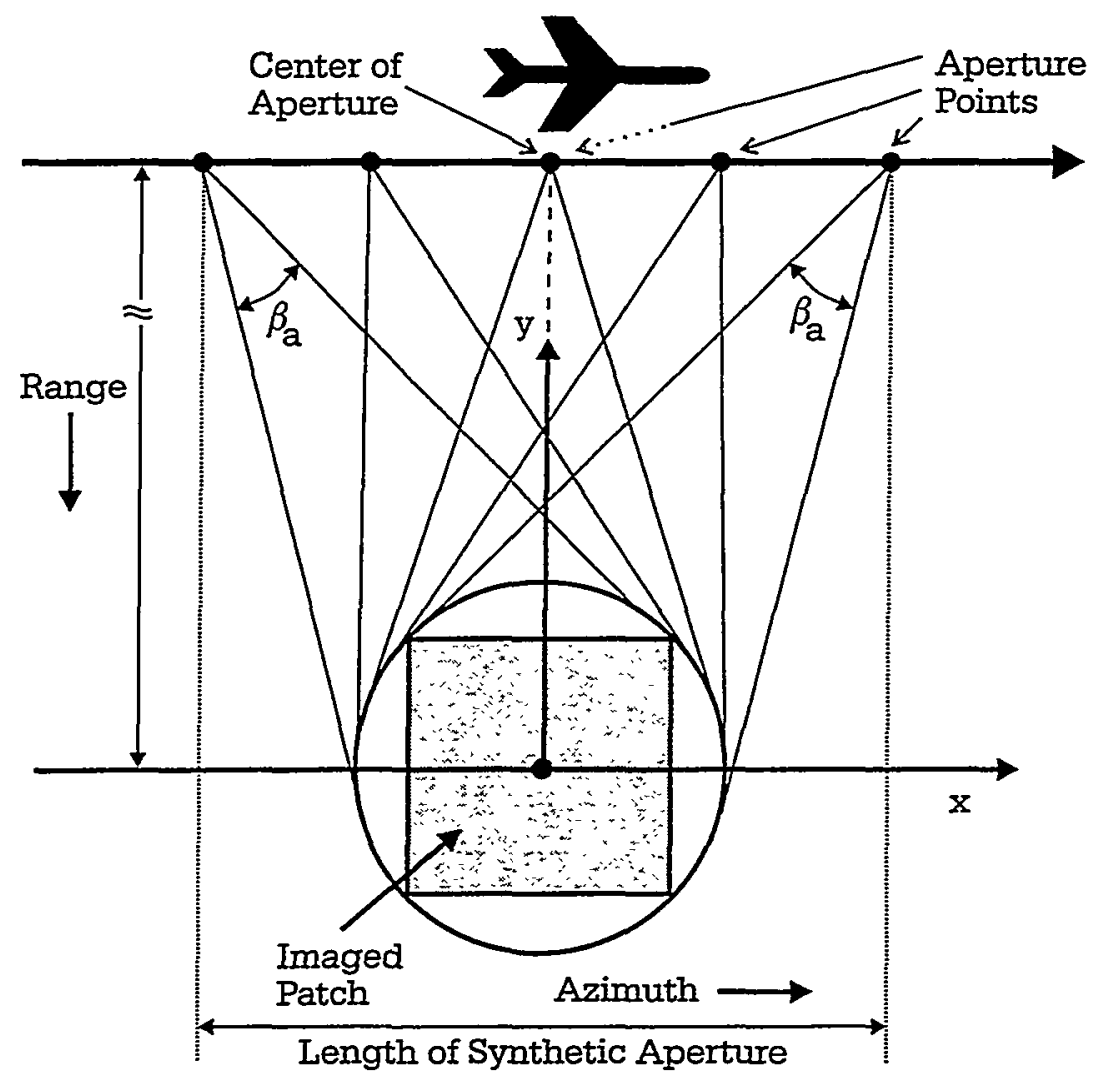

Figure 1.2: Spotlight-Mode SAR Imaging Geometry for Polar-Formatting

phase errors in polar-formatted imagery that arise from assumptions in the tomographic spotlight-mode SAR model. The first is deramp residual phase error, which is a residual of the signal deramp process used in the range compression of LFM (chirp) radar processing. This phase error induces a geometric distortion on the formed imagery (with the effect of distorting a square scene into a keystone) and also some mild defocus, yet is mitigated through a well-known procedure known as deskew processing. Deramp processing is discussed in detail in [7, pp. 396-398]. Deskew processing for mitigating deramp residual phase error is discussed in $[7, \mathrm{pp}$. 363$365]$, and alternative methods are presented in $[4,8,9]$. Typically, this phase error is negligible and can be ignored in nearly all SAR imaging scenarios. A second undesired effect is that of wavefront curvature, which is based on the faulty assumption of strictly planar wavefronts being transmitted by the radar. These are potentially 
more serious in terms of image degradation than the deramp residual phase errors, since wavefront curvature phase errors can induce a significant geometric distortion and a space-variant defocus of the imaged scene for some imaging geometries. These defocus effects are troublesome to negate due to their space-variant nature. This dissertation is based on a concept originally proposed by Jakowatz, et al, [10] in which a geometric model of the phase return from a point target is used to derive (and ultimately compensate) the phase errors. That concept was further expanded to include squint-mode imaging, as described by Doren, et al, in [11]. This dissertation provides a comprehensive treatment of the subject including wavefront curvature examples, a complete mathematical development of the phase error model, methods for efficient computer implementations, examples of post-filtered imagery and a timing analysis comparing PFA with wavefront curvature correction to other image formation methods. Furthermore, the detailed mathematical analysis presented here avoids several of the original simplifying assumptions which limited the utility of the original algorithm.

The traditional polar-format algorithm consists of a polar-to-rectangular interpolation of all complex-valued, phase history data points from a points from a spotlightmode collection (see Figure 1.3 (b) and (c)). This interpolation, in conjunction with motion compensation to the scene center, negates migration through range cells (MTRC), also known as range walk (see Figure 1.4). Then, an inverse Fourier transform of these data (as projected onto a chosen two-dimensional plane, based on Munson's model) forms the SAR image. Since the derivation of this technique relies upon the unrealistic assumption of strictly planar wavefronts in the transmitted microwave pulses, the polar-format algorithm cannot compensate these wavefront curvature effects. Consequently, any actual amount of curvature present in these wavefronts introduces the geometric distortion and space-variant defocus in the SAR image, as formed by the polar processor. In some literature, this wavefront curvature (and the associated distortion and defocus effects) is referred to as differential range curvature, or more simply (and loosely), range curvature. For clarity, this dissertation 
makes a semantic distinction between range curvature, which is the range-oriented movement of a target during the duration of the aperture (as shown in Figure 1.4 (b)), and wavefront curvature, which induces the distortion and defocus effects associated with the faulty assumption of planar wavefronts. The polar-format algorithm, until now, did not compensate wavefront curvature effects and was considered to be mostly but not completely range curvature compensating, since it did account for MTRC but not wavefront curvature. This dissertation deals strictly with negation of the wavefront curvature effects, and in conjunction with MTRC compensation; renders the polar-format algorithm as fully range curvature compensating.

In polar-formatted imagery, the geometric distortion induced by wavefront curvature, due to first-order (linear) phase errors, takes on the form of an annulus in the processed imagery, as shown in Figure 1.5. This distortion can be rectified in a straightforward manner by appropriate post-warping of the image. The space-variant defocus is based on second-order (quadratic) phase errors and is not removable via post-warping. The magnitude of this defocus effect is a function of the range and cross-range position of the target and becomes greater for those targets placed further in range and cross-range from the scene center. Furthermore, the space-variant defocus is more pronounced for collections taken with radars of low center frequency, high resolution, at close standoff ranges, or when imaging very large scenes. Figure 1.6 (a) simulates the effects of space-variant defocus due to wavefront curvature on a PFA processed scene of the US pentagon, while Figure 1.6 (b) has the defocus effects removed, as well as being geometrically rewarped.

Consider a typical L-Band $\left(f_{0}=1.25 \mathrm{GHz}\right)$ airborne spotlight-mode radar, imaging a $5 \mathrm{~km}$ diameter patch at range of $5 \mathrm{~km}$ and having a cross-range resolution of 0.6 $\mathrm{m}$. The quadratic phase error induced by wavefront curvature at the far range of the scene is sufficient to severely defocus this point in the scene, thereby rendering that part of the imagery as useless [12, pp. 437-439]. This is due to the impulse response (IPR) broadening that occurs because of the quadratic phase error, as analyzed in Appendix $A$ of this dissertation. This degradation is due to the lack of compensation 


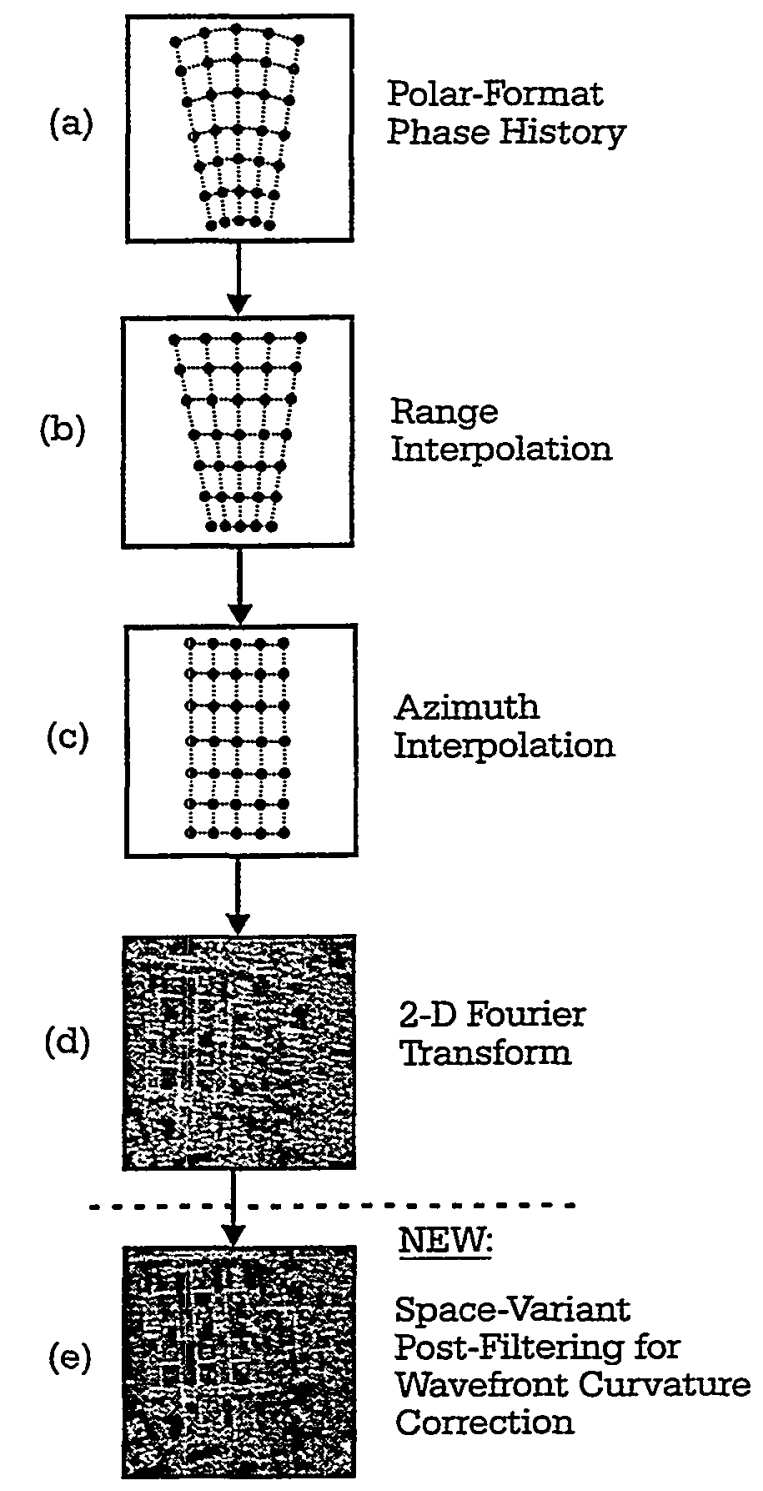

Figure 1.3: Basic Steps to the Polar-Format Processing Algorithm

for wavefront curvature in the polar-format algorithm under these conditions, and not because of limitations of the radar hardware itself. Clearly, it is not desirable for a SAR capable of high resolution to be limited by shortcomings in the image formation algorithm. Perhaps more tragic is that this limitation is not even a shortcoming of the image formation method, but instead the approximations of the mathematical model used to derive the polar-format algorithm. As SARs attain ever increasing resolutions, wavefront curvature effects become more prominent and the resulting 


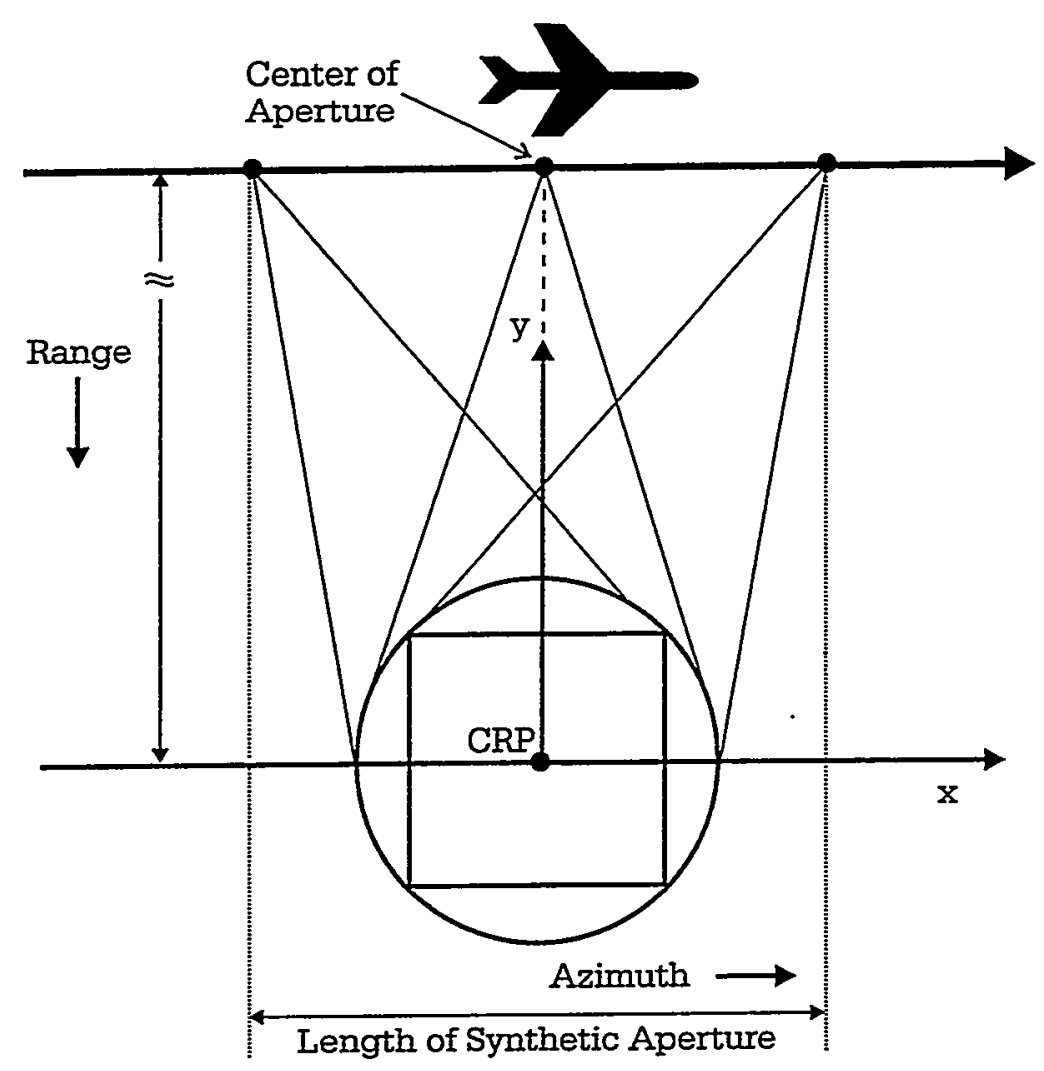

(a)

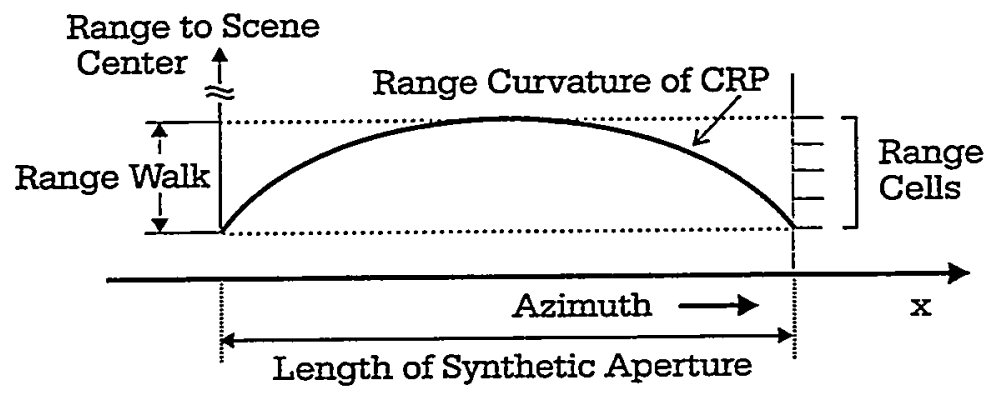

(b)

Figure 1.4: Range Curvature of CRP During Spotlight-Mode Collection

quadratic, space-variant defocus becomes a major limiting factor in the quality of polar-formatted imagery.

Prior to this dissertation research (and the initial research by Jakowatz on which it is based), the only "solution" to avoiding the wavefront curvature defocus effects 


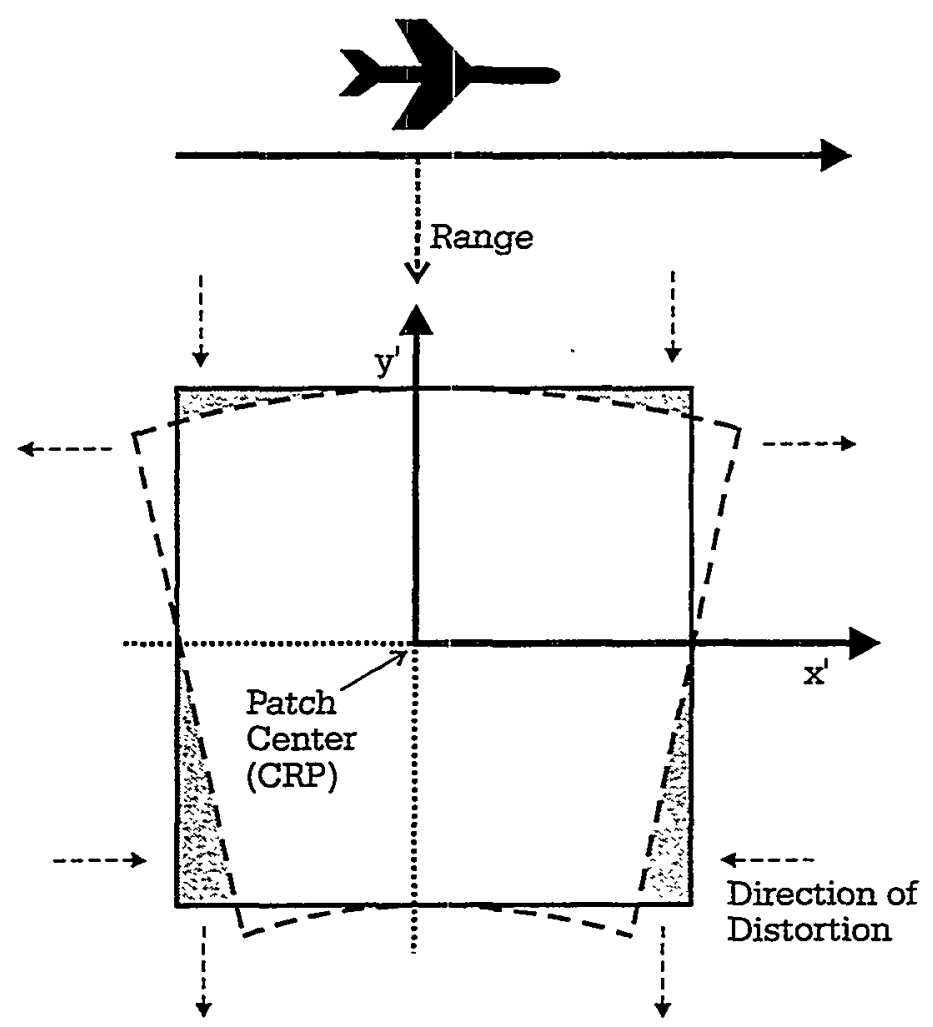

Figure 1.5: Geometric Image Distortion Induced by Wavefront Curvature

in polar-formatted SAR imagery has been to reduce the size (diameter) of the reconstructed scene relative to the standoff distance of the platform, or by reducing cross-range resolution by shortening the synthetic aperture length. A generalized polar-format processor, first proposed in 1991, serves to reduce the residual phase errors arising from wavefront curvature, thereby increasing the maximum possible patch size [13]. However, the generalized polar-format processing approach, while computationally efficient, still requires restrictions in patch size (though somewhat relaxed compared to traditional PFA) in order to avoid visible defocus effects in the formed imagery. This dissertation research "breaks ground" in that it compensates the effects of quadratic defocus induced by wavefront curvature in polar-formatted spotlight imagery, with little increase in computational cost and without imposing arbitrary constraints on scene size, squint, resolution or standoff range. Furthermore, this compensation can be achieved to within sub-pixel accuracy by carefully 


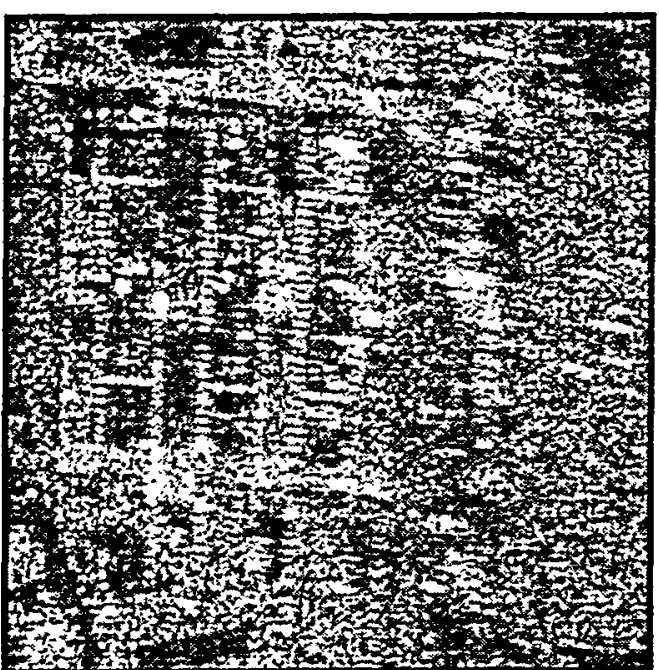

Before Removal

(a)

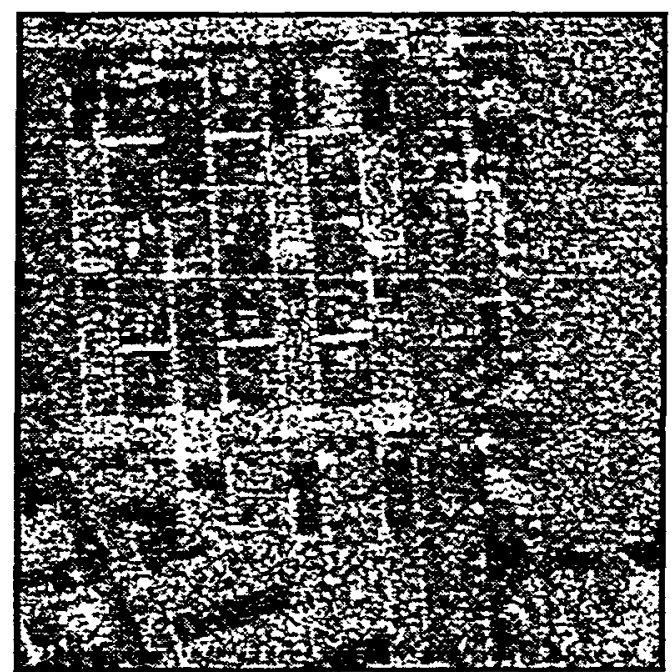

After Removal

(b)

Figure 1.6: Simulated Wavefront Curvature Defocus Effects Before and After Removal

controlling the residual quadratic phase errors, regardless of scene size or resolution, ensuring there is no visible degradation of the formed imagery due to these errors. Chapter 2 introduces the wavefront curvature problem and Chapter 3 motivates the space-variant post-filtering solution for polar-formatted spotlight-mode imagery.

\subsection{Introducing the Space-Variant Post-Filter}

This research has culminated in a method for minimizing the quadratic defocus effects associated with wavefront curvature in polar-formatted spotlight-mode SAR imagery for all squint angles. This method incorporates a space-variant, image-domain filter which refocuses the formed, complex-valued imáge. The filtering is performed as a post-processing step to the polar-format algorithm, and thereby preserves all the advantages of polar-format processing including its simplicity, autofocus capabilities, and effectiveness in forming large images with no subpatch processing. It will be shown that the quadratic defocus effect of wavefront curvature on a spotlight-mode 
SAR image formed with polar-formatting is a space-varying one which occurs in the cross-range (azimuth) direction only. Thus, a one-dimensional, space-variant filter is sufficient for compensating the defocus. This has the advantage of computational simplicity, as well as being amenable to parallel processing environments. Furthermore, it will be demonstrated that the computational burden involved in performing the space-variant restoration to remove the wavefront curvature defocusing effects is not particularly severe. For a certain (significantly large) set of imaging scenarios, it will be shown to be as small as thirty percent of the polar-format image formation time.

The image-domain, space-variant post-filter (SVPF) is based upon an analytical derivation of the phase error that is induced by curved wavefronts, as presented in Chapter 4. This is accomplished via a geometric model of the imaged scene with respect to the radar's flight path, and subsequently deriving an equation for the phase of the return signal for a given point, $(x, y)$, in the imaged scene. Next, a two dimensional Taylor series expansion is applied to the phase equation, allowing the phase return signal to be represented by its polynomial components. The linear, first-order terms of this series represent the position of the target's radar return in the imaged scene, and is consistent with Munson's tomographic paradigm. However, included are linear distortion terms that result in a space-variant, geometric warping of the imaged scene, ignored by Munson. This distortion is normally (and has been traditionally) removed in a post-processing warping procedure. The second-order terms, due to the quadratic phase error (QPE) induced by wavefront curvature, are much more serious in terms of image degradation and account for the space-variant defocus of targets in the imaged scene. This defocus effect (and its compensation) serves as the basis of this dissertation. Higher order phase error terms also exist, but are typically small in magnitude and are ignored since they have little defocusing effect on the imaged scene, at least given the state of SAR technology today. The space-variant post-filtering method could be easily extended to deal with higher order phase errors, if this becomes necessary due to future advances in SAR hardware 
technology that greatly improve upon current system resolutions or spotlight patch sizes.

Typically, the space-variant post-filter operates on the formed, complex imagery in the frequency domain, although convolution in the spatial domain is also possible, and is mathematically (though not computationally) equivalent. In the frequency domain, the algorithm works by calculating the one-dimensional, quadratic phase error function for different points along the scene, and multiplying this function by the complex conjugate of the Fourier domain data at that point in the scene. The filter function varies as does the $(x, y)$ location of the scene where it is being applied. The procedure is made computationally efficient by varying the filter function only as rapidly as required to maintain the residual blur at an acceptable level. Furthermore, the filter function perfectly compensates the quadratic blur only at $(x, y)$, yet serves as a reasonable approximation to the phase error for some region of points around $(x, y)$. Therefore, by carefully choosing the length of the filter, and also its spacing in $(x, y)$ according to certain criteria as dictated by the imaging geometry and radar frequency parameters, computational efficiency can be maximized while maintaining residual blur at an acceptable level, as described in Chapter 5 .

\subsection{Alternative Spotlight-Mode Image Formation Methods}

The space-variant post-filter for polar-formatted spotlight-mode SAR imagery is general in that it corrects for wavefront curvature in broadside as well as squinted data collections, with no significant computational penalty for correcting squint-mode images. Other "fashionable" spotlight-mode SAR algorithms, such as the range migration technique (also known as seismic migration, or RMA), and recent enhancements such as frequency domain replication (FReD), have been developed to accommodate these wavefront curvature effects. The range migration algorithm has its roots in 
the field of seismic signal processing for imaging the Earth's substrata, as described in [14] and [15], and was originally applied only to stripmap SAR collections. Subsequent papers by Prati, et al, demonstrate the extension of RMA as it applies to spotlight-mode processing $[16,17]$, and it was thought to be the logical successor to polar-format processing due to its inherent ability to compensate for wavefront curvature. However, the along-track upsampling of the phase history data required of the original version of RMA can, in certain instances (spotlight-mode), represent a major computational burden.

The FReD algorithm obviates the need to upsample, and is accordingly more efficient. It takes advantage of certain characteristics specific to polar-formatted $\mathrm{SAR}$ data. As presented in [18], FReD is based on the fact that when a discrete aliased spectrum is replicated a sufficient number of times, the resultant spectrum will contain the desired signal spectrum. Hence, the use of FReD allows the acquisition of data at normal spotlight-mode rates and obviates the need for FFTs larger than those required for normal spotlight-mode processing. However, this dissertation demonstrates that neither RMA nor FReD exhibit a clear computational advantage over space-variant post-filtering in conjunction with the traditional polar-format algorithm. Also, it is unknown how efficiently the RMA or FReD methods can be applied to non-straight-line flight paths [19], or how easily autofocus algorithms can be integrated into the image formation process. Furthermore, these algorithms are more sensitive to deramp residual phase errors than the polar-format algorithm. An efficient approach for applying the polar-format algorithm to non-straight-line paths is discussed in [20].

The chirp scaling algorithm (CSA) is a simplification of the RMA algorithm in that the nonlinear Stolt interpolation step (which compensates the range curvature of all scatterers) is replaced by a linear approximation. Thus, the CSA implementation requires only FFTs and complex vector multiplies, without any interpolations being necessary. It is more computationally efficient than RMA, but at a cost. Since this algorithm implements only the shift and linear components of the Stolt mapping, 
this approach becomes inadequate with the increase in resolution or scene size, and residual phase errors leading to image defocus become significant in these scenarios. Thus, the CS algorithm introduces some the same defocus effects that are removed via post-filtering of polar-formatted imagery formed with PFA. The enhancement of the CS algorithm is a current research topic that will not be discussed in detail in this dissertation. See $[21,22,23]$ for descriptions of the chirp scaling algorithm, and $[24,25,26,27]$ for discussions of some ongoing research regarding the CSA. Additionally, an excellent comprehensive treatment of the CS algorithm is found in the dissertation of G. Davidson [28], and a massively parallel implementation of the CSA is discussed in [29].

Yet another image formation method, convolution back-projection (CBP), has been suitably modified to compensate for wavefront curvature effects. This method is discussed in two papers by Jerald Bauck [30,31], which served as the preliminary work leading to his Ph.D. dissertation [32]. In discussing the wavefront curvaturecorrecting variation of CBP, Bauck states that with his method, "The task can be fairly easy, although requiring somewhat more computation that Fourier-based methods which use an FFT but do not correct for wavefront curvature." In this dissertation, it will be shown that PFA, the most basic and established Fourier-based method, even with the additional burden of space-variant post-filtering, is still significantly better than CBP in terms of overall computational burden, even in the simpler case when CBP has not been modified to correct for wavefront curvature. Furthermore, CBP is not considered a computationally efficient algorithm to implement on a parallel computer, as it typically requires the programmer to make a tradeoff between excessive memory requirements and significant communications overhead on distributed-memory computers [33]. Additionally, the application of autofocus algorithms for uncompensated platform motion is difficult when using CBP. This is due to the lack of an intermediate, Cartesian-coordinate, range compressed grid on which to apply autofocus algorithms. This pitfall also applies to algorithms based on the modified chirp $z$ transform (MCZT), which are variations of the chirp 
scaling algorithm $[34,35]$. Unlike the CBP and MCZT algorithms, polar-formatting has been efficiently ported to a parallel processing environment. This topic has been well researched and covers software implementations on parallel computing platforms $[36,37,38]$, as well as direct silicon implementations in VLSI [39]. In fact, even the phase-gradient autofocus algorithm, which compensates for unknown platform motion error, has been successfully ported to the parallel processing environment [40]. In similar fashion, the new SVPF algorithm is also amenable to parallel processing, as is discussed in Chapter 7, providing the opportunity for a complete, high performance polar-format image formation algorithm which includes both autofocus and post-filtering for wavefront curvature correction.

Other spotlight-mode image formation methods are used within the SAR community, but are less prevalent. For example, the Twin-Otter SAR Testbed at Sandia National Laboratories, capable of operation on four frequency bands, features realtime image formation at fine resolutions in both stripmap and spotlight modes using the overlapped subaperture (OSA) algorithm [41]. Originally designed to take advantage of customized VLSI hardware, OSA was one of the first algorithms to form SAR images in real-time, using computing equipment on-board the radar platform $[42,43]$. This image formation algorithm was patented in 1997 by Bryan Burns and Tom Cordaro of Sandia National Laboratories [44]. While utilized in a number of radar systems, recent research indicates OSA and its variants such as the tiered subaperture algorithm (TSA) $[45,46,47]$ hold no clear advantage in throughput over multiple-processor versions of PFA [48]. Furthermore, while OSA does inherently correct for wavefront curvature, it may sometimes have problems correcting higher-frequency, uncompensated platform motions when large subapertures are implemented.

A comparison of several different spotlight-mode image formation methods, in terms of computational demands, is presented in Chapter 6 . The reader is encouraged to read the cited reference materials for a full mathematical understanding of these algorithms, and to refer to Chapter 6 for the run-time analysis and comparison 
of these methods. Specific implementations of image formation algorithms do not serve as the final word in the computational efficiency or the superiority of one algorithm to another. However, the comparisons do give a general, ballpark-sense of the competitiveness of each algorithm in terms of computational burden.

\subsection{Dissertation Objectives}

The traditional polar-format algorithm, PFA, is a robust, efficient, and well proven method for spotlight-mode SAR image formation. However, the derivation of this technique relies on the unrealistic assumption of strictly planar wavefronts in the transmitted pulses. As the system resolution increases, or large scenes are imaged at close range or low center frequencies, the amount of wavefront curvature actually present differs significantly from the planar wavefront approximation. Consequently, for these collections, a significant amount of geometric distortion and space-variant defocus is present. While the geometric distortion is easily removed via a postwarping procedure, the second-order phase terms leading to space-variant image defocus are more problematic.

Dating back to Walker's pivotal paper in 1980, Range-Doppler Imaging of Rotating Objects [2], he presents a method for separating the first and second-order quadratic phase error terms using a Taylor series expansion, as is presented and fully developed in this dissertation. However, Walker does so only to quantify the scene size limitations of polar-formatted SAR data, without attempting to propose a solution to the wavefront curvature problem, as is done in this dissertation. Instead, he states that the coefficients associated with the second-order phase error terms depend on both object point location and on processing aperture location, and that, "In principle, these error terms can be removed by refocusing the data processor... (but) this approach is generally difficult to implement." Consequently, prior to this dissertation and the preliminary papers by the author and his associates $[10,11]$, 
space-variant defocus due to wavefront curvature was not compensated but simply reduced to an acceptable level by putting upper limits on the imaged scene size when using the polar-format algorithm, as suggested by Walker. This was an unreasonably restrictive constraint that limited the versatility of polar-formatting when imaging large scenes. Indeed ironic, given that otherwise, PFA lends itself nicely to imaging large scenes, due to its simplicity, in the absence of wavefront curvature. While it is true that second-order phase error terms depend on both object point location and processing aperture location, this dissertation proposes, derives, and demonstrates methods for space-variant post-filtering that greatly reduce the computational processing burden, and they are not difficult to implement. This is true even for squinted collections, whereby the radar is not looking at the imaged scene from a right angle, but instead is slewed forward or backward, as shown in Figure 1.7.

The primary contribution of this dissertation is the development of the squintmode geometric model, which expands upon the restricted, broadside model proposed by Jakowatz in [7, pp. 355-365], and demonstrated in [10]. This extension generalizes the space-variant post-filter and extends its utility to many practical imaging scenarios, which typically are not restricted to broadside. Furthermore, this dissertation focuses upon computer implementation issues which relate to computational complexity of the filter. The goal is to achieve the highest possible efficiency, while still maintaining an acceptable quality of refocus. While not providing exact compensation for the second-order phase errors, the residual blur is constrained to sub-pixel levels while maintaining a very acceptable computational burden. The empirical running times of SVPF in squint and broadside modes are described in Chapter 6 .

\subsection{Summary}

This dissertation covers the motivation, theoretical development, implementation and analysis of the new space-variant post-filter in conjunction with the traditional 


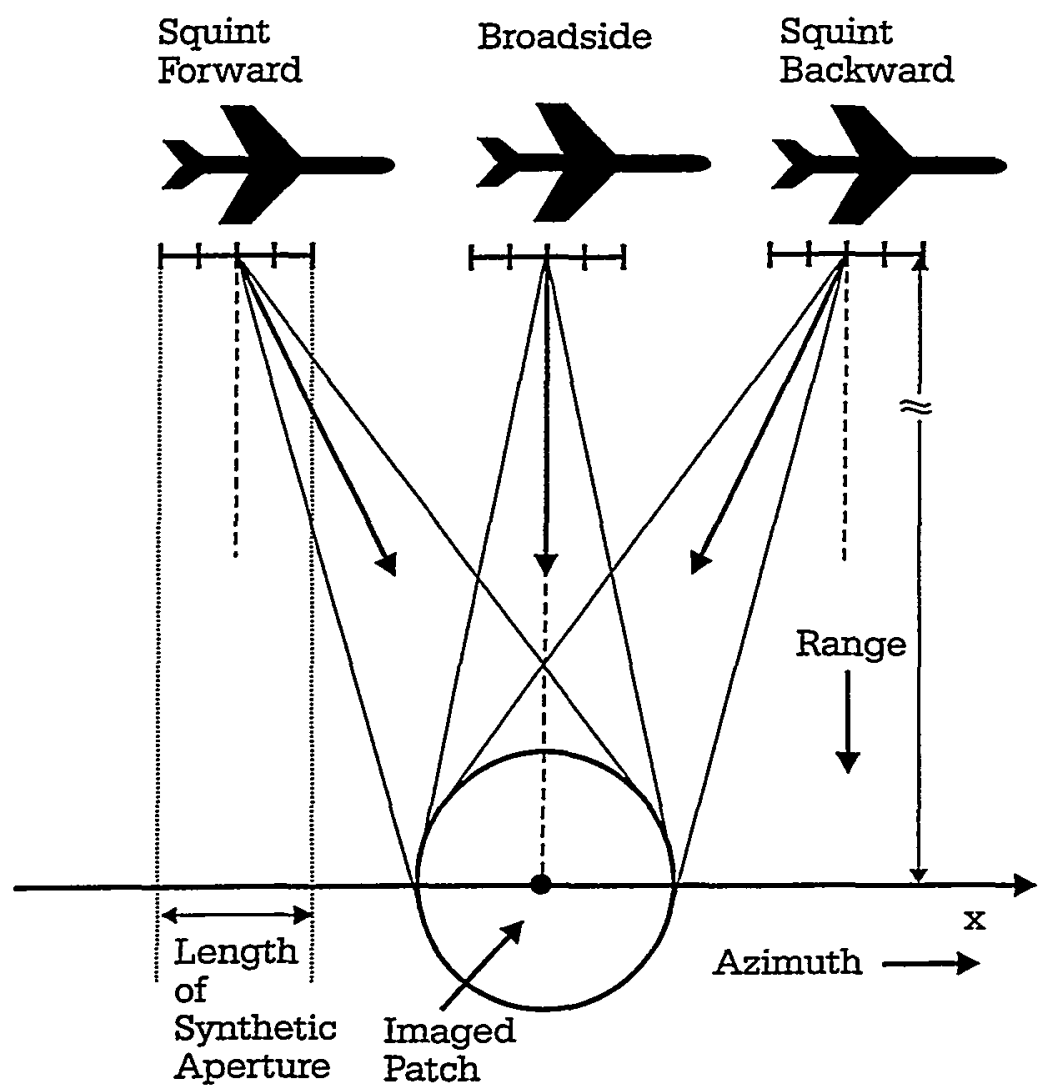

Figure 1.7: Squint Modes for Spotlight-Mode SAR Collections

polar-format algorithm. Its primary contributions are the development of the generalized squint-mode model, and the focus upon computational issues which render the filter efficient and practical in real imaging scenarios. This post-filter serves to compensate the space-variant defocus effects of wavefront curvature in polarformatted data, which are due to the faulty assumption of planar wavefronts being transmitted by the radar. The filter is precalculated from the theoretical derivation of the wavefront curvature effect. It is shown that the defocus effects are based on second-order phase errors in the cross-range direction only, so that a simple, efficient one-dimensional filter is sufficient for refocus. The implementation of this filter is discussed, including filter length and overlap issues which effect both computational burden and restorative quality. Examples of its capabilities and limitations are provided and guidelines are offered for maximizing its computational efficiency. 
Tradeoffs between this method and other spotlight-mode image formation techniques are discussed with regard to computational burden, image quality, and imaging geometry constraints.

This research concludes that space-variant post-filtering, in conjunction with $\mathrm{PFA}$, provides an effective spotlight-mode image formation solution that solves the wavefront curvature problem for all standoff distances and patch sizes regardless of squint, resolution or radar frequency, while constraining residual defocus to subpixel levels. Additional advantages are that SVPF is not iterative and has no dependence on the visual contents of the scene. Thus, it has a deterministic computational complexity which typically adds as little as thirty percent to the overall image formation time. Furthermore, no other image formation technique has been shown to have a clear computational advantage over polar-formatting with space-variant post-filtering. Consequently, polar-formatting in conjunction with SVPF should be considered as a viable candidate for a spotlight-mode image formation processor when wavefront curvature effects are present.

\subsection{Organization of this Dissertation}

This dissertation is a combination of background information, illustrative examples, mathematical theory, and a computer-based demonstration of the results. First, this dissertation introduces the necessary background information which forms the basis of the space-variant post-filtering algorithm for polar-formatted SAR imagery. Subsequently, it includes a theoretical development of space-variant post-filtering, and for comparative purposes, illustrative examples of wavefront curvature effects in uncompensated polar-formatted spotlight-mode imagery as well as the corrected images processed using the space-variant post-filtering algorithm. Empirical timing tests are provided for the new SVPF algorithm in conjunction with PFA, as well as those for other popular methods of image formation. Conclusions include the per- 
formance analysis of the space-variant post-filtering method, with a comparison to some of the well known, competitive techniques for spotlight-mode image formation. Pitfalls of the approach are discussed as well as suggestions for further work. An appendix is included which develops the mathematics for the IPR broadening that occurs in the presence of quadratic phase errors. Every effort has been made to ensure consistency with regard to terminology, variable names, and labels and axes within diagrams. Acronyms and variable names are described in the glossary.

The dissertation is organized as follows:

- Chapter1 - Chapter one serves as a general introduction to space-variant post-filtering for wavefront curvature correction in spotlight-mode SAR imagery. The dissertation is summarized and a brief historical perspective is presented. The motivation for this research is given as well as a synopsis of the significant contributions. The organization of the dissertation is discussed and a brief research summary is presented.

- Chapter 2 - This chapter presents an overview of spotlight vs. stripmap imaging and presents spotlight-mode SAR in terms of a tomographic process. The mathematical relationship between SAR and tomography is described in terms of the projection-slice theorem. A description of the polar-format algorithm is given, and assumptions and omissions leading to the wavefront curvature effects are discussed, based on the tomographic framework presented.

- Chapter 3-A geometric model is presented which serves to explain the wavefront curvature phenomenon. Illustrations demonstrate the effects of wavefront curvature distortion and defocus on real SAR imagery. The space-variant behaviors are discussed in terms of how they distort and defocus the formed imagery. Scene size restrictions are given for traditional polar-formatted imagery, which does not compensate wavefront curvature. The concept of a spacevariant post-filter for wavefront curvature correction is introduced. 
- Chapter 4-Chapter four presents the geometric models by which the spacevariant filter is designed. These models are based on the contribution of a point target to the overall scene reflectivity function in terms of phase returns. Using these models, the filter parameters are mathematically derived in terms of first, second and higher order phase error terms via a Taylor series decomposition. Linear phase error terms in the Taylor series represent geometric distortion, while quadratic terms induce space-variant defocus. The concept of synthetic target generation is introduced, which is based on the same geometric models.

- Chapter 5 - This chapter presents a filtering method for the compensation of the quadratic phase errors quantified in Chapter 4. The concept of spacevariant post-filtering for refocus is instroduced, and the need for space-variant adjustments is justified. Illustrations show how the filter is applied to the spatial-domain complex image data, with regard to filter length and overlap, and how efficiency is improved by varying the filter only as often as necessary. The geometric distortion of the image is taken into account, though this is not compensated until after refocus.

- Chapter 6 - In Chapter six, the computational complexity of space-variant post-filtering is discussed in terms of complex-number multiplies and asymptotic compelxity. Computational burden versus the quality of refocus is discussed, Also, other "fashionable" methods of image formation are introduced which compensate for wavefront curvature, including range migration (RMA), frequency domain replication (FReD), overlapped sub-apertures (OSA), and modified convolution back-projection (CBP). These methods are compared and contrasted in terms of complex-number operations, with regard to computing burden and the also the ability to compensate wavefront curvature effects. Run-time tests are performed on the various algorithms and the empirical performance results are presented.

- Chapter 7 - This is the conclusion chapter. It describes the pitfalls and short- 
comings of space-variant post-filtering. Topics for future work are suggested, the pursuit of which will extend the utility and performance of the method. Possible computational improvements are mentioned, including adaptive filter sizing and computational parallelism. A summary is given regarding significant contributions of the this work. Conclusions are drawn regarding the value of space-variant post-filtering to the SAR community.

- Appendix A - Derives the IPR broadening factor, which is a function of residual quadratic phase errors present in the imagery. This derivation is presented from the Fourier (frequency) perspective as well as the spatially-based (pixel spread) perspective. This mathematical derivation helps relate quadratic phase errors to the associated degradation in image resolution. The two techniques take a different approach but derive the same result. 
Chapter 1. Introduction

This page intentionally left blank 


\section{Chapter 2}

\section{Aperture Synthesis, Tomography and Polar-Formatting}

The synthesis of an along-track aperture, as a superposition of scene views along the flight path, provides for cross-range resolutions similar to those achievable in the range direction via chirped, wideband signals. Furthermore, for a synthetic aperture radar as opposed to a real aperture radar, the dependence of resolution on radar wavelength or standoff range is eliminated. If the radar beam is steered, or slewed, such that it maintains aim on the imaged scene, this spotlight-mode radar has the additional advantages of lower transmitted power for a given SNR, lower sampling rates and higher achievable resolution. When this spotlight-mode data is sampled on a polar raster, without regard to the instantaneous movement of the platform or targets, the tomographic paradigm can be used to describe the cross-range resolution capabilities of the SAR. With a direct analogy existing between computed axial tomography (CAT) and spotlight-mode SAR and their mathematical ties via the projection-slice theorem, spotlight-mode SAR can be cast as a tomographic process and described with the support of a simple signal processing framework. However, the tomographic paradigm is not without its assumptions or shortcomings, and these are necessarily (and unfortunately) cast into the spotlight-mode tomographic SAR 
framework. In particular, the assumption of planar wavefronts illuminating the scene, and the associated distortion and space-variant defocus that appear in polar-format processed imagery when this assumption is rendered invalid. This chapter serves as a brief introduction to synthetic aperture radar and presents the spotlight-mode SAR within the tomographic framework. The collected Fourier-domain phase history is described as a two-dimensional projection of the three-dimensional scene being imaged. The polar-format algorithm is introduced as a robust, yet simple method for Fourier inversion of the phase history into a image domain representation of the scene. The difference between the slant plane and ground plane images is described, as is their relationship to one another. The need for the computationally burdensome $2 \mathrm{D}$ interpolation is justified, with some reassurance given that this method is still competitive with other popular image formation methods, as will be shown in Chapter 6.

\subsection{The Range Resolution Problem}

The goal of a radar system is to distinguish, in both range and cross-range (azimuth), objects residing within the ground scene being illuminated. A number of range resolving techniques have been implemented over the years to achieve the desired range resolution; namely, continuous wave (CW) processing, and more recently, techniques incorporating the deramp processing and compression of linear frequency modulated (LFM) or "chirp" signals. These "complicated" (stretch) signals provide for wideband illumination of the target, allowing for finer resolutions than those achievable from the short-duration CW pulses. Furthermore, acceptable signal-to-noise ratios are maintained when using LFM systems because of their longer dwell times and consequently stronger radar returns. On the other hand, the range resolution of the simpler CW pulse is limited by the duration of the pulse, which must be quite short in order to avoid range-aliasing of the targets. The CW pulse is processed by demodulating the carrier with in-phase and quadrature sinusoids, followed by low-pass 
filtering. The Fourier analysis of the CW echo-ranging processor demonstrates that the radar transduces a narrowband estimate of the reflectivity function, where the range resolution depends strictly on radar bandwidth and not on center frequency or standoff range. The CW range resolution is given by

$$
\rho_{u}=\frac{c}{2 B_{e}}
$$

where $B_{e}$ is inversely proportional to the CW pulse envelope duration, $\tau_{e}$. That is, the time-bandwidth product of the CW pulse is unity. Consequently, with CW pulse transmission, any hope for higher resolution is lost when the pulse width is necessarily reduced, because the SNR of the reflected pulse is reduced as a consequence.

Using the FM chirp, a dispersed waveform, greater transmitted energy per pulse is possible compared to $\mathrm{CW}$, and signal-to-noise ratios increase. An FM chirp pulse is dispersed in time by a factor equal to its large time-bandwidth product, $B_{c} \tau_{c}$, compared to a CW burst pulse, with its time-bandwidth product of one. While the notion of the unity time-bandwidth product seems to have been violated, the LFM is not a simple pulse-like waveform, but instead is frequency modulated. As such, its bandwidth is not simply limited by the bandwidth of the envelope. The bandwidth of the FM chirp is given by

$$
B_{c}=\frac{\alpha \tau_{c}}{\pi}
$$

where $\alpha$ is the chirp rate in radian $^{2} \sec ^{-2}$ and $\tau_{c}$ is the chirp duration. Chirp signal (deramp) processing consists of the demodulation of the returned signal with in-phase and quadrature versions of the FM chirp, delayed appropriately by the round-trip time to the patch center, followed by low-pass filtering and range compression (Fourier processing). This effectively deconvolves the chirp waveform from the return signal, leaving an estimate of the terrain reflectivity function. The amount of pulse compression is given by $B_{c} \tau_{c}$, the time-bandwidth product of the waveform (in cycles). The utility of the FM chirp waveform in imaging radars is that its signal duration can be increased relative to that of the CW burst while maintaining the 
same effective bandwidth. In essence, the range resolving capability of the LFM chirp rests in its very large time-bandwidth product.

\subsection{Cross-Range Resolution Via Synthetic Aper- ture}

The previous discussion regards range resolution, yet cross-range (azimuth) resolution must also be addressed. Unfortunately, the solution to the range resolution problem via the notion of echo separation using high-bandwidth pulses seems irrelevant to the separability of targets in cross-range. However, the notion of interpreting the deramp-processed LFM chirp returns as direct transductions of certain spatial frequencies of the terrain reflectivity function is indeed applicable to cross-range resolution. This approach, known as the tomographic paradigm, has its roots in the field of medical tomographic imaging. The relationship between tomography and SAR will be detailed in Section 2.3. Unlike other cross-range resolution methods such as range-Doppler imaging $[2,5,49,50,51, \mathrm{pp} .33-83]$, which analyze the movement of the targets during aperture synthesis, the key concept of tomography is that a set of $X^{\prime}$ (cross-range) spatial frequencies are induced as a consequence of integrating differing views of the scene from the radar platform. Thus, cross-range resolution can be obtained by the coherent integration of a large number of pulses transmitted and received by the radar as it flies along a path, which becomes its synthetic aperture. As opposed to the range-Doppler paradigm for cross-range resolution, the continuous motion of the radar platform is not required to generate the $X^{\prime}$ spatial frequency data.

The detailed derivation and development of the tomographic paradigm has been thoroughly studied, and as such, is not the focus of this dissertation. However, enough background information on the tomographic paradigm will be presented here so that the concepts presented in this dissertation are properly motivated. For in- 
stance, given that the tomographic paradigm can be used to describe the generation of cross-range $X^{\prime}$ spatial frequency data via aperture synthesis, exactly why is this process necessary, and what are the real advantages to aperture synthesis, known as synthetic aperture radar? The object of incorporating a synthetic aperture is to increase the effective length (diameter) of the radar antenna, thereby increasing the scene resolution in the cross-range direction, independent of radar center frequency or standoff range. Prior to synthetic aperture radar (SAR), the azimuth resolution of the real radar system was the actual cross-range width of the beam, $W_{x}$, which was a function of the radar wavelength $\lambda$, the standoff range $R$ from the ground location to the radar platform, and the physical diameter of the radar antenna, $D$, such that

$$
W_{x}=R \frac{\lambda}{D}
$$

and

$$
\rho_{W}=W_{x}
$$

where $\rho_{W}$ is the real-aperture cross-range resolution. For certain high resolution imaging scenarios, this could require a real antenna length of thousands of meters, which would be foolishly impractical. On the other hand, given the ability to synthesize an aperture, a 10 meter antenna mounted to the belly of an aircraft could achieve the same cross-range resolution in these scenarios. Apparently, the only way to improve cross-range resolution for the real aperture system is to either reduce the platform standoff range or to reduce the angular beamwidth by reducing the wavelength, given that there are tight constraints (specifically, upper limits) on antenna diameter. However, because some radar wavelengths should be avoided in order to eliminate atmospheric effects and because certain radar platform types are constrained in standoff range, there are severe limitations to the practicality of real aperture radar. If finer resolutions and/or longer operating ranges are desired, an alternative to the real-aperture systems must be used, such as synthetic aperture radar. 


\subsubsection{Synthetic Aperture Radar in Stripmap-Mode}

Stripmap SAR is perhaps the oldest and most common type of SAR, and is still found in a variety of modern radar systems. With the radar being flown (approximately) in a straight line, a series of pulses are transmitted at intervals along the aperture in the fast time (range) direction, and range processing of the wideband signal is performed using LFM dechirp or another suitable method. As the aperture is flown, the direction of the radar antenna remains fixed, either at a right angle (broadside) to the scene being imaged or squinted forward or backward. Range pulses are collected along the aperture and processed in real-time or stored for future processing. Depending on the capabilities of the real-time processor and the capacity of the storage media, a strip of (theoretically) unlimited length may be imaged. The range-oriented motion of point scatterers during the time of radar illumination is then considered when resolving these targets in cross-range. Thus, for a given radar antenna platform position, the sequence of returns obtained along the flight path centered at this position is collectively processed to form the effective signal from an antenna much larger than is actually illuminating the ground. In this way, the aperture synthesis can be used to achieve fine cross-range resolution by the coherent integration of a series of radar returns transmitted at a number of positions along a flight path, thereby synthesizing the effects of a large physical antenna via data processing.

For a given point in the imaged scene, the longer this point is illuminated during the synthetic aperture collection, the longer the length of space (segment) over which the cross-range integration occurs. This effectively narrows the synthesized beamwidth, thereby increasing cross-range resolution. The length of this segment; which is the synthetic aperture, cannot exceed the real beamwidth of the radar at the ground since this is the maximum distance for which a given point on the ground remains illuminated for all points along the aperture. As such, contrary to the theory of the real aperture radar, whereby the largest possible antenna diameter is desired to minimize beamwidth, in stripmap mode it is desirable to have the radar beamwidth 
(as shown in light gray in Figure 1.1) as wide as possible and to let the aperture synthesis serve as the basis for cross-range resolution. The paper by Cutrona, et al [52], was the first to show that the maximum achievable cross-range resolution for a stripmap-mode SAR is

$$
\rho_{x}=\frac{A_{x}}{2}
$$

where $A_{x}$ is the actual physical width of the antenna in the along-track (azimuth) direction. This resolution is independent of either radar frequency or standoff range, both of which were limiting factors in the earlier real aperture radars. However, it is important to note that in satisfying the Nyquist sampling criterion, the pluses must be transmitted with the spacing along the flight path also equal to the resolution; that is, one half of the physical antenna width, in order to avoid aliasing due to undersampling. Consequently, the sample rate in cross-range, known as the pulse repetition frequency (PRF), may be rather high. Furthermore, while decreasing the antenna width improves cross-range resolution, it reduces the SNR because of the reduction in transmitted energy.

\subsubsection{Synthetic Aperture Radar in Spotlight-Mode}

The term "spotlight-mode" precisely describes the concept behind this particular SAR collection geometry. In spotlight-mode SAR, the radar is continually steered, or slewed, so as to constantly illuminate the same ground patch from every position along the synthetic aperture. This geometry is depicted in Figure 2.1. An advantage of spotlight-mode SAR over stripmap is that only the patch to be imaged is actually illuminated. Notice in Figure 1.1 that the illuminated area of the stripmap SAR (total shaded area) is significantly bigger than the area actually being imaged (dark gray). Since a spotlight-mode collection avoids this unnecessary illumination, the physical antenna width can be increased and the PRF reduced accordingly, thereby reducing along-track sampling requirements. Furthermore, because the radar beam is slewed, the illumination time is no longer dependent on (or limited by) beam width. 
The additional dwell time, in conjunction with the increase in gain from the wider antenna, reduces the microwave transmitter power requirements while increasing the system SNR and cross-range resolution.

Of course, the advantages of spotlight-mode over stripmap do not come without tradeoffs. Specifically, the patch size for a spotlight-mode collection is significantly smaller than that of stripmap because the radar beam is slewed in order to remain on a particular portion of the scene for the duration of the aperture. The polarformat algorithm (PFA), perhaps the oldest and most straightforward approach to processing spotlight-mode data, has an inherent shortcoming in its ability to deal with large scene sizes, in certain scenarios such as imaging at close range, at high resolution, or when utilizing a low center frequency. This shortcoming is based on the faulty assumption of planar EM wavefronts being transmitted by the radar, leading to the wavefront curvature degradation in PFA processed imagery. This dissertation introduces a method for the negation of these wavefront curvature effects for both squinted and broadside scenarios, thereby lifting the scene size limitations imposed by the polar-format algorithm. Thus, the necessity is avoided for multiple reference points, employed to precisely focus smaller spotlight mode patches. Consequently, the need to mosaic many smaller PFA processed patches into a larger scene is eliminated, as is the necessity to abandon the simplicity and advantages of PFA for another type of processing algorithm.

Note, too, that the length of the aperture, and thereby the aperture angle subtended $\Delta \theta$ (as shown in Figure 2.1), can not increase without bound. Consider a change in relative perspective wherein the radar platform is considered stationary and the imaged scene rotates relative to the platform. This is known as the inverse $S A R$ (ISAR) model, and is perhaps the simplest model for representing target motion in a spotlight-mode collection [12, pp. 72-75][53, 54]. As the aperture length in the spotlight-mode collection increases, points within the imaged scene "rotate" through a greater angle relative to the radar platform. During this rotation, the range-directed motion of this point can be plotted as a curve, referred to as the 
range curvature of this point, as is shown for the center reference point in Figure 1.4. When the range-oriented motion of a point exceeds that which is represented by the appropriate range bin of the digitally processed scene, then image defocus occurs. The polar-format algorithm compensates this movement in several ways, including the motion compensation of points in the scene relative to a single point (mocomp to a point) and by collecting the sampled data on an annular as opposed to a rectangular grid. In this way, the PFA processor becomes a partially focused radar system [55, pp. 4-6], and only the residual differential range curvature, or wavefront curvature, is left to deal with. Interestingly, it is the collection of reflected spotlight-mode range pulses on the polar annulus that forms the association between tomography and spotlight-mode imagery. This dissertation will describe an efficient and effective method for negating the defocus effects of wavefront curvature, via space-variant post filtering, thereby rendering the polar-format algorithm as a fully focused approach to spotlight-mode image formation.

The remainder of this dissertation deals strictly with aspects of spotlight-mode formation as they relate to the tomographic paradigm and its limitations. Specifically, shortcomings of the polar-format algorithm with regard to wavefront curvature anomalies, which will be addressed and resolved (hopefully to a satisfactory degree) within this paper. While in some circumstances, spotlight-mode algorithms are used to process stripmap collections, in general the paradigm and processing procedures for stripmap SAR differ substantially from those of spotlight-mode. The reader is directed to the following textbook references for excellent treatments of stripmap SAR: [56, 57, 58, pp. 441-482] and [59, pp. 268-284]. The papers [54,60,61, 62] also address stripmap SAR in detail. 
Chapter 2. Aperture Synthesis, Tomography and Polar-Formatting

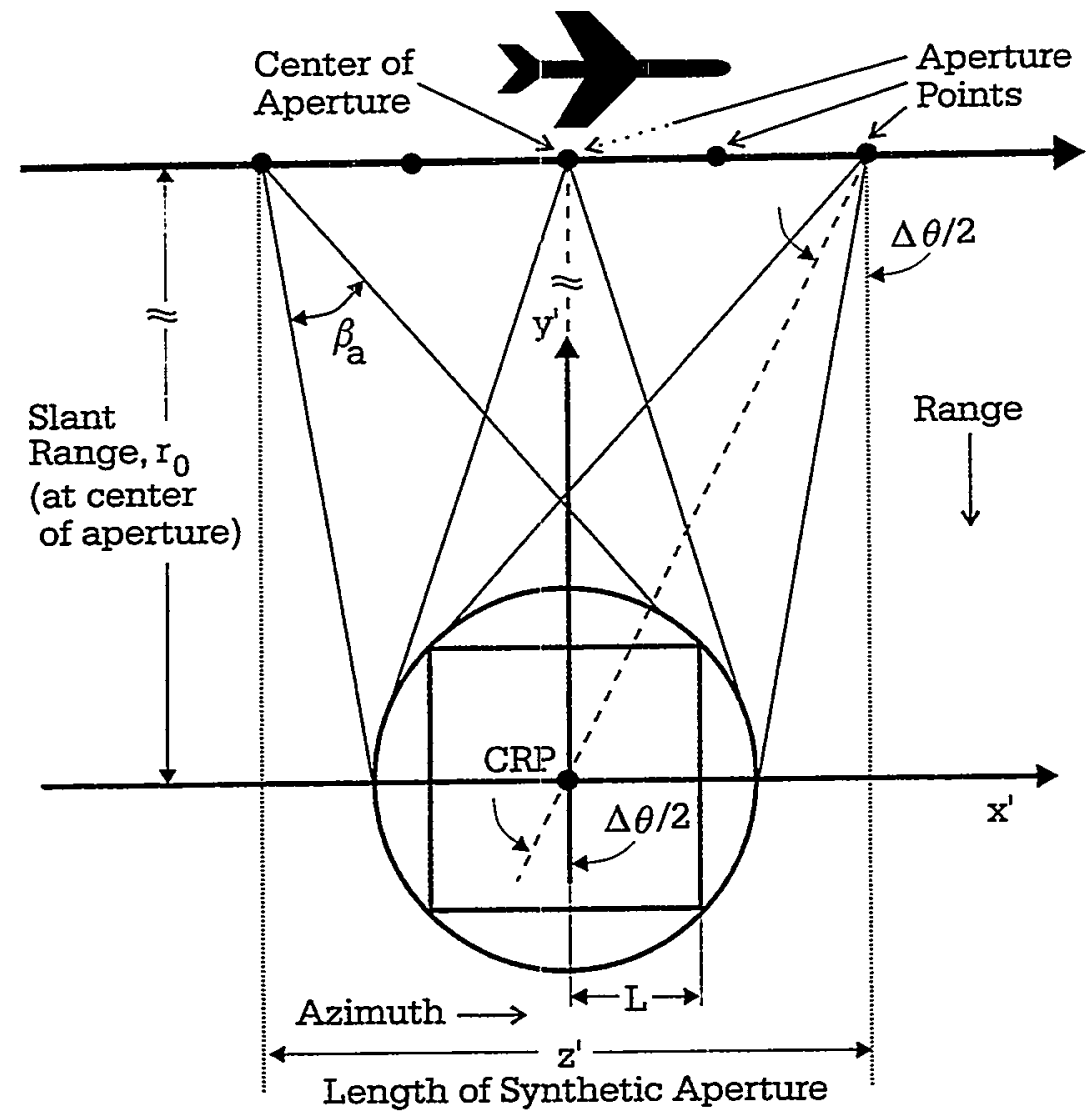

Figure 2.1: Beamwidth, Aperture Extent and Patch Radius in Spotlight-Mode

\subsection{Spotlight-Mode Tomography and Polar- Formatted Data}

It is not difficult to imagine a relationship between computerized axial tomography (CAT), wherein an object is imaged through the processing of projectional views over a set of measurement angles, and spotlight-mode SAR, which collects the reflected samples of a scene from different positions along a trajectory. Indeed, while CAT and spotlight-mode SAR have developed independently, they share a remarkable similarity of principle, namely the projection-slice theorem. The CAT scan, which enables the imaging of two-dimensional cross sections of solid objects, is used extensively for the examination of internal organs and the nondestructive testing of manufactured 
items, as well as many other applications [63]. While its roots lie in the integration of $\mathrm{x}$-ray projections, as first described by Johan Radon (though abstractly and not related to x-rays) in 1917 (an English translation of which is found in [63]), the analogy between tomographic imaging and the two-dimensional radar reflectivity function of spotlight-mode SAR was given by Munson and his colleagues in 1983 [4]. In his paper, Munson states that the spotlight-mode SAR can be interpreted as a tomographic, band-limited reconstruction problem and that the signal processing theory is characterized in terms of the projection-slice theorem, as opposed to the traditional radar view of Doppler filtering.

In order to cast spotlight-mode SAR as a tomographic problem, the projectionslice theorem must be mathematically described, and more importantly, its meaning must be understood as it relates to the spotlight-mode SAR concept. First, consider the two-dimensional (2D) Fourier transform pair:

$$
G(u, v)=\int_{-\infty}^{\infty} \int_{-\infty}^{\infty} g(x, y) e^{-j 2 \pi(u x+v y)} d u d v
$$

and

$$
g(x, y)=\int_{-\infty}^{\infty} \int_{-\infty}^{\infty} G(u, v) e^{j 2 \pi(u x+v y)} d x d y
$$

where $g(x, y)$ represents the 2D complex reflectivity function of the ground patch being imaged and $G(U, V)$ is the Cartesian Fourier transform of $g(x, y)$. Scale factors are not relevant to the development and are ignored. It can be shown that $G(\rho, \theta)$ is the polar representation of the 2D Fourier transform of $g(x, y)$ for an arbitrary angle $\theta$ (as proven in numerous references, including [7, pp. 55-57]). Therefore, the inverse Fourier transform of Equation (2.7) for projection angles spanning 180 degrees $(0 \leq \theta \leq \pi \mathrm{rad})$, can be written as

$$
g(x, y)=\int_{0}^{\pi} \int_{-\infty}^{\infty} G(\rho, \theta) e^{j 2 \pi(\rho x \cos (\theta)+\rho y \sin (\theta))} d \rho d \theta
$$

where $\theta$ is measured with respect to the positive abscissa axis, as shown in Figure 2.2. If a variable $u$ is defined such that

$$
u=x \cos (\theta)+y \sin (\theta),
$$


then Equation (2.8) becomes

$$
g(x, y)=\int_{0}^{\pi} \int_{-\infty}^{\infty} G(\rho, \theta) e^{j 2 \pi \rho u} d \rho d \theta
$$

Next, if the projection function $p_{\theta}(u)$ is defined as

$$
p_{\theta}(u)=\int_{-\infty}^{\infty} G(\rho, \theta) e^{j 2 \pi \rho u} d \rho
$$

then Equation (2.10) becomes

$$
g(x, y)=\int_{0}^{\pi} p_{\theta}(u) d \theta
$$

and by Fourier transformation,

$$
P_{\theta}(U)=\int_{-\infty}^{\infty} p_{\theta}(u) e^{-j 2 \pi u U} d u
$$

This is the mathematical development for the projection-slice theorem, which states that the one-dimensional Fourier transform of a projection function $p_{\theta}(u)$ is equal to the two-dimensional Fourier transform $G(u, v)$ of the image to be reconstructed, evaluated along a line in the Fourier plane that lies along the same angle $\theta$ measured with respect to the positive abscissa axis. In other words, $P_{\theta}(U)$ is a one-dimensional (1D) slice at angle $\theta$ of the 2D Fourier transform of $g(x, y)$, as shown in the geometric projection model of Figure 2.2. This analysis forms the basis of the convolution back-projection image formation method (CBP), as well as the polarformat Fourier inversion algorithm (PFA). As projections are taken over a range of $\theta$, the 1D Fourier transform values of the projection data determine values of the 2D Fourier transform $G(u, v)$, along lines of the same angular orientations. The projections must be taken at sufficiently close intervals along the synthetic aperture as to obey the Nyquist sampling criteria for the Fourier region of support in the cross-range direction. This sampling rate is the pulse repetition frequency, or PRF. Similarly, each projection must adequately sampled in order to support the bandwidth in range (typically referred to as the $A / D$ sampling rate). In neither case is the scene resolution a function of sampling rates, but instead that of the chirp 
bandwidth for range, and the induced bandwidth in terms of the angular extent $\Delta \theta$; for cross-range. Thus, the tomographic view of the scene turns out to be a bandpass filtered projection of the ground patch reflectivity, where the scene resolution is determined by the frequency extent of the data, in both range and azimuth, before Fourier inversion into the reconstructed image. Since the limited duration of the transmitted radar pulse implies that $P_{\theta}(U)$ will only be determined on a restricted interval of spatial frequencies, each radial segment of the Fourier data will be of the same length. Consequently, the Fourier samples will be constrained to a circular (polar) annulus, with a nominal radius $k_{0}$, as offset from the origin, of

$$
k_{0}=\frac{2 \omega_{0}}{c}=\frac{4 \pi}{\lambda}
$$

where $\omega_{0}$ is the radar center frequency and $\lambda$ is the corresponding wavelength. The frequency extent (spatial bandwidth) $\Delta Y^{\prime}$ in the range dimension is found to be

$$
\Delta Y^{\prime}=\frac{2}{c}\left(2 \pi B_{c}\right)
$$

where $B_{c}$ is the chirp bandwidth as defined in Equation (2.2). The nominal crossrange extent, as determined by the radius $\frac{4 \pi}{\lambda}$ and the angular extent $\Delta \theta$ of the annulus is

$$
\Delta X^{\prime}=2\left(\frac{4 \pi}{\lambda}\right) \sin (\Delta \theta / 2)
$$

As is common with spotlight-mode SAR collections, the angular diversity $\Delta \theta$ is typically very small, and under the small-angle assumption of $\sin (\theta)=\theta$, the expression for $\Delta X^{\prime}$ can be approximated by

$$
\Delta X^{\prime}=\frac{4 \pi}{\lambda} \Delta \theta .
$$

These parameters are depicted in the spotlight-mode, Fourier collection surface of Figure 2.3.

In the case of a straight-line flight path across the synthetic aperture, the collection surface swept out in three-dimensional Fourier space is simply a plane, referred 


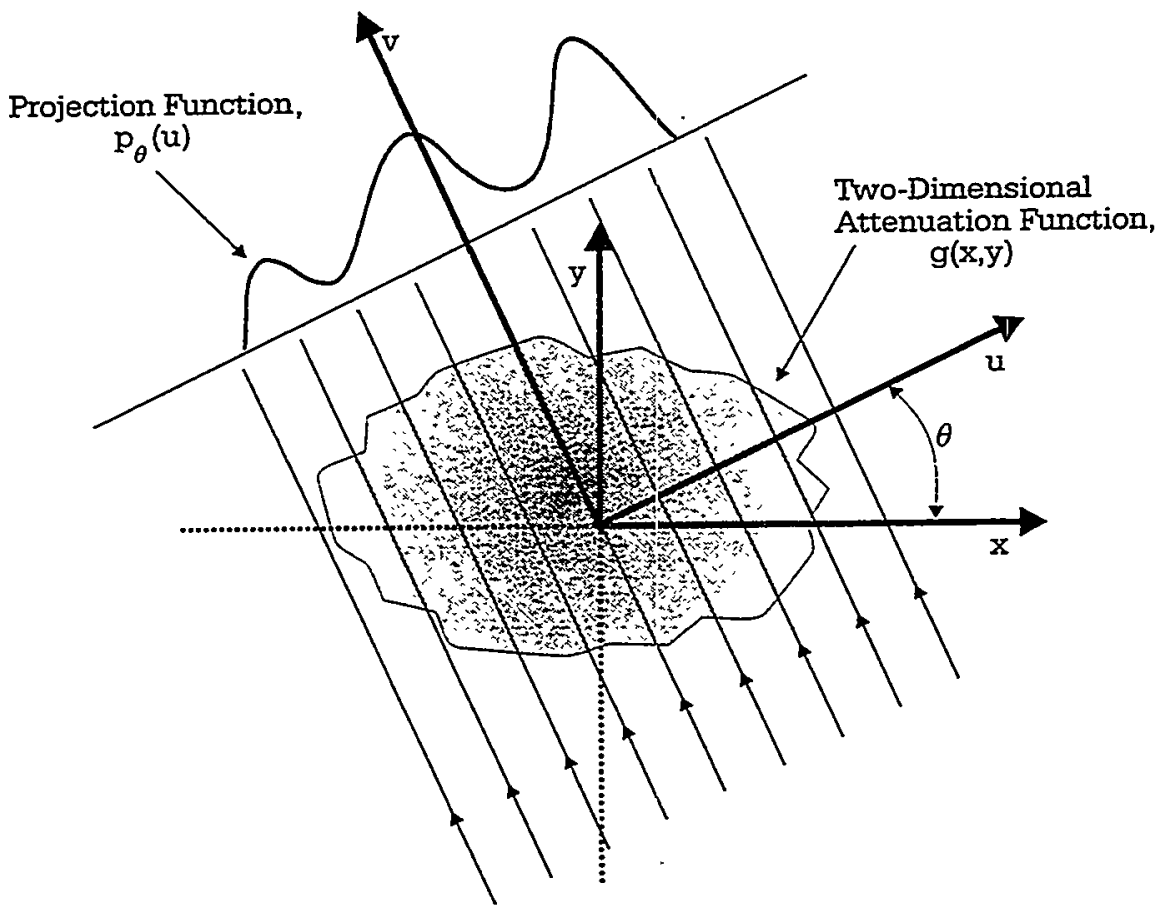

(a) Spatial Domain

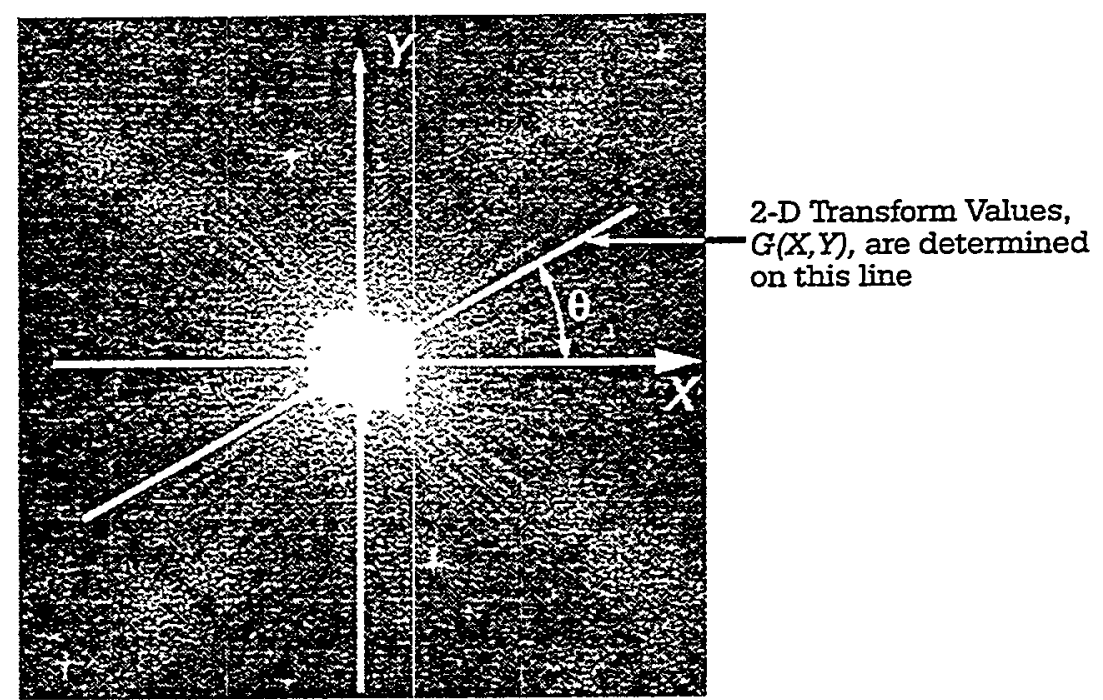

(b) Frequency Domain

Figure 2.2: Projectional View of X-ray Tomography 


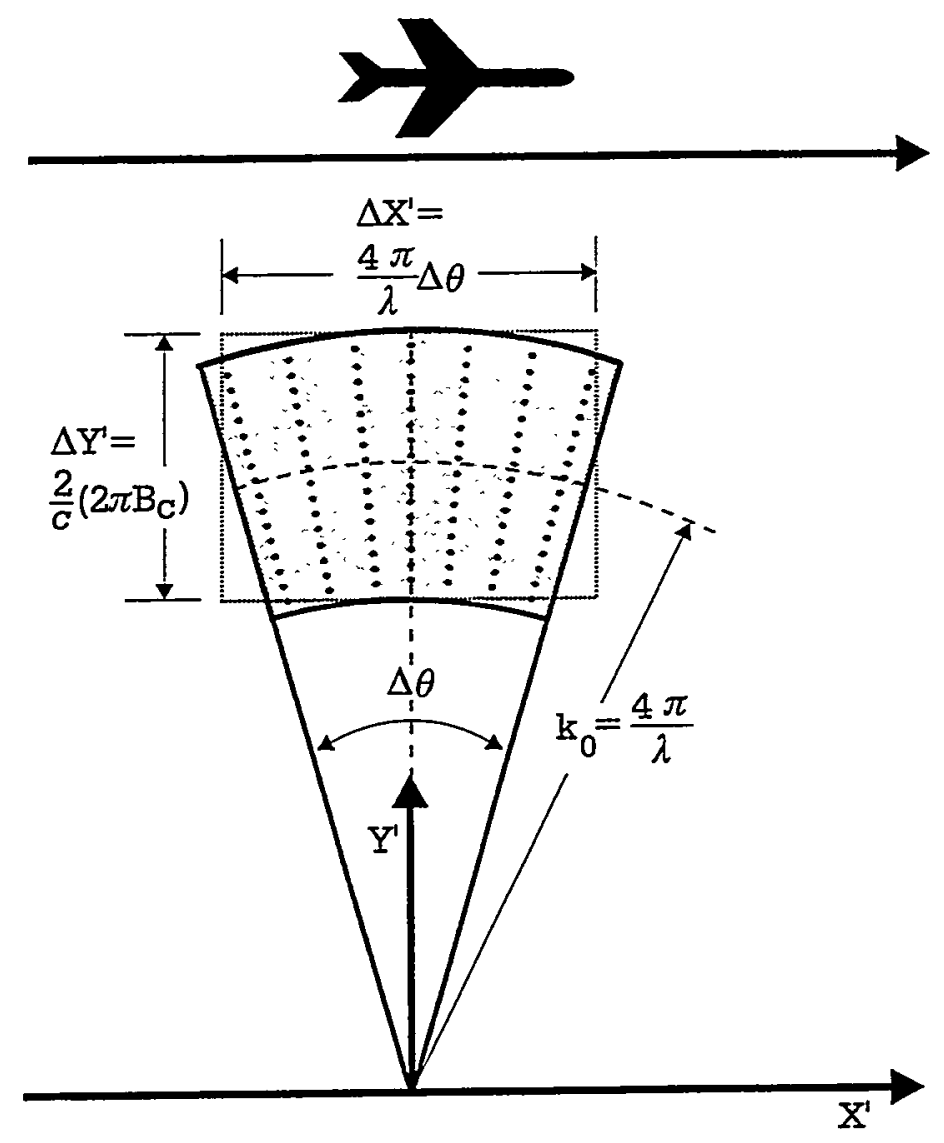

Figure 2.3: Slant Plane Fourier-Domain Samples and Frequency Extent

to as the slant plane. This straight-line assumption models the situation for a typical airborne collection where the out-of-plane motion is insignificant, and it will be adopted throughout this dissertation. In the case of uncompensated platform motions, or if the collection is spaceborne, the surface swept out is 3D ribbon as opposed to a plane. The ramifications of this motion on wavefront curvature (and its compensation) are beyond the scope of this dissertation, but will be briefly addressed in the "Future Work" section of Chapter 7. For the straight-line motion assumption that has been adopted, the slant plane is determined by the line of the flight path and the aim point (center reference point, or CRP) of the scene.

As was originally obtained by Walker through Doppler frequency analysis, the slant plane surface is a two-dimensional Fourier slice of the $3 \mathrm{D}$ scene reflectivity 
function [2]. However, this result was also shown by Munson via his tomographic analysis. Though not analyzed or considered by Munson, Jack Jakowatz and his colleague Paul Thompson, through a novel 3D tomographic model, demonstrated the data within the 2D Fourier surface to be the orthogonal projection of the ground scene onto the slant plane [6]. Since ground plane objects typically have height, this model predicts the range-dependent layover that affects these 3D objects when projected into the slant plane. Furthermore, it describes perturbations in the phase of waves reflected from point targets, which are indicative of terrain height variations. Such is the foundation for interferometric $S A R$, as originally proposed in [64], and further described in the article [65] and the books [12, pp. 367-383] and [7, pp. 273-351]. With regard to plane-to-plane transformations, the 2D annular slice of Fourier-domain data, often referred to as the phase-history, represents the orthogonal projection of the ground plane scene into the slant plane, as described by the $3 \mathrm{D}$ model. Thus, the phase-history domain description of a slant plane collection is a set of samples lying on a polar raster imposed on an annulus in the slant plane, as shown in the upper plane of Figure 2.4. It may be easiest to consider a set of coordinate axes $\left(X^{\prime}, Y^{-1}\right)$, wherein the center pulse of the aperture is used to define the $Y^{\prime}$ direction and the $X^{\prime}$ axis is orthogonal and lying in the plane. The $Y^{\prime}$ dimension corresponds to the slant range spatial frequencies, and $X^{\prime}$ to the cross-range frequencies in the slant plane, such that the $2 \mathrm{D}$ inverse Fourier transform of the data produces an image in a slant plane domain with axes of $x^{\prime}$ and $y^{\prime}$.

Alternatively, with a change in perspective, the slant plane image data can be projected onto the ground plane having coordinate axes $x$ and $y$, and ground-projected phase history samples are in $X$ and $Y$, as shown in the bottom plane of Figure 2.4. In the event of a broadside collection, the projection operation simply imposes a contraction of phase history data onto the ground plane in the range direction, by a factor of $\cos (\psi)$, with no contraction in the cross-range direction. For squinted collections, the slant plane is actually tilted relative to the broadside slant plane surface. In this case, there exists a squint dependent contraction in both range and azimuth, 
when projecting from the slant plane to the ground plane. This greatly complicates the process of projecting from one of these planes to the other. Furthermore, the calculation of range dependent layover for $3 \mathrm{D}$ targets is less straightforward. The motivation for forming the SAR image in the ground plane as opposed to the slant plane is that the ground plane image is orthographically correct for all nonelevated targets (although targets with height still exhibit range-oriented layover). This property is often helpful in the human interpretation of imagery. Unless otherwise noted, this dissertation assumes the simpler slant plane perspective, and as such, this perspective will be used for all calculations and derivations. Consequently, slant plane to ground plane transformations (and their associated complications) become unnecessary. While in a practical sense, these projections are important to the interpretability of imagery, they are ancillary to the wavefront curvature analysis being presented.

The image domain resolutions in the slant plane are easily obtained from the spatial-frequency bandwidths of Equations (2.15) and (2.17), as shown in Figure 2.3. The resulting range resolution is

$$
\rho_{y^{\prime}}=\frac{2 \pi}{\Delta Y^{\prime}}=\frac{c}{2 B_{c}}
$$

while the cross-range resolution (considering the small-angle approximation) is

$$
\rho_{x^{\prime}}=\frac{2 \pi}{\Delta X^{\prime}}=\frac{\lambda}{2 \Delta \theta}
$$

Since the radar bandwidth can never exceed twice the center frequency, these resolution expressions have lower bounds. Specifically, Equation (2.18) dictates that range resolution can never be better than $\lambda / 4$, while Equation 2.16 imposes a maximum $\Delta X^{\prime}$ of $8 \pi / \lambda$ (for a $\Delta \theta$ of $180^{\circ}$ ), and by Equation (2.19), the cross-range resolution is also bounded by $\lambda / 4$. For a number of practical reasons, SAR systems typically do not achieve resolutions even close to these theoretical bounds. In the case of ground plane resolution, the uniform contraction of the phase history data in the range direction (for broadside collections) results in a corresponding ground range 
Chapter 2. Aperture Synthesis, Tomography and Polar-Formatting

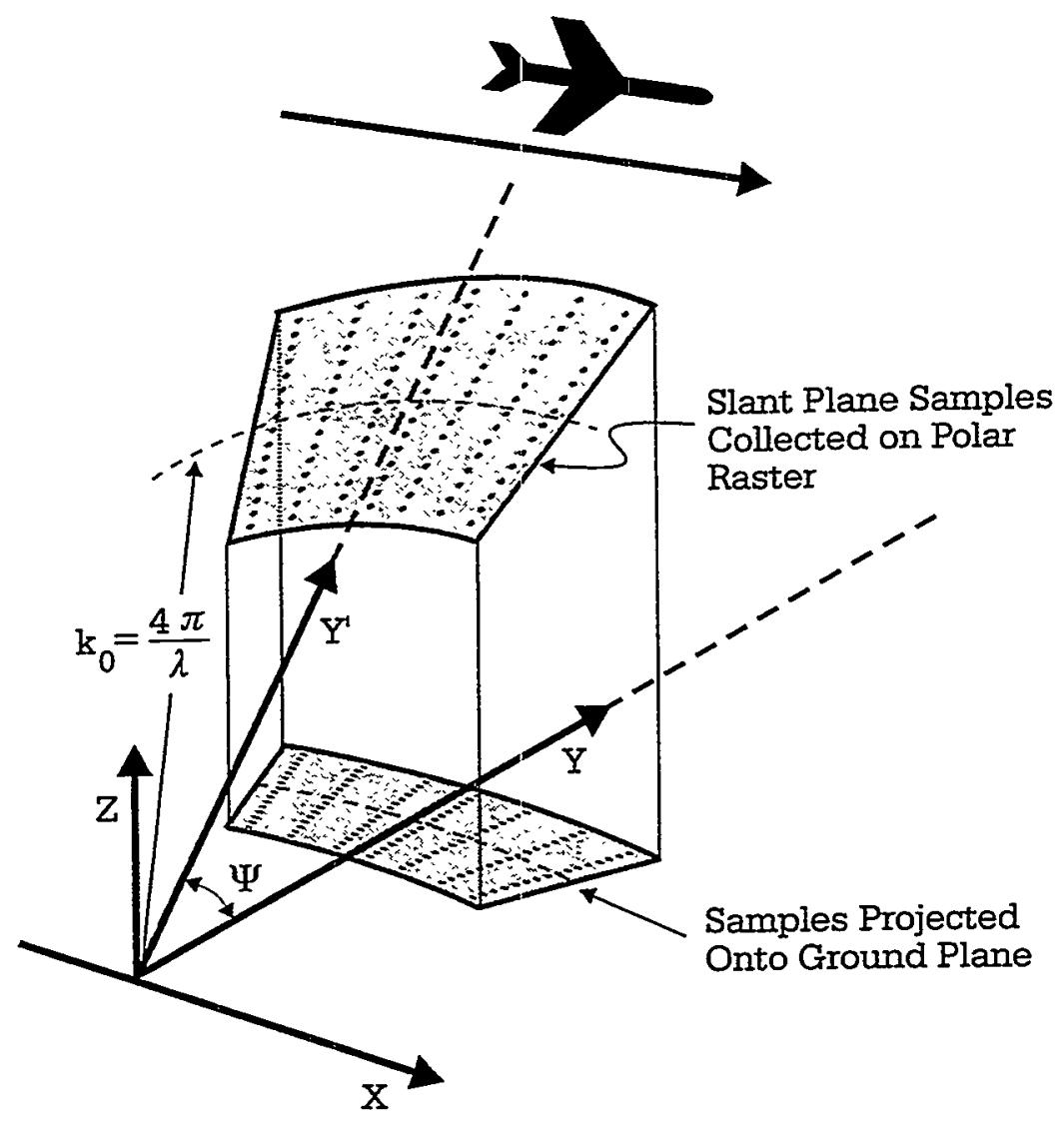

Figure 2.4: Slant Plane Fourier-Domain Samples and Ground Plane Projection for Spotlight-Mode

resolution scaling of $\cos (\psi)$. The ground range resolution is then

$$
\rho_{y}=\frac{2 \pi}{\Delta Y}=\frac{c}{2 B_{c} \cos (\psi)}=\frac{\rho_{y^{\prime}}}{\cos (\psi)}
$$

while the ground cross-range resolution remains unchanged from its slant plane counterpart and is found to be

$$
\rho_{x}=\frac{2 \pi}{\Delta X^{\prime}}=\frac{2 \pi}{\Delta X}=\frac{\lambda}{2 \Delta \theta}=\rho_{x^{\prime}}
$$

It is important to realize that the difference in value of $\rho_{y}$ and $\rho_{y^{\prime}}$ does not imply that the resolvability of the two targets is improved in a slant plane as opposed to a ground plane reconstruction. This is due to the corresponding reduction in spatial distance between the targets as imaged in the slant plane. Thus, the only difference 
between ground and slant plane image reconstructions for broadside collections is the scaling of the range dimension.

As the synthetic aperture is flown, only a limited number of projections can be acquired. These must be collected at a rate that satisfies the Nyquist criteria for the cross-range bandwidth, as determined by the total aperture extent $\Delta \theta$. Similarly, each projection must be sampled in range as to satisfy the Nyquist criteria for the given range chirp bandwidth. Given the distance of the radar platform from scene center, even a few degrees of aperture translates into very long collection (flight) distances. Consequently, measurements are possible only for limited angular extents. Despite these bandlimiting processes, the reconstructed images are observed to contain surprisingly few artifacts of bandlimiting. This is remarkable, given that the basic considerations of Fourier processing and the limited slice of Fourier data transduced make it hard to imagine any usable image being formed at all. Furthermore, this is in direct contrast to $\mathrm{x}$-ray tomography, which requires a full circular view of the object being imaged. However, for SAR, a coherent system in that it considers both phase and magnitude of the reflectivity function, it is found that an assumed distribution of point scatters in the image, each having random phase, serves to disperse frequency components over much of the transform plane. That is, the image domain microwave reflectivity function typically possesses a phase function that is essentially uncorrelated. This particular effect was first described by Munson and Sanz in [66], and is attributed to the coherent speckle properties of SAR imagery. This property states that because of the small slice of Fourier data being considered, the reconstruction is actually a speckled version of the scene reflectivity that does not exactly match the true magnitude of the function point-for-point.

Tomographic imaging and SAR bear enough resemblance to each other for both systems to be explained in terms of the projection-slice theorem. However, perhaps their biggest difference lies in the bandlimited reconstruction of the SAR image, as compared to tomography. Furthermore, there exist other distinct differences between SAR and tomographic imaging. For instance, while x-ray tomography trans- 
duces image-domain, transmissive, real data centered at baseband, SAR transduces complex, reflective, Fourier data offset in spatial frequency by $4 \pi / \lambda$ (in the slant plane). Furthermore, the SAR Fourier data are only determined over an annulus that is angularly narrow, yet the phenomenon of coherent speckle ensures suitable reconstruction of the bandlimited image. In contrast, views spanning a full $180^{\circ}$ are employed in medical CAT imaging. While Munson's tomographic model makes these contrasts and comparisons, it fails to consider the effects of target height and terrain height variations, as described above. It will be seen next, when spotlight-mode data is directly Fourier inverted (such as with the polar-format algorithm), that the planar wavefront assumption of Munson's model induces serious wavefront curvature effects in certain imaging scenarios. The rest of this dissertation will address the affects of the planar wavefront assumption on polar-formatted spotlight-mode SAR imagery, and will present a method for the compensation of these effects; namely, the space-variant post-filter.

\subsection{The Polar-Format Algorithm}

The essence of the polar-format algorithm is the Fourier inversion of the frequency domain spotlight-mode data (phase-history) collected on a polar raster. As such, it is considered a direct Fourier inversion method. As described in the previous section, the annular Fourier data represents the orthogonal projection of the threedimensional complex scene reflectivity onto the two-dimensional slant plane. The beauty of the polar-format algorithm lies in its simplicity, as described by the tomographic paradigm, and in its straightforward implementation. However, it also suffers the shortcomings of this paradigm; namely, the visible effects of wavefront curvature errors in formed imagery, as a consequence of the planar wavefront assumption on which it is based. Specifically, since the derivation of the PFA technique relies upon the unrealistic assumption of strictly planar wavefronts in the transmitted microwave pulses, the polar-format algorithm cannot compensate these wavefront 
curvature effects. Consequently, any actual amount of curvature present in these wavefronts introduces the geometric distortion and space-variant defocus present in the formed imagery for certain imaging scenarios, such as those at close range, low center frequencies, high resolutions, or when imaging large patches. Nevertheless, the polar-format algorithm one of the most popular Fourier inversion methods of spotlight mode image formation because of its simplicity, established reputation in the remote sensing community, and ability to form images of large scenes without resorting to subpatch processing.

With the addition of space-variant post-filtering for wavefront curvature correction, as presented in this dissertation, the major factor that has restricted scene size is finally lifted. The fundamental steps of the polar-format algorithm are outlined in Figure 2.5, and are briefly described below. For the reader with a deeper interest in the specifics of the polar-format algorithm, detailed information can be found in a number of references, including Walker's original treatment of the subject in $[1,2,3]$, and more recently, a book in which PFA is the focus of its spotlight-mode image formation techniques [7]. In Chapter 6, PFA will be compared to other popular image formation algorithms in terms of computational burden, and it will be shown that PFA, in conjunction with space-variant post-filtering for wavefront curvature correction, is competitive with (or superior to) other popular methods for many imaging scenarios.

Before Fourier inversion into the image domain, interpolation of the annular Fourier data onto a Cartesian rectangular grid is typically performed. This is necessary because there currently exists no efficient, fast Fourier transform (FFT) routine that operates directly on polar-oriented data (although some efficient polar approximations to the Cartesian FFT have been proposed in $[67,68])$. Without prior interpolation to a grid, the Fourier transformation of polar raster data results in significant space-variant defocus for imaging scenarios where the data are not already near-Cartesian, as in the case of high resolution imagery or when using low center frequencies (due to the shortened nominal annular radius, $k_{0}$, as shown in Figure 
2.3). While in general, very low resolution imagery over small patches can be formed without first performing polar-to-rectangular resampling, this technique becomes impractical at best for the high resolution imaging situations for which spotlight-mode is intended. Without this resampling, a gradual loss of focus is observed as a function of distance from the patch center, and for most practical SAR applications this approach is totally inappropriate.

The book by Jakowatz, et. al [7] derives an equation for the maximum imaged patch size as a function of radar wavelength and resolution, when the polar-torectangular interpolation (polar-formatting) step is avoided. For a given slant plane range and cross-range resolution of $\rho_{y^{\prime}}$ and $\rho_{x^{\prime}}$, respectively, the maximum allowable slant plane patch radius $x_{0}^{\prime}$ is defined by the inequality

$$
\left|x_{0}^{\prime}\right| \leq \frac{2 \rho_{x^{\prime}} \rho_{y^{\prime}}}{\lambda}
$$

where $\lambda$ is the radar wavelength and $\pi / 4$ is the maximum allowable quadratic phase error leading to the defocus. This phase error limit is such that defocus is kept to within subpixel levels (see Appendix A). Other authors arrive at the same constraints using somewhat different methods. One such alternative derivation is presented in [2] and another in [69]. An example of the severity of this constraint is a SAR at 10 Ghz $(\lambda=3 \mathrm{~cm})$ with sufficient bandwidth and angular diversity to resolve 1 meter in range and cross-range $\left(\rho_{y^{\prime}}=\rho_{x^{\prime}}=1 \mathrm{~m}\right)$. Based on Equation (2.22), this system can only image a patch of 130 meters square, without exceeding the $\pi / 4$ quadratic phase error limit when polar-formatting is not implemented. Thus, unacceptable restrictions are required when not polar-formatting the phase history data. This is because the polar-to-rectangular interpolation preserves the mitigation of range cell migration as accomplished via sampling the original phase history on a polar raster.

While the polar-to-rectangular interpolation lifts these scene size restrictions, this flexibility does not come without a price, as interpolation requirements are severe in terms of required accuracy as well as computational burden. The reason that the interpolation requirements are so severe is due to the nature of the Fourier domain 
(a)

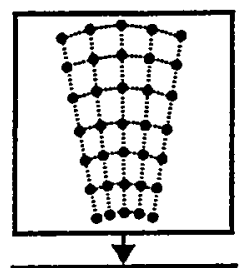

Polar-Format

Phase History

(b)

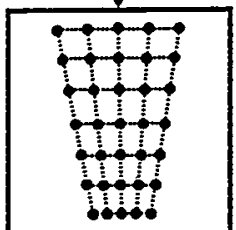

Phase Stabilization

Range Deskew

Out-of-plane Correction

Range Interpolation

(c)

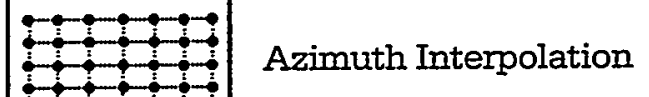

(d)

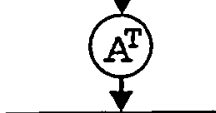

(Transpose)

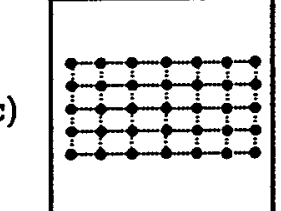

Azith Interpolation

(e)

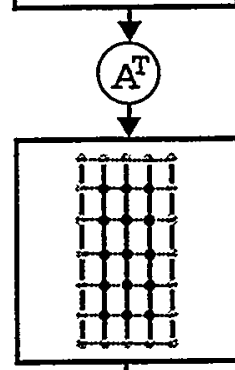

(Transpose)

$\frac{1}{A^{\mathrm{T}}}$

Aperture Weighting

Range FFT

(f)

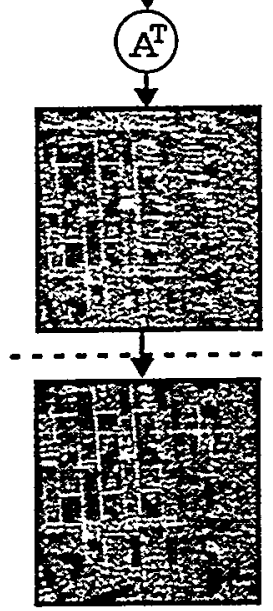

(Transpose)

Autofocus

Azimuth FFT

Geometric Warp

Image Equalization

NEW:

Space-Variant

Post-Filtering for

Wavefront Curvature

Correction

Figure 2.5: Polar-Format Processing Algorithm - Detailed Steps 
in which the data are interpolated. Every point in the Fourier domain contributes to the spatial reconstruction and consequently, single errors in the Fourier domain affect the entire spatial domain. Therefore, it is important that the interpolator be as accurate as possible, particularly in the spectral regions where energy is concentrated. One notion of the theory of resampling and interpolation is that the process can be viewed as a linear digital filtering operation. The interpolation process must maintain the underlying signal integrity and not introduce significant errors of its own. Specifically, since data are interpolated in the complex-valued phase history domain, the interpolation filter must be linear in its phase response so as not to introduce phase errors, and it must also meet certain bandwidth and Nyquist conditions. In the particular instance of spotlight-mode SAR, the resampling process is that of a $2 \mathrm{D}$ interpolation of the polar-format data to Cartesian coordinates, so that fast Fourier data inversion methods may be implemented to form the images.

Although the ideal solution (in terms of interpolation accuracy) is to perform true two-dimensional interpolations, this approach is computationally intensive. Instead, a less computationally burdensome method exploits separability, whereby one-dimensional interpolations are performed in the range direction (along radial samples) for each radar pulse, followed by one-dimensional interpolations in the cross-range dimension for each range line of the Fourier data $[5,36,49]$. Known as the keystone method [70], the data are first interpolated in range, from their original uniformly spaced sample grid onto a new uniformly spaced grid to form a keystone, as shown in Figure 2.5(b). Next, the data are interpolated in the cross-range direction, resulting in the rectangular sample grid as shown in Figure 2.5(c) This step is identical to that for the range-oriented samples, except that the data lying along any given cross-range line are unequally spaced. These keystone samples are more closely spaced at the samples near mid-aperture and increase in separation towards the outer edges of the phase history. Furthermore, the sample separation increases with distance from the polar origin. These are the consequences of using separable ID resampling, when in reality, the process is only separable in the polar Fourier domain 
$(r, \theta)$ and not in the rectangular domain of $(U, V)$. Thus, while more computationally efficient as a result of separability, this interpolation approach is not without its own inherent inaccuracies, and the interpolation filters must carefully accommodate this space-varying sample spacing during ID cross-range interpolation.

In practice, for most spotlight-mode SAR data collections, the variation in sample spacing along an individual cross-range record is small because the angular extent of the phase history is only a few degrees. As such, on an individual cross-range record, the interpolation filters can act as if the sample spacing is uniform without introducing intolerable errors. This is in contrast to CAT imaging; whereby large viewing angles (typically $180^{\circ}$ ) prohibit the use of separable $2 \mathrm{D}$ interpolation. In these CAT imaging scenarios, convolution back-projection is often implemented as the image formation algorithm of choice. Furthermore, while SAR image formation may be considered a tomographic process, the polar-format algorithm is often unfairly criticized for having unreasonable interpolation errors [71,72], which is not the case when applied to most SAR imagery (the exception being UWB SAR, as described further in Chapter 6). It is important to realize that for spotlight-mode SAR, whereby the angle of aperture extent $\Delta \theta$ is typically small and the Fourier data are offset significantly from baseband, the less computationally burdensome polar-format algorithm can very effectively form images since the polar-to-rectangular interpolation step results in a negligible amount of interpolation error [33]. It has been pointed out in several papers including [71,73], the resampling portion of the polar-format algorithm can be computationally burdensome. In fact, since very precise interpolations are required to maintain image quality, the $2 \mathrm{D}$ interpolation is typically the dominant factor in the overall computational complexity of the polar-format algorithm. However, in spite of the burden of the $2 \mathrm{D}$ interpolation, the polar-formatting algorithm still has a lower overall computational complexity than convolution back-projection.

It has been suggested that the problem of interpolation and resampling can be reduced to a digital filtering operation. The sinc function can be thought of as the impulse response of the ideal digital filter. However, the sinc impulse response must 
be approximated with one that is realizable in that it has no infinite sums, yet preserves phase and provides suitable passband and stopband characteristics. Typically, truncated versions of infinite length sampling functions are utilized, with the number of zero crossings (in the case of the sinc) selected to form a tradeoff between interpolation error and computational burden. Specifically; the rate of falloff of passband and stopband ripple is determined by the filter length, as is the transition band steepness. Stopband attenuation must be sufficient to mask the Nyquist aliasing that occurs from the folding of energy back into the filter. Meanwhile, the magnitude of the ripple is a result of windowing (truncating) the filter function with a rectangular window. Consequently, in order to reduce this ripple, tapered window functions (eg: Hamming or Taylor) are typically implemented. This is referred to as the weighted interpolator. Alternative interpolator designs, based on polynomial or higher order functions, are also applicable to SAR. The design of such filters is beyond of the scope of this paper, though their computational burden will be discussed (in a most general sense, for the weighted sinc interpolator) in Chapter 6. Furthermore, Fourier data interpolation in SAR imagery is a heavily researched topic and the reader is directed to the following excellent references on the subject: [7, pp. 133-171],[74, pp. $117-162]$, and $[75,76]$.

The interpolation process, in conjunction with Fourier transformation, comprise the essential elements of polar-format spotlight-mode image formation. However, from a practical sense, there are often a number of additional steps that are taken to ensure satisfactory image quality. Obviously, the issue of wavefront curvature correction takes center stage in this dissertation as one of the few remaining hurdles in polar-formatted SAR imagery. In fact, while it is a difficult problem which has motivated this dissertation research, other serious image quality issues exist in polarformatted spotlight-mode SAR imagery. These are attributable to assumptions in the tomographic paradigm on which it is based, as well as geometric and atmospheric anomalies that occur in real imaging scenarios. However, research has led to many practical and efficient solutions to these problems, and as such, these will issues will 
not be treated extensively here, but still deserve brief mention. These steps are shown in Figure 2.5 and are as follows:

- Phase history acquisition - The phase history data are acquired on a polar raster with the necessary angular extent, chirp bandwidth and sample rates to achieve the desired resolution and patch size.

- Phase history trim - The polar phase history data are trimmed to a rectangle (though still in polar format), either by inscribing, exscribing, or a combination of the two. This process helps to control the IPR sidelobe structure.

- Range deskewing - A deskewing of range pulses is performed to remove the effects of the residual video deramp phase term, if required. This compensates the geometric distortion and defocus in scenarios where residual deramp phase is significant.

- Motion compensation to a point - The phase history pulses are properly demodulated and have been properly motion compensated so that the phase of a hypothetical target at the patch center is constant over the entire synthetic aperture time.

- Geometric transformation - The phase history is geometrically transformed to the desired imaging plane, and known three-dimensional (out of plane) motion is compensated on a pulse by pulse basis, given knowledge of the platform position (via the pointing vector generated during the collection).

- Two-dimensional interpolation - A 2D interpolation is performed, as previously described, with the appropriate filtering/downsampling to end up with Cartesian samples at the desired density over the rectangular area selected.

- Windowing (aperture weighting) Fourier data - The appropriate window functions (eg: Taylor, Hamming or apodization) are applied to the Cartesian 
Fourier data so that the desired sidelobe reduction will be achieved in the final image [12, pp. 507-524] [46, pp. 225-239] [77].

- Fourier transformation - A 2D FFT is applied to Fourier invert the phase history data, thereby producing the complex-valued reconstructed spotlightmode image. Appropriate zero padding of the phase history prior to inversion helps to achieve the desired image domain oversampling ratio. This helps in "pleasing" the human visual system during interpretation.

- Autofocus - A focus procedure is applied, typically after range Fourier transformation (compression) and prior to cross-range Fourier transformation, to compensate unknown platform errors and certain propagation errors. One typical procedure is phase gradient autofocus (PGA) [78, 79], and another is subaperture autofocus [80], both of which operate in the range-compressed, Cartesian domain.

- Geometric warping - A simple geometric warp is performed on the complex image data to mitigate the distortion induced by wavefront curvature.

- Detection - The complex, image-domain data are magnitude-detected (via a square root or logarithmic procedure) and a viewable, grayscale binary image is produced. This image is then contrast-adjusted, equalized, or gray-scale mapped as required for visual interpretation.

- New: Wavefront curvature defocus compensation - A Space-variant post-filter is applied to the complex-valued image to mitigate the defocus effects of wavefront curvature (if necessary). The post-filtered, complex-valued image is then magnitude-detected so it can be displayed.

The book by Jakowatz, et. al [7, pp. 105-270] gives more detailed information on all the PFA steps previously mentioned. 


\section{Chapter 3}

\section{The Planar Wavefront Assumption in Polar-Formatted Imagery}

The tomographic paradigm for spotlight-mode SAR imaging; as previously described, presents the concepts of SAR in a clear and concise way within a signal processing framework. However, there are certain limitations to the scope of the tomographic description of spotlight-mode SAR. In fact, such would be the case for any description of the SAR imaging process that portrays the collected SAR data as some 2D Fourier projection of the 3D scene function. This is true of Walker's original spotlight-mode SAR description $[1,2,3]$, and extends to the analogy of spotlight-mode SAR as a tomographic process, as presented by Munson [4] and later Ausherman [5]. This chapter describes the most significant current limitation of the polar-format algorithm as it relates to the tomographic paradigm. That is, the faulty assumption of planar wavefronts illuminating the imaged scene. A simple model will be presented which explains the geometric distortion induced by this assumption, with an accompanying analysis to describe the conditions under which this behavior is troublesome. Examples of geometrically distorted images are also presented. An introduction to the associated quadratic defocus is given, as well as illustrative examples, with appropriate scene size limitations being discussed. All this serves as an introduction to the 
sophisticated point target phase-return model of Chapter 4 , which accurately models the effects wavefront curvature for all imaging scenarios and squint angles. With this model, an efficient, image-domain space-variant post-filter will be designed, thereby lifting scene size restrictions when wavefront curvature is present in polar-formatted imagery.

\subsection{The Planar Wavefront Assumption - A Limi- tation of the Tomographic Paradigm}

Certain limitations of the tomographic paradigm, such as failing to predict the rangeoriented layover effects of three-dimensional targets into two-dimensional space, have been carefully addressed by $3 \mathrm{D}$ extensions to the tomographic model [6]. Perhaps the most significant limitation to this model, and ironically, the most recent to be addressed (this dissertation being the first to propose a complete solution), is based on the assumption of planar wavefronts in the construction of the projection functions of the scene reflectivity function. See Figure 3.1 for a comparison of the illuminated scene with realistic spherical wavefronts, as shown in part (a), to that of the planar wavefront assumption in part (b). This assumption leads to an annular-shaped geometric distortion of the formed imagery, as well as a more problematic spacevariant defocus (blurring) of the imaged scene. Mentioned by both Walker and Munson, the potential effects of this assumption on the quality of SAR imagery were noted, yet no solution was proposed, beyond restricting the imaging scenarios to those which closely followed the assumption. While the geometric distortions could be compensated through a geometric rewarping process after image formation, the scene size restriction was suggested in order to minimize the effects of the spacevariant defocus.

The polar-format algorithm can be described in terms of a tomographic process, and it is this analogy that lends explanation to the simplicity of the polar-format al- 
gorithm. Unfortunately, as a tomographic process, PFA-processed imagery degrades in quality (in terms of space-variant defocus) when the planar wavefront assumption no longer holds. As radars achieve ever increasing resolutions, this assumption breaks down, particularly at lower center frequencies and when imaging large patches at close range. Consequently, the planar wave assumption is not valid under these scenarios, yet it is no longer reasonable to restrict radars to collection scenarios for which the assumption is valid. When the assumption is violated, the deleritous effects on PFA-processed imagery are often referred to as range curvature or wavefront curvature effects. However, to be precise, range curvature is the range-oriented motion of scene targets, relative to the radar platform, as the aperture is being flown (see Figure 1.4). When this motion exceeds the distance represented by a single range bin in the formed imagery, then defocus occurs.

It is important to note that the polar-format algorithm compensates this range migration motion via the acquisition of samples on a polar grid, additional mocomp to the scene center if necessary, and the 2D interpolation of the phase history onto a Cartesian grid prior to inverse Fourier transformation. Otherwise, scene size must be severely restricted, as discussed in the previous chapter. What remains after this range migration compensation is actually residual, differential range curvature, which is based on the faulty assumption of planar EM wavefronts being transmitted by the antenna. This is specifically what is meant by the term wavefront curvature. While other image formation methods inherently compensate for wavefront curvature, this dissertation proposes a method for compensation in polar-formatted imagery, thereby extending the utility (with little additional computational cost) of the proven, straightforward polar-format algorithm, to produce high quality imagery in the presence of wavefront curvature. Furthermore, as will be shown in Chapter 6, polar-formatting is typically no more computationally burdensome (and for certain scenarios far more efficient) than other popular image formation methods. 


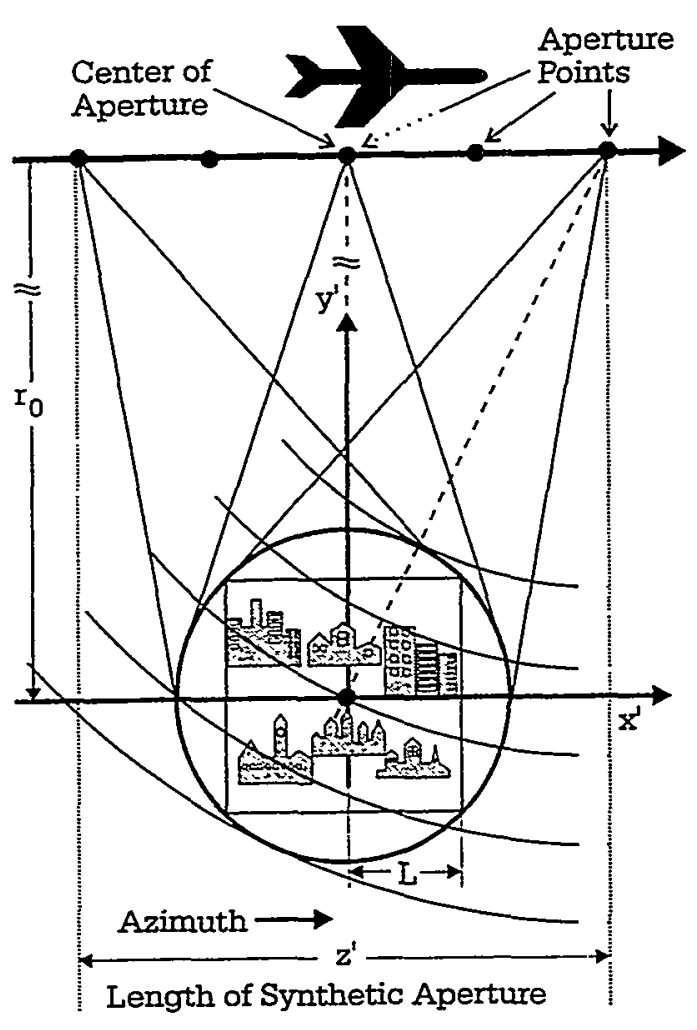

Spherical Wavefronts Actually Present

(a)

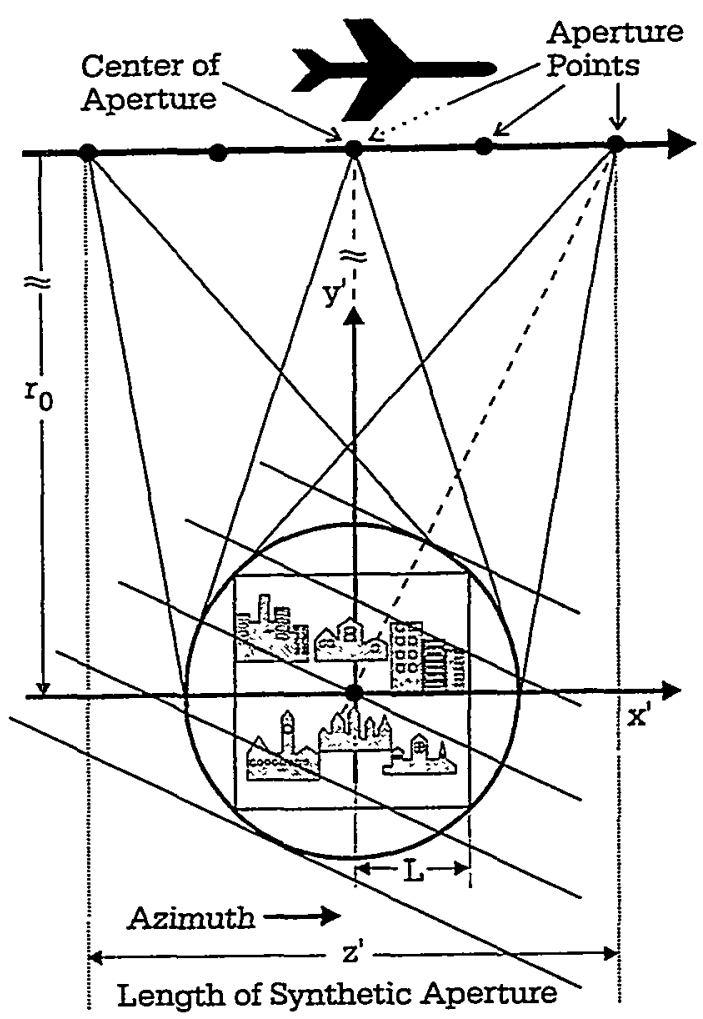

Planar Wavefronts that are Assumed (b)

Figure 3.1: Actual Spherical Wavefront Curvature Versus the Planar Assumption for Spotlight-Mode SAR

\subsection{The Cost of Ignoring Spherical Wavefronts}

This section describes the effects of wavefront curvature on polar-formatted spotlightmode SAR imagery. The wavefront curvature effects that appear in reconstructed imagery can be discovered by deriving an exact expression for the radar return signal as dictated by the actual spatial geometry (incorporating spherical wavefronts) and comparing this result with that obtained by the sampling of the Fourier domain as per the tomographic model. This derivation involves a single point target in the scene, with the realization that any scene may be considered to be the superposition of a large number of point targets. Ultimately, it will be revealed precisely how the assumption of spherical wavefronts renders invalid the relationship between 
the phase history and image domain reconstruction, as based on the tomographic paradigm. Furthermore, via this model, which will be presented in detail in Chapter 4 , it will be shown that wavefront curvature leads to both a geometric distortion of the reconstructed scene, as well as a space-variant defocus which can be severe in some imaging scenarios.

\subsubsection{Geometric Distortion}

It is instructive to describe the geometric distortion effects using a more intuitive model than that which will be presented in Chapter 4, even though it is incomplete in terms of describing quadratic defocus. This model will serve as the introduction to (and later it will be shown that the results are in agreement with) the sophisticated phase model of Chapter 4. This simple model will now be presented, as will illustrative examples of geometric distortion and defocus effects on real SAR imagery.

A simple slant plane model depicting the curved EM wavefronts is shown in Figure 3.2 , for a broadside collection. This model depicts the wavefront as it crosses the scene center, as transmitted (emanating) from mid-aperture. At first, it appears an incomplete wavefront curvature model for predicting the overall geometric distortion across the entire extent of the aperture. However, the MTRC compensation, which is based on the polar-coordinate acquisition of the phase history and any additional motion compensation to the scene center, ensures that the coherent integration of all Fourier slices over the aperture is represented by the single wave depicted. That is, the scene center serves as the phase center for the imaged scene, and the wave depicted through this point is sufficient to represent the distortion behavior across the scene.

For the slant plane model represented by Figure 3.2, let $L$ be the radius at range $y^{\prime}=0$ of the imaged scene, and $\theta_{L}$ be the angle formed at the platform by the edges extending to the scene center at $\left(x^{\prime}, y^{\prime}\right)=(0,0)$ and the scene edge at $\left(x^{\prime}, y^{\prime}\right)=(L, 0)$. 


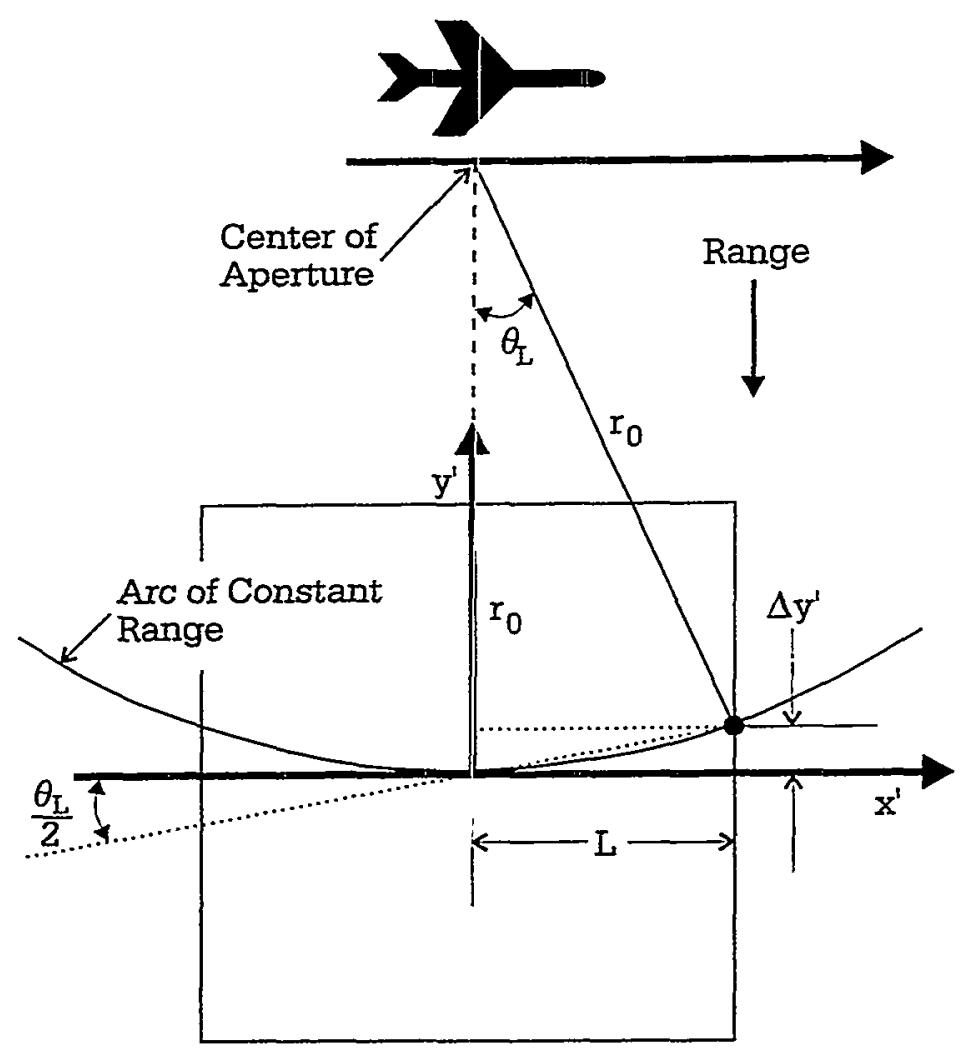

Figure 3.2: Broadside Model for Derivation of Geometric Distortion When Neglecting Spherical Wavefront Characteristics

Furthermore, $r_{0}$ is the radius of the arc that defines the curvature of the wave through both the scene center and patch edge. The difference in range between the actual curved wavefront and the planar assumption is $\Delta y^{\prime}$ at the scene edge. The value $\Delta y^{\prime}$ defines the geometric distortion, or sag in the range direction at the edge of the scene. The range sag is defined as follows:

$$
\Delta y^{\prime} \approx r_{0} \theta_{L} \sin \left(\frac{\theta_{L}}{2}\right),
$$

where the approximation $r_{0} \gg L$ is applied. Furthermore, when this approximation holds, $\theta_{L}$ is very small and consequently $\sin \left(\theta_{L} / 2\right) \approx \theta_{L}$, so that Equation (3.1) becomes

$$
\Delta y^{\prime} \approx \frac{r_{0}}{2} \theta_{L}^{2} \approx \frac{r_{0}}{2} \frac{L^{2}}{r_{0}{ }^{2}}=\frac{L^{2}}{2 r_{0}} .
$$


Thus, the range sag at the patch edge is a function of the slant plane standoff range (slant range) $r_{0}$, as well as the patch radius $L$. Interestingly, it is not a function of radar center frequency, bandwidth or image resolution, but instead strictly based on the degree of physical curvature of the transmitted EM pulse where it reaches the scene. The effect of this sag, in terms of geometric image distortion, is shown in Figure 3.3. Ironically, this approximation is least accurate when the patch is large or the slant range is short, which are precisely the conditions leading to large range sag. However, the approximation is accurate for many imaging scenarios, and gives an intuitive feel for the way in which the processed image is geometrically distorted when planar wavefronts are assumed, but in which the curvature is actually significant. As will be shown in Chapter 4, via the more complete phase model presented there, the geometric sag in range for any point (not just at the patch edge) is based on the linear phase error terms derived from that model. Specifically, a point in the slant plane image at some point $x_{0}^{\prime}$ will actually end up being displaced in range an amount of

$$
\Delta y^{\prime}=\frac{x_{0}^{\prime 2}}{2 r_{0}}
$$

when significant wavefront curvature is present. Again this equation assumes $r_{0} \gg L$. When the point is at the patch edge, that is, $x_{0}^{\prime}=L$, Equations (3.2) and (3.3) are the same. Similarly, the same point will be displaced in azimuth from $y_{0}^{\prime}$ by

$$
\Delta x^{\prime}=\frac{x_{0}^{\prime} y_{0}^{\prime}}{r_{0}}
$$

given the same assumption of a large ratio between slant range and patch radius. As will be shown in the phase model of Chapter 4, these position translations are a direct result of the Fourier transform shift property, which states that linear phase terms in the Fourier domain are represented as translations in the processed image domain. Of course, linear translation is necessary to properly place the target in the proper position in the processed image. However, the phase terms and translations of Equations (3.3) and (3.4) specify residual terms representing wavefront curvature errors that are in addition to those for proper placement of the target in the imaged 
scene. It is these terms (in addition to the quadratic terms to be described next) that render invalid the inverse Fourier transformation of the polar-formatted data into a image-domain scene. Consequently, these linear phase terms, based on the invalid planar wavefront assumption, induce translations of the reconstructed points in both dimensions of the image domain, resulting in a position-dependent shifts that distort a square image patch into an annulus. These effects are depicted in Figure 3.3. As expected, the amount of distortion prescribed by the linear phase terms ends up placing a point in the reconstructed image along the actual circular arc of the wavefront, as opposed to the straight line assumed to be representing the wavefront. As the planar wavefront assumption becomes increasingly inaccurate, as prescribed by an increase in patch radius or decrease in slant range, the resulting geometric distortion becomes more severe. When comparing the orientation of the spherical wavefront of Figure 3.2 to that of the corresponding image distortion in Figure 3.3, one notes that they are in opposite directions. This is because the curved wavefront "falls short" of a point in the scene (displaced from the center) compared to the planar wravefront prediction. Consequently, the point seems farther than is actually the case, and the imagery formed under the planar wavefront assumption distorts as to bend away from the radar platform's aperture center. The model of Chapter 4 is much more complete in that it gives an exact description of geometric distortion at all ranges and patch radii, for both squinted and broadside collections.

Figure 3.4 illustrates the effects of wavefront curvature on actual spotlight-mode SAR imagery. It is important to note that while the imagery is real, the geometric distortion has been induced by artificially applying severe linear phase errors to the formed imagery; more severe than was actually present in the imagery at the time of collection. This serves to illustrate the geometric distortion that would actually have been present had this been a much larger scene, or taken at much closer range than was actually the case. This distortion is based on predicted translations of Equations (3.2) and (3.3), yet for the unrealistically close slant range of a few hundred meters, thereby exaggerating the distortion effects. The lower portions of Figure 3.4 


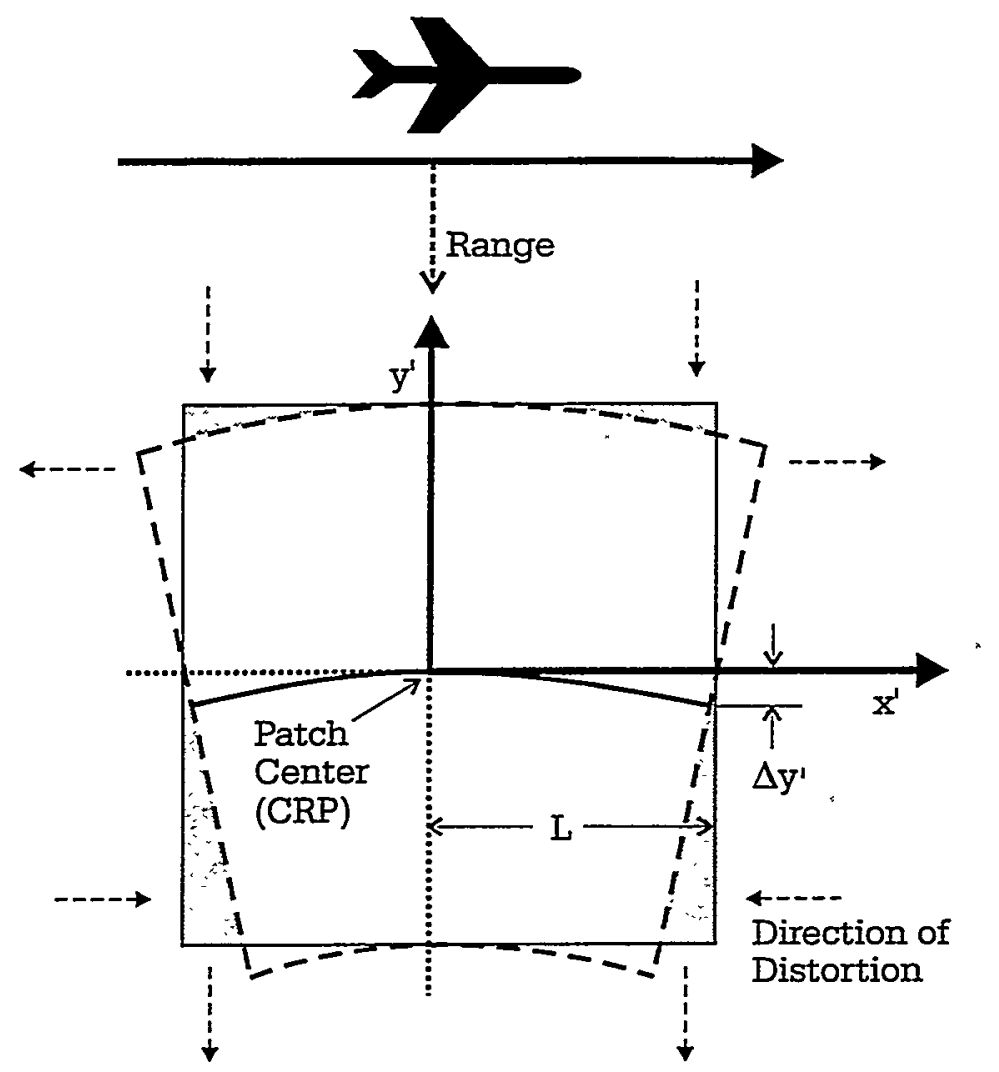

Figure 3.3: Geometric Image Distortion From Wavefront Curvature - Broadside Collection

(parts (c) and (d)) represent enlarged views of the pentagon scene, before and after geometric correction, respectively. The geometric sag is evident in part (c), where the straight edges of the building are noticeably distorted, and corrected in part (d) via a post-warping procedure.

While Figure 3.4 serves as an illustrative example with an exaggerated case of geometric distortion, this phenomenon is indeed a serious issue in real imagery. Take for example, the imagery presented in Figure 3.5. The geometric distortion present in this image is that which has occurred naturally; as a consequence of neglecting the effects spherical wavefronts during polar-format processing. This image is of a test track area at Sandia National Labs in Albuquerque, New Mexico. The rails of this sled track are made to be very straight, as rocket powered test sleds moving 


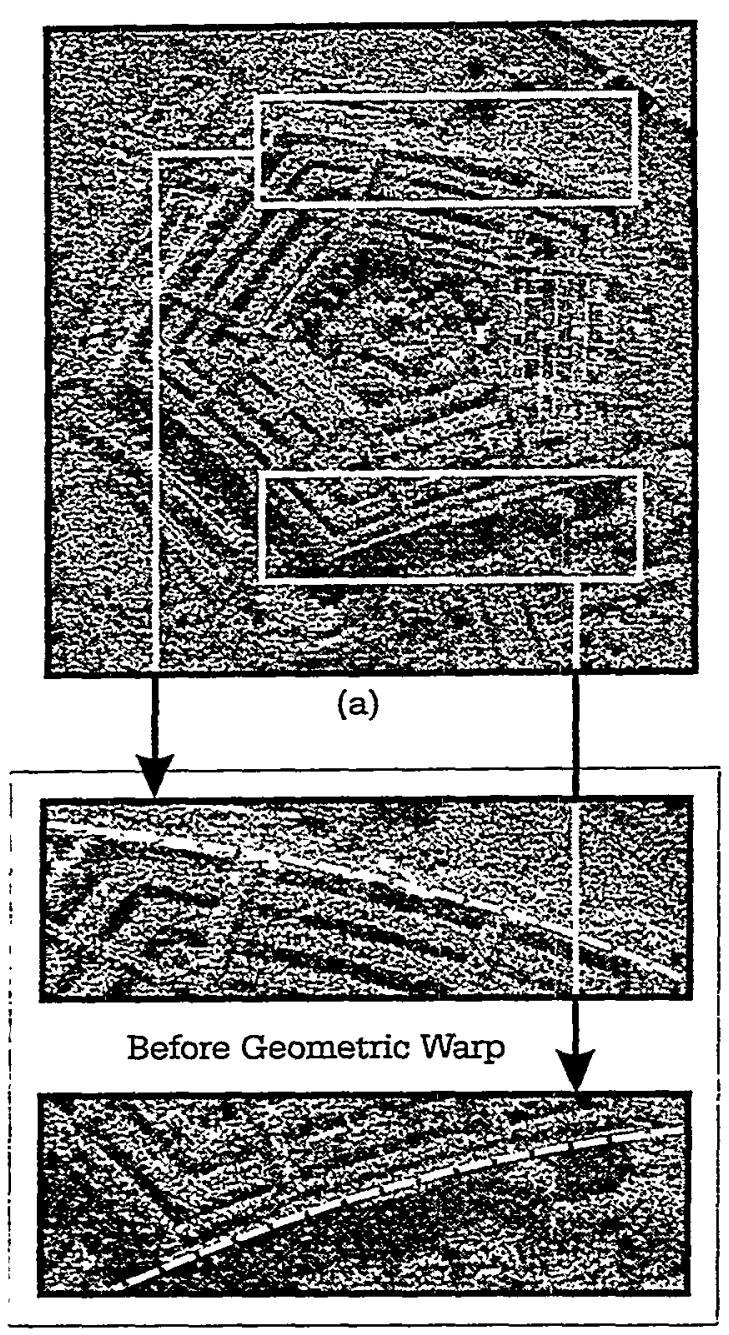

(c)

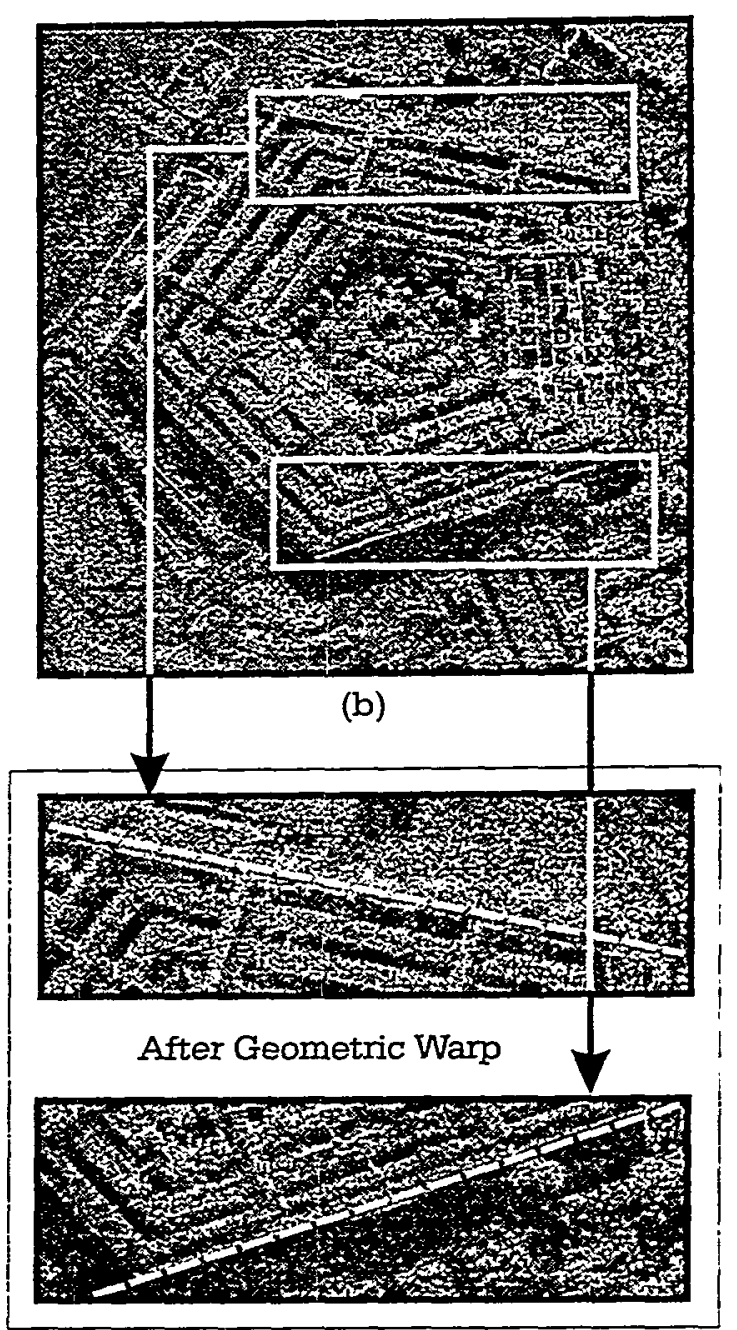

(d)

Figure 3.4: Simulated Geometric Distortion Effects of Wavefront Curvature

at extremely high velocities are launched down the rails. This sled track is ideal in demonstrating the effects of wavefront curvature since it is known to be a long, straight object and also a very good radar reflector. The specific imaging parameters for this scene are as follows: The nominal standoff range $r_{0}$ is 6000 meters, the range and cross-range resolutions $\rho_{y}$ and $\rho_{x}$ are both 1 foot ( 0.33 meters), and the radius of the patch from scene center $L$ is 175 meters. This scene was imaged from Sandia's high resolution testbed spotlight-mode SAR [41] at $\mathrm{Ku}$ band (14-16 $\mathrm{GHz}$ ), and processed using the polar-format algorithm. Interestingly, for such a 


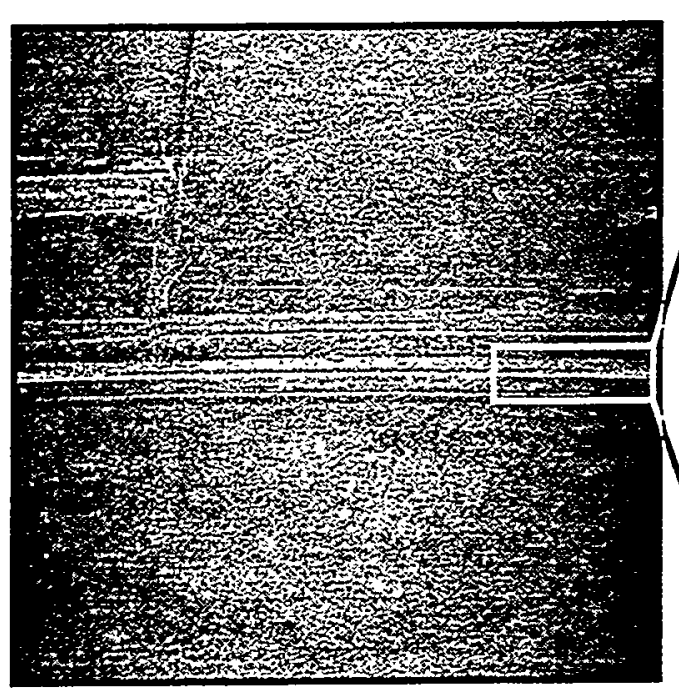

(a)

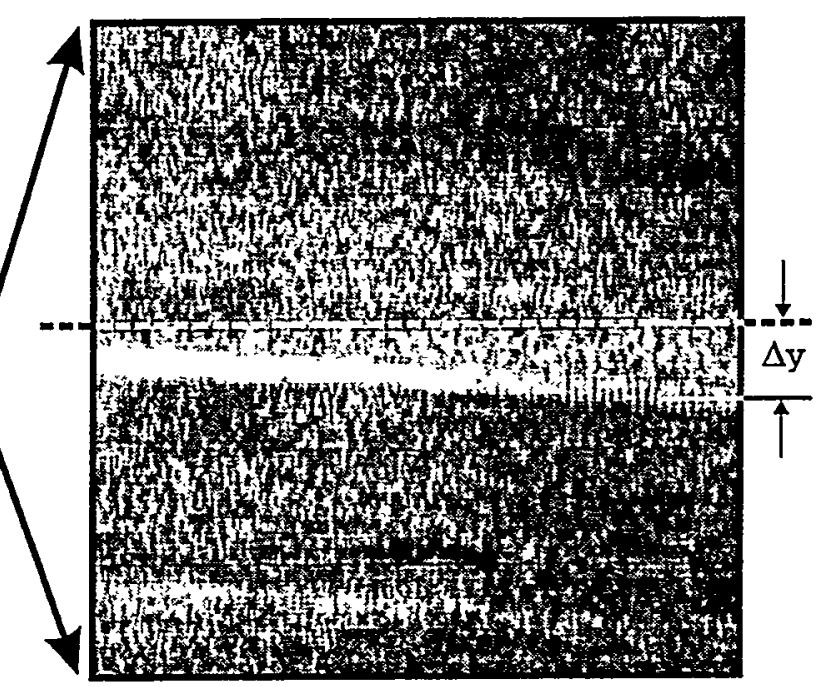

(b)

Figure 3.5: Actual Ku-Band SAR Image of Sled Track With Wavefront Curvature Distortion (1 Ft. Res.)

\begin{tabular}{||c|c|}
\hline \multicolumn{2}{||}{ Ku Band SAR, $f_{0}=14-16 \mathrm{GHz}, r_{0}=6000 \mathrm{~m}$} \\
\hline Patch Radius, $L(\mathrm{~m})$ & Range Sag, $\Delta y^{\prime}(\mathrm{m})$ \\
\hline \hline 100 & 0.83 \\
200 & 3.33 \\
300 & 7.50 \\
400 & 13.33 \\
500 & 20.83 \\
600 & 30.00 \\
\hline
\end{tabular}

Table 3.1: Range "Sag" at Scene Edge for Ku Band Sled Track Example

small scene radius $(L=175 \mathrm{~m})$, the observed geometric sag at the patch edge is quite significant. Based on Equation (3.2) for $L=175$ and $r_{0}=6000$, the predicted sag is $L^{2} / 2 r_{0}=2.55$ meters. This corresponds to approximately 8 range resolution cells, given the range resolution of $\rho_{y}=0.33$ meters and assuming no additional oversampling (zero padding) during Fourier inversion. For this example, the range sag at the scene edge for various patch radii is given in Table 3.1.

The geometric distortion is easily removed by post-warping the reconstructed image. Since the amount of translation for each point can be predicted by the linear 
phase terms of Equations (3.3) and (3.4), each point can be correctly restored to its "proper" location. A second-order polynomial can be generated from these equations to describe the geometrically correct placement for any point in the distorted scene. It is important to note that these equations predict the amount of linear displacement that occurs in the distortion of a scene to its "improper" place in the warped image. Geometric correction requires the inverse operation; that is, the movement from a point from a position in the distorted image to that which is geometrically correct. Consequently, the polynomial warping function must represent the inverse of the translations specified by Equations (3.3) and (3.4). This is discussed in great detail in Chapter 5. Once the proper positions of the points have been determined, an interpolation of the image domain values using a simple bilinear interpolator is all that is necessary to correct the geometric distortion. This is in contrast to the two-dimensional polar-to-rectangular reformatting of the phase history data, which requires a more complicated sinc (or equivalent) interpolation scheme.

This dissertation presents geometric distortion as a prominent consequence of ignoring wavefront curvature effects in PFA processed, spotlight-mode SAR imagery. The model of Figure 3.2 was presented to explain the nature of the geometric distortion, and was used to derive a relation for predicting range sag in terms of patch radius and slant range. Furthermore, as will be shown in Chapter 4, the robust model representing the phase return from a point target will describe this geometric distortion in terms of linear phase errors, which render invalid the tomographic relationship between the Fourier phase history and the imaged scene. However, the geometric distortion, prominent in many SAR imaging scenarios, has been dealt with since the inception of the PFA algorithm. Specifically, the necessary post-warping of the image under these scenarios has always been incorporated as a routine step in the polar-format algorithm processing sequence. This discussion, and that of Chapter 4 , provides valuable insight as to the nature of this distortion. However, the unique contribution of this work is the characterization and compensation of the problematic space-variant defocus that appears when severe wavefront curvature is present, 
and not the geometric distortion.

\subsubsection{Space-Variant Defocus}

Wavefront curvature defocus effects occur in certain spotlight-mode collection modes that include imaging at close range or using low center frequencies, especially when imaging large ground patches. These defocus effects are particularly troublesome in that they effectively lower scene resolution (and greatly hinder interpretability), while their space-variant nature makes them difficult to compensate. They occur in conjunction with the geometric distortion effects described previously, yet are less visibly prominent when wavefront curvature is minimal. In fact, as shown in Figure 3.5, geometric sag is evident but there is no visible image blurring. However, given other specific imaging scenarios in which wavefront curvature becomes more severe, the corresponding increase in defocus causes significant visual degradation to processed imagery.

In contrast to the induced geometric distortion, the defocus effects can not be removed via a post warping procedure. Instead, it will be shown that a one-dimensional space-variant convolution (deblurring) filter, applied as a post-processing step to the image domain data, can adequately compensate the defocus effects induced by wavefront curvature. As will be derived in detail in Chapter 4, these blurring effects are mostly due to quadratic phase error terms present in the specific scenarios for which the planar wavefront assumption does not hold. The magnitude of this defocus effect is a function of the range and cross-range position of the target, and becomes greater for those targets placed further from the scene center. For the simplest case, a broadside collection whereby the slant range is much greater the patch radius; that is, assuming $r_{0} \gg L$, the quadratic, Fourier domain phase error is found to be

$$
\phi_{2 t}=H\left(X^{\prime}\right) \approx \frac{x_{0}^{\prime 2}-y_{0}^{\prime 2}}{2 r_{0} k_{0}} X^{\prime 2}
$$

where $k_{0}=4 \pi / \lambda$ is the nominal annular radius in the Fourier domain, $r_{0}$ is the slant 
range of the radar platform, $\left(x_{0}^{\prime}, y_{0}^{\prime}\right)$ is the location of a target in the reconstructed slant-plane image, and $X^{\prime}$ is the phase-history (Fourier domain) frequency extent associated with the cross-range dimension. The $X^{\prime}$ term appears squared and this indicates the phase error $\phi_{2 t}$ is indeed quadratic in nature, and the relation is also represented as $H\left(X^{\prime}\right)$, indicating it is a Fourier domain blurring filter as well. Note that there is no $Y^{\prime}$ term in the relation, and therefore, no range frequency-dependent blurring. That is, the filter is one-dimensional in the cross-range direction only. This observation will be confirmed, as will the validity of Equation (3.5), via the robust phase model for a point target to be presented in Chapter 4 .

The effect of the quadratic phase term in the phase history is to convolve the image with a kernel consisting of the Fourier transform of a complex exponential having that quadratic phase. Consequently, the image will be blurred (in the crossrange direction given the approximation in this case) by an amount commensurate with the width of the convolving kernel, which in turn depends on the peak amplitude of the quadratic. The actual amount of image defocus that occurs in the processed imagery, as a function of peak quadratic phase error, is derived in Appendix A, and its application is carefully discussed in Chapters 4 and 5 .

The amount of quadratic defocus is spatially variant, as indicated by Equation (3.5). In particular, along the pair of diagonal lines in the image plane given by $y^{\prime}=\left|x^{\prime}\right|$ (assuming the point has been correctly rewarped to eliminate geometric distortion), the amount of defocus is exactly zero. The defocus is maximal at the image locations $\left(x^{\prime}, y^{\prime}\right)=(0, L)$ and $\left(x^{\prime}, y^{\prime}\right)=(L, 0)$. Also, this relation reveals that the quadratic phase error increases with decreasing slant range. as indicated by $r_{0}$ in the denominator, and with decreasing radar frequency, as indicated by the correspondingly increasing $\lambda$ in the denominator of $k_{0}$. These quadratic phase errors are not represented in the simple spherical curvature model of Figure 3.2, yet will be quantified in the point target phase return model of the next chapter. In essence, Equation (3.5) represents the Fourier transform of the space-variant blur function (ie: the transform of the blurring convolution kernel), and is shown to be phase-only 
(without regard to magnitude), and one-dimensional in the cross-range direction.

Without yet delving into implementation details, it is still reasonable to assume (for now, if only intuitively) that the conjugate multiplication of this blur function with the appropriate Fourier data will result in the deconvolution (refocus) of a portion of the imaged scene when those data are inverse Fourier transformed. Indeed, by spatially varying this deconvolution filter as appropriate, it will be shown in the following chapters that the image can be efficiently post-filtered to compensate the defocus effects of wavefront curvature. As with the previously presented pentagon illustrations for geometric distortion, examples will now be presented that simulate the defocus effects as prescribed by Equation (3.5). That is, a space-variant convolution kernel, as specified by this equation, has been applied to focused imagery and shown in Figures 3.6 and 3.7. These illustrations demonstrate the space-variant defocus associated with the faulty assumption of planar wavefronts. As with the previous geometric distortion example, the amount of defocus exhibited in these figures is greatly exaggerated for illustrative purposes. However, the space-variant behavior is exactly which is found in real imagery exhibiting this defocus, though of greater magnitude in this example. In particular, parts (c) and (d) of Figure 3.6 are expanded views, before and after "correction," of defocus in a severely affected region. In reality, the corrected version is simply that which does not have the phase error of Equation (3.5) applied to it. That is, it has not actually been post-filtered for wavefront curvature compensation (though it could be). In this particular illustration, the expanded views are of a point in the region of $\left(x^{\prime}, y^{\prime}\right)=(L, 0)$, where defocus is stated to be most severe. In contrast, the expanded views of Figure 3.7 are in the neighborhood of $y^{\prime}=\left|x^{\prime}\right|$, where defocus is said to be minimal. These two examples illustrate the space-variant nature of the defocus induced by the faulty assumption of planar wavefronts illuminating the scene.

It must be emphasized that this space-variant defocus phenomenon is not restricted to artificial dissertation examples, and it is not a theoretical behavior to be found only in mathematical analyses. In fact, defocus effects due to faulty pla- 
nar wavefront assumptions occur in a number of real SAR imaging systems under certain scenarios. Of particular interest are the ultra-wideband (UWB) SAR and L-Band SAR, as described in [12, pp. 435-439]. These radars are known for their foliage penetrating ability $[81,82,83]$, which is a phenomenon associated with their relatively low center frequencies. The large imaged patch sizes associated with these radars, in conjunction with the low center frequencies and close-in slant ranges, clearly render the planar wavefront assumption invalid. Consequently, these particular SARs generate imagery that suffers from significant space-variant defocus when their polar-formatted phase histories are processed via the polar-format algorithm. Furthermore, particularly in the case of the L-Band collection, the patch diameter is on the order of the slant range distance, rendering invalid the approximation $r_{0} \gg L$, on which Equation (3.5) is based. Fortunately, the robust phase model to be presented in Chapter 4 has no reliance on this approximation. As a verification of this model, Chapter 5 will present synthetically generated spotlight-mode SAR imagery; based on the radar parameters of the UWB and L-Band SAR, and exhibiting the wavefront curvature defocus effects exactly as are produced with the actual SAR systems. The performance of the new space-variant post-filter will be considered for these practical examples via the synthetic target generator, which is void of all other phase error effects except those associated with wavefront curvature.

Of course, while fine for illustrative purposes, the approach of artificially applying (and removing) quadratic phase errors to the imagery of Figures 3.6 and 3.7 begs the question as to whether Equation (3.5) is correct. Clearly, if applying a faulty defocus convolution filter, the conjugate multiplication in the Fourier domain by the transform of this same filter will correctly remove the induced blur, even if the filter is not an appropriate representation of the space-variant defocus induced by wavefront curvature. However, the reader can rest assured in knowing that the defocus phase error terms will soon be derived using a realistic model for the phase return of a point target, and confirmed by analyzing the performance of the spacevariant post-filter which is based on this model, by way of synthetic target imagery 


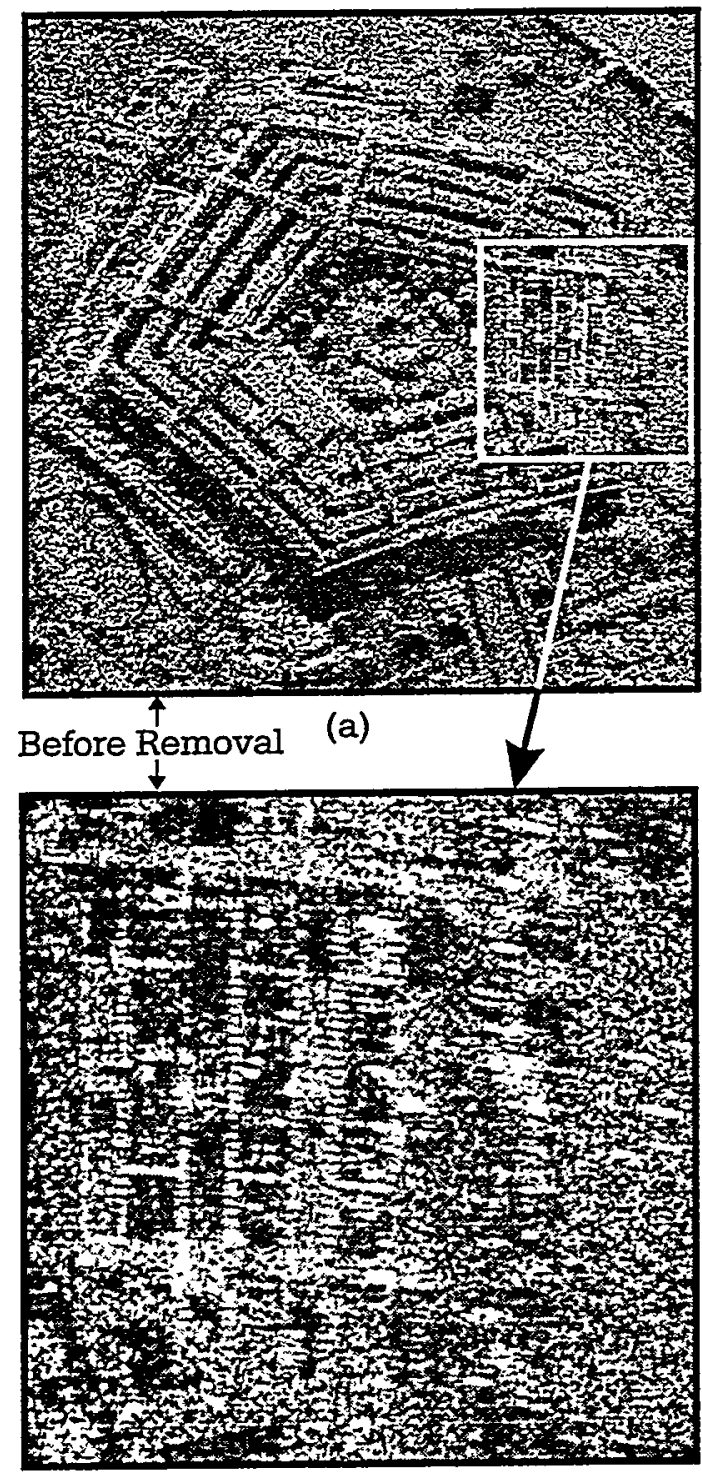

(c)

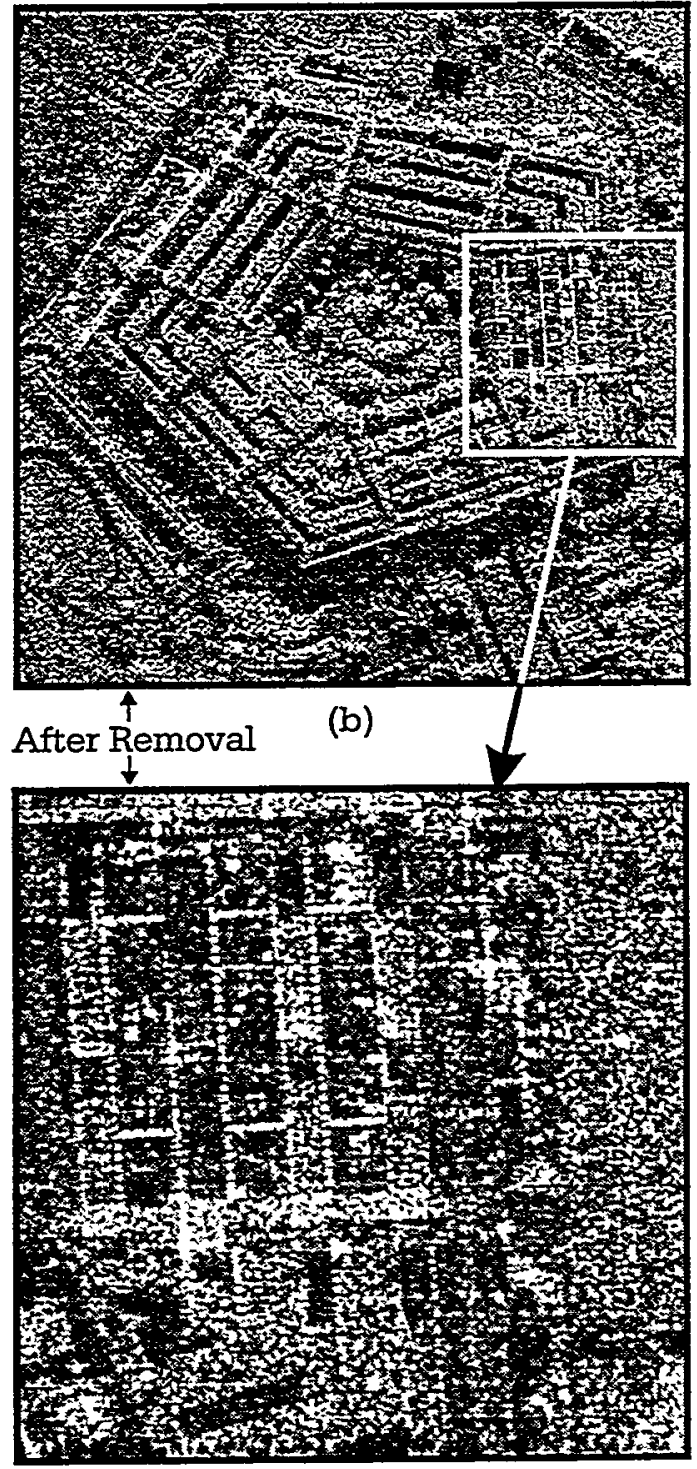

(d)

Figure 3.6: Simulated Wavefront Curvature Effects Before and After Removal Severely Defocused Region

that has been generated via the tomographic model for the ideal phase return from a point scatterer. In essence, synthetic imagery will be generated which, by the very nature of the tomographic paradigm, includes the extra phase terms which induce distortion and space-variant defocus in the processed imagery. For the practical, real-world scenarios to be presented, these phase error terms are particularly large. 
Chapter 3. The Planar Wavefront Assumption in Polar-Formatted Imagery

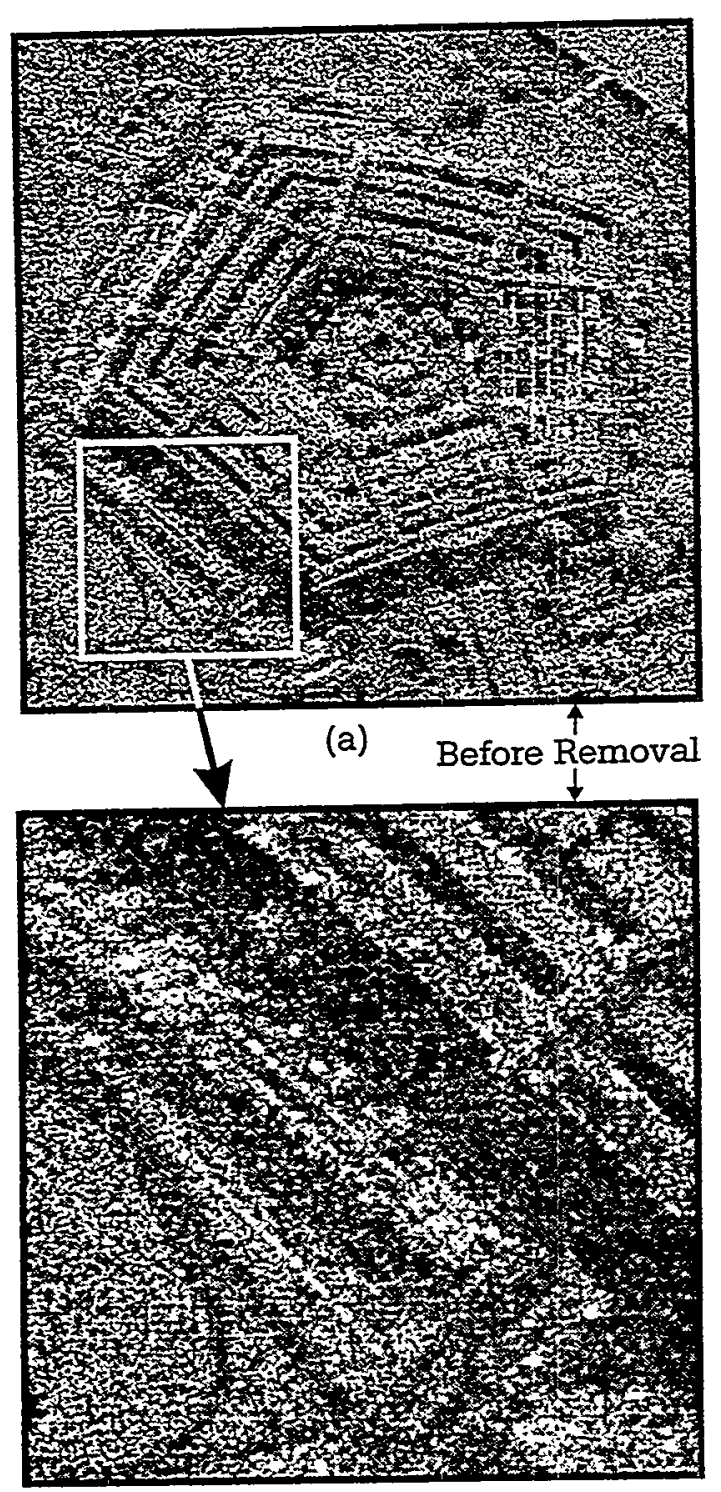

(c)
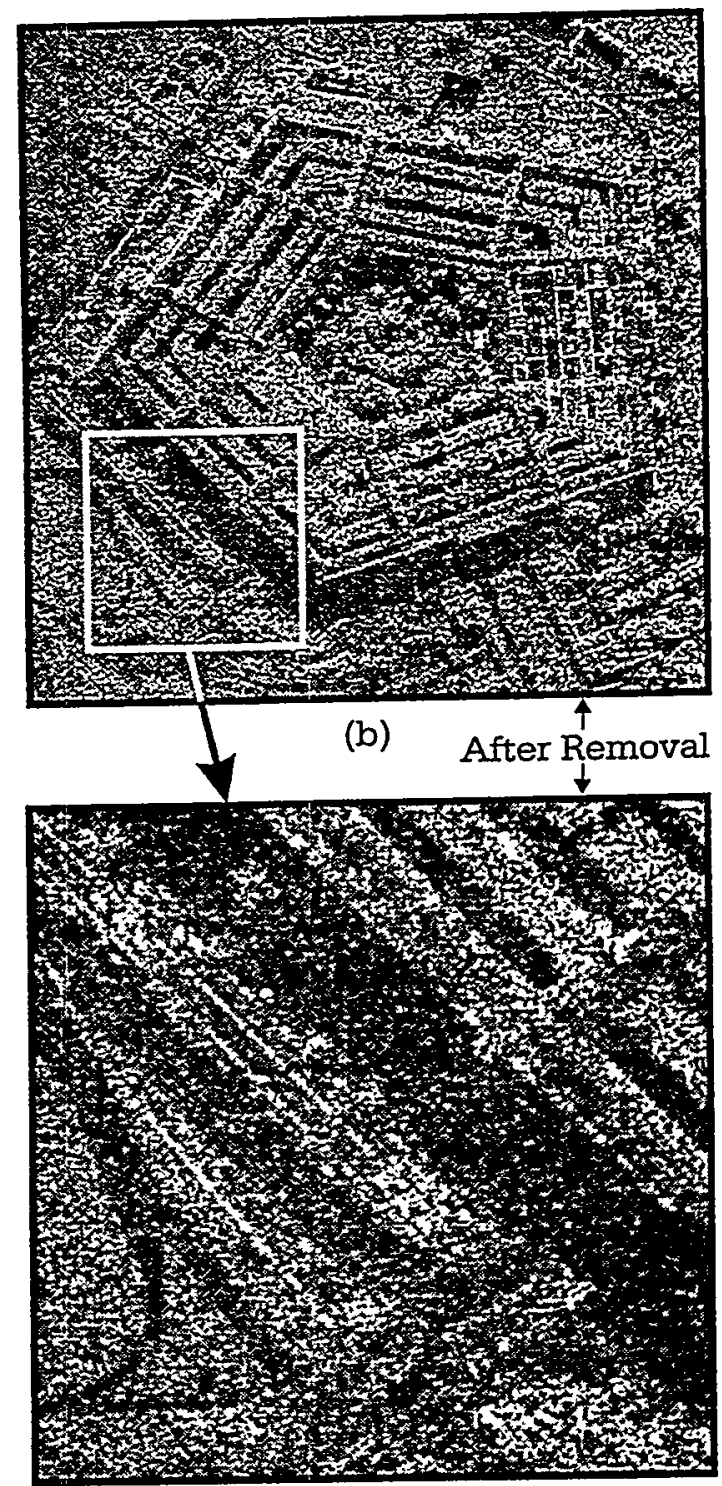

(d)

Figure 3.7: Simulated Wavefront Curvature Effects Before and After Removal Slightly Defocused Region

The imagery will then be refocused via the space-variant post filter derived from the phase return model, by compensating the terms that render invalid the tomographic relationship between the Fourier phase history data and the image domain. 


\subsection{Scene Size Limitations Due to Wavefront Cur- vature Defocus Effects}

The faulty assumption of planar wavefronts in certain imaging scenarios leads to linear, quadratic, and higher order phase error terms that negatively affect the polarformat processed imagery. The linear phase terms lead to a geometric distortion that is easily corrected via a post warping procedure. The quadratic phase term, as described by Equation (3.5), induces a position-dependent (space-variant) defocus that degrades the image in the cross-range direction. The amount of quadratic phase (and the defocus induced as a consequence) becomes increasingly severe at the edges of large patches near the axes, and subsides along the diagonals of the scene. Furthermore, the defocus increases as the slant-range or the radar center frequency decrease, or as the cross-range resolution increases. The quadratic defocus effects are less prominent than the geometric distortion for a given scenario, as will be further explored in the sled track defocus analysis of this section. However, there also exist cubic and higher order terms that serve to further defocus and degrade the imagery in the presence of significant wavefront curvature.

Fortunately, as in the case where the quadratic phase term is small compared to the linear range sag term, the cubic phase errors (and their associated defocus effects) are significantly less prominent than the quadratic phase error. This is due to the inverse factorial scaling of each subsequent phase error (with respect to polynomial order), when the phase return from a point target is represented by the Taylor series decomposition. This mathematical analysis is given in the next chapter. Regardless, in practice, the visual image degradation from these higher order terms (larger than second order) is negligible and can be ignored. In chapter 6 , the residual, higher order phase errors for the L-Band SAR example will be plotted and shown to be negligible.

The plots of Figure 3.8 show the cross-range defocus effects for quadratic phase 


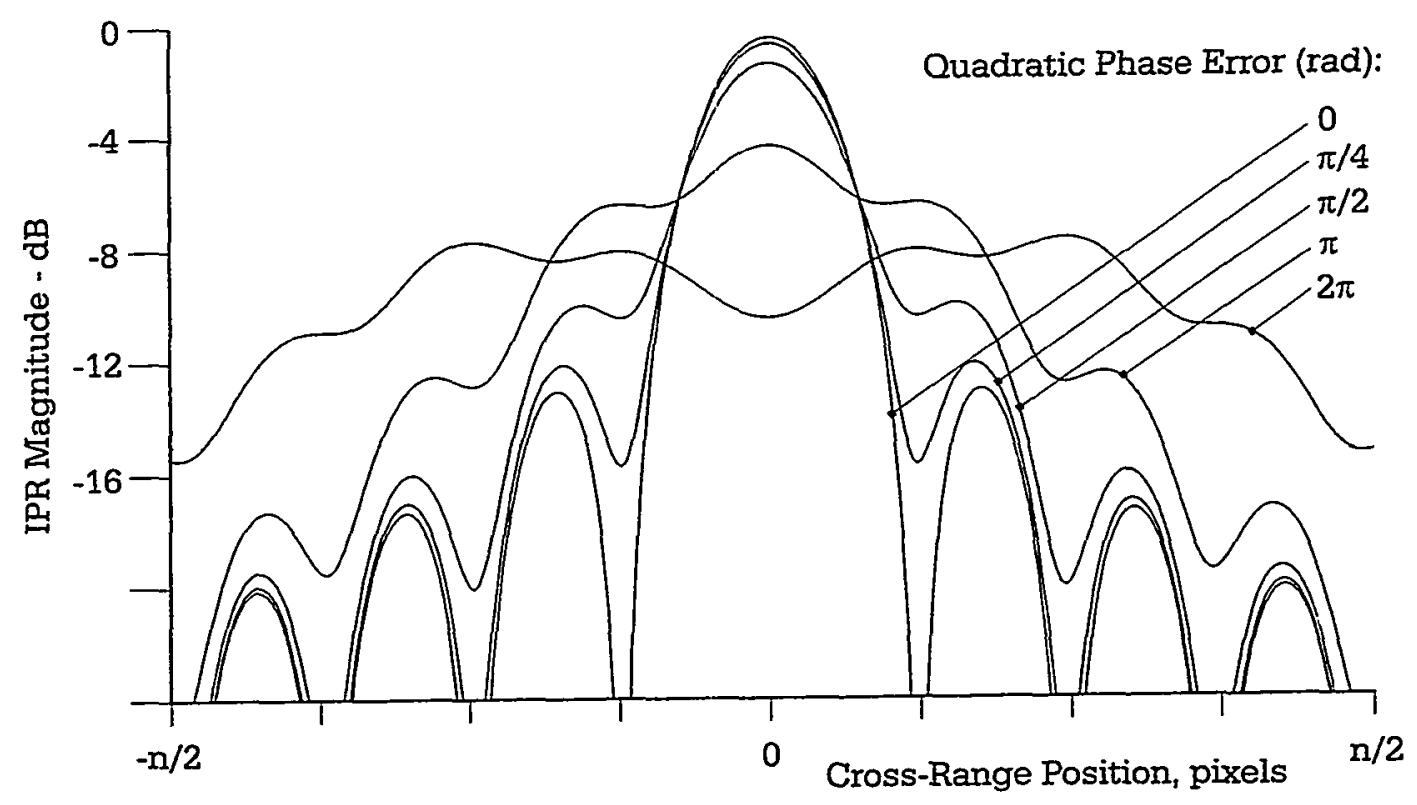

Figure 3.8: Cross-Range Defocus for Various Values of Peak Quadratic Phase

errors of varying amplitudes (peak quadratic phase). The plot corresponding to a zero-amplitude phase error is the ideal point-target response function, or impulse response function (IPR). Quadratic phase errors of various peak amplitudes have been applied to this complex function (in the Fourier domain). After inverse Fourier transformation, the magnitude responses of the various functions are as shown. The data has been properly zero-shifted, via alternate row/column multiplex (or proper linear phase ramp multiplication) so that the DC (zero) component is in the center of the plot. It is evident from these plots that a quadratic phase error of sufficient amplitude can significantly degrade image quality. As a rule of thumb, the spacevariant defocus associated with a quadratic phase error of $\pi / 4$ radian or less is considered to be negligible.

The value of $\pi / 4$ is a somewhat objective limit on the peak magnitude of the quadratic phase error, and is based on the visual distortion of a point target. However, the development included in Appendix A ensures (from a mathematical perspective) that this phase error limit prevents visible broadening of the IPR. That 
is, by limiting the peak quadratic phase error to $\pi / 4$ radians, the defocus is kept to within subpixel levels and the visual smear remains negligible. Based on this peak limit, a relation can be derived that specifies the largest possible patch size allowable for this phase limit. This is accomplished by relating the phase error calculation of Equation 3.5 to the maximum allowable peak error for negligible target smearing, and solving for the maximum patch size:

$$
\left|\frac{x_{0}^{\prime 2}-y_{0}^{\prime 2}}{2 r_{0} k_{0}}\right| X_{1}^{\prime 2} \leq \frac{\pi}{4}
$$

where $X_{1}^{\prime}$ is the cross-range frequency extent defining the maximum extent of the aperture $\left(\left|X^{\prime}\right| \leq X_{1}^{\prime}\right)$, and $-\frac{\Delta X^{\prime}}{2} \leq X^{\prime} \leq \frac{\Delta X^{\prime}}{2}$, where $\Delta X^{\prime}$ is defined by Equation (2.17). The nominal cross-range resolution of the image formed from such an aperture is $\rho_{x^{\prime}}=\pi / X_{1}^{\prime}$, which is known as the half-power width of the ideal response function and is based on Equation (2.19). Furthermore, from Equation (2.14), $k_{0}=2 \omega_{0} / c=$ $4 \pi / \lambda$, and from Equation (3.5), recall that the defocus is maximal at the locations $\left(x^{\prime}, y^{\prime}\right)=(0, L)$ and $\left(x^{\prime}, y^{\prime}\right)=(L, 0)$. Consequently, the patch size restrictions are in terms of both range and cross-range radius limits and are found to be

$$
\begin{aligned}
& \frac{x_{0}^{\prime 2}}{2 r_{0} k_{0}} X_{1}^{\prime 2} \leq \frac{\pi}{4}, \\
& \frac{y_{0}^{\prime 2}}{2 r_{0} k_{0}} X_{1}^{\prime 2} \leq \frac{\pi}{4},
\end{aligned}
$$

and by substituting for $X_{1}^{\prime}$ and $k_{0}$, these restrictions can be expressed in terms of $\rho_{x^{\prime}}$ and $\lambda$ :

$$
\begin{aligned}
& x_{0}^{\prime} \leq \rho_{x^{\prime}} \sqrt{\frac{2 r_{0}}{\lambda}}, \\
& y_{0}^{\prime} \leq \rho_{x^{\prime}} \sqrt{\frac{2 r_{0}}{\lambda}} .
\end{aligned}
$$

Thus, the maximum image dimensions in both range $y_{0}^{\prime}$ and cross-range $x_{0}^{\prime}$, relative to the cross-range resolution $\rho_{x^{\prime}}$, must be restricted to maintain good focus. These restrictions are most severe at close range (small $r_{0}$ ) and at low frequencies (large $\lambda$ ), as implied by Equation (3.5), on which they are based. Also, note the patch size 
restrictions increase linearly as a function of increasing (ie: smaller valued) crossrange resolution. Recall from Equation (2.19) that the cross-range resolution $\rho_{x^{\prime}}$ can be represented in terms of the extent of the synthetic aperture $\Delta \theta$; specifically, $\rho_{x^{\prime}}=\lambda / 2 \Delta \theta$. Thus, the patch radius limits can be alternatively expressed in terms of the synthetic aperture extent. From Equation (3.8),

$$
\rho_{x^{\prime}} \sqrt{\frac{2 r_{0}}{\lambda}}=\sqrt{\rho_{x^{\prime}}^{2} \frac{2 r_{0}}{\lambda}}=\sqrt{\left(\frac{\lambda}{2 \Delta \theta}\right)^{2} \frac{2 r_{0}}{\lambda}}=\sqrt{\frac{\lambda r_{0}}{2(\Delta \theta)^{2}}}
$$

and by substitution back into Equation (3.8),

$$
\begin{aligned}
& x_{0}^{\prime} \leq \sqrt{\frac{\lambda r_{0}}{2(\Delta \theta)^{2}}}, \\
& y_{0}^{\prime} \leq \sqrt{\frac{\lambda r_{0}}{2(\Delta \theta)^{2}}} .
\end{aligned}
$$

As would be expected, an increase in aperture extent improves the cross-range resolution $\rho_{x^{\prime}}$ and consequently reduces the maximum allowable patch size. In the case of Equation (3.10), it appears that reducing radar center frequency $f_{0}$, thereby increasing wavelength $\lambda$, is actually relaxing the patch size requirement, which is contrary to what is implied by Equation (3.8). However, this is not the case, as one must realize that resolution is not held constant in the case of Equation (3.10) unless the aperture extent is increased in proportion with the wavelength. This results in the denominator outgrowing the numerator (because of the squared denominator term) and the maximum patch size shrinks, as expected, in the case where resolution is held constant.

These simple equations for deriving patch size limits, as well as Equations (3.3) and (3.5) which specify the respective geometric range sag and quadratic phase error, are powerful in their ability to predict the geometric distortion and space-variant defocus effects induced by wavefront curvature. This is true when the assumption is valid of a large slant-range to patch radius $\left(r_{0} \gg x_{0}^{\prime}\right)$. For example, recall that there was significant geometric distortion, yet no visible defocus in the sled track example of Figure 3.5. Assuming a center frequency $f_{0}$ of $14 \mathrm{GHz}$ (where $\lambda=c / f_{0}$ ) and a 
slant-range of $6000 \mathrm{~m}$, by the prediction of Equation (3.8), quadratic defocus for this scenario would not become noticeable until the scene radius exceeds $250 \mathrm{~m}$. Thus, one would not expect visible defocus in this example since the patch radius is only 175 $\mathrm{m}$, and indeed no defocus is apparent anywhere in the scene. It is interesting to note that the radius of the scene where defocus would just become evident; specifically, at a radius of $250 \mathrm{~m}$, the geometric sag in range would be over $5 \mathrm{~m}$ (or more than 15 range cells).

Clearly, in the presence of wavefront curvature, the amount of geometric sag is usually quite significant compared to the amount of space-variant defocus present in the scene. However, by no means does imply that defocus is not a significant problem in real imagery. Take for example, the UWB SAR mentioned in the previous section. This foliage penetrating SAR typically operates at a center frequency $f_{0}$ of $469.5 \mathrm{MHz}$ and a slant range $r_{0}$ of $6240 \mathrm{~m}$ [12, pp. 437-439]. Based on these parameters and a cross-range resolution of $0.66 \mathrm{~m}$, Equation (3.8) predicts a maximum allowable scene radius of only $93 \mathrm{~m}$, if defocus effects are to be avoided. However, scene sizes for this radar are typically $600 \mathrm{~m}$ in cross-range radius. At this maximum radius, Equation (3.5) predicts a quadratic phase error of $\phi_{2 t}=32.7$ radians, and consequently, Appendix A (Eqs. (A.17) or (A.28)) can be called upon to determine that resolution degrades at the scene edges by a factor of nearly 42 ! Thus, for this radar, the scene radius must limited to 15.5 percent of the desired size in order to avoid noticeable space-variant defocus. If this limit is ignored and instead the full $600 \mathrm{~m}$ patch is imaged, the degradation due to defocus is such that closely placed targets near the scene edges are indistinguishable. Indeed, space-variant defocus arising from the faulty assumption of planar wavefronts can be quite significant in certain scenarios of polar-formatted spotlight-mode SAR.

Table 3.2 lists scene size restrictions for some common SAR parameters, based on Equation (3.8). As is shown, in order to constrain the defocus to subpixel levels $\left(\phi_{2 t} \leq \frac{\pi}{4} \mathrm{rad}\right)$, the patch radius must be reduced as resolution increases or center frequency decreases, for a given fixed slant range. These patch size limits are based on 


\begin{tabular}{||c|c|c|c|c||}
\hline \multicolumn{5}{|c|}{ Maximum patch radius $=1200 \mathrm{~m}$, Slant Range, $r_{0}=10,000 \mathrm{~m}$} \\
\hline \hline Cross-range & \multicolumn{4}{|c|}{ Wavelength, Center Frequency $f_{0}$} \\
\cline { 2 - 5 } Resolution & $0.01 \mathrm{~m}, 30 \mathrm{GHz}$ & $0.03 \mathrm{~m}, 10 \mathrm{GHz}$ & $0.2 \mathrm{~m}, 1.5 \mathrm{GHz}$ & $0.6 \mathrm{~m}, 0.5 \mathrm{GHz}$ \\
\hline $10 \mathrm{~m}$ & Full Patch & Full Patch & Full Patch & Full Patch \\
$3 \mathrm{~m}$ & Full Patch & Full Patch & 948 & 548 \\
$1 \mathrm{~m}$ & Full Patch & 816 & 316 & 183 \\
$0.3 \mathrm{~m}$ & 424 & 245 & 95 & 55 \\
$0.15 \mathrm{~m}$ & 212 & 122 & 47 & 27 \\
\hline
\end{tabular}

Table 3.2: Scene Radius Limits (meters) From Wavefront Curvature Quadratic Phase Errors

the assumption of a large slant-range relative to patch radius $\left(r_{0} \gg x_{0}^{\prime}\right)$, which leads to the approximation of Equation (3.5). However, this assumption is not a necessary condition of the detailed phase models to be introduced next, and consequently, the calculated phase errors will not necessarily be approximations, except where noted.

\subsection{The Space-Variant Post-Filtering Approach to Wavefront Curvature Correction}

The expression of Equation (3.5) is a closed-form, analytic expression for the spacevariant defocus in terms of the known imaging parameters. It is a small-patch approximation that represents a broadside-mode aperture synthesis only. However, as will be shown in the following chapter, an analytical expression can be derived without these restrictions. That is, a general quadratic phase error expression can be derived that represents all patch sizes and slant ranges, for squinted as well as broadside collections. This analytic expression suggests that it is not necessary to live with the patch size restrictions suggested by Equation (3.8). Indeed, Equation (3.5) represents the Fourier transform of the approximated space-variant blur function (ie: the transform of the blurring convolution kernel) at a point $\left(x_{0}^{\prime}, y_{0}^{\prime}\right)$ for the given slant range, frequency extent (resolution), and radar wavelength specified 
by the imaging scenario. The conjugate multiplication of this blur function with the appropriate Fourier extent at $\left(x_{0}^{\prime}, y_{0}^{\prime}\right)$ results in the space-variant deconvolution (refocus) of that portion of the imaged scene upon inverse Fourier transformation.

The full specification of this filter is based on the sophisticated point target phase return model, the analysis of which describes the geometric distortion and defocus in terms of linear and higher order terms. These are the terms which render invalid the tomographic relationship between the polar-formatted phase history data and the imaged scene. The negation of these terms compensates the defocus induced by the assumption of planar wavefronts in the tomographic model. These concepts will be described in chapter 4 . Furthermore, it will be necessary to apply this filter such that the computation is not overly burdensome, while still adequately compensating the defocus effects. The application of this filter, in terms of tradeoffs between the quality of refocus and computational burden, will be covered in Chapter 6. Given enough computation time, any scene can be adequately rid of wavefront curvature defocus effects, regardless of the imaged patch size. Fortunately, as will be shown, adequate refocus can be accomplished in many imaging scenarios with as little as thirty percent additional computation time. This puts the polar-format algorithm in the playing field with many other spotlight-mode imaging algorithms in terms of wavefront curvature compensation, as well as computational load. For certain imaging scenarios, particularly those requiring squinted collections, PFA may be particularly advantageous in terms of computational burden. 
Chapter 3. The Planar Wavefront Assumption in Polar-Formatted Imagery

This page intentionally left blank 


\section{Chapter 4}

\section{Analysis of Phase Errors Arising From Wavefront Curvature}

The tomographic paradigm, first proposed by Munson $[4,5]$, has been used to describe the spotlight-mode phase history data as a two-dimensional (planar) annulus representing the Fourier transform of the three-dimensional scene reflectivity, as projected onto this plane [6]. Consequently, after proper polar-to-rectangular interpolation, the Fourier inversion of this data yields a complex-valued, two-dimensional image domain representation of the illuminated scene. This representation is not an exact portrayal of the imaged scene since several assumptions have been made in the tomographic model which carry over from Walker's original spotlight-mode SAR formulation [2]. These assumptions, when violated, render invalid the direct Fourier relationship between the phase history data and the image domain reconstruction via the tomographic paradigm. One assumption is that the residual quadratic phase error arising from the range-deramp process is insignificant and can be ignored. In fact, this is not the case in the range-dechirp processing of high bandwidth range pulses (large chirp rate $\alpha$ ), particularly when imaging at close range and in conjunction with large patch sizes and high cross-range resolutions $[4,9]$. The effect of this deramp residual phase error is to distort and defocus the imagery in a similar fashion 
as wavefront curvature phase errors. While the models introduced in this chapter can be used to derive the deramp residual phase errors, these are typically small and are usually ignored [2] [7, pp. 363-365]. Instead, the focus of this chapter is to quantify the wavefront curvature phase errors arising from the other shortcoming of the tomographic model; namely, the faulty assumption of planar EM wavefronts illuminating the imaged scene. As opposed to the deramp residual phase errors, the wavefront curvature defocus effects can not be ignored in many common SAR imaging scenarios [12, pp. 435-439].

The previous chapter previewed the nature of the phase errors leading to the distortion and defocus of polar-formatted spotlight mode imagery exhibiting significant wavefront curvature. It was shown that the geometric distortion results from targets lying along the actual arc of the EM wavefront as opposed to residing on the planar wavefront being approximated. In certain imaging scenarios where the differential wavefront curvature is significant, the resultant geometric distortion is severe. However, the planar wavefront model presented was insufficient for the analysis of the defocus due to quadratic phase errors. In certain imaging scenarios, the defocus effects are significant and their space-variant nature makes them troublesome to negate. The analysis of the higher-order phase errors that induce space-variant defocus requires the modeling of the Fourier domain phase history as the collective integration of phase returns from all the targets in the scene. This model will be introduced next and it will reveal the effects of the faulty planar wavefront assumption in terms of residual phase terms that increase in value as the planar wavefront assumption becomes less suitable. From this model, the Fourier domain linear phase terms will be derived; the presence of which serve to place the target in its correct location within the image domain scene. In addition, the residual linear phase terms that induce geometric distortion will be determined, as well as the quadratic term (an approximation of which was given in Equation (3.5)), which leads to the space-variant defocus of the imaged scene. Once these values have been quantified, a method is introduced for the space-variant post-filtering of the imaged scene in 
order to compensate the space-variant defocus, as described in Chapter 5. The effect of this filter is to negate the residual quadratic phase error, thereby refocusing the image.

\subsection{Point Target Contribution to Fourier Domain Reflectivity Function}

The tomographic paradigm states that the demodulated (range) echo of each transmitted pulse evaluates the three-dimensional Fourier transform of the scene reflectivity function, along a line determined between the scene center and platform position. It has been argued, via the projection-slice theorem, that the combined effect of many such projections collected along a straight-line aperture is a two-dimensional (slant) plane representing a slice of the Fourier domain that depicts an orthogonal projection of the three-dimensional scene onto this plane. These ideas will be formalized to arrive at an expression for the phase of this two-dimensional Fourier data collection, as predicted by the tomographic model. Subsequently, this expression will be represented in terms of its Taylor series expansion, and the residual phase error terms will be revealed. Before hand, it is instructive to consider the contribution of a target to the overall scene reflectivity function. That is, to understand the relationship between a target's spatial scene position and its behavior in the Fourier domain. Then, the concept of Fourier domain phase error and its effect on the imaged scene can be fully understood.

This discussion first considers the orthogonal projection of a scene point $\boldsymbol{p}$ into the two-dimensional Fourier slant plane. The contribution of the ideal point target $\boldsymbol{p}$ to the two-dimensional slant plane scene reflectivity can be represented in terms of a delta function as follows:

$$
s_{p}\left(x^{\prime}, y^{\prime}\right)=A_{p} \delta\left(x^{\prime}-x_{0}^{\prime}, y^{\prime}-y_{0}^{\prime}\right)
$$


where $A_{p}$ represents a complex-valued scalar denoting the amplitude and phase of the radar return. At this point, $A_{p}$ is assumed as having some value, without yet knowing the exact derivation of its phase. In the slant plane Fourier domain, this point target contributes a two-dimensional complex sinusoid of the form

$$
\begin{aligned}
S_{p}\left(X^{\prime}, Y^{\prime}\right) & =\mathcal{F}\left\{s_{p}\left(x^{\prime}, y^{\prime}\right)\right\} \\
& =\mathcal{F}\left\{A_{p} \delta\left(x^{\prime}-x_{0}^{\prime}, y^{\prime}-y_{0}^{\prime}\right)\right\} \\
& =A_{p} e^{j\left(x_{0}^{\prime} X^{\prime}+y_{0}^{\prime} Y^{\prime}\right)}
\end{aligned}
$$

where $X^{\prime}$ and $Y^{\prime}$ signify the spatial frequency extent in cross-range and range, respectively. An equivalent vector expression for this Fourier domain point target response is

$$
\bar{S}_{p}(k)=A_{p} e^{j p \cdot k}
$$

where $k$ represents position in the Fourier domain and $p$ is a position vector for the point in the image domain, as projected into the slant plane. These position vectors will now be described in detail. Consider the notion that each demodulated pulse consists of evaluating the Fourier transform of the scene function along a line of the slant plane. A vector denoting a position in this Fourier space and oriented along the line in question can be written in the form

$$
k_{\theta t}^{\prime}=k_{t} u_{\theta}
$$

The vector $\boldsymbol{u}_{\theta}$ is a unit length pointing vector with a directional orientation as shown in Figures 4.1 (a) and (b), which is coincident with the pulse being transduced by the radar platform at the aperture position $\theta$. This vector is then qualified as a point by the specification of the subscript $t$, which denotes time variation within a given return pulse. The magnitude of the unit vector $\boldsymbol{u}_{\theta}$ at a particular intra-pulse time $t$ is

$$
k_{t}=\frac{2 \omega_{0}}{c}+\frac{4 \alpha}{c}\left(t-\tau_{0}\right), \quad\left|t-\tau_{0}\right| \leq T / 2
$$


where $\omega_{o}$ is the radar center frequency, $\alpha$ is the chirp rate, and $\tau_{0}$ is the time delay used to demodulate the return signal of period $T$. One source for the derivation of $k_{t}$ (Equation (4.5)) is [7, pp. 16-26]. During the SAR collection, the vector $k_{\theta t}^{\prime}$ sweeps out a two-dimensional surface within the three-dimensional Fourier domain as its angle $\theta$ and magnitude $k_{t}$ vary. Since the flight path is assumed to be linear, this surface is planar and its orientation analogous to the spatial slant plane defined by the flight line and point at the scene center. The two-dimensional collection geometry and corresponding Fourier domain for this situation are depicted in Figures 4.1 (a) and (b), respectively. It follows from Equation (4.3) that the demodulated return signal sampled at aperture position $\theta$ and time $t$ due to a point target at scene position $p$ is of the form

$$
d_{p}(\theta, t)=A_{p} e^{j p^{\prime} \cdot k_{\theta t}^{\prime}}
$$

Here, the target position vector $\boldsymbol{p}=x_{0}^{\prime} x^{\prime}+y_{0}^{\prime} y^{\prime}$ is the projection of $\boldsymbol{p}$ into the slant plane. By expressing $\boldsymbol{p}^{\prime}$ and $\boldsymbol{k}^{\prime}$ in terms of their respective Cartesian $x-y$ coordinates via Equation (4.2), the point target response can be written as

$$
d_{x_{0}^{\prime}, y_{0}^{\prime}}\left(X^{\prime}, Y^{\prime}\right)=A_{p} e^{j\left(x_{0}^{\prime} X^{\prime}+y_{0}^{\prime} Y^{\prime}\right)} .
$$

This result shows that the phase function is linear in each of the slant plane spatial frequency coordinates $X^{\prime}$ and $Y^{\prime}$, representing azimuth and range, respectively. Furthermore, the phase function is proportional to the target location $\left(x_{0}^{\prime}, y_{0}^{\prime}\right)$ in the slant plane coordinates of the scene. According to the tomographic paradigm, the SAR data from a point target consists of a complex sinusoid whose frequency in two dimensions corresponds to the location of the target projected into the slant plane. This statement is consistent with the known Fourier transform of an ideal impulse response function, which corresponds to the sinusoid of a frequency proportional to the spatial displacement of the impulse from the origin. Thus, it should now be clear that the linear phase terms of the Fourier domain phase history serve to identify the position of the particular target inducing those phase terms. By the simple inverse 


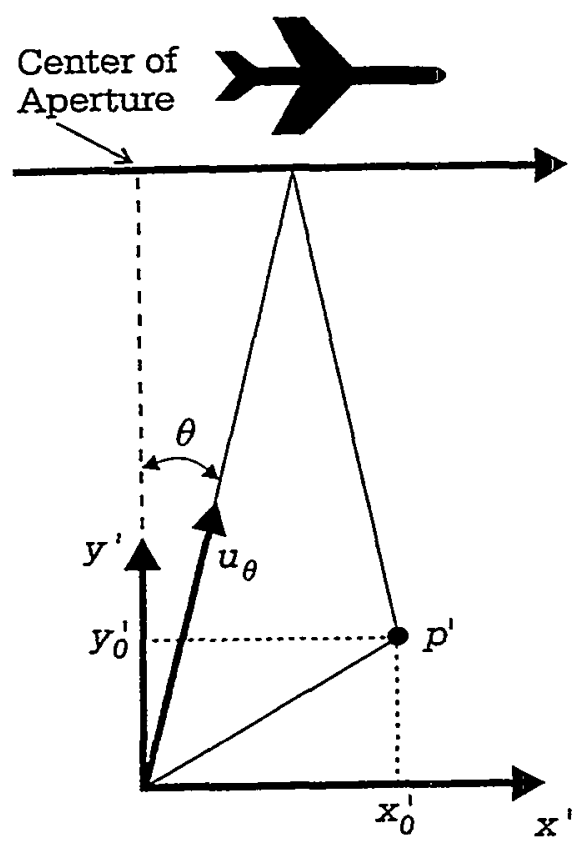

(a)

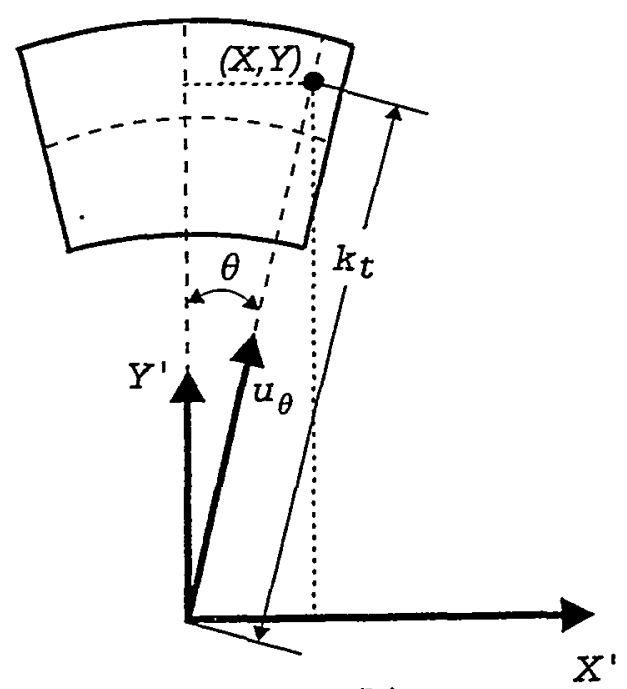

(b)

Figure 4.1: Slant Plane Imaging Geometry for Point Target Return

Fourier transform of the phase history data, an image is produced of this target and, by extension via superposition, the entire scene is formed. This is assuming, of course, the interpolation of the annular phase history data onto the Cartesian grid specified by $X^{\prime}$ and $Y^{\prime}$, prior to Fourier inversion. The previous discussion and mathematical development has been adapted from [7, pp. 355-358]

As the prior discussion points out (and is summarized in Equation (4.7)), the linear Fourier phase terms of the form

$$
\phi_{1 x_{0}^{\prime}, y_{0}^{\prime}}\left(X^{\prime}, Y^{\prime}\right)=x_{0}^{\prime} X^{\prime}+y_{0}^{\prime} Y^{\prime}
$$

serve to place the target in its correct position within the imaged slant plane scene. More simply, the relation may be expressed as

$$
\phi_{1}=x_{0}^{\prime} X^{\prime}+y_{0}^{\prime} Y^{\prime}
$$

and from Equation (4.7), the point target position is

$$
d_{x_{0}^{\prime}, y_{0}^{\prime}}\left(X^{\prime}, Y^{\prime}\right)=A_{p} e^{j \phi_{1}}
$$


However, as will be shown next, the assumption of planar wavefronts in the tomographic paradigm leads to extraneous linear terms. These are the terms which render invalid the direct Fourier relationship between the phase history and the scene being illuminated. Depending on the particular imaging scenario, any increase in the residual differential wavefront curvature leads to larger extraneous linear phase terms and the geometric distortion present in the processed image increases accordingly. The linear distortion phase terms arising from the planar wavefront approximation, if they are present, are noted as

$$
\phi_{1 \epsilon}=f_{\hat{x}}\left(x_{0}^{\prime}, y_{0}^{\prime}\right) X^{\prime}+f_{\hat{y}}\left(x_{0}^{\prime}, y_{0}^{\prime}\right) Y^{\prime}
$$

and the total linear phase is

$$
\begin{aligned}
\phi_{1 t} & =\phi_{1}+\phi_{1 \epsilon} \\
& =x_{0}^{\prime} X^{\prime}+y_{0}^{\prime} Y^{\prime}+\phi_{1 \epsilon} \\
& =x_{0}^{\prime} X^{\prime}+y_{0}^{\prime} Y^{\prime}+f_{\hat{x}}\left(x_{0}^{\prime}, y_{0}^{\prime}\right) X^{\prime}+f_{\hat{y}}\left(x_{0}^{\prime}, y_{0}^{\prime}\right) Y^{\prime} .
\end{aligned}
$$

It is assumed that all linear phase terms, including those for correct placement of the target as well as the extraneous error terms leading to distortion, are a function of the slant plane point target location $\left(x_{0}^{\prime}, y_{0}^{\prime}\right)$. Thus, Equation (4.12) can be expressed as

$$
\phi_{1 t}=x_{0}^{\prime} X^{\prime}+y_{0}^{\prime} Y^{\prime}+f_{\tilde{x}^{\prime}} X^{\prime}+f_{\tilde{y}^{\prime}} Y^{\prime}
$$

The assumption of planar wavefronts also leads to quadratic (and higher order) phase terms, which serve to defocus the imagery processed via the polar-format algorithm. This defocus is space variant and can not be compensated via a postwarping procedure. The higher order phase terms serve to broaden the IPR of the point target, and consequently degrade the image resolution. As is shown in Appendix A, the degree of IPR broadening can be expressed in terms of the peak quadratic phase error at the point of the IPR. Since this phase error will be shown to be a space-varying one with respect to point target location, the overall effect of 
the quadratic phase error is to spatially vary the defocus of the image. As opposed to the linear phase case, there are no quadratic (or higher order) phase terms that serve to specify the point target position. In fact, any higher order phase terms that are present serve only to defocus the image. If these terms are significant, they must be adequately compensated in order to avoid noticeable defocus of the processed imagery. Since any second-order phase term is an error term,

$$
\phi_{2 t}=\phi_{2 \epsilon}=\phi_{2}
$$

and the quadratic phase error can be represented in terms of its individual contributions

$$
\begin{aligned}
\phi_{2 t} & =f_{\widehat{x x}^{\prime}} X^{\prime} X^{\prime}+f_{\overparen{x y^{\prime}}} X^{\prime} Y^{\prime}+f_{\widehat{y y^{\prime}}} Y^{\prime} Y^{\prime} \\
& =f_{\widehat{x}^{\prime}} X^{\prime 2}+f_{\overparen{x y^{\prime}}} X^{\prime} Y^{\prime}+f_{\widehat{y}^{\prime}} Y^{\prime 2},
\end{aligned}
$$

where $f_{\widehat{x^{\prime}}}, f_{\widehat{x y^{\prime}}}$ and $f_{\overparen{y^{2}}}$ are unique functions of the target point $\left(x_{0}^{\prime}, y_{0}^{\prime}\right)$ for the phase history frequency extent $\left(X^{\prime}, Y^{\prime}\right)$. The total phase contribution of all first and second-order terms is

$$
\begin{aligned}
\phi_{t} & =\phi_{1 t}+\phi_{2 t} \\
& =\phi_{1 t}+\phi_{2 \epsilon} \\
& =\phi_{1}+\phi_{1 \epsilon}+\phi_{2 \epsilon} \\
& =x_{0}^{\prime} X^{\prime}+y_{0}^{\prime} Y^{\prime}+f_{\hat{x}^{\prime}} X^{\prime}+f_{\hat{y}^{\prime}} Y^{\prime}+f_{\widehat{x^{2}}} X^{\prime 2}+f_{\widehat{x y^{\prime}}} X^{\prime} Y^{\prime}+f_{\widehat{y^{\prime}}} Y^{\prime 2}
\end{aligned}
$$

while keeping in mind that $\phi_{1}=x_{0}^{\prime} X^{\prime}+y_{0}^{\prime} Y^{\prime}$ correctly places the point in the imaged scene and that all other terms are error terms. Thus, the total phase error (through second-order) is $\phi_{t}-\phi_{1}$ and is found to be

$$
\begin{aligned}
\phi_{t \epsilon} & =\phi_{1 \epsilon}+\phi_{2 t} \\
& =\phi_{1 \epsilon}+\phi_{2 \epsilon} \\
& =f_{\hat{x}^{\prime}} X^{\prime}+f_{\hat{y}^{\prime}} Y^{\prime}+f_{\widehat{x^{2}}} X^{\prime 2}+f_{\overparen{x y^{\prime}}} X^{\prime} Y^{\prime}+f_{\widehat{y^{\prime}}} Y^{\prime 2}
\end{aligned}
$$




\subsection{The Phase Return From a Point Target}

The relationship between phase and point target location is now known. Equation (4.7) conveys in a simple and concise way, the very essence of the SAR imaging process. Further analysis has shown that the first-order (linear) phase history terms $x_{0}^{\prime} X^{\prime}$ and $y_{0}^{\prime} Y^{\prime}$ of Equation (4.16) serve to correctly place the target in the imaged scene in cross-range and range, respectively. However, according to the tomographic development, the approximation of spherical wavefronts contributes to linear, quadratic, and higher order phase terms that serve to distort and defocus the imagery. The residual linear terms $f_{\hat{x}^{\prime}} X^{\prime}$ and $f_{\hat{y}^{\prime}} Y^{\prime}$ arise from the planar wavefront assumption and serve to distort the processed imagery in cross-range and range, respectively. The residual linear range term serves to induce the slant plane range sag $\Delta y^{\prime}$ as described in the previous chapter, such that $\Delta y^{\prime}=f_{\hat{y}^{\prime}}$. The quadratic phase terms (if present) serve to defocus the image and it will soon be shown that this defocus is space-variant and in the cross-range only, because all quadratic phase terms are zero except $f_{\widehat{x^{2}}} X^{\prime 2}$, the cross-range quadratic term.

The examples of Chapter 3 served to illustrate the effects of these phase error terms in a qualitative and behavioral manner. However, what is the actual phase generated by a target for a given imaging scenario? The impact of the phase terms has been observed and analyzed, though no attempt has yet been made to quantify the phase errors, as based on actual imaging scenarios. Without knowledge of the amount of phase error present, these errors can not be compensated, and consequently the imagery can not be geometrically rewarped or refocused.

\subsubsection{Broadside Phase Error Modeling}

The derivation of a closed-form solution for the linear and quadratic phase requires a revisit to the point target contribution model of the previous section. This time, an emphasis is put on the precise radar echo in the context of the actual imaging 
geometry involved. This section describes the work of Jakowatz, et al [7, pp. 355360], who takes the following approach to phase error model. Consider the slant plane imaging geometry of Figure 4.2. The target position designated by $p^{\prime}$ is again the projection of the actual target location from its native three-space into the slant plane. It is important to realize that this projection is not an orthogonal projection, as implied earlier. Instead, it is a projection along a circular arc in three dimensions centered on and normal to the flight path. That is, centered along a contour of constant range and range rate. The consequence of assuming an orthogonal projection is the a slight geometric distortion of this image with respect to the proper, circularly arced projection. This distortion is easily corrected after image formation, assuming a geometrically proper slant plane image is required, relative to the ground plane. This distortion arises from the assumption of an orthogonal projection of the threedimensional imagery into the slant plane. It is independent of the two-dimensional, slant plane geometric distortion due to wavefront curvature that is addressed here. Consequently, it will be ignored since this discussion deals with wavefront curvature compensation in the slant plane, and not those errors associated with the $3 \mathrm{D}$ to $2 \mathrm{D}$ projection of the ground plane imagery to the slant plane.

Assume the radar transmits a linear FM chirp pulse of duration $T$ and represented by

$$
s_{x}(t)=\Re\left\{e^{j \phi_{x}(t)}\right\}, \quad|t| \leq T / 2
$$

whose phase function is a quadratic of the form

$$
\phi_{x}(t)=\omega_{0} t+\alpha t^{2}
$$

where $\omega_{0}$ is the radar center frequency and $\alpha$ is the chirp rate. Consider a point target $p^{\prime}$ in the scene at distance $r_{t}$ from the radar at a certain point in the aperture, as denoted by angle $\theta$ and distance $z^{\prime}$ in Figure 4.2 (a). The phase of the radar return or echo from this target at aperture position $\theta$ is simply an appropriately delayed replica of the transmitted phase of Equation (4.19), and is expressed by

$$
\phi_{\tau_{t}}(t, \theta)=\omega_{0}\left(t-2 r_{t} / c\right)+\alpha\left(t-2 r_{t} / c\right)^{2} .
$$




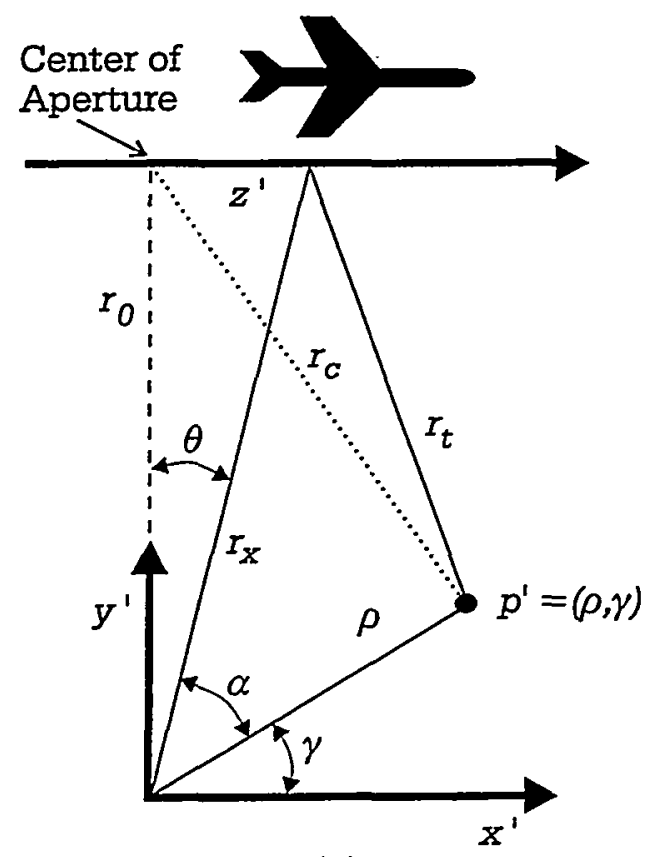

(a)

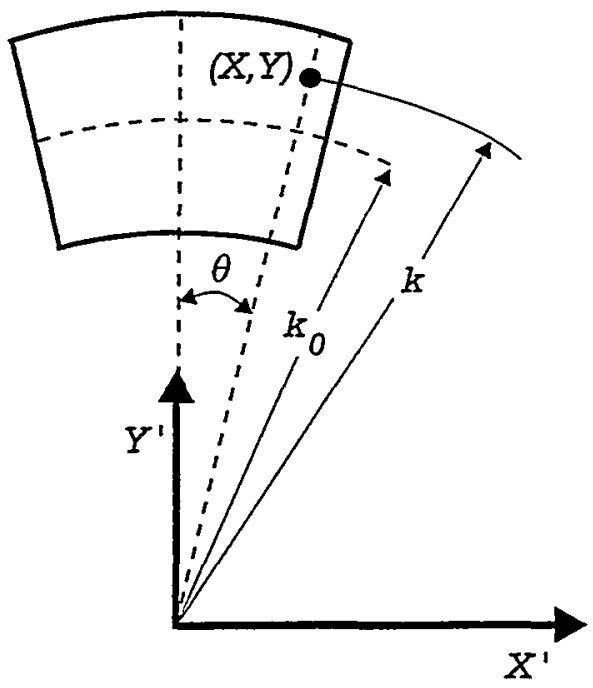

(b)

Figure 4.2: Broadside Geometric Model for Derivation of Wavefront Curvature Phase Errors

The time delay is represented as a function of distance from the platform to the target point, $r_{t}$. This distance is inferred to be a function of the platform position $\cdot \theta$. By definition, as a phase coherent SAR imaging system, the local reference used to demodulate the return signal has exactly the same phase function as the return from a hypothetical target at the scene center (CRP). When the platform is at an aperture position $\theta$ and corresponding distance $r_{x}$ from the CRP, as shown in Figure 4.2 (a), the reference phase is a variation of Equation(4.20) and is given by

$$
\phi_{r_{x}}(t, \theta)=\omega_{0}\left(t-2 r_{x} / c\right)+\alpha\left(t-2 r_{x} / c\right)^{2} .
$$

The process of quadrature demodulation forms a complex video signal represented in separate $I$ and $Q$ components. The phase of this signal is the difference of the 
return and the reference signal phases:

$$
\begin{aligned}
\dot{\phi}(t, \theta) & =\phi_{r_{t}}(t, \theta)-\phi_{r_{x}}(t, \theta) \\
& =-\frac{2}{c}\left(\omega_{0}+2 \alpha t\right)\left(r_{t}-r_{x}\right)+\frac{4 \alpha}{c^{2}}\left(r_{t}^{2}-r_{x}^{2}\right) \\
& =-\frac{2}{c}\left[\omega_{0}+2 \alpha\left(t-\frac{2 r_{x}}{c}\right)\right]\left(r_{t}-r_{x}\right)+\frac{4 \alpha}{c^{2}}\left(r_{t}-r_{x}\right)^{2} .
\end{aligned}
$$

This equation is an exact expression for the phase of the complex video signal obtained at aperture angle $\theta$ in terms of the radar parameters and the relative geometry of the target and platform. The equation can be rewritten in the form

$$
\bar{\phi}(\theta, k)=-k\left(r_{t}-r_{x}\right)+\frac{4 \alpha}{c^{2}}\left(r_{t}-r_{x}\right)^{2},
$$

where

$$
k=\left[\frac{2 \omega_{0}}{c}+\frac{4 \alpha}{c}\left(t-\frac{2 r_{x}}{c}\right)\right]
$$

denotes a scaled and offset measure of intra-pulse time $t$. This equation for $k$ is identical to that given by Equation (4.5) except in this case, the time index $k$ is a function of imaging geometry; specifically, the distance of the platform from scene center. This mathematical development has been adapted from [7, pp. 358-360]

Consider a certain sample of the video signal, with phase $\bar{\phi}(\theta, k)$, at a time index $k$ in a return pulse at aperture angle $\theta$. This sample $(X, Y)$ is laid down in a twodimensional array at angular coordinate $\theta$ and radial position $k$, as shown in Figure 4.2 (b). As $\theta$ spans the extent of the aperture and as $k$ varies throughout the duration of each return pulse, a polar annulus is swept out in the two dimensional phase space. By the tomographic paradigm, as discussed in Chapter 2, this space is known to represent the band-limited Fourier transform of the scene reflectivity function for the illuminated patch. By the very same paradigm, this phase space is also understood to contain certain phase components that render invalid the direct relationship between this phase history and Fourier transformation of the reflectivity function. However, for many imaging scenarios, this phase history estimate is precise enough to allow the 
use of the mathematically convenient and numerically efficient Fourier techniques to form an acceptable image, if within the patch size limits specified by Equation (3.8) in Chapter 3.

For those scenarios in which the patch size limits are violated, significant differential wavefront curvature is present. Consequently, the assumption of strictly planar wavefronts illuminating the scene is rendered invalid; thus linear, quadratic, and higher order residual phase terms appear. The nature of these phase errors and their mathematical representation is now well understood, as are their visual effects on the polar-format processed imagery. What remains to be determined are the specific values of these phase errors in the Fourier domain as a function of the point target position in the imaged scene, given a particular imaging scenario. With this knowledge, a filter can be designed and implemented to compensate the residual quadratic phase error and its associated space-variant defocus.

The second addend of Equation (4.24); specifically, the term $\left(4 \alpha / c^{2}\right)\left(r_{t}-r_{x}\right)^{2}$, represents a phase error resulting from a residual of the deramp process. This term leads to the deramp residual phase error of the scene, which in fact is based on linear and higher order phase terms which serve to distort and defocus the image, in a similar manner as wavefront curvature. As with wavefront curvature, the deramp residual phase error is ignored in the tomographic model. However, since deramp phase errors are scaled by a factor of $1 / c^{2}$, they are typically small in magnitude and can be ignored, except for particularly high chirp bandwidths or very short slant range collections. The derivation and subsequent compensation of deramp residual phase errors are not the subject of this dissertation, although the process is quite similar to that which will be discussed for wavefront curvature compensation. The reader is directed to the following references for details on deramp residual phase errors and their compensation, which is known as deskew processing or deskewing: $[2,4,7$, pp. 363-365] and $[8,9]$. The analysis of phase errors associated with wavefront curvature is independent of the deramp analysis. Consequently, ignoring the deramp residual phase errors, the expression of Equation (4.24) representing the phase of the 
complex video signal is now

$$
\begin{aligned}
\dot{\phi}(\theta, k) & =-k\left(r_{t}-r_{x}\right) \\
& =k\left(r_{x}-r_{t}\right),
\end{aligned}
$$

where $k$ is unchanged from before and shown to be

$$
k=\left[\frac{2 \omega_{0}}{c}+\frac{4 \alpha}{c}\left(t-\frac{2 r_{x}}{c}\right)\right] .
$$

\subsubsection{Phase of Video Signal with Respect to Imaging Geom- etry}

At this point, nothing more has been done than to arrange the two-dimensional phase space of Figure 4.2 (b) according to the aperture position $\theta$ of the radar platform at the time index $k$, which is a function of the distance $r_{x}$ from the scene center to the platform and the distance $r_{t}$ from the platform to the target. These parameters define the phase space $\phi(\theta, k)$ as given by Equation (4.26), which appropriately neglects deramp residual phase errors. However, in order to design the space-variant postfilter for compensating the defocus due to wavefront curvature, is desirable to express the Fourier phase history domain $\left(X^{\prime}, Y^{\prime}\right)$ in terms of a hypothetical point target $\left(x_{0}^{\prime}, y_{0}^{\prime}\right)$. That is, to represent the phase in the form of $\phi_{x_{0}^{\prime} y_{0}^{\prime}}\left(X^{\prime}, Y^{\prime}\right)$, as was presented in Section 4.1. This requires the further coupling (relation) of Figures 4.2 (a) and (b), as follows. From Figure 4.2 (b), let the phase history radius $k$ be represented by its Cartesian frequency coordinates $(X, Y)$ such that

$$
k=\sqrt{X^{2}+Y^{2}}
$$

and the aperture position $\theta$ relative to the scene center is given by

$$
\theta=\tan ^{-1}\left(\frac{X}{Y}\right)
$$

Consequently,

$$
\tan (\theta)=\frac{X}{Y}, \quad Y>0
$$


As usual, this analysis is performed in the slant plane. The prime is dropped at this point in the mathematical development since the point $(X, Y)$ actually represents a Cartesian point in the Fourier space of $\left(X^{\prime}, Y^{\prime}\right)$. The analogous spatial representation of the aperture position $\theta$ is shown in Figure 4.2 (a) and is given by

$$
\theta=\tan ^{-1}\left(\frac{z^{\prime}}{r_{0}}\right)
$$

Consequently,

$$
z^{\prime}=r_{0} \tan (\theta), \quad r_{0}>0
$$

and from Equation (4.30),

$$
z^{\prime}=\frac{r_{0} X}{Y}, \quad r_{0}>0, Y>0
$$

and

$$
z^{2}=r_{o}^{2}\left(\frac{X}{Y}\right)^{2}
$$

The complex video signal phase of Equation (4.26) is represented in terms of $k, r_{x}$ and $r_{t}$. The $k$ term represents the scaled and offset measure of intra-pulse time $t$ and is represented by Equation (4.27) in terms of the radar center frequency $\omega_{0}$, range LFM chirp rate $\alpha$, and the distance from platform to radar scene center, $r_{x}$. This distance is measured from the scene center to the radar platform at a arbitrary aperture point. In order to represent the complex video phase in terms of this arbitrary point in the scene, the distances $r_{x}$ and $r_{t}$ must be defined relative to the aperture position $\theta$ and the point target location $p^{\prime}$, where $p^{\prime}=(\rho, \gamma)$. The distance $r_{x}$ is defined as follows: A right triangle exists between the edges $r_{o}, r_{x}$ and $z^{\prime}$ in Figure 4.2 (a). The distance $r_{0}$ is the broadside range from platform to scene center. By the Pythagorean relation,

$$
r_{x}^{2}=z^{2}+r_{o}^{2}
$$


and by substitution from Equation (4.34),

$$
\begin{aligned}
r_{x}^{2} & =r_{0}^{2}\left(\frac{X}{Y}\right)^{2}+r_{0}^{2} \\
& =r_{0}^{2}\left\{1+\left(\frac{X}{Y}\right)^{2}\right\}
\end{aligned}
$$

and

$$
r_{x}=r_{0} \sqrt{1+\left(\frac{X}{Y}\right)^{2}}
$$

Furthermore, from Equation (4.30),

$$
r_{x}=r_{0} \sqrt{1+\tan ^{2}(\theta)}
$$

The distance $r_{t}$ is defined from an arbitrary platform position along the aperture to the target position $p^{\prime}$ in the slant plane. From Figure 4.2 (a), this distance can be derived using the law of cosines on the triangle defined by the edges $r_{x}, \rho$ and $r_{t}$ :

$$
r_{t}^{2}=r_{x}^{2}+\rho^{2}-2 \rho r_{x} \cos (\alpha)
$$

However, $\cos (\alpha) \triangleq \sin \left(\frac{\pi}{4}-\alpha\right)=\sin (\theta+\gamma)$, so

$$
r_{t}^{2}=r_{x}^{2}+\rho^{2}-2 \rho r_{x} \sin (\theta+\gamma)
$$

and taking the square root of both sides,

$$
r_{t}=\sqrt{r_{x}^{2}+\rho^{2}-2 \rho r_{x} \sin (\theta+\gamma)}
$$

From Equation (4.29), $r_{t}$ can also be represented by

$$
r_{t}=\sqrt{r_{x}^{2}+\rho^{2}-2 \rho r_{x} \sin \left(\arctan \left(\frac{X}{Y}\right)+\gamma\right)}
$$

At this point, the phase of the complex video signal $\phi(\theta, k)$ is defined as a function of the imaging aperture position $\theta$, the time index $k$, and a target in the imaged scene at a polar point $p^{\prime}=(\rho, \gamma)$. In summary:

$$
\phi_{p^{\prime}}(\theta, k)=k\left(r_{x}-r_{t}\right)
$$


where

$$
\begin{aligned}
k & =\left[\frac{2 \omega_{0}}{c}+\frac{4 \alpha}{c}\left(t-\frac{2 r_{x}}{c}\right)\right], \\
r_{x} & =r_{0} \sqrt{1+\tan ^{2}(\theta)}
\end{aligned}
$$

and

$$
r_{t}=\sqrt{r_{x}^{2}+\rho^{2}-2 \rho r_{x} \sin (\theta+\gamma)}
$$

The phase history swept out as $\theta$ and $k$ are varied represents the Fourier space for the target $p^{\prime}$ located at $(\rho, \gamma)$, as shown in Figure 4.2 (a). The frequency extent in range and azimuth, $\Delta X^{\prime}$ and $\Delta Y^{\prime}$, are determined by the aperture extent $\Delta \theta$ and chirp bandwidth, respectively, as described in Chapter 2. Furthermore, these frequency extents dictate the scene resolution as described by Equations (2.18) and (2.19). A complete Fourier space representing all targets present in an imaged scene would consist of the superposition of the phase histories for each individual point target.

\subsubsection{Synthetic Target Generation}

The synthetic target generator (SYNTARG), described in [7, pp. 391-414], emulates the motion of the SAR and mathematically transmits, receives, and demodulates the returns of a number of point reflectors. It creates a two-dimensional phase history from this collection of point targets, based on Equation (4.43), which closely matches the signals that an actual SAR would obtain under similar circumstances. The synthesized dataset can then be processed in various ways as to quantify the image properties obtained by various image formation methods. Specifically, as will be shown next, the synthetic target generator phase history includes the terms responsible for the distortion and space-variant defocus of the imaged scene resulting from the planar wavefront assumption. Consequently, SYNTARG can be used to simulate the close-in, low frequency, high resolution, or wide patch scenarios that exhibit these 
significant wavefront curvature anomalies. Furthermore, the space-variant post-filter for wavefront curvature correction can be applied to these synthetic data sets, and the effectiveness of this filter can be analyzed on a number of different scenarios without actually using a real aircraft and SAR to generate these data sets. An added benefit of processing SYNTARG data is the avoidance of the atmospheric effects and platform motion errors that occur in actual SAR imaging scenarios. Also, the IPRs generated by SYNTARG are theoretically ideal; therefore, the ability of the spacevariant post-filter to compensate wavefront curvature effects can be analyzed more quantitatively. Most of the remaining examples in this dissertation use SYNTARG data and help to illustrate the benefits of synthetic target generation for testing SAR algorithms.

One disadvantage of SYNTARG is its high computational burden. For example, a phase history of $n \times n$ points is generated from the superposition of $p$ point target phase histories, where $p$ is the number of targets and $n^{2}$ complex phase history points are generated for each target. Clearly, as the number of point targets being simulated increases, the computational burden increases significantly. Thus, the growth of the computational burden is bounded from above by $O\left(p n^{2}\right)$. This asymptotic measure of growth, denoted by $O()$ (big-oh) notation, is described in detail in [84, pp. 23-41]. In Chapter 6, this notation will also be used to describe the computational burden of the space-variant post-filter. At first, the computational burden associated with SYNTARG seems prohibitive. However, SYNTARG obviates the need to acquire real SAR data and furthermore, the synthetic phase histories are ideal in many respects. These advantages generally outweigh the disadvantage of SYNTARG's computing time, as well as eliminating the expense of flying a real SAR. Furthermore, while beyond the scope of this dissertation, it can be shown that the SYNTARG algorithm is quite amenable to parallel processing, thereby reducing computation time. The space-variant post-filter for wavefront curvature correction is based on Equation (4.43), as is SYNTARG, yet SVPF is made more computationally efficient through a number of simplifications, as will be described in the remainder of this chapter and 
in Chapter 5.

Prior to analyzing Equation (4.43) in terms of its first-order, quadratic, and higher-order phase terms, it is useful to form an analogous representation in terms of the frequency extent $\left(X^{\prime}, Y^{\prime}\right)$. This precisely matches the domain of the Fourier phase history; namely, its spatial bandwidth, and allows for the use of the computational simplifications when compensating the wavefront curvature effects. Furthermore, provided the actual flight path of the SAR does not violate the assumption of a linear flight path and that the image is motion compensated to the scene center, it is not necessary to know the geometric position of the sample points along the aperture. That is, the linear flight path assumption leads to a closed-form solution that does not rely on knowledge of individual aperture positions. The slant plane, Fourier domain phase history $\left(X^{\prime}, Y^{\prime}\right)$ in terms of a hypothetical Cartesian point target $\left(x_{0}^{\prime}, y_{0}^{\prime}\right)$ is shown to be

$$
\phi_{x_{0}^{\prime}, y_{0}^{\prime}}\left(X^{\prime}, Y^{\prime}\right)=k\left(r_{x}-r_{t}\right)
$$

where

$$
\begin{aligned}
k & =\left[\frac{2 \omega_{0}}{c}+\frac{4 \alpha}{c}\left(t-\frac{2 r_{x}}{c}\right)\right], \\
r_{x} & =r_{0} \sqrt{1+\left(\frac{X}{Y}\right)^{2}},
\end{aligned}
$$

and

$$
r_{t}=\sqrt{r_{x}^{2}+\rho^{2}-2 \rho r_{x} \sin \left(\arctan \left(\frac{X}{Y}\right)+\gamma\right)}
$$

Furthermore, the Cartesian slant plane representation $\left(x_{0}, y_{0}\right)$ of the point target $p=(\rho, \gamma)$ is given by

$$
x_{0}^{\prime}=\rho \cos \gamma
$$

and

$$
y_{0}^{\prime}=\rho \sin \gamma
$$




\subsection{Polynomial Phase Term Representation}

Section 4.1 describes how the linear phase terms of Fourier domain phase history serve to identify the position of the of the particular point target inducing those terms. By nature of the Fourier transform relationship, these Fourier phase terms represent a complex sinusoid whose frequency in the two-dimensional space $\left(X^{\prime}, Y^{\prime}\right)$ are proportional to the target position $\left(x_{0}^{\prime}, y_{0}^{\prime}\right)$ in the transformed image space. Furthermore, additional residual phase terms in both $X^{\prime}$ and $Y^{\prime}$ appear in the presence of differential wavefront curvature, and serve to geometrically distort the image. These are accompanied by higher order phase terms that induce a space-variant defocus of the image. In [7, pp. 360-363], Equation (4.41) is simplified via the small patch approximation, which assumes the dimensions of the scene being imaged are small compared to the SAR standoff distance, such that $\rho \ll r_{0}$. Consequently, the simplified equation for $r_{t}$, the distance from platform to target, is substituted directly into Equation (4.43) to yield the first and second-order phase terms, for a broadside collection under the small patch constraints. However, it is desirable to derive these phase terms without patch size constraints. To this end, in order to isolate these phase terms, a two-dimensional Taylor series expansion will now be applied to the Fourier domain phase history described by Equation (4.44).

The Taylor series decomposition serves to represent the phase return from a target in terms of an infinite series of linear, quadratic, and higher order components. This method was first used by Walker in 1980 [2] to describe the patch size limitations of wavefront curvature in broadside collections, and it does not require small patch approximations. The unique contribution of this dissertation is the application of the Taylor series decomposition to both squinted and broadside collection geometries, without regard to patch size or standoff range. The broadside case is discussed here, with the generalized, squint-mode scenario presented in Section 4.4. From Equation (4.44), the Taylor series expansion of the phase function $\phi_{x_{0}^{\prime}, y_{0}^{\prime}}\left(X^{\prime}, Y^{\prime}\right)$ expanded 
around the point $\left(X_{0}, Y_{0}\right)$ is

$$
\begin{aligned}
\phi_{x_{0}^{\prime}, Y_{0}^{\prime}}\left(X^{\prime}, Y^{\prime}\right)= & a_{00}+a_{10} X^{\prime}+a_{01} Y^{\prime}+a_{20} X^{\prime 2} \\
& +a_{11} X^{\prime} Y^{\prime}+a_{02} Y^{\prime 2}+\ldots
\end{aligned}
$$

where

$$
a_{i j}=\left.\left(\frac{1}{i ! j !}\right)\left(\frac{\partial^{i+j} k\left(r_{x}-r_{t}\right)}{\partial X^{i} \partial Y^{j}}\right)\right|_{\substack{X=X_{0}, Y=Y_{0}}} .
$$

\subsubsection{Linear Phase Terms}

The constant phase term $a_{00}$ is of no consequence. The terms which are linear in $X^{\prime}$ and $Y^{\prime}$ are image translation terms and specify the linear displacement in azimuth and range in proportion to $a_{10}$ and $a_{01}$, respectively. In the presence of wavefront curvature, these terms contain extraneous linear components that induce the geometric distortion. The second-order terms, namely $a_{20}, a_{11}$ and $a_{02}$, represent the space-variant defocus associated with wavefront curvature. These terms, which arise from the faulty planar wavefront assumption in tomography, will be compensated via the space-variant post-filter. The second-order coefficients rely both on point target location $\left(x_{0}^{\prime}, y_{0}^{\prime}\right)$ and processing aperture position $(\theta, k)$, and historically, this has been considered a computationally difficult post-filtering problem [2, 32]. Alternatively, some have suggested the generation of small patches [85, pp. 274] (and the associated mosaicking, which may prove to be inefficient [12, pp. 483]) in order to avoid wavefront curvature defocus effects. However, the relation of Equation (4.44) associates the motion of the target relative to the platform in terms of the linear aperture shown in Figures 4.2 (a) and (b). Assuming a linear trajectory and that out of plane motion is adequately phase compensated, the coherent phase return from a point target can be represented strictly in terms of its location in the scene and no longer has dependence on the specific aperture positions in the scene, provided the aperture encompasses an angular interval which is sufficiently small. That is, for an angular interval that is much less than $360^{\circ}$, as is typical in SAR but not 


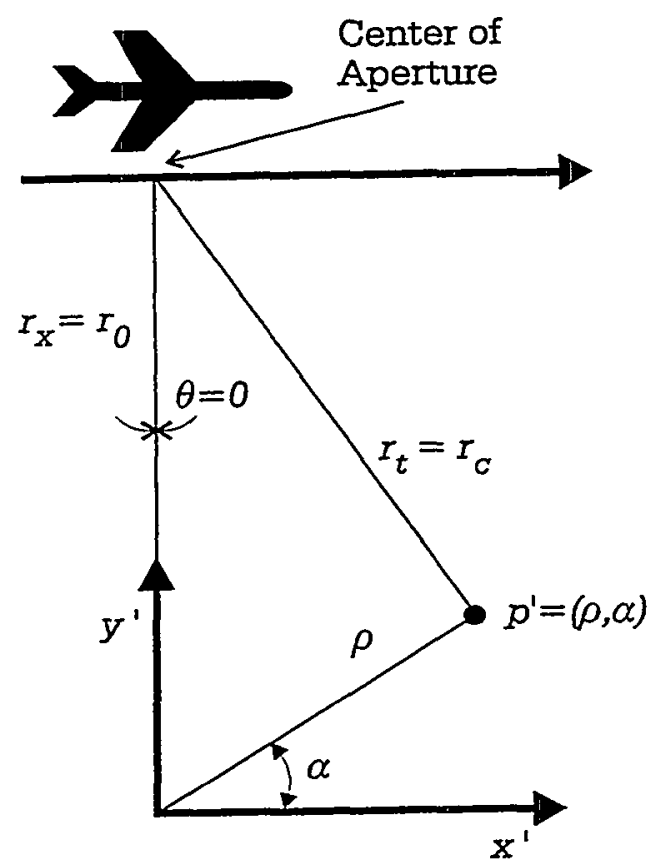

(a)

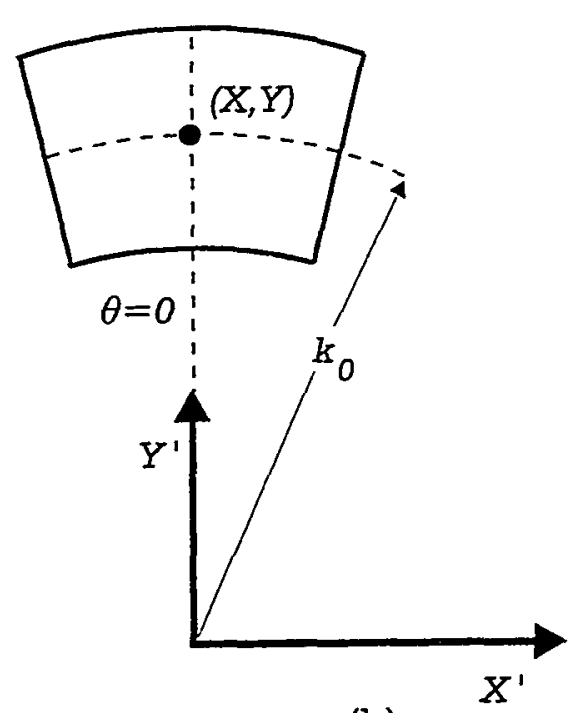

(b)

Figure 4.3: Aperture Center Geometric Model for Derivation of Wavefront Curvature Phase Errors

CAT, only a portion of the phase error $\phi_{x_{0}^{\prime}, y_{0}^{\prime}}$ will contribute to the impulse response broadening and consequent loss of resolution [2].

If the center of the processing aperture is located at $(\theta, k)=\left(0, k_{0}\right)$, where $k_{0}$ is the nominal (spatial frequency) phase history radius, then from Equation (4.38),

$$
\begin{aligned}
r_{x} & =r_{0} \sqrt{1+\tan ^{2}(0)} \\
& =r_{0}
\end{aligned}
$$

and

$$
\begin{aligned}
r_{t} & =\sqrt{r_{0}^{2}+\rho^{2}-2 \rho r_{0} \sin (\gamma)} \\
& =r_{c} .
\end{aligned}
$$

This represents the geometry at aperture center for a broadside (non-squinted) collection and is shown in Figure 4.3. The distance $r_{c}$ represents that from the radar 
platform at mid-aperture to the point target. Since the processing aperture is assumed to encompass a sufficiently small angle, the two-dimensional Taylor series of Equations (4.47) and (4.48) can be expanded around the aperture center such that

$$
\begin{aligned}
(X, Y) & =\left(X_{0}, Y_{0}\right) \\
& =\left(0, k_{0}\right),
\end{aligned}
$$

where

$$
k_{0}=\frac{4 \pi}{\lambda} .
$$

Now, calculating the Taylor terms $a_{10}$ and $a_{01}$ will yield the linear phase terms, while $a_{20}, a_{11}$ and $a_{02}$ will yield the quadratic. Cubic and higher terms are smaller and for sufficiently small processing apertures can be safely ignored. It will be shown via UWB and L-Band SAR examples in Chapter 6 that in fact the higher order terms contribute little to the defocus and can be ignored. The image distortion effects caused by $\phi_{x_{0}^{\prime}, y_{0}^{\prime}}\left(X^{\prime}, Y^{\prime}\right)$ will be examined now. The coefficient $a_{10}$ represents the target position in azimuth. Therefore, this coefficient specifies the proper crossrange position for the target as well as describing the geometric distortion. This coefficient is calculated as follows. From Equation (4.48),

$$
\begin{aligned}
a_{10}= & \frac{\partial \phi_{x_{0}^{\prime}, y_{0}^{\prime}}\left(X^{\prime}, Y^{\prime}\right)}{\partial X} \\
= & -\frac{1}{b_{10} Y\left(X^{2}+Y^{2}\right)} \cdot\left\{-2 b_{10} X^{3} r_{0}-2 b_{10} X Y^{2} r_{0}+2 X^{3} r_{0}^{2} \sqrt{X^{2}+Y^{2}}\right. \\
& +2 X Y^{2} r_{0}^{2} \sqrt{X^{2}+Y^{2}}+X Y^{2} \rho^{2} \sqrt{X^{2}+Y^{2}} \\
& -3 X^{3} Y \rho r_{0} \sin \left(\arctan \frac{X}{Y}+\gamma\right) \\
& -3 X Y^{3} \rho r_{0} \sin \left(\arctan \frac{X}{Y}+\gamma\right) \\
& -X^{2} Y^{2} \rho r_{0} \cos \left(\arctan \frac{X}{Y}+\gamma\right) \\
& \left.-Y^{4} \rho r_{0} \cos \left(\arctan \frac{X}{Y}+\gamma\right)\right\}
\end{aligned}
$$


where

$$
b_{10}=\sqrt{X^{2} r_{0}^{2}+Y^{2} r_{0}^{2}+Y^{2} \rho^{2}-2 Y \rho r_{0} \sqrt{X^{2}+Y^{2}} \sin \left(\arctan \frac{X}{Y}+\gamma\right)} .
$$

Next, the Taylor series coefficient $a_{10}$ is evaluated at the aperture center; that is; where $(X, Y)=\left(0, k_{0}\right)$. First, solving for $b_{10}$ :

$$
\begin{aligned}
\left.b_{10}\right|_{\substack{X=0, Y=k_{0}}}= & \left\{X^{2} r_{0}^{2}+Y^{2} r_{0}^{2}+Y^{2} \rho^{2}\right. \\
& \left.\quad-2 Y \rho r_{0} \sqrt{X^{2}+Y^{2}} \sin \left(\arctan \frac{X}{Y}+\gamma\right)\right\}\left.^{\frac{1}{2}}\right|_{\substack{X=0, Y=k_{0}}} \\
= & \sqrt{r_{0}^{2} k_{0}^{2}+\rho^{2} k_{0}^{2}-2 r_{0} k_{0}^{2} \rho \sin (\gamma)} \\
= & k_{0} \sqrt{r_{0}^{2}+\rho^{2}-2 \rho r_{0} \sin (\gamma)}
\end{aligned}
$$

and by substitution from Equation (4.52),

$$
\left.b_{10}\right|_{\substack{X=0, Y=k_{0}}}=r_{c} k_{0}
$$

Next, evaluating the linear phase coefficient $a_{10}$ given by Equation (4.56) at the aperture center yields

$$
\left.a_{10}\right|_{\substack{X=0, Y=k_{0}}}=\frac{1}{\left.b_{10}\right|_{\substack{X=0, Y=k_{0}}}} k_{0} \rho r_{0} \cos (\gamma)
$$

and by Equation (4.62),

$$
\begin{aligned}
\left.a_{10}\right|_{\substack{X=0, Y=k_{0}}} & =\frac{k_{0} \rho r_{0} \cos (\gamma)}{r_{c} k_{0}} \\
& =\frac{r_{0} \rho \cos (\gamma)}{r_{c}}
\end{aligned}
$$

Finally, from Equation (4.45),

$$
\left.a_{10}\right|_{\substack{X=0, Y=k_{0}}}=\frac{x_{0}^{\prime} r_{0}}{r_{c}}
$$


where, rewriting Equation (4.52) in terms of the target location $\left(x_{0}^{\prime}, y_{0}^{\prime}\right)$,

$$
\begin{aligned}
r_{c} & =\sqrt{r_{0}^{2}+\rho^{2}-2 \rho r_{0} \sin (\gamma)} \\
& =\sqrt{r_{0}^{2}+{x_{0}^{\prime}}^{2}+y_{0}^{\prime 2}-2 r_{0} y_{0}^{\prime}} .
\end{aligned}
$$

Thus, the linear cross-range coefficient $a_{10}$ is found to be:

$$
a_{10}=\frac{x_{0}^{\prime} r_{0}}{r_{c}}
$$

This Taylor coefficient, multiplied by the cross-range frequency extent $X^{\prime}$ as shown in Equation (4.12), determines the linear cross-range Fourier domain phase component of a point target at $\left(x_{0}^{\prime}, y_{0}^{\prime}\right)$. Consequently, upon Fourier transformation of the phase history, this phase component determines the spatial location in azimuth of the point in the imaged scene. That is, from Equation (4.12), the linear cross-range phase term $\phi_{1 t_{x}}$ is

$$
\begin{aligned}
\phi_{1 t_{x}} & =a_{10} X^{\prime} \\
& =\frac{x_{0}^{\prime} r_{0}}{r_{c}} X^{\prime},
\end{aligned}
$$

where $X^{\prime}$ is the Fourier frequency extent in cross-range. This phase term contains the linear phase component $x_{0}^{\prime} X^{\prime}$ that serves to correctly place the target in cross-range within the scene, and the phase error component $f_{\hat{x}}\left(x_{0}^{\prime}, y_{0}^{\prime}\right) X^{\prime}$, which is a consequence of the planar wavefront assumption and displaces the target from its correct position. This phase error component causes the cross-range geometric distortion that is induced by wavefront curvature.

As of yet, the value for linear phase given in Equation (4.70) has not been decomposed into its separate linear and error components. Specifically, it must be in the form $x_{0}^{\prime} X^{\prime}+f_{\hat{x}}\left(x_{0}^{\prime}, y_{0}^{\prime}\right) X^{\prime}$, where $f_{\hat{x}}\left(x_{0}^{\prime}, y_{0}^{\prime}\right) X^{\prime}$ specifies the cross-range distortion. In this way, the geometric distortion can be quantified and removed via a post-warping procedure. This decomposition will be discussed in Section 4.3.2. It should be noted here, however, that it is not trivial to represent Equation (4.70) in 
terms of its "correct" linear displacement and the additional distortion term. A reasonable approximation can be used to simplify this task, or alternatively, an elegant mathematical trick can be used to perform the decomposition, as will be presented in Chapter 5. The latter method is particularly attractive in determining the linear distortion terms for squint-mode geometries, which are more mathematically complicated than those for broadside. Phase errors for squint-mode collections will be discussed in Section 4.4.

An approach identical to the above is used to determine the linear range coefficient $a_{01}$, which will serve to place the point target in range. In this case, from Equation (4.48), $a_{01}$ is calculated as follows:

$$
\begin{aligned}
a_{01}= & \frac{\partial \phi_{x_{0}^{\prime}, y_{0}^{\prime}}\left(X^{\prime}, Y^{\prime}\right)}{\partial Y} \\
= & \frac{1}{b_{01} Y^{2}\left(X^{2}+Y^{2}\right)} \cdot\left\{b_{01} Y^{4} r_{0}-b_{01} X^{4} r_{0}-Y^{3} r_{0}^{2} \sqrt{X^{2}+Y^{2}}\right. \\
& +\frac{X^{4} r_{0}^{2}}{Y} \sqrt{X^{2}+Y^{2}}-Y^{3} \rho^{2} \sqrt{X^{2}+Y^{2}} \\
& -X^{4} \rho r_{0} \sin \left(\arctan \frac{X}{Y}+\gamma\right) \\
& -X^{3} Y \rho r_{0} \cos \left(\arctan \frac{X}{Y}+\gamma\right) \\
& +X^{2} Y^{2} \rho r_{0} \sin \left(\arctan \frac{X}{Y}+\gamma\right) \\
& -X Y^{3} \rho r_{0} \cos \left(\arctan \frac{X}{Y}+\gamma\right) \\
& \left.+2 Y^{4} \rho r_{0} \sin \left(\arctan \frac{X}{Y}+\gamma\right)\right\}
\end{aligned}
$$

where

$$
\begin{aligned}
b_{01} & =\frac{b_{10}}{Y} \\
& =\frac{1}{Y} \sqrt{X^{2} r_{0}^{2}+Y^{2} r_{0}^{2}+Y^{2} \rho^{2}-2 Y \rho r_{0} \sqrt{X^{2}+Y^{2}} \sin \left(\arctan \frac{X}{Y}+\gamma\right)} .
\end{aligned}
$$


Solving for $b_{01}$ at the aperture center $\left(0, k_{0}\right)$ :

$$
\begin{aligned}
\left.b_{01}\right|_{\substack{X=0, Y=k_{0}}}= & \frac{1}{Y}\left\{X^{2} r_{0}^{2}+Y^{2} r_{0}^{2}+Y^{2} \rho^{2}\right. \\
& \left.\quad-2 Y \rho r_{0} \sqrt{X^{2}+Y^{2}} \sin \left(\arctan \frac{X}{Y}+\gamma\right)\right\}\left.^{\frac{1}{2}}\right|_{\substack{X=0, Y=k_{0}}} \\
= & \frac{1}{k_{0}} \sqrt{r_{0}^{2} k_{0}^{2}+\rho^{2} k_{0}^{2}-2 r_{0} k_{0}^{2} \rho \sin (\gamma)} \\
= & \sqrt{r_{0}^{2}+\rho^{2}-2 \rho r_{0} \sin (\gamma)},
\end{aligned}
$$

and by substitution from Equation (4.52),

$$
\left.b_{01}\right|_{\substack{X=0, Y=k_{0}}}=r_{c} .
$$

Having determined $b_{01}$ at aperture center, the linear phase coefficient $a_{01}$ given by Equation (4.71) can also be evaluated at the aperture center, as follows:

$$
\left.a_{01}\right|_{\substack{X=0, Y=k_{0}}}=r_{0}-\frac{1}{\left.b_{01}\right|_{\substack{X=0, Y=k_{0}}}}\left(r_{0}^{2}-\rho^{2}-2 \rho r_{0} \sin (\gamma)\right)
$$

and by Equation (4.78),

$$
\left.a_{01}\right|_{\substack{X=0, Y=k_{0}}}=r_{0}-\frac{r_{0}^{2}-\rho^{2}-2 \rho r_{0} \sin (\gamma)}{r_{c}}
$$

Finally, again by Equation (4.52),

$$
\left.a_{01}\right|_{\substack{X=0, Y=k_{0}}}=r_{0}-\frac{r_{c}^{2}}{r_{c}}=r_{0}-r_{c},
$$

where, as previously shown,

$$
r_{c}=\sqrt{r_{0}^{2}+x_{0}^{\prime 2}+y_{0}^{\prime 2}-2 r_{0} y_{0}^{\prime}}
$$

for the slant plane target position $\left(x_{0}^{\prime}, y_{0}^{\prime}\right)$ and range $r_{0}$ from the scene center to radar platform at mid-aperture. The Taylor coefficient $a_{01}$, when multiplied by the 
range frequency extent $Y^{\prime}$, determines the linear phase component in range of a point target at $\left(x_{0}^{\prime}, y_{0}^{\prime}\right)$. That is, from Equation (4.12), the linear range phase term $\phi_{1 t_{y}}$ is

$$
\begin{aligned}
\phi_{1 t_{y}} & =a_{01} Y^{\prime} \\
& =\left(r_{0}-r_{c}\right) Y^{\prime},
\end{aligned}
$$

where $Y^{\prime}$ is the Fourier frequency extent in range. As with the linear cross-range component, this phase term contains the linear phase component $y_{0}^{\prime} Y^{\prime}$ that serves to correctly place the target in range within the scene, as wrell as the phase error component $f_{\hat{x}^{\prime}} Y^{\prime}$, which is a consequence of the planar wavefront assumption and displaces (geometrically distorts) the target from its correct position. In the next section, the linear range and cross-range phase terms will be broken into their components, identifying the specific linear phase error terms which induce the wavefront curvature distortion.

\subsubsection{Decomposition of Linear Phase Terms}

The linear phase terms of a point target constitute the two-dimensional complex sinusoid $e^{j \phi_{1 t}}$ which determines the spatial placement of the point in the imaged scene. Ideally, of course, in the absence of significant wavefront curvature, the linear phase $\phi_{1 t}$ is specified by Equation (4.8) to be $x_{0}^{\prime} X^{\prime}+y_{0}^{\prime} Y^{\prime}$. That is, the displacement in cross-range and range is proportional to the linear phase in those directions. In the previous discussion, a Taylor series expansion was performed on the equation describing the phase return from a point target with respect to the linear (firstorder) components. The the sum of these phase terms $\phi_{1 t}$ was found to be

$$
\phi_{1 t}=\frac{x_{0}^{\prime} r_{0}}{r_{c}} X^{\prime}+\left(r_{0}-r_{c}\right) Y^{\prime}
$$

While these terms completely describe the position of the target in the imaged scene, they are not yet a form which describes the target in terms of its correct position in the scene, and its displacement in the presence of wavefront curvature. In describing 
the linear phase components in this new way, the specific geometric distortion can be quantified. That is, one can determine (and ultimately correct, as will be shown) the erroneous displacement of a point target from its correct position due to wavefront curvature. Thus, the desired form for the linear phase terms, as given by Equation (4.13), is

$$
\phi_{1 t}=x_{0}^{\prime} X^{\prime}+y_{0}^{\prime} Y^{\prime}+f_{\hat{x}^{\prime}} X^{\prime}+f_{\hat{y}^{\prime}} Y^{\prime}
$$

where $f_{\hat{x}^{\prime}} X^{\prime}$ and $f_{\hat{y}^{\prime}} Y^{\prime}$ denote the cross-range and range distortion terms, respectively.

When the scene size is much smaller than the slant range at mid-aperture; that is, when $x_{0}^{\prime} \ll r_{0}$ and $y_{0}^{\prime} \ll r_{0}$ (small patch assumption), then from Figure 4.3, $y_{0}^{\prime}=\rho \sin (\gamma) \approx r_{0}-r_{c}$. Consequently, the linear phase terms can be decomposed as follows. From Equation (4.84), consider the cross-range linear term first:

$$
\begin{aligned}
\phi_{1 t_{x}} & =\frac{x_{0}^{\prime} r_{0}}{r_{c}} X^{\prime} \\
& \approx \frac{x_{0}^{\prime} r_{0}}{r_{0}-y_{0}^{\prime}} X^{\prime} \\
& \approx \frac{x_{0}^{\prime}\left(r_{0}+y_{0}^{\prime}\right)}{\left(r_{0}+y_{0}^{\prime}\right)-y_{0}^{\prime}} X^{\prime} \\
& =\left\{\frac{x_{0}^{\prime} r_{0}}{r_{0}}+\frac{x_{0}^{\prime} y_{0}^{\prime}}{r_{0}}\right\} X^{\prime} \\
& =x_{0}^{\prime} X^{\prime}+\frac{x_{0}^{\prime} y_{0}^{\prime}}{r_{0}} X^{\prime}, \quad x_{0}^{\prime} \ll r_{0}, y_{0}^{\prime} \ll r_{0}
\end{aligned}
$$

and furthermore, given the Taylor series approximation

$$
\begin{aligned}
r_{c} & =\sqrt{r_{0}^{2}+\rho^{2}-2 \rho r_{0} \sin (\gamma)} \\
& \approx r_{0}-y_{0}^{\prime}+\frac{x_{0}^{\prime 2}}{2 r_{0}},
\end{aligned}
$$

then for the linear term in range,

$$
\begin{aligned}
\phi_{1 t_{y}} & =\left(r_{0}-r_{c}\right) Y^{\prime} \\
& \approx y_{0}^{\prime} Y^{\prime}-\frac{x_{0}^{\prime}}{2 r_{0}} Y^{\prime} .
\end{aligned}
$$


Thus, by Equation (4.13),

$$
\begin{aligned}
\phi_{1 t} & =x_{0}^{\prime} X^{\prime}+y_{0}^{\prime} Y^{\prime}+f_{\hat{x}^{\prime}} X^{\prime}+f_{\hat{y}^{\prime}} Y^{\prime} \\
& \approx x_{0}^{\prime} X^{\prime}+y_{0}^{\prime} Y^{\prime}+\frac{x_{0}^{\prime} y_{0}^{\prime}}{r_{0}} X^{\prime}-\frac{x_{0}^{\prime 2}}{2 r_{0}} Y^{\prime}, \quad x_{0}^{\prime}, y_{0}^{\prime} \ll r_{0},
\end{aligned}
$$

where $\frac{x_{0}^{\prime} y_{0}^{\prime}}{r_{0}} X^{\prime}$ and $-\frac{x_{0}^{\prime 2}}{2 \tau_{0}} Y^{\prime}$ denote the cross-range and range geometric distortion (error) phase terms $f_{\hat{x}^{\prime}} X^{\prime}$ and $f_{\hat{y}^{\prime}} Y^{\prime}$, respectively. Note that these terms (and consequently the distortion) decrease with increasing slant range $r_{0}$, as expected, and that this distortion is independent of radar wavelength $\lambda$. Equation (4.88) is most accurate under the small patch assumption $x_{0}^{\prime}, y_{0}^{\prime} \ll r_{0}$, which ironically, is when geometric distortion is minimal anyway. However, this approximation allows the expression of linear phase as the sum of terms which represent the proper placement of the target and the space-variant distortion terms which displace it. In the absence of significant wavefront curvature, the error terms are (nearly) zero and the linear phase specifies the proper point target location of $\left(x_{0}^{\prime}, y_{0}^{\prime}\right)$. In the presence of wavefront curvature, the error terms specify the displacement in range and azimuth of a point that would otherwise be placed at $\left(x_{0}^{\prime}, y_{0}^{\prime}\right)$. By the linear shift property of the Fourier transform, the linear phase error coefficients $f_{\hat{x}^{\prime}}$ and $f_{\hat{y}^{\prime}}$, when expressed in meters, relate directly to target translations, in meters. Consequently, these phase error coefficients are used to generate the appropriate warping function for the post-processing procedure to correct for image distortions due to wavefront curvature. Thus, in addition to being independent of radar wavelength, the geometric distortion is also independent of radar resolution, and therefore relies only on patch size and standoff range $r_{0}$. The application of the space-variant post-filter for image refocusing also requires knowing the proper undistorted location of targets in the imaged scene. As will be shown in Chapter 5, an elegant mathematical trick, as opposed to the small patch approximation given here, will be applied to precisely determine the geometrically correct target points, with no restrictions on standoff range or patch size.

In Chapter 3 , the geometric distortion in range $\Delta y^{\prime}$, or range sag, was derived 
from a geometric model that represented the physical characteristics of spherical wavefronts. The range sag equation is given again here for convenience.

$$
\Delta y^{\prime}=\frac{L^{2}}{2 r_{0}}
$$

where $L$ is the patch radius at the edge of the scene, and the approximation $r_{0} \gg L$ is applied. Consequently, the angle $\theta_{L}$ from aperture center to scene edge is small. These assumptions of the physical model exactly parallel the small patch approximation of this chapter. Similarly, there is a direct parallel between the range sag equation of Chapter 3 (Equation (4.89)) and the phase analysis model discussed here, which represents geometric distortion in terms of linear phase errors. Specifically, for range distortion,

$$
\Delta y^{\prime}=\frac{L^{2}}{2 r_{0}}, \quad L \ll r_{0} \quad \text { (geometric model) }
$$

and

$$
f_{\hat{y}^{\prime}}=\frac{x_{0}^{\prime 2}}{2 r_{0}}, \quad x_{0}^{\prime}, y_{0}^{\prime} \ll r_{0} . \quad \text { (phase model) }
$$

Since the models are equivalent when $L=x_{0}^{\prime}$ and $y_{0}^{\prime}=0$; that is, when considering range distortion at the edge of the patch, then

$$
\Delta y^{\prime}=f_{\tilde{y}^{\prime}}
$$

and the models are in perfect agreement. A similar argument can be made for $\Delta x^{\prime}$ and $f_{\hat{x}^{\prime}}$.

The linear Taylor series terms dominate those of higher order. Consequently, geometric distortion is the prominent and first observable indication of wavefront curvature. As wavefront curvature becomes more severe and higher-order phase terms become significant, a space-variant defocus begins to emerge, along with the increasing geometric distortion. This distortion is prominent even when wavefront curvature is minimal, and consequently, the post-warping, geometric correction step of PFA has always been a standard part of the algorithm. The space-variant defocus 
effects induced by higher-order phase errors are observed only in the more severe cases of wavefront curvature. However, these defocus effects are significantly more troublesome to negate than those of geometric distortion.

\subsubsection{Quadratic Phase Terms}

The quadratic phase terms are the next to be derived from the Taylor series expansion. As previously shown, the linear phase consists of terms that correctly place the point in the image domain as well as extraneous linear distortion terms. In contrast, any quadratic phase that is present serves only to degrade the image by way of space-variant defocus. There is no "good" quadratic phase. From Equation (4.15), the quadratic phase error $\phi_{2 t}$ is described in terms of its components and is defined to be

$$
\phi_{2 t}=f_{\widehat{x^{2}}} X^{\prime 2}+f_{\widehat{x y^{\prime}}} X^{\prime} Y^{\prime}+f_{\widehat{y}^{2}} Y^{\prime 2}
$$

In terms of the Taylor series expansion, the quadratic phase is equivalently expressed as

$$
\phi_{2 t}=a_{20} X^{\prime 2}+a_{11} X^{\prime} Y^{\prime}+a_{02} Y^{\prime 2}
$$

where the coefficients are based on Equation (4.48) and found to be

$$
\begin{aligned}
& a_{20}=\left.\left(\frac{1}{2}\right)\left(\frac{\partial^{2} k\left(r_{x}-r_{t}\right)}{\partial X^{2}}\right)\right|_{\substack{X=X_{0}, Y=Y_{0}}}, \\
& a_{11}=\left.\left(\frac{\partial^{2} k\left(r_{x}-r_{t}\right)}{\partial X \partial Y}\right)\right|_{\substack{X=X_{0}, Y=Y_{0},}},
\end{aligned}
$$

and

$$
a_{02}=\left.\left(\frac{1}{2}\right)\left(\frac{\partial^{2} k\left(r_{x}-r_{t}\right)}{\partial Y^{2}}\right)\right|_{\substack{X=X_{0}, Y=Y_{0}}},
$$

given the slant plane, broadside-only phase return from a point target $\phi_{x_{0}^{\prime}, y_{0}^{\prime}}\left(X^{\prime}, Y^{\prime}\right)=$ $k\left(r_{x}-r_{t}\right)$ (Equation (4.44)). It will be shown in Section (4.4) that the range-only 
and range-azimuth quadratic terms $a_{11} X^{\prime} Y^{\prime}$ and $a_{02} Y^{\prime 2}$, respectively, are zero for all slant-ranges, squint angles, radar wavelengths, patch sizes and resolutions. That is, the quadratic defocus is one-dimensional in cross-range only, regardless of the imaging scenario or squint mode. This is one of the most important results of this dissertation, as one-dimensional filtering is more computationally efficient that twodimensional. Furthermore, it will be shown the broadside case described thus far is simply a specific case of the generalized squint mode scenario, where the squint angle $\theta_{s}$ happens to be zero. Given that quadratic phase error is one-dimensional is cross-range only, Equation (4.92) reduces to the following description of quadratic phase:

$$
\phi_{2 t}=a_{20} X^{\prime 2}, \quad a_{11} X^{\prime} Y^{\prime}=0, a_{02} Y^{\prime 2}=0 .
$$

From Equations (4.44) and (4.93), the quadratic phase coefficient in cross-range is calculated as follows, when evaluated at the aperture center $(X, Y)=\left(0, k_{0}\right)$ :

$$
\begin{aligned}
a_{20} & =\left.\left(\frac{1}{2}\right)\left(\frac{\partial^{2} k\left(r_{x}-r_{t}\right)}{\partial X^{2}}\right)\right|_{\substack{X=0, Y=k_{0}}} \\
& =\frac{1}{2}\left\{\frac{r_{0}-r_{c}}{k_{0}}+\frac{r_{0}}{k_{0}}+\frac{x_{0}^{\prime 2} r_{0}^{2}}{r_{c}^{3} k_{0}}-\frac{r_{0}^{2}}{r_{c} k_{0}}\right\} \\
& =\frac{1}{2}\left\{\frac{r_{0}-r_{c}}{k_{0}}-\frac{r_{0}\left(r_{0}-r_{c}\right)}{r_{c} k_{0}}+\frac{x_{0}^{\prime 2} r_{0}^{2}}{r_{c}^{3} k_{0}}\right\},
\end{aligned}
$$

where $k_{0}=\frac{4 \pi}{\lambda}$ is the nominal phase history radius, $r_{0}$ is the slant range at broadside, and from Figure 4.3, the distance $r_{c}$ from the platform at mid-aperture to the target at $\left(x_{0}^{\prime}, y_{0}^{\prime}\right)$ is

$$
\begin{aligned}
r_{c} & =\sqrt{r_{0}^{2}+\rho^{2}-2 \rho r_{0} \sin (\gamma)} \\
& =\sqrt{r_{0}^{2}+x_{0}^{\prime 2}+y_{0}^{\prime 2}-2 r_{0} y_{0}^{\prime}} .
\end{aligned}
$$

Finally, from Equations (4.96) and (4.97), the total quadratic phase for a broadside collection is found to be

$$
\begin{aligned}
\phi_{2 t} & =a_{20}{X^{\prime 2}}^{2} \\
& =\frac{1}{2}\left\{\frac{r_{0}-r_{c}}{k_{0}}-\frac{r_{0}\left(r_{0}-r_{c}\right)}{r_{c} k_{0}}+\frac{x_{0}^{\prime 2} r_{0}^{2}}{r_{c}^{3} k_{0}}\right\} X^{\prime 2},
\end{aligned}
$$


where $X^{\prime 2}$ is the spatial frequency extent in cross-range and the maximum value of $X^{\prime}$ is equal to $\frac{\pi}{\rho_{x}}$ (the half-power width), given a cross-range resolution of $\rho_{x}$.

The relation of Equation (4.98) describes the total quadratic phase for a broadside collection, without introducing approximations. Thus, it applies to all patch sizes at all resolutions and slant ranges. It is a closed-form, deterministic measure of quadratic phase that is based on the specific imaging geometry and the point of interest in the scene. As shown above, this equation hardly resembles (in terms of simplicity) that of Equation (3.5), which described the quadratic phase error given the small patch assumption, where $x_{0}^{\prime}, y_{0}^{\prime} \ll r_{0}$. However, by applying the approximations associated with the small patch assumption, as was done in Section 4.3.2 for the linear phase terms, in addition to the Taylor series approximation $r_{c} \approx$ $r_{0}-y_{0}^{\prime}+\frac{1}{2} \frac{x_{0}^{\prime 2}}{r_{0}}$, Equation (4.98) can be approximated by Equation (3.5). Specifically,

$$
\begin{aligned}
\phi_{2 t} & =\frac{1}{2}\left\{\frac{r_{0}-r_{c}}{k_{0}}-\frac{r_{0}\left(r_{0}-r_{c}\right)}{r_{c} k_{0}}+\frac{x_{0}^{\prime 2} r_{0}^{\prime 2}}{r_{c}^{3} k_{0}}\right\} X^{\prime 2} \\
& \approx \frac{x_{0}^{\prime 2}-y_{0}^{\prime 2}}{2\left(r_{0}-y_{0}\right) k_{0}} X^{\prime 2} \\
& \approx \frac{x_{0}^{\prime 2}-y_{0}^{\prime 2}}{2 r_{0} k_{0}} X^{\prime 2}, \quad x_{0}^{\prime} \ll r_{0}, y_{0}^{\prime} \ll r_{0} .
\end{aligned}
$$

It is evident from Equation (4.100) that the quadratic phase error $\phi_{2 t}$ increases with better resolution (increasing $X^{\prime 2}$ ), decreasing slant range $r_{0}$, lower center frequency (decreasing $k_{0}$ ) and larger imaged patches (increasing $x_{0}^{\prime}$ and $y_{0}^{\prime}$ ). Furthermore, as was demonstrated in Chapter 3 , defocus is space-variant and greater along the $x^{\prime}$ and $y^{\prime}$ axes and minimized along the diagonals given by $y^{\prime}=\left|x^{\prime}\right|$. Equation (4.99) is a slightly better approximation than that of (4.100). According to these approximations, the quadratic phase error is exactly zero along the diagonals. However, when considering the exact relation of Equation (4.98), the phase error is nearly zero along the diagonals, but exactly zero only at the precise center of the patch. Given the small patch approximations, Equation (4.99) is equivalent to that of (3.5), which was derived via an alternative method in [7, pp. 361-363], and yet again in [12, pp. 220-234]. These consistencies are important (and in fact necessary) 
in order to validate the geometric model presented here. Next, this model will be extended to consider squinted collections, thereby removing the broadside restriction and generalizing space-variant post-filtering to all spotlight-mode imaging scenarios.

\subsection{Generalized Phase Error Model for all Squint Modes}

The previous discussion considers only the broadside-mode spotlight imaging scenario. While this is the most fundamental case and the easiest to model and analyze, it is not suitable for most real world imaging scenarios since these are usually squinted collections. Consequently, without the ability to compensate wavefront curvature in squinted modes, the space-variant post-filter is little more than an academic exercise. Given the theoretical nature of this SAR research, this is not a negative. However, by generalizing the geometric model to cover squint-mode scenarios, the space-variant post-filter extends beyond the theoretical bounds. In doing so, the contribution of this research is of much greater significance. In fact, the major contribution of this dissertation is the generalized, squint-mode phase error equations to be introduced next, which serve to compensate wavefront curvature errors in all squint modes, and at all ranges for all patch sizes. Also of major significance are the computer implementation details of Chapter 5 , which provide an effective and efficient means of applying these equations to compensate the effects of wavefront curvature in polar-formatted, spotlight-mode images.

\subsubsection{Squint-Mode Geometry}

Squint-mode imaging consists of "looking backwards or forwards" at the scene being imaged. As with the broadside case, the radar antenna is slewed as the aperture is flown so as to stay aimed at the scene center. However, unlike the broadside 
case, the scene being imaged is not at a right angle (perfectly side-looking) to the radar platform at aperture center. Consequently, the image is motion compensated to the scene center, yet the motion of this point (range curvature of the CRP) is not symmetric relative to the midpoint of the aperture. Within the tomographic paradigm, this means the generation of a Fourier slant plane that is tilted in both range and cross-range, due to the difference in starting depression angle $\psi_{o}$ relative to the ending depression angle $\psi_{f}$ at the end of the aperture. This results in the projection of ground plane targets into the slant plane such that the ground scene is distorted in both range and cross-range when viewed in the slant plane. However, as did the previous discussion, this section considers the slant plane Fourier space without regard to the geometric projection of points into this plane from the ground. As such, the wavefront curvature model, phase derivation, distortion correction and refocus techniques will apply strictly to the slant-plane imagery.

It is important to note that the squint-mode case is not simply a rotated version of the broadside case, since the CRP movement is not symmetric relative to the aperture center. Thus, the asymmetric motion must be appropriately accounted for in the squint-mode model. As will be shown, this model is significantly more complicated than that of the broadside scenario, yet the mathematical reductions yield relations that are nearly as elegant. Perhaps the most pleasant surprise is that space-variant defocus remains one-dimensional in the cross range direction, even for squinted collections, thereby providing the segue for efficient computer implementations. One should also note that the broadside-mode scenario previously discussed is simply a special case of squint-mode, where the squint angle $\theta_{s}$ happens to be zero. Thus, as would be expected, it will be shown that the new, generalized geometric model "collapses" to that of the previous, simpler broadside model when the squint angle is zero. Similarly, the relations derived which represent the linear and quadratic phase errors also reduce to the previous broadside-mode phase equations when the squint angle is zero.

Prior to introducing the model representing the phase return from a point target 


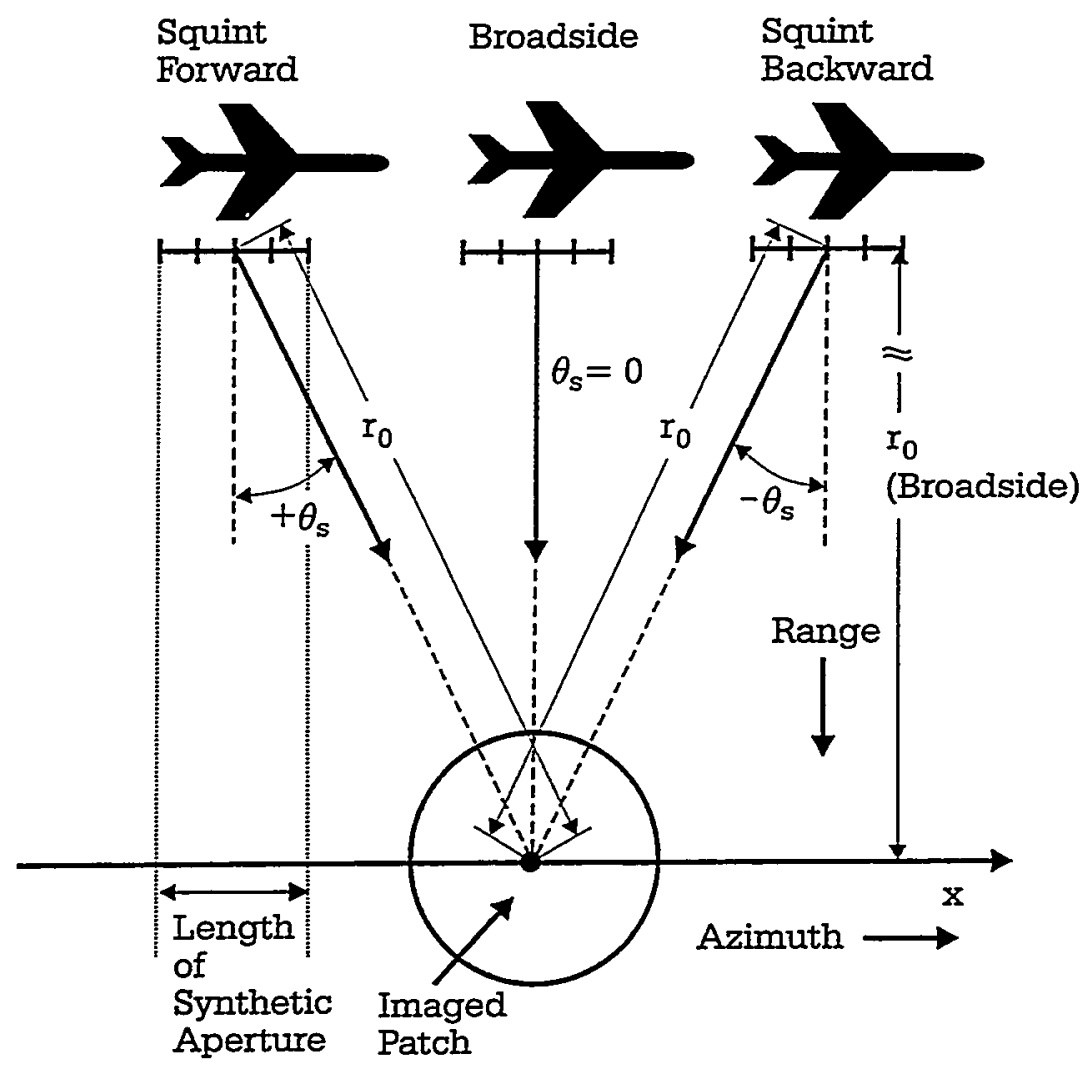

Figure 4.4: Squint Angle and Standoff Range Definitions for Spotlight-Mode

in squint-mode, the coordinate system and polarity of the squint must be defined. As shown in Figure 4.4, when the radar platform is looking forward towards the scene, this is considered positive squint, or $+\theta_{s}$. When looking backwards (behind the shoulder), this is negative squint, $-\theta_{s}$. If the platform is traveling in the direction opposite to that shown, then what was previously considered "looking backward" is now the opposite, and it is a simple matter of reversing the sign of the squint value to compensate. Regarding the coordinate system, the traditional perspective has been to align the $X^{\prime}$ axis (of both the imaged scene and the phase history) with the flight path so they are parallel. This axial orientation is also shown in Figure 4.4.

The geometric model for the phase return from a point target is shown in Figure 4.5, and applies to the generalized squint-mode case. This model is an extension of the broadside model of Figure 4.2, and their similarities are apparent. Part (a) 
represents the traditional view of the squinted collection, while part (c) is the corresponding Fourier space. Both are shown with the flight path parallel to the $X^{\prime}$ axis. In the squinted case, the mid-aperture point is no longer aligned on the $Y^{\prime}$ axis, as shown in Figure 4.2, but instead at some angular offset $\theta_{s}$ from the $Y^{\prime}$ axis. Consequently, the Fourier space is "rotated" the same amount, with the center of the frequency extent also found at an angle of $\theta_{s}$. When the squint angle is zero (broadside collection), it is easily seen that parts (a) and (c) are in agreement with Figure 4.2. That is, the generalized squint geometry is equivalent to that of the broadside case when the squint angle is zero.

Unfortunately, the traditional view of the squinted scenario is not the appropriate perspective if a computationally efficient model is to be derived for wavefront curvature correction. Specifically, the rotation of the phase history, as shown in part (c), is relative to the arbitrary, Cartesian axis of reference and is not in agreement with the orientation of the SAR platform collecting the data. In fact, the squinted Fourier data are actually acquired and stored as shown in Figure 4.5 (d); that is, with respect to the platform, which slews toward the scene center as the aperture is flown. The actual range axis lies along the line defined by the CRP and the platform at mid-aperture, which is consistent with the simplified, broadside model of the previous section. Using the traditional view would require the costly (and unnecessary) rotation of the entire phase history by an amount of $\theta_{s}$, in order be consistent with the flight path as shown in part (a). Consequently, the modified flight path perspective shown in Figure 4.5 (b) is offered as an equivalent alternative to the traditional view, and it does not require the global rotation of the Fourier phase history data.

While the imaging geometry of Figure 4.5 (b) is seemingly more complicated than that of part (a), this modified view allows the use of the Fourier phase history in-situ, without a costly global rotation and the interpolations associated with it. As will be shown next, the geometry associated with the modified view in part (b) does lead to some cumbersome mathematics; however, the simplified phase error equations are elegant, straightforward to implement and computationally efficient, 

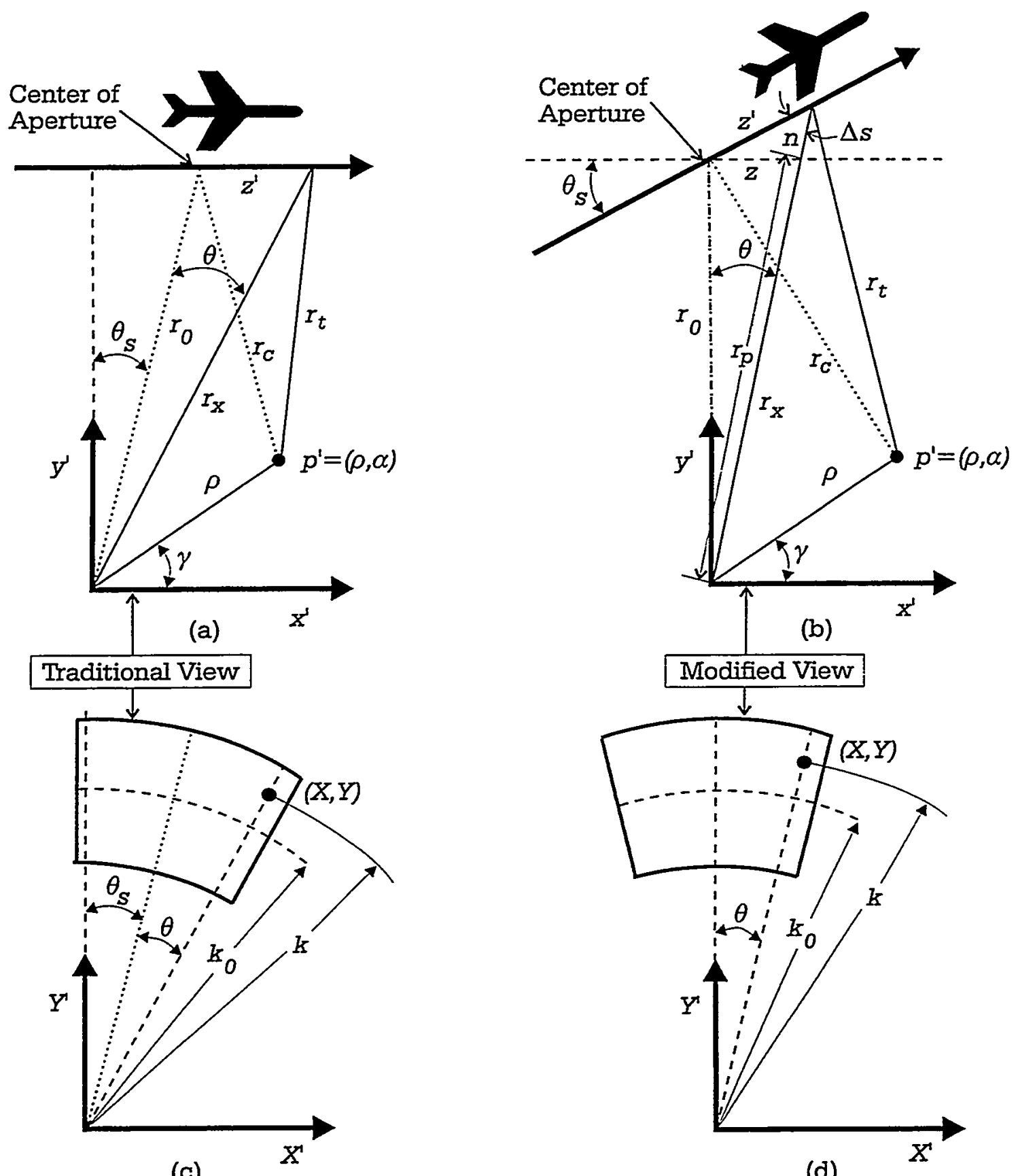

Figure 4.5: Geometric Model for Derivation of Squinted-Collection Wavefront Curvature Phase Errors

even in their exact form without approximations. Thus, what was once thought to be a complicating transformation; that is, the selection of the modified perspective 
for the squint-mode model, in fact turns out to be its saving grace. Again, when considering the modified view of parts (b) and (d), it is clear that when the squint angle $\theta_{s}$ is zero, then the flight path becomes parallel to the $X^{\prime}$ axis, $r_{p}=r_{x}$, and the model is identical to that of the previous broadside scenario.

A procedure identical to that of the broadside case will now be used to derive the phase error equations for the generalized squint-mode scenario. Specifically, the phase return of a point target must be determined in Fourier space based on its spatial $\left(x_{0}^{\prime}, y_{0}^{\prime}\right)$ position in the imaged scene and the relative platform position. Based on a-priori knowledge that the platform trajectory is linear (as depicted in the model), as well as the subsequent evaluation of the Taylor terms at the spectral center of the processing aperture, a closed-form solution for the linear and quadratic phase error terms can be found strictly as a function of the scene position, without regard to individual aperture positions along the flight path. This approach lends itself to efficient computer implementations. Thus, as with the previous broadside model, it is desirable to express the Fourier phase history domain $\left(X^{\prime}, Y^{\prime}\right)$ in terms of a hypothetical point target $\left(x_{0}^{\prime}, y_{0}^{\prime}\right)$. That is, to represent the phase in the form of $\phi_{x_{0}^{\prime}, y_{0}^{\prime}}\left(X^{\prime}, Y^{\prime}\right)$. Again, this requires the further coupling (relation) of the spatial position and phase models for the squinted case, as shown in Figures 4.5 (b) and (d), respectively. This model is an extension of the previous broadside scenario, and consequently, several of the broadside relations are still applicable and are restated here for convenience. From Section 4.2, but now referring to Figures 4.5 (b) and (d),

$$
\phi_{x_{0}^{\prime}, y_{0}^{\prime}}\left(X^{\prime}, Y^{\prime}\right)=k\left(r_{x}-r_{t}\right)
$$

where

$$
k=\left[\frac{2 \omega_{0}}{c}+\frac{4 \alpha}{c}\left(t-\frac{2 r_{x}}{c}\right)\right] .
$$

Also, reflecting a minor change in symbols, Equation (4.33) becomes

$$
z=\frac{r_{0} X}{Y}, \quad r_{0}>0, Y>0
$$


and (4.37) becomes

$$
r_{p}=r_{0} \sqrt{1+\left(\frac{X}{Y}\right)^{2}}
$$

Additionally,

$$
r_{t}=\sqrt{r_{x}^{2}+\rho^{2}-2 \rho r_{x} \sin \left(\arctan \left(\frac{X}{Y}\right)+\gamma\right)} \quad \text { (Equation (4.42)) }
$$

Furthermore, the Cartesian slant plane representation $\left(x_{0}, y_{0}\right)$ of the point target $p=(\rho, \gamma)$ is given by

$$
x_{0}^{\prime}=\rho \cos \gamma
$$

and

$$
y_{0}^{\prime}=\rho \sin \gamma .
$$

Now, additional trigonometric relations are required to fully describe the new model in terms of its Fourier phase space, with respect to the target position in the imaged scene. These relations are specific to the squint-mode model of Figure 4.5 and are described as follows.

$$
\cos \theta=\frac{r_{0}}{r_{p}},
$$

and from Equation (4.102),

$$
\begin{aligned}
\cos \theta & =\frac{r_{0}}{r_{0} \sqrt{1+\left(\frac{X}{Y}\right)^{2}}} \\
& =\frac{1}{\sqrt{1+\left(\frac{X}{Y}\right)^{2}}} .
\end{aligned}
$$

Similarly,

$$
\sin \theta=\frac{z}{r_{p}},
$$


and from Equations (4.101) and (4.102),

$$
\begin{aligned}
\sin \theta & =\frac{r_{0} \frac{X}{Y}}{r_{0} \sqrt{1+\left(\frac{X}{Y}\right)^{2}}} \\
& =\frac{\frac{X}{Y}}{\sqrt{1+\left(\frac{X}{Y}\right)^{2}}} .
\end{aligned}
$$

The distance $r_{x}$ is defined as the sum of $n$ and $r_{p}$ in the squinted model:

$$
r_{x}=r_{p}+n \text {. }
$$

When the squint angle $\theta_{s}$ is zero, $n=0, r_{p}=r_{x}$ and $z^{t}=z$, which is consistent with the broadside model. In the case of nonzero squint, a new, irregular (non-right) triangle is defined by the edges $z, z^{\prime}$ and $n$. In this case, the angle $\Delta s$ is found to be

$$
\begin{aligned}
\Delta s & =90^{\circ}-\left(\theta_{s}+\theta\right) \\
& =90^{\circ}-\theta_{s}-\theta,
\end{aligned}
$$

and by the law of sines,

$$
\begin{aligned}
\frac{\sin \theta_{s}}{n} & =\frac{\sin \Delta s}{z} \\
& =\frac{\sin \left(90^{\circ}-\theta_{s}-\theta\right)}{z} .
\end{aligned}
$$

It is desired to represent the distance $n$ strictly in terms of the angle subtended at mid-aperture, $\theta$, the squint angle $\theta_{s}$, and the point in Fourier space, $(X, Y)$. In this way, the phase error can eventually be specified by the position in the image $\left(x_{0}^{\prime}, y_{0}^{\prime}\right)$, as was the case with the broadside-only collection, in addition to the new squint angle parameter, $\theta_{s}$. To this end, reducing Equation (4.110),

$$
\begin{aligned}
\frac{\sin \theta_{s}}{n} & =\frac{\sin \left(90^{\circ}\right) \cos \left(\theta_{s}+\theta\right)-\cos \left(90^{\circ}\right) \sin \left(\theta_{s}+\theta\right)}{z} \\
& =\frac{\cos \left(\theta_{s}+\theta\right)}{z} .
\end{aligned}
$$

It follows that

$$
\begin{aligned}
n & =\frac{z \sin \theta_{s}}{\cos \left(\theta_{s}+\theta\right)} \\
& =\frac{z \sin \theta_{s}}{\cos \theta_{s} \cos \theta-\sin \theta_{s} \sin \theta},
\end{aligned}
$$


and by substitution from Equations (4.101), (4.104) and (4.106),

$$
\begin{aligned}
n & =\frac{\frac{r_{0} X}{Y} \sin \theta_{s}}{\cos \theta_{s} \frac{1}{\sqrt{1+\left(\frac{X}{Y}\right)^{2}}}-\sin \theta_{s} \frac{X}{\sqrt{Y}} \sqrt{1+\left(\frac{X}{Y}\right)^{2}}} \\
& =\frac{\frac{1}{Y} \sin \theta_{s}}{\frac{1}{\sqrt{1+\left(\frac{X}{Y}\right)^{2}}}\left(\cos \theta_{s}-\frac{X}{Y} \sin \theta_{s}\right)} \\
& =\frac{r_{0} \sqrt{1+\left(\frac{X}{Y}\right)^{2}} \frac{X}{Y} \sin \theta_{s}}{\cos \theta_{s}-\frac{X}{Y} \sin \theta_{s}} .
\end{aligned}
$$

Now, by substitution from Equation (4.102),

$$
n=\frac{\frac{r_{p} X}{Y} \sin \theta_{s}}{\cos \theta_{s}-\frac{X}{Y} \sin \theta_{s}} .
$$

Having expressed $n$ in terms of the appropriate parameters, all that remains is to define the distance $r_{x}$ from platform to scene center (CRP) in terms of these same parameters $\theta, \theta_{s}$ and $(X, Y)$. Recalling Equation (4.107),

$$
r_{x}=r_{p}+n
$$

By substitution from Equation (4.115),

$$
\begin{aligned}
r_{x} & =r_{p}+\frac{\frac{r_{p} X}{Y} \sin \theta_{s}}{\cos \theta_{s}-\frac{X}{Y} \sin \theta_{s}} \\
& =r_{p}\left(1+\frac{\frac{X}{Y} \sin \theta_{s}}{\cos \theta_{s}-\frac{X}{Y} \sin \theta_{s}}\right)
\end{aligned}
$$

where

$$
r_{p}=r_{0} \sqrt{1+\left(\frac{X}{Y}\right)^{2}}
$$

For simplicity, let

$$
\Omega_{s}=\frac{\frac{X}{Y} \sin \theta_{s}}{\cos \theta_{s}-\frac{X}{Y} \sin \theta_{s}}, \quad-90^{\circ}<\theta_{s}<90^{\circ} .
$$

Then, from Equation (4.116),

$$
r_{x}=r_{p}\left(1+\Omega_{s}\right) .
$$


Having described $k, r_{t}$ and $r_{x}$ in terms of a specified point target for squint-mode scenarios (Equations (4.27), (4.42) and (4.118), respectively), the phase return from a point target $\phi_{x_{0}^{\prime}, y_{0}^{\prime}}\left(X^{\prime}, Y^{\prime}\right)=k\left(r_{x}-r_{t}\right)$ has been fully specified. Consequently, the Taylor series expansion can now be applied to find the linear and quadratic phase error terms. First, however, it is important to verify that the generalized squintmode model reduces to that of the specific broadside model under the appropriate circumstances. From Figures 4.5 (b) and (d), note that when the platform is at mid-aperture; that is, when $\theta=0$, then $X=0, r_{p}=r_{0}$, and $\Omega_{s}=0$ (from Equation (4.117)). Therefore, $r_{x}=r_{0}$ for all squint angles $\theta_{s}$ at mid-aperture. Consequently, $r_{c}=\sqrt{r_{0}^{2}+\rho^{2}-2 \rho r_{0} \sin \gamma}$ at mid-aperture, which is consistent with the broadside model of Figure 4.2 and Equation (4.52) for $r_{c}$.

Furthermore, the squint-mode model must reduce to the specific broadside case when the squint angle $\theta_{s}$ is zero. In this case, when $\theta_{s}=0$, then $n=0$ from Equation (4.115), and $r_{x}=r_{p}=r_{0} \sqrt{1+\left(\frac{X}{Y}\right)^{2}}$ from Equations (4.102) and (4.116). Thus, the squint-mode model reduces to that of broadside when $\theta_{s}=0$, at least for the equations derived thus far. The generalized linear and quadratic phase error equations will be derived next, and it remains to be shown that these equations will (and must) also reduce to the broadside case when the squint is zero.

\subsubsection{Polynomial Phase Terms for Squint-Mode}

As was the case with the broadside collection, a Taylor series expansion will be applied to the point target phase return of Equation (4.44), given the value of $r_{t}$ in Equation (4.42) (which applies to either squint or broadside collections), as well as the new squint-specific relation for $r_{x}$ given by Equation (4.118). Thus, as before, the Taylor series of the phase function $\phi_{x_{0}^{\prime}, y_{0}^{\prime}}\left(X^{\prime}, Y^{\prime}\right)$ is expanded around the aperture 
center point $\left(X_{0}, Y_{0}\right)=\left(0, k_{0}\right)$ such that

$$
\begin{aligned}
\phi_{x_{0}^{\prime}, y_{0}^{\prime}}\left(X^{\prime}, Y^{\prime}\right)= & a_{00}+a_{10} X^{\prime}+a_{01} Y^{\prime}+a_{20} X^{\prime 2} \\
& +a_{11} X^{\prime} Y^{\prime}+a_{02} Y^{\prime 2}+\ldots
\end{aligned}
$$

where

$$
a_{i j}=\left.\left(\frac{1}{i ! j !}\right)\left(\frac{\partial^{i+j} k\left(r_{x}-r_{t}\right)}{\partial X^{i} \partial Y^{j}}\right)\right|_{\substack{X=X_{0}, Y=Y_{0}}}, \quad X_{0}=0, Y_{0}=k_{0}=\frac{4 \pi}{\lambda}
$$

(Equation (4.48))

The constant phase term $a_{00}$ is of no consequence. The terms which are linear in $X^{\prime}$ and $Y^{\prime}$ are image translation terms and specify the linear target displacement in cross-range and range in proportion to $a_{10}$ and $a_{01}$, respectively. In the presence of wavefront curvature, these terms contain extraneous linear components that induce the geometric distortion. Thus $a_{10}$ and $a_{01}$ fully describe the target position in range and cross-range, including the displacement (geometric distortion) due to wavefront curvature. The linear phase coefficient $a_{01}$ for target placement in range for a generalized, squinted collection is defined as follows. From Equation (4.48),

$$
a_{01}=\left.\left(\frac{\partial \phi_{x_{0}^{\prime}, y_{0}^{\prime}}\left(X^{\prime}, Y^{\prime}\right)}{\partial Y}\right)\right|_{\substack{X=0, Y=k_{0}}}
$$

where

$$
\phi_{x_{0}^{\prime}, y_{0}^{\prime}}\left(X^{\prime}, Y^{\prime}\right)=k\left(r_{x}-r_{t}\right)
$$


given

$$
\begin{aligned}
k & =\sqrt{X^{2}+Y^{2}} \\
r_{t} & =\sqrt{r_{x}^{2}+\rho^{2}-2 \rho r_{x} \sin \left(\arctan \left(\frac{X}{Y}\right)+\gamma\right)} \\
r_{x} & =r_{p}\left(1+\Omega_{s}\right) \\
r_{p} & =r_{0} \sqrt{1+\left(\frac{X}{Y}\right)^{2}}, \\
\Omega_{s} & =\frac{\frac{X}{Y} \sin \theta_{s}}{\cos \theta_{s}-\frac{\bar{X}}{Y} \sin \theta_{s}}, \quad-90^{\circ}<\theta_{s}<90^{\circ} \\
x_{0}^{\prime} & =\rho \cos \gamma
\end{aligned}
$$

and

$$
y_{0}^{\prime}=\rho \sin \gamma
$$

Evaluating Equation (4.119) for the generalized, squint-mode range coefficient $a_{01}$ yields the following:

$$
\begin{aligned}
a_{01} & =\left.\left(\frac{\partial \phi_{x_{0}^{\prime}, y_{0}^{\prime}}\left(X^{\prime}, Y^{\prime}\right)}{\partial Y}\right)\right|_{\substack{X=0, Y=k_{0}}} \\
& =r_{0}-r_{c}
\end{aligned}
$$

which is the same result as for the broadside-only case, given by Equation (4.81). Consequently, as previously shown,

$$
\begin{aligned}
r_{c} & =\sqrt{r_{0}^{2}+x_{0}^{\prime 2}+y_{0}^{\prime 2}-2 r_{0} y_{0}^{\prime}} \\
& \approx r_{0}-y_{0}^{\prime}+\frac{x_{0}^{\prime 2}}{2 r_{0}}
\end{aligned}
$$

where $r_{0}$ is the slant range at mid-aperture and $\left(x_{0}^{\prime}, y_{0}^{\prime}\right)$ specifies the point in the imaged slant plane scene for which the phase return is being calculated. Similarly, for the squint-mode case, the linear cross-range phase coefficient $a_{10}$ is calculated 
from Equations (4.48) and (4.120):

$$
\begin{aligned}
a_{10} & =\left.\left(\frac{\partial \phi_{x_{0}^{\prime}, y_{0}^{\prime}}\left(X^{\prime}, Y^{\prime}\right)}{\partial X}\right)\right|_{\substack{X=0, Y=k_{0}}} \\
& =\frac{r_{o}}{r_{c}}\left\{\tan \theta_{s}\left[r_{c}-r_{0}+y_{0}^{\prime}\right]+x_{0}^{\prime}\right\},
\end{aligned}
$$

where $\theta_{s}$ is the squint angle at mid-aperture, at which point the slant range is defined as $r_{0}$. From Equations (4.69) and (4.122), the linear cross-range phase term $\phi_{1 t_{x}}$ is

$$
\begin{aligned}
\phi_{1 t_{x}} & =a_{10} X^{\prime} \\
& =\frac{r_{o}}{r_{c}}\left\{\tan \theta_{s}\left[r_{c}-r_{0}+y_{0}^{\prime}\right]+x_{0}^{\prime}\right\} X^{\prime}, \quad-90^{\circ}<\theta_{s}<90^{\circ} .
\end{aligned}
$$

When the squint angle $\theta_{s}$ is zero, then the broadside scenario applies and this equation reduces to

$$
\left.\phi_{1 t_{x}}\right|_{\theta_{s}=0}=\frac{x_{0}^{\prime} r_{0}}{r_{c}} X^{\prime} \quad \text { (broadside scenario) }
$$

which agrees with (4.70), the broadside model equation. From Equations (4.82) and (4.121), the squint-mode linear phase term in range $\phi_{1 t_{y}}$ is

$$
\begin{aligned}
\phi_{1 t_{y}} & =a_{01} Y^{\prime} \\
& =\left(r_{0}-r_{c}\right) Y^{\prime},
\end{aligned}
$$

which is identical to Equation (4.83) for the broadside model. This is true for all squint angles (not just $\theta_{s}=0$ ), since the equation is independent of the squint angle $\theta_{s}$. By linear combination of Equations (4.123) and (4.125), the total linear phase $\phi_{1 t}$ for the generalized squint-mode scenario is found to be

$$
\begin{aligned}
\phi_{1 t} & =\phi_{1 t_{x}}+\phi_{1 t_{y}} \\
& =a_{10} X^{\prime}+a_{01} Y^{\prime} \\
& =\frac{r_{o}}{r_{c}}\left\{\tan \theta_{s}\left[r_{c}-r_{0}+y_{0}^{\prime}\right]+x_{0}^{\prime}\right\} X^{\prime}+\left(r_{0}-r_{c}\right) Y^{\prime},
\end{aligned}
$$

where $X^{\prime}$ and $Y^{\prime}$ represent the spatial frequency extents in cross-range and range, respectively. This equation describes the total linear phase including the components 
$x_{0}^{\prime} X^{\prime}+y_{0}^{\prime} Y^{\prime}$ that properly place the target in the imaged spatial-domain scene, as well as the extraneous linear distortion terms $f_{\hat{x}^{\prime}} X^{\prime}$ and $f_{\hat{y}^{\prime}} Y^{\prime}$, which serve to misplace the target in cross-range and range, respectively. Hence, the total linear phase could be represented by the form of Equation (4.13), namely

$$
\phi_{1 t}=x_{0}^{\prime} X^{\prime}+y_{0}^{\prime} Y^{\prime}+f_{\hat{x}^{\prime}} X^{\prime}+f_{\hat{y}^{\prime}} Y^{\prime} .
$$

In fact, this was done for the broadside-only linear phase equation of (4.84). As will be discussed in Chapter 5 , it necessary to isolate the distortion terms so that the space-variant post-filter can be correctly applied.

However, in its current form, there appears no straightforward method for the decomposition of Equation (4.126) into its "correct" and distorted terms. This relation is composed of both range and cross-range terms, the combination of Equations (4.123) and (4.125). Since the range term is independent of squint, it can be decomposed as was shown in Equation (4.87) for the broadside-only case. Unfortunately, the cross-range linear phase term of Equation (4.123) is more problematic. There appears no obvious way to isolate the geometric distortion term because of the trigonometric function that is involved. Consequently, the total linear phase $\phi_{1 t}$ of Equation (4.126) can not be decomposed in terms of geometric distortion and "proper placement" components, as will be required in Chapter 5. Furthermore, even for the simpler broadside-only case of Section 4.2, this decomposition required the application of a small patch assumption which was not always appropriate. Fortunately, as will be shown in the next chapter, an elegant mathematical trick can be employed which serves to isolate the geometric distortion component of a given point $\left(x_{0}^{\prime}, y_{0}^{\prime}\right)$ in the presence of wavefront curvature. This is true even when using the exact relation for linear phase; consequently, it applies to all patch sizes and slant ranges with no approximation errors being introduced. Equally fortunate is that the trick is appropriate for the generalized squint-mode linear phase equation and not just the specific broadside relation. Hence, it will be possible to apply space-variant post-filtering to all polar-formatted spotlight-mode imaging scenarios regardless of patch size, squint angle, slant range or resolution. 


\subsubsection{Quadratic Phase Error Term for Squinted Collections}

The Taylor series expansion has been applied to derive the linear components of the phase return from a point target. Earlier, in Section 4.2, this expansion was applied to the broadside-only imaging model, and in this section to the generalized, squint-mode model of which the broadside scenario is a specific case. Next, the quadratic phase error terms for the squint-mode model will be calculated. As was the case for the linear terms, it will be shown that the broadside-mode quadratic terms derived earlier are a special case of the more general squint-mode terms to be derived now. As one would expect, for squinted cases $\left(\theta_{s} \neq 0\right)$ there are additional quadratic phase components which are a function of the squint angle at mid-aperture, $\theta_{s}$. When the squint is zero, the generalized squint-mode quadratic equations reduce to those of the broadside case, as expected. In all cases, quadratic phase serves to defocus the image via IPR broadening, and is space-variant in nature. Perhaps the most surprising (and pleasant) result for the generalized squint-mode scenario is that the defocus remains strictly cross-range only, regardless of the degree of squint. This cross-range-only defocus is observable in squinted imagery where wavefront curvature is significant, yet this is only a qualitative observation. The one-dimensionality of the defocus, even for squinted collections, will now be verified mathematically by showing that the quadratic phase terms $X^{\prime} Y^{\prime}$ and $Y^{\prime 2}$ are indeed zero, regardless of squint angle. Furthermore, in Chapter 6, it will be demonstrated via SYNTARGbased computer simulations that the space-variant defocus is effectively removed for squinted scenarios via the post-filter derived from the new, generalized squint-mode model.

The quadratic terms of the phase return from a point target are calculated from the Taylor series derivation of the generalized, squint-mode phase equation (4.120), where $\phi_{x_{0}^{\prime}, y_{0}^{\prime}}\left(X^{\prime}, Y^{\prime}\right)=k\left(r_{x}-r_{t}\right)$, and are expressed as the sum $\phi_{2 t}$, where

$$
\begin{aligned}
\phi_{2 t} & =f_{\widehat{x^{2}}} X^{\prime 2}+f_{\overparen{x y^{\prime}}} X^{\prime} Y^{\prime}+f_{\widehat{y^{\prime}}} Y^{\prime 2} \\
& =a_{20} X^{\prime 2}+a_{11} X^{\prime} Y^{\prime}+a_{02} Y^{\prime 2} .
\end{aligned}
$$


From Equation (4.48), the specific quadratic coefficients $a_{20}, a_{11}$ and $a_{02}$ are defined as

$$
\begin{aligned}
& a_{20}=\left.\left(\frac{1}{2}\right)\left(\frac{\partial^{2} k\left(r_{x}-r_{t}\right)}{\partial X^{2}}\right)\right|_{\substack{X=X_{X}, Y=Y_{0}}}, \\
& a_{11}=\left.\left(\frac{\partial^{2} k\left(r_{x}-r_{t}\right)}{\partial X \partial Y}\right)\right|_{\substack{X=X_{0}, Y=Y_{0}}},
\end{aligned}
$$

and

$$
a_{02}=\left.\left(\frac{1}{2}\right)\left(\frac{\partial^{2} k\left(r_{x}-r_{t}\right)}{\partial Y^{2}}\right)\right|_{\substack{X=X_{0}, Y=Y_{0}}}
$$

and the coefficients are evaluated at the aperture center, $\left(X_{0}, Y_{0}\right)=\left(0, k_{0}\right)$. First, calculating the quadratic range coefficient $a_{02}$,

$$
\begin{aligned}
a_{02} & =\left.\left(\frac{1}{2}\right)\left(\frac{\partial^{2} k\left(r_{x}-r_{t}\right)}{\partial Y^{2}}\right)\right|_{\substack{X=0, Y=k_{0}}} \\
& =-\frac{1}{2 k_{0}}\left(r_{0}-\sqrt{r_{0}^{2}+\rho^{2}-2 \rho r_{0} \sin \gamma}\right)+\frac{1}{2 k_{0}}\left(r_{0}-\sqrt{r_{0}^{2}+\rho^{2}-2 \rho r_{0} \sin \gamma}\right) \\
& =-\frac{1}{2 k_{0}}\left(r_{0}-r_{c}\right)+\frac{1}{2 k_{0}}\left(r_{0}-r_{c}\right) \\
& =0 .
\end{aligned}
$$

Thus, for the generalized squint-mode scenario, the range oriented quadratic phase error term $f_{\widehat{y^{2}}} Y^{\prime 2}$ is found to be

$$
f_{\bar{y}^{2}} Y^{\prime 2}=a_{02} Y^{\prime 2}=0 Y^{\prime 2}=0
$$

for all squint angles, slant ranges, center frequencies and resolutions. However, it has not yet been shown that defocus due to quadratic phase is strictly one-dimensional in cross-range. This requires the quadratic cross term $f_{\widehat{x y}} X^{\prime} Y^{\prime}$ to be zero, as well. Since $f_{\overparen{x y}} X^{\prime} Y^{\prime}=a_{11} X^{\prime} Y^{\prime}$, the coefficient term $a_{11}$ can be calculated from Equations 
(4.94) and (4.120) as follows:

$$
\begin{aligned}
a_{11}= & \left.\left(\frac{\partial^{2} k\left(r_{x}-r_{t}\right)}{\partial X \partial Y}\right)\right|_{\substack{X=0, Y=k_{0}}} \\
= & \frac{r_{0}}{k_{0}}\left\{\tan \theta_{s}-\frac{1}{r_{c}}\left[r_{0} \tan \theta_{s}-y_{0}^{\prime} \tan \theta_{s}-x_{0}^{\prime}\right]\right. \\
& \left.-\tan \theta_{s}-\frac{1}{r_{c}}\left[-r_{0} \tan \theta_{s}+y_{0}^{\prime} \tan \theta_{s}+x_{0}^{\prime}\right]\right\} \\
= & 0 .
\end{aligned}
$$

Thus, for the generalized squint-mode scenario, the quadratic cross term for phase error, $f_{\widehat{x y^{\prime}}} Y^{\prime 2}$, is found to be

$$
f_{\widehat{x y}} X^{\prime} Y^{\prime}=a_{11} X^{\prime} Y^{\prime}=0 X^{\prime} Y^{\prime 2}=0
$$

for all squint angles, slant ranges, center frequencies and resolutions. Consequently, there are no quadratic phase error components with range orientation, regardless of squint. This is a remarkable result which greatly reduces the computational burden because one-dimensional filtering is sufficient when compensating wavefront curvature defocus effects via a space-variant post-filter.

All that remains is to calculate the quadratic phase term $f_{\widehat{x^{2}}} X^{\prime 2}$ and algebraically combine and rearrange the terms. Since $f_{\widehat{x^{2}}} X^{\prime 2}=a_{20} X^{\prime 2}$ (from Equations (4.91) and (4.92)), the coefficient term $a_{20}$ can be calculated from Equations (4.93) and (4.120) at the aperture center, $\left(X_{0}, Y_{0}\right)=\left(0, k_{0}\right)$ :

$$
\begin{aligned}
a_{20}= & \left.\left(\frac{1}{2}\right)\left(\frac{\partial^{2} k\left(r_{x}-r_{t}\right)}{\partial X^{2}}\right)\right|_{\substack{X=0, Y=k_{0}}} \quad \text { (Equation } \\
= & \frac{1}{2}\left\{\frac{1}{k_{0}}\left(r_{0}-r_{c}\right)+\frac{r_{0}}{k_{0}}\left[1+2 \tan ^{2} \theta_{s}\right]\right. \\
& +\frac{k_{0}}{4 r_{c}^{3}}\left[\frac{2 r_{0}}{k_{0}}\left(r_{0} \tan \theta_{s}-y_{0}^{\prime} \tan \theta_{s}-x_{0}^{\prime}\right)\right]^{2} \\
& \left.-\frac{r_{0}}{r_{c} k_{0}}\left[r_{0}+3 r_{0} \tan ^{2} \theta_{s}-2 y_{0}^{\prime} \tan ^{2} \theta_{s}-2 x_{0}^{\prime} \tan \theta_{s}\right]\right\} .
\end{aligned}
$$


Finally, after some algebraic manipulation to combine and rearrange the terms of $a_{20}$, the quadratic phase term $f_{{\widetilde{x^{2}}}^{\prime}} X^{\prime 2}$ can be expressed by the following relation:

$$
\begin{aligned}
f_{\widehat{x}^{\prime}} X^{\prime 2}= & a_{20} X^{\prime 2} \\
= & \frac{1}{2}\left\{\left[\frac{r_{0}-r_{c}}{k_{0}}-\frac{r_{0}\left(r_{0}-r_{c}\right)}{r_{c} k_{0}}+\frac{x_{0}^{\prime 2} r_{0}^{2}}{r_{c}^{3} k_{0}}\right]\right. \\
& +\frac{2 r_{0} \tan \theta_{s}}{r_{c} k_{0}}\left[x_{0}^{\prime}+\frac{x_{0}^{\prime} y_{0}^{\prime} r_{0}-x_{0}^{\prime} r_{0}^{2}}{r_{c}^{2}}\right] \\
& \left.+\frac{r_{0} \tan ^{2} \theta_{s}}{r_{c} k_{0}}\left[2 r_{c}-3 r_{0}+2 y_{0}^{\prime}+\frac{r_{0}^{3}-2 y_{0}^{\prime} r_{0}^{2}+y_{0}^{\prime 2} r_{0}}{r_{c}^{2}}\right]\right\} X^{\prime 2},
\end{aligned}
$$

where $k_{0}=\frac{4 \pi f_{0}}{c}=\frac{4 \pi}{\lambda}$ is the nominal phase history radius, $r_{0}$ is the slant range to $\mathrm{CRP}$ at mid-aperture, and from Figure 4.5 (a), the distance $r_{c}$ from the platform at mid-aperture to the target at $\left(x_{0}^{\prime}, y_{0}^{\prime}\right)$ is

$$
\begin{aligned}
r_{c} & =\sqrt{r_{0}^{2}+\rho^{2}-2 \rho r_{0} \sin (\gamma)} \\
& =\sqrt{r_{0}^{2}+x_{0}^{\prime 2}+y_{0}^{\prime 2}-2 r_{0} y_{0}^{\prime}} \\
& \approx r_{0}-y_{0}^{\prime}+\frac{x_{0}^{\prime 2}}{2 r_{0}} .
\end{aligned}
$$

Equation (4.132) is a complete, exact specification of the quadratic phase error for the generalized, squint-mode scenario. This closed-form solution, based on the linear flight path model of Figure 4.5, describes the quadratic phase error in Fourier space as a function of the target position $\left(x_{0}^{\prime}, y_{0}^{\prime}\right)$ as imaged in the slant plane. This phase error is one-dimensional in cross-range only and is valid for every resolution, center frequency, slant range, squint angle and patch diameter, where the spotlight-mode aperture is constrained to an angular interval much less than $360^{\circ}$ [2]. Equation (4.132) is arranged such that the first bracketed addend (a) is independent of squint, while the second and third bracketed addends, (b) and (c), respectively, are functions of the squint angle $\theta_{s}$. When $\theta_{s}$ is zero, then the broadside case applies since at midaperture, the platform is looking at a right angle towards the scene center. In this case, $\tan \theta_{s}=\tan (0)=0$, and squint addends (b) and (c) of Equation (4.132) 
disappear. This results in the following broadside-specific relation. From Equation (4.132),

$$
\begin{aligned}
\left.f_{\widehat{x}^{\prime}} X^{\prime 2}\right|_{\theta_{s}=0} & =\left.a_{20} X^{\prime 2}\right|_{\theta_{s}=0} \quad \text { (broadside scenario) } \\
& =\frac{1}{2}\left\{\frac{r_{0}-r_{c}}{k_{0}}-\frac{r_{0}\left(r_{0}-r_{c}\right)}{r_{c} k_{0}}+\frac{x_{0}^{\prime 2} r_{0}^{2}}{r_{c}^{3} k_{0}}\right\} X^{\prime 2} .
\end{aligned}
$$

This is identical to the broadside-only quadratic phase error equation given in (4.98), as would be expected. Thus, as was shown earlier for the geometric distortion terms, the broadside scenario is a specific case of the generalized squint-mode scenario with respect to the quadratic phase error term, as well.

It is useful to present a simplified approximation of the quadratic phase error of Equation (4.132), based on the small patch assumption where $x_{0}^{\prime}, y_{0}^{\prime} \ll r_{0}$. Although this simplified relation will allow faster phase error calculations (in terms of CPU multiplies) given the specific scenarios for which the approximation holds, this is not the main benefit of a simpler mathematical form. Instead, it helps us to better visualize and understand the precise effect (detriment) of squint-mode scenarios on quadratic phase error, compared to imaging the same scene without squint. When the small patch assumption is applied, some additional mathematical manipulation yields the following relation for squint-mode quadratic phase error:

$$
\begin{aligned}
f_{{\widehat{x^{2}}}^{\prime}} X^{\prime 2} & =\phi_{2 t} \\
& \approx \frac{1}{2}\left\{\frac{x_{0}^{\prime 2}-y_{0}^{\prime 2}}{r_{0} k_{0}}-\tan \theta_{s}\left[\frac{2 x_{0}^{\prime} y_{0}^{\prime}}{r_{0} k_{0}}\right]-\tan ^{2} \theta_{s}\left[\frac{x_{0}^{\prime 2} y_{0}^{\prime}}{\dot{r}_{0}^{2} k_{0}}\right]\right\} X^{\prime 2}, \quad x_{0}^{\prime}, y_{0}^{\prime} \ll r_{0} .
\end{aligned}
$$

As before, the equation is separated into addends which represent their reliance on squint. The first addend is independent of squint while the second and third are functions of the squint angle $\theta_{s}$, as shown. When squint is zero, only the first term remains, as shown.

$$
\begin{aligned}
\left.f_{\bar{x}^{\prime}} X^{\prime 2}\right|_{\theta_{s}=0} & =\left.\phi_{2 t}\right|_{\theta_{s}=0} \quad \text { (broadside scenario approximated) } \\
& \approx \frac{1}{2}\left\{\frac{x_{0}^{\prime 2}-y_{0}^{\prime 2}}{r_{0} k_{0}}\right\} X^{\prime 2}, \quad x_{0}^{\prime}, y_{0}^{\prime} \ll r_{0} .
\end{aligned}
$$


In this broadside-specific case, this equation reduces such that it is identical to that of the small-patch approximated, broadside-only scenario described by Equation (4.100).

The quadratic phase error approximation given by Equation (4.134) is simple and revealing. It helps to aid in the understanding of how squint affects the overall quadratic phase; specifically, how the new squint terms contribute (additively) to the overall phase error, relative to the broadside phase term. When considering the contribution of just the two squint addends, note that the first (with the $\tan \theta_{s}$ coefficient) contains an $x_{0}^{\prime} / r_{0}$ term. The second squint addend (with the $\tan ^{2} \theta_{s}$ coefficient), an $x_{0}^{\prime 2} / r_{0}^{2}$ term. The first squint addend dominates the second because when $x_{0}^{\prime} \ll r_{0}$, then $x_{0}^{\prime 2} / r_{0}^{2} \ll x_{0}^{\prime} / r_{0}$. Consequently, the second can be ignored when considering the contribution of squint to the overall phase error. The effect of the first squint addend is to double the maximum quadratic phase error in a scene covering a square (or circular) ground patch, over the same patch imaged at broadside. However, by no means does this double computation time, as will be discussed in Chapter 6.

As one might suspect (but has not yet been shown), a higher quadratic phase error is more costly in terms of computation time when post-filtering. The additional squint addends do increase the overall quadratic phase error and consequently, the computation time. However, as will be shown in Chapter 6, Section 6.3, even for moderately severe squint angles $\left(-45^{\circ} \leq \theta_{s} \leq 45^{\circ}\right)$, the overall post-filtering computational burden is increased by as little as $5.5 \%$ over that of the same scene imaged at broadside. This is in stark contrast to some other image formation algorithms such as RMA, whose oversampling requirement in squinted scenarios drastically increases computation time [12, pp. 401-438]. 


\subsection{Summary of Phase Error Analysis}

Spotlight-mode image formation via the polar-formatting algorithm has traditionally assumed that planar wavefronts illuminate the imaged scene. This assumption is faulty in certain imaging scenarios; specifically, when the scene is large, the radar center frequency low, the resolution high or the slant range short. In these scenarios, the actual spherical wavefronts differ sufficiently from the planar assumption such that linear, quadratic, and higher-order residual phase terms appear. The linear terms lead to a geometric distortion of the imaged scene, while the higher-order terms lead to a space-variant defocus of the scene.

The spotlight-mode SAR formulation, first introduced by Walker in [2], describes these phase errors in terms of a Taylor series expansion and discusses the limitations of the spotlight-mode approach with respect to these phase errors. Walker also mentions the possibility of compensating the phase errors arising from wavefront curvature, yet deems the problem "difficult to implement" because the errors depend on both aperture position and target position in the scene, rendering the problem computationally inefficient. Later, Munson [4] and Ausherman [5] cast spotlightmode image formation as a tomographic process and describe the planar wavefront assumption as a shortcoming of this tomographic paradigm. Specifically, when the planar wavefront assumption is violated, there no longer exists a direct Fourier relationship between the phase history data and the reconstructed image. Instead, extraneous phase terms are present and lead to the geometric distortion and spacevariant defocus.

In this dissertation, a geometric model was presented in Sections 4.1 and 4.2 that represents the phase return from a point target for a linear flight trajectory, from a tomographic perspective. This model was first proposed by Jakowatz in [7, pp. 355-365] and applies to broadside (non-squint) spotlight-mode collections. Jakowatz makes no assumptions regarding the spherical nature of the wavefronts, and a mathematical analysis of the phase return from a point target yields an expression 
for the linear and quadratic phase errors in closed-form (eg: for a linear flight path), via a small patch approximation. These phase errors arise from the faulty assumption of planar wavefronts illuminating the scene. Jakowatz then demonstrates in [10] that a space-variant post-filter, based on the closed-form phase error equations, adequately compensates the defocus for the specific case of a small patch, broadside collection. In that paper, polar-formatting with space-variant post-filtering was demonstrated to be competitive with other image formation methods, all of which inherently compensate wavefront curvature. However, computational parameters for the space-variant post-filter were picked ad hoc and no emphasis was placed on optimizing computational efficiency. Furthermore, no model had yet been developed to deal with squint-mode collections.

The unique contribution of this dissertation chapter, detailed in Section 4.4 and briefly introduced by Doren in [11], is a closed-form description of the linear and quadratic phase errors that apply not only to broadside collections, but also the more general squinted collection scenarios. It was shown here that the original broadsidemode equations of Jakowatz are a special case of the general squint-mode equations. Furthermore, these generalized relations describe the linear and quadratic phase errors for all patch sizes, resolutions, slant ranges and resolutions, without requiring a small patch assumption, as did Jakowatz. Thus, they are applicable to many practical imaging scenarios, without unduly restricting the collections to broadsideonly or introducing approximation errors into the phase equations. These equations are based on a new, generalized point target phase return model based on that of Jakowatz, in conjunction with a Taylor series expansion of the phase return equation, as proposed by Walker in [2] for broadside scenarios.

It has now been shown that the quadratic phase term induces a one-dimensional defocus in cross-range only, regardless of squint angle, for the linear trajectory being modeled. In spite of Walker's predictions, Chapters 5 and 6 will demonstrate how this equation can be applied to a space-variant post-filter which efficiently and effectively compensates wavefront curvature in squinted spotlight-mode imagery formed 
via polar-formatting. At this point, in spite of Walker's claims, it has already been demonstrated via the linear trajectory model of Figure 4.5 that there is no need to calculate the phase error at individual aperture points. That is, the model yields a closed-form solution that is independent of aperture position. Furthermore, Walker failed to realize that the defocus is one dimensional in cross-range, even for squinted scenarios. In the following chapters, it will be shown that wavefront curvature compensation need not be calculated for every individual point in the imaged scene, as Walker prescribes. Instead, the space-variant post-filter is varied only as often as necessary to maintain a minimal residual phase error, which results in imperceptible image defocus. Consequently, the space-variant post-filter will be shown to be computationally efficient, such that PFA will remain competitive with other image formation algorithms even when compensating the defocus effects of wavefront curvature.

In summary, the concepts of this chapter and the results of Equations (4.47), (4.126) and (4:132) lead to the following equation describing the phase return from a point target, without the approximations associated with planar wave or smallpatch assumptions. It is general in that it applies to all squint angles, slant ranges, resolutions, frequencies and patch sizes.

$$
\begin{aligned}
\phi_{x_{0}^{\prime}, y_{0}^{\prime}}\left(X^{\prime}, Y^{\prime}\right)= & a_{00}+a_{10} X^{\prime}+a_{01} Y^{\prime}+a_{20} X^{\prime 2} \\
& +a_{11} X^{\prime} Y^{\prime}+a_{02} Y^{\prime 2}+\ldots \\
= & \frac{r_{o}}{r_{c}}\left\{\tan \theta_{s}\left[r_{c}-r_{0}+y_{0}^{\prime}\right]+x_{0}^{\prime}\right\} X^{\prime}+\left(r_{0}-r_{c}\right) Y^{\prime} \\
& +\frac{1}{2}\left\{\left[\frac{r_{0}-r_{c}}{k_{0}}-\frac{r_{0}\left(r_{0}-r_{c}\right)}{r_{c} k_{0}}+\frac{x_{0}^{\prime 2} r_{0}^{2}}{r_{c}^{3} k_{0}}\right]\right. \\
& +\frac{2 r_{0} \tan \theta_{s}}{r_{c} k_{0}}\left[x_{0}^{\prime}+\frac{x_{0}^{\prime} y_{0}^{\prime} r_{0}-x_{0}^{\prime} r_{0}^{2}}{r_{c}^{2}}\right] \\
& \left.+\frac{r_{0} \tan ^{2} \theta_{s}}{r_{c} k_{0}}\left[2 r_{c}-3 r_{0}+2 y_{0}^{\prime}+\frac{r_{0}^{3}-2 y_{0}^{\prime} r_{0}^{2}+y_{0}^{\prime 2} r_{0}}{r_{c}^{2}}\right]\right\} X^{\prime 2} \\
& +\ldots .
\end{aligned}
$$

This equation is a Taylor series representation of the total Fourier domain phase as 
a function of spatial position the image domain, and includes the linear distortion terms and quadratic cross-range defocus term that arise from the planar wavefront assumption when used in the presence of wavefront curvature. While higher-order error terms exist, they are typically inconsequential, as discussed in Chapter 6 . 


\section{Chapter 5}

\section{Space-Variant Post-Filter Design}

In the previous chapter, a closed-form, analytic expression was developed to describe the quadratic phase error in the presence of wavefront curvature. This phase error, due to the faulty planar wavefront assumption in the tomographic paradigm, renders invalid the direct Fourier relationship between the polar-formatted phase history and the scene being illuminated. Without proper quadratic phase compensation, a spacevariant, cross-range defocus is induced in the formed imagery, and for some imaging scenarios, is severe enough to significantly reduce resolution. This degradation is particularly severe along the Cartesian axes at the far edges of large scenes, when imaging at close range, using low center frequencies or at high spatial resolutions. The expression previously derived for quadratic phase (Equation (4.132)) accurately describes this error for all scene sizes, slant ranges, center frequencies, resolutions and squint angles, for a specified linear platform trajectory. Furthermore, it was shown that the defocus remains one-dimensional in cross-range even for squinted scenarios, which greatly reduces computational burden. Also, it was suggested that the overall computational burden is reasonable because phase corrections need not be applied at every aperture point, and that the additional burden associated with squinted scenes is typically less than $6.9 \%$ (as will be verified in Chapter 6).

Exactly how is the quadratic phase error compensated? That is, based on the 
space-variant quadratic phase error equation presented previously, by what process is the polar-formatted spotlight-mode image refocused when degraded by wavefront curvature defocus effects? That is the topic of this chapter. The space-variant defocus effects are compensated in the image domain by what is considered an image domain deconvolution process. The beauty of this process is that it is performed as a separate post-processing step to PFA, and therefore, is independent of the image-formation process. Only basic radar parameters and simple imaging geometry descriptions are required to satisfy the analytic quadratic phase error equation, and consequently, image refocus is accomplished based strictly on these parameters and the spatial position in the scene, without dependence on the original collected Fourier phase history or knowledge of scene content. This deconvolution process, also known as a restoration filter or inverse filter, and referred to here as a space-variant postfilter, frees us from the severe scene size restrictions imposed by polar-formatting in the presence of wavefront curvature, as discussed in Chapter 3. Furthermore, spacevariant post-filtering is straightforward to implement and computationally efficient, as it adds as little as an additional $25 \%$ to the polar-format computation time.

\subsection{The Concept of Wavefront Curvature Correc- tion Via Space-Variant Post-Filtering}

The one-dimensional, cross-range defocus (IPR broadening) in spotlight-mode imagery processed via polar-formatting will be compensated via a space-variant postfilter, whose parameters are specified by the quadratic phase error equations of Chapter 3. While this process may be thought of as an image domain deconvolution process, it may equivalently be considered a Fourier-domain phase correction process. Since the phase return models and subsequent quadratic phase error equations derived in Chapter 3 are in terms of Fourier space, it is convenient to discuss the space-variant post-filter in terms of a frequency domain phase correction process. 
Furthermore, except for the smallest of spatial domain convolution kernels, this inverse filtering process is often more computationally efficient in the Fourier domain. The filter, as applied to a location in Fourier space, is a quadratic phase function derived from the phase error equation at that particular $\left(x_{0}^{\prime}, y_{0}^{\prime}\right)$ Cartesian location in the spatial domain. Since this equation specifies the Fourier phase error contribution at that given point, a complex-conjugate multiply of the filter values with the complex-valued Fourier data results in the appropriate compensation (negation) of the phase error within the Fourier data. Alternatively, in the spatial domain, wavefront curvature correction consists of a deconvolution process whereby the convolution kernel is the inverse Fourier transform of the quadratic phase error function for that point in the scene.

The corrective post-filter values are based on the quadratic phase error equation (or its approximation, as appropriate), as derived in Chapter 3. This equation is repeated here for convenience. The Fourier domain quadratic phase error due to wavefront curvature $\phi_{2 t\left(x_{0}^{\prime}, y_{0}^{\prime}\right)}\left(X^{\prime}, Y^{\prime}\right)$, which is a function of spatial slant range position $\left(x_{0}^{\prime}, y_{0}^{\prime}\right)$, was given in Equation (4.132) and found to be

$$
\begin{aligned}
\phi_{2 t\left(x_{0}^{\prime}, y_{0}^{\prime}\right)}\left(X^{\prime}, Y^{\prime}\right)= & \phi_{2 t\left(x_{0}^{\prime}, y_{0}^{\prime}\right)}\left(X^{\prime}\right) \\
= & \frac{1}{2}\left\{\left[\frac{r_{0}-r_{c}}{k_{0}}-\frac{r_{0}\left(r_{0}-r_{c}\right)}{r_{c} k_{0}}+\frac{x_{0}^{\prime 2} r_{0}^{2}}{r_{c}^{3} k_{0}}\right]\right. \\
& +\frac{2 r_{0} \tan \theta_{s}}{r_{c} k_{0}}\left[x_{0}^{\prime}+\frac{x_{0}^{\prime} y_{0}^{\prime} r_{0}-x_{0}^{\prime} r_{0}^{2}}{r_{c}^{2}}\right] \\
& \left.+\frac{r_{0} \tan ^{2} \theta_{s}}{r_{c} k_{0}}\left[2 r_{c}-3 r_{0}+2 y_{0}^{\prime}+\frac{r_{0}^{3}-2 y_{0}^{\prime} r_{0}^{2}+y_{0}^{\prime 2} r_{0}}{r_{c}^{2}}\right]\right\} X^{\prime 2},
\end{aligned}
$$

where $k_{0}=\frac{4 \pi f_{0}}{c}=\frac{4 \pi}{\lambda}$ is the nominal phase history radius, $r_{0}$ is the slant range from platform to CRP at mid-aperture, $r_{c}$ is the distance from the platform at midaperture to the target positioned at $\left(x_{0}^{\prime}, y_{0}^{\prime}\right)$, and $\theta_{s}$ is the squint angle (measured from broadside). Furthermore, $X^{\prime}$ is the cross-range frequency extent. The half- 
power width of the IPR, $X_{1}^{\prime}$, defines the maximum frequency extent of the aperture such that $\left(\left|X^{\prime}\right| \leq X_{1}^{\prime}\right)$, where

$$
X^{\prime}=\frac{\pi}{\rho_{x}}
$$

and $\rho_{x}$ is the image resolution in cross-range. Thus, it is obvious that the quadratic phase error of Equation (5.1) increases as the square of the resolution in cross-range.

When the patch being imaged is small compared to the slant range; that is, when $x_{0}^{\prime}, y_{0}^{\prime} \ll r_{0}$, then the small patch assumption applies, and Equation (5.1) can be approximated by

$$
\begin{array}{r}
\phi_{x_{0}^{\prime}, y_{0}^{\prime}}\left(X^{\prime}\right) \approx \frac{1}{2}\left\{\frac{x_{0}^{\prime 2}-y_{0}^{\prime 2}}{r_{0} k_{0}}-\tan \theta_{s}\left[\frac{2 x_{0}^{\prime} y_{0}^{\prime}}{r_{0} k_{0}}\right]-\tan ^{2} \theta_{s}\left[\frac{x_{0}^{\prime 2} y_{0}^{\prime}}{r_{0}^{2} k_{0}}\right]\right\} X^{\prime 2}, \\
x_{0}^{\prime}, y_{0}^{\prime} \ll r_{0} .
\end{array}
$$

When the squint angle $\theta_{s}$ is zero, then the broadside-specific case applies and the approximation of Equation (5.4) reduces to

$$
\left.\phi_{x_{0}^{\prime}, y_{0}^{\prime}}\left(X^{\prime}\right)\right|_{\theta_{s}=0} \approx \frac{1}{2}\left\{\frac{x_{0}^{\prime 2}-y_{0}^{\prime 2}}{r_{0} k_{0}}\right\} X^{\prime 2}, \quad x_{0}^{\prime}, y_{0}^{\prime} \ll r_{0}
$$

Equations (5.4) and (5.5) represent Equations (4.134) and (4.135), respectively, and are repeated here for convenience. The design of the space-variant post-filter, as presented in the remainder of this chapter, is based on these equations, as is much of the computational analysis of Chapter 6. Specifically, filter length (patch size), to be discussed next, is derived from Equation (5.5). While this equation is an appropriate quadratic phase error approximation only when small patches are considered, it will serve as the basis for all filter patch size estimates, regardless of patch size of slant range. That is, Equation (5.5), while not an exact expression, is sufficiently accurate to serve as the basis for these calculations. 


\subsection{Space Invariance - Scope of Application}

The quadratic phase relation of Equation (5.1) produces different values as a function of spatial position in the image domain. As such, it is space-variant, dependent on image location in both range and cross-range. By applying an appropriate spatiallyvarying filter to the image that is formed by the polar-format processor, the defocus effects induced by Equation (5.1) are compensated. In theory, Equation (5.1) specifies the filter to be applied to each Cartesian coordinate in the polar-formatted Fourier space. If the filter were changed at every pixel in strict accordance with the expression of (5.1), the computational burden associated with the filter implementation could become excessive. Thus, as was pointed out by Walker in [2], this procedure is computationally inefficient. However, the key to computational efficiency, as proposed in this dissertation, is to vary the post-filter only as often as necessary to maintain a tolerable (ie: imperceptible) amount of residual blur. That is, by applying the space-variant post-filter to patches of imagery, as opposed varying it for each individual $\left(x_{0}^{\prime}, y_{0}^{\prime}\right)$ location, the computational burden is significantly decreased while defocus is. constrained to an acceptable level. Specifically, an image patch size is calculated such that the residual quadratic phase error at the edges, after correction, is within subpixel levels.

Since the filter is applied to complex-valued spatial imagery, yet is specified in terms of the Fourier domain quadratic phase error, the specified image patch is first Fourier transformed, then conjugate multiplied by the filter coefficients, as calculated at the patch center $\left(x_{0}^{\prime}, y_{0}^{\prime}\right)$. This patch is then inverse Fourier transformed into a refocused portion of the formed image. In order to avoid visually perceptible discontinuities at the junctions of imaged patches, the refocus filter must overlap the previous to some degree. In other words, there is overlap in the image patches. The amount of filter overlap, and patch size in general, is the focus (no pun intended) of the remainder of this chapter. Assuming the patch size is fairly large, and the overlap reasonably small, then refocus is accomplished with an acceptable computational 
burden. As will be shown, this is indeed the case for space-variant post-filtering for wavefront curvature correction.

The idea of a closed-form phase correction model for compensating quadratic phase errors is not new and not necessarily limited to wavefront curvature issues. However, only for wavefront curvature, as demonstrated in this dissertation (and also the preliminary work of Jakowatz [7, pp. 355-365][10] and Doren [11] on which it is based), has this method been shown to be computationally efficient. For example, in a paper by $\mathrm{K}$. Kong [86], a quadratic phase correction is applied to compensate the defocus that results when the polar-formatting step of spotlight-mode image formation is omitted. It is hoped that the space-variant polar-format blur is more efficiently compensated via post-filtering, as opposed to the costly two-dimensional sinc interpolation required for polar-formatting. However, as Kong points out, the large amount of polar-format defocus present, in addition to its two-dimensional nature, renders post-filtering to be very inefficient for this type of phase error, except for the smallest of angular extents subtended by the radar platform. However, as will be demonstrated in Chapter 6 of this dissertation, the one-dimensional nature of wavefront curvature defocus, in conjunction with a space-variant post-filter that is varied only as often as necessary, yields an effective method for the removal of wavefront curvature defocus from polar-formatted imagery. Consequently, while Kong demonstrated the mathematical validity of space-variant post-filtering for polar-format defocus, the large magnitude of those errors offset the potential computational advantage of post-filtering. In the remainder of this dissertation, the mathematical theory given for wavefront curvature correction will be (partially) validated via SYNTARG examples. Furthermore, since the wavefront curvature errors are typically much smaller in magnitude than those of polar-format defocus, the efficiency of post-filtering is shown for wavefront curvature correction, whereas Kong failed to demonstrate this for polar-format defocus.

Consider an experiment in which ideal point targets (IPRs) are synthetically generated according to the L-Band parameters in Table 5.1. It has been suggested 


\begin{tabular}{||l|c|c||}
\hline \multicolumn{3}{|c|}{ L-Band Parameters for SYNTARG Simulation } \\
\hline \multicolumn{1}{|c|}{ Parameter } & Symbol & Value \\
\hline \hline Center Frequency & $f_{0}$ & $1.25 \mathrm{gHz}$ \\
Wavelength & $\lambda,\left(\lambda=c / f_{0}\right)$ & $0.24 \mathrm{~m}$ \\
Aperture Extent & $\Delta \theta$ & $10^{\circ}$ \\
Scale Factor, Cross-range & $s_{x}$ & $\approx 0.65 \mathrm{~m} / \mathrm{pix}$ \\
Scale Factor, Range & $s_{y}$ & $\approx 0.65 \mathrm{~m} / \mathrm{pix}$ \\
Mainlobe Broadening Factor & $K_{a}$ & 1.4 \\
Resolution, Cross-range & $\rho_{x}$ & $\approx 1.25 \mathrm{~m}$ \\
Resolution, Range & $\rho_{y}$ & $\approx 1.25 \mathrm{~m}$ \\
Oversample Value, Cross-range & $o_{x}\left(o_{x}=\rho_{x} / s_{x}\right)$ & $\approx 1.92$ \\
Oversample Value, Range & $o_{y}\left(o_{y}=\rho_{y} / s_{y}\right)$ & $\approx 1.92$ \\
Slant Range at Broadside & $r_{0}$ & $4972 \mathrm{~m}$ \\
Squint Angle & $\theta_{s}$ & $0^{\circ}$ \\
Patch Diameter & $D_{p}$ & $2400 \mathrm{~m}$ \\
\hline
\end{tabular}

Table 5.1: L-Band Parameters for Broadside, Space-Invariant Experiment

in [12, pp. 435-439] [87, 88] that L-Band. SARs, under these imaging scenarios, exhibit the defocus effects associated with wavefront curvature. This is also true of Ultra-Wideband (UWB) SARs [12, pp. 437-438][83, 89], whose center frequency, at approximately $500 \mathrm{mHz}$, is approximately one third that of the L-Band frequency. Since the resolution in cross-range $\rho_{x}$ is defined by $\rho_{x}=\lambda /(2 \Delta \theta)$, the UWB SAR is able to attain cross-range resolutions equal to that of the L-Band SAR by subtending an aperture angle $\Delta \theta$ that is three times that which is needed for the.L-Band radar. Furthermore, the lower UWB frequency can potentially induce much greater defocus due to wavefront curvature. However, the limited patch sizes of the UWB SAR, as compared to L-Band, help constrain this defocus [12, p. 438]. Since the separable, two-dimensional sinc interpolator used for polar reformatting may induce its own phase errors with the large angular diversities associated with UWB SARs [71, 72], this dissertation concentrates strictly on L-Band examples so that any phase errors present are of known origin; specifically, from wavefront curvature.

As was described in Chapter 4, a virtue of synthetic targets formed via SYNTARG (as is the case with this example) is that SYNTARG does not assume planar 
Chapter 5. Space-Variant Post-Filter Design

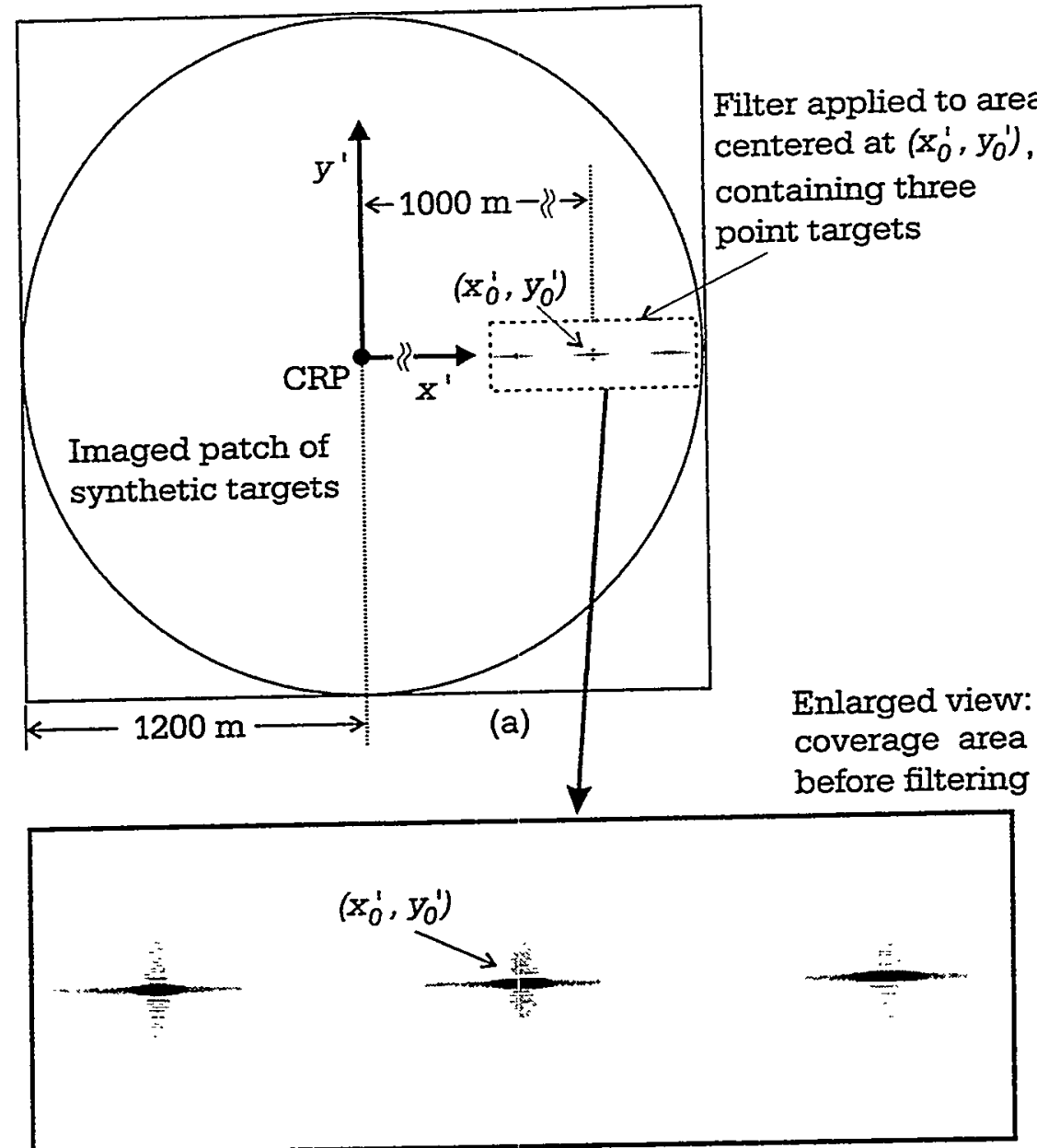

(b)

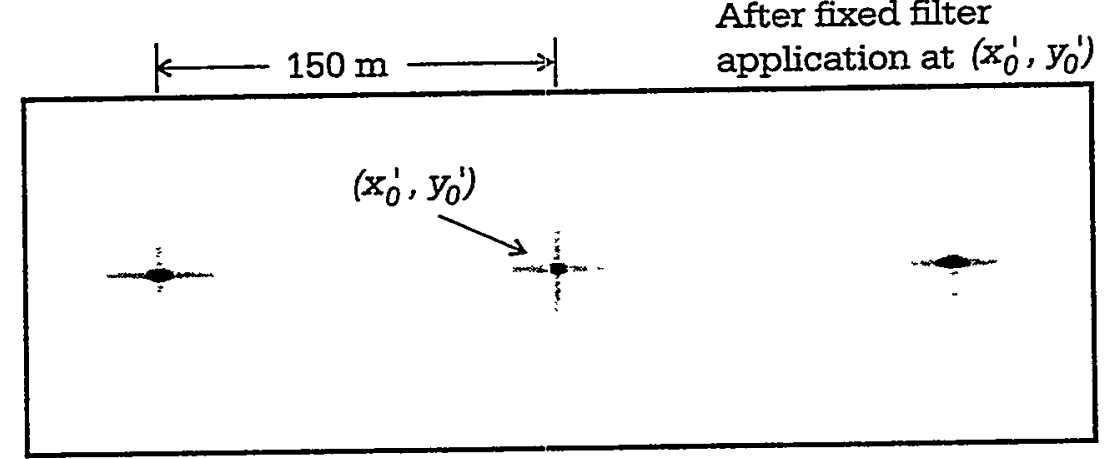

(c)

Figure 5.1: Wavefront Curvature Correction of Synthetic Point Targets via a SpaceInvariant Post-Filter Centered at $\left(x_{0}^{\prime}, y_{0}^{\prime}\right)$ 
wavefronts illuminating the imaged scene [7, pp. 399-414]. Consequently, any perceptible amount of defocus due to wavefront curvature will be evident in the point targets being generated. The example of Figure 5.1 illustrates synthetically generated L-Band targets whose parameters are specified in Table 5.1. In part (a) of this figure, three synthetic targets are shown at far cross-range, along the $x^{t}$ axis in the slant plane. The center target is at $\left(x_{0}^{\prime}, y_{0}^{\prime}\right)=(1000,0)$, which according to Equation (5.5), is a location of severe wavefront curvature defocus. As is clearly seen, these targets exhibit defocus (IPR broadening) in cross-range and the blur is more severe for targets nearer the edge of the scene, as shown in the expanded view of part (b). A space-invariant post-filter has been applied to this region of the image and the result is illustrated in Figure 5.1 (c). The filter parameters were based on the quadratic phase error equation (5.1). In addition, a 40-dB Taylor window was applied for sidelobe reduction. For this example, the patch center was chosen at $\left(x_{0}^{\prime}, y_{0}^{\prime}\right)=(1000,0)$, and the quadratic phase error correction calculated from this single chosen coordinate was applied to the entire target region, as depicted by the dotted lines in Figure 5.1 (a). This region covers $390 \mathrm{~m}$ in cross-range, or 600 pixels given a scale factor of $0.65 \mathrm{~m} /$ pixel. As can be seen in part (c), the center point target residing at $\left(x_{0}^{\prime}, y_{0}^{\prime}\right)=(1000,0)$, is refocused quite well. Those targets to the left and right of the patch center show improved focus, but not to the same degree as the center target. Thus, the need for space-variant post-filtering has been demonstrated. While the quadratic phase correction calculated at some spatial position $\left(x_{0}^{\prime}, y_{0}^{\prime}\right)$ precisely compensates the defocus at that point, it serves only as an approximation to the quadratic phase error induced by wavefront curvature for points surrounding $\left(x_{0}^{\prime}, y_{0}^{\prime}\right)$. Just how large a patch can be refocused for a given patch center, while still maintaining an acceptably small residual blur, is the topic of rest of this chapter.

The space-invariant filter of this example is applied to a small patch of the imagedomain scene of Figure 5.1 (a). However, as previously stated, this portion of the image is first Fourier transformed, then conjugate multiplied by the corrective quadratic phase function before being inverse transformed into a focused patch. The effect of 
Fourier transforming a small patch of the image domain is a resultant Fourier domain phase history that supports the full frequency extent of the image, yet at a less dense (decimated) sampling compared to the original phase history [7, pp. 140144]. Consequently, the phase correction applied in the Fourier domain is a quadratic function of $X^{\prime 2}$ (and $\left(x_{0}^{\prime}, y_{0}^{\prime}\right)$ ) as given by Equations (5.1-5.5), where $X^{\prime}$ is a discrete, decimated representation of the Fourier frequency extent. Specifically, for an $n$ pixel patch centered at $\left(x_{0}^{\prime}, y_{0}^{\prime}\right)$, the sampled frequency extent in the Fourier domain is defined as follows:

$$
X^{\prime}=\sum_{i=0}^{n-1}-X_{1}^{\prime}+\frac{2 i X_{1}^{\prime}}{n-1}, \quad\left|X^{\prime}\right| \leq X_{1}^{\prime} .
$$

The resulting sampled frequency extent consists of $n$ pixels in the frequency range $\left[-X_{1}^{\prime} . . X_{1}^{\prime}\right]$, where $X_{1}^{\prime}$ is the maximum of the absolute value of $X^{\prime}$, and $X^{\prime}$ is calculated from Equation (5.3). The frequency spacing $\delta X^{\prime}$ between samples is then

$$
\delta X^{\prime}=\frac{2 X_{1}^{\prime}}{n-1}, \quad\left[-X_{1}^{\prime} . . X_{1}^{\prime}\right]
$$

Figure 5.2 presents an enlarged view of the space-invariant, post-filtered targets of the previous figure. This enlarged view more clearly illustrates the refocus of the center target and the less clearly focused adjacent targets. In part (b), the results are shown for the original defocused targets, except in this case, a spacevariant filter has been incorporated. Specifically, patches 128 pixels in cross-range are refocused, each with the appropriate $\left(x_{0}^{\prime}, y_{0}^{\prime}\right)$ center coordinate used for the quadratic phase error calculation. Each filter operation was displaced in cross-range by sixty four pixels from that of the previous. That is, the filter had a length $m$ of 128 pixels and the space-variant coefficients were recalculated and the filter applied every 64 pixels. This 64 pixel displacement, $d$, infers a filter overlap region $c$ of 128 $64=64$ pixels. For now, it will suffice to say that this particular filter length, overlap and displacement is appropriate for the proper space-variant refocus of this L-Band example. The remaining sections of this chapter describe in detail how these parameters are to be chosen, both for this example and for all imaging scenarios 


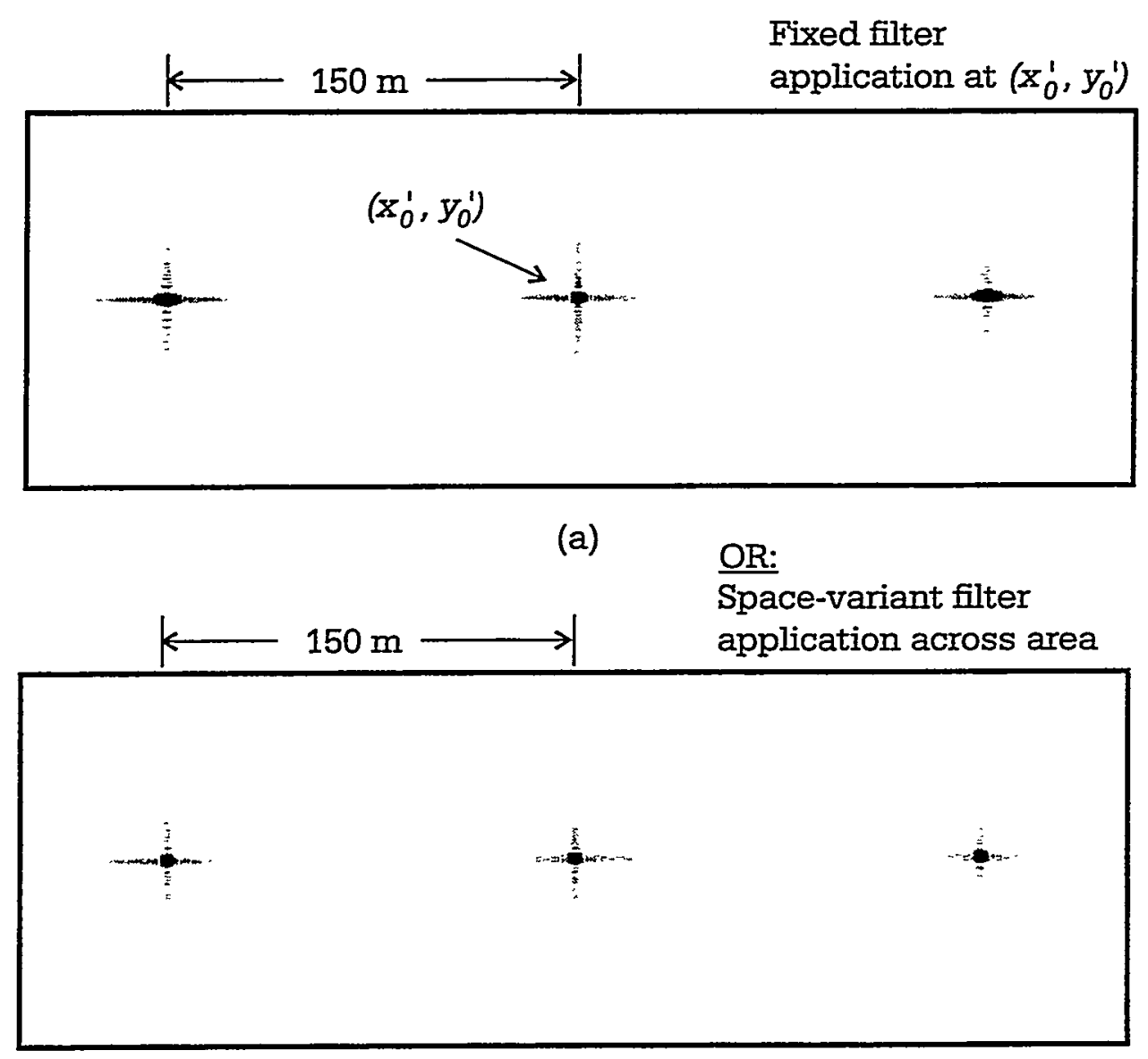

(b)

Figure 5.2: Space-Invariant Vs. Space-Variant Filtering

in general. As can be seen in 5.2 (b), the filter appears to do an excellent job of refocusing all the point targets. Just how well, in a quantitative sense, these targets have been refocused and the computational cost incurred, are the subjects of Chapter 6. While not explicitly shown, the patch is actually two-dimensional in shape. However, because the quadratic defocus is one-dimensional in nature, only one-dimensional Fourier transforms, oriented in cross-range, are necessary for the refocus. Furthermore, no overlap is required in range since there is no quadratic phase component in this direction. Consequently, this filtering operation is much less computationally burdensome than would be the case for two-dimensional filtering. 


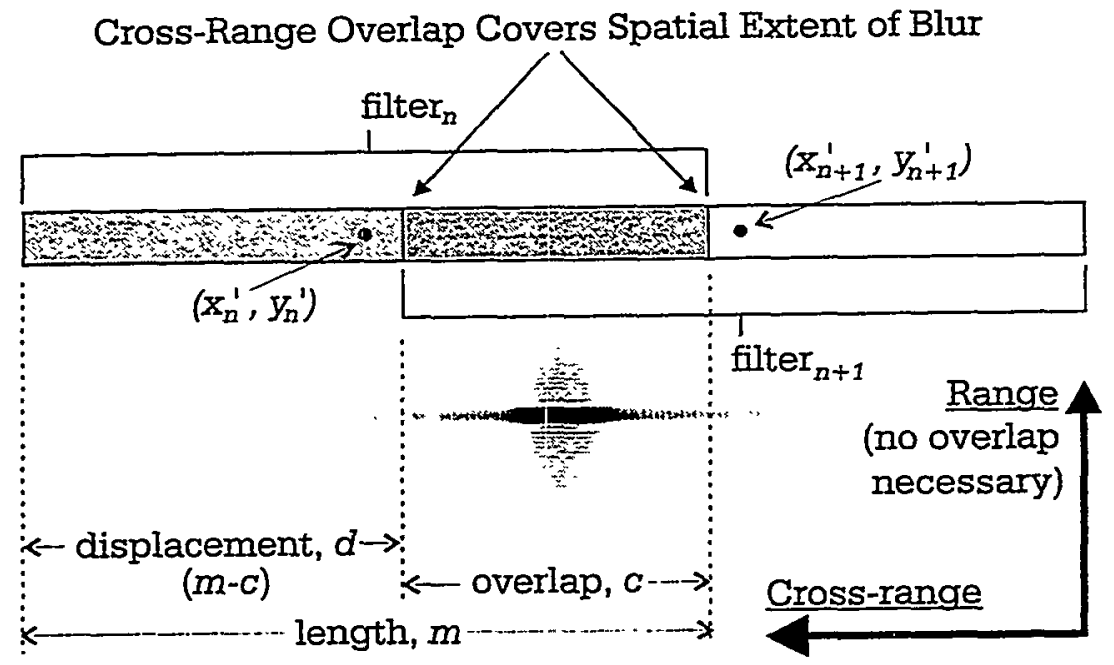

Figure 5.3: Filter Overlap, Displacement and Length

The illustration of Figure 5.3 shows the relationship between filter length $m$, displacement $d$ and overlap $c$, for two adjacent filter operations. This concept is extended to the two-dimensional example depicted in Figure 5.4, showing the subdivision of an image into two patches, which are independently refocused and then seamed back together. It is important to realize that this particular illustration is completely fabricated and consists of neither real imagery nor even synthetic (mathematically valid) point target data. It is the only illustration not representative of the synthetic data based on the mathematical model for the phase return from a point target. However, it is helpful in clepicting the process of space-variant post filtering in two dimensions. In particular, note that the overlap between patches is sufficiently wide to capture the worst-case extent of the blur as seen in the image domain. Furthermore, when two patches displaced by $d$ pixels are seamed back together, only the innermost $d$ pixels for that patch (as centered around $\left(x_{0}^{\prime}, y_{0}^{\prime}\right)$ ) are retained. This process ensures that regardless of the point target position or spatial extent of the blur, the target is not "missed" or partially encompassed in the process of applying the space-variant post-filter to the patches. 

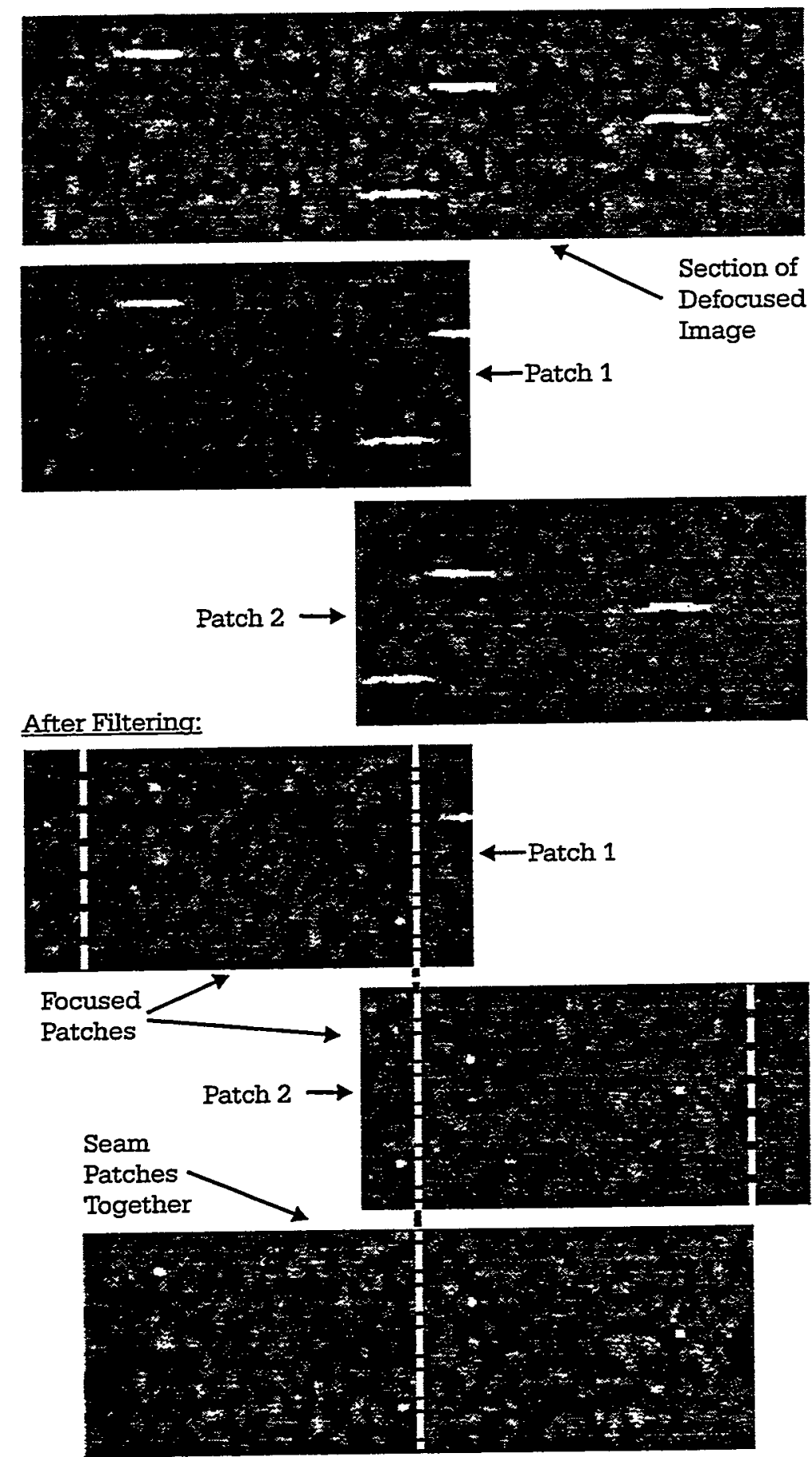

Figure 5.4: Implementation of Space-Variant Post-Filter for Two Patches 


\subsection{Accounting For Geometric Distortion}

The tomographic assumption of planar wavefronts leads to a geometric distortion of the image as well as the more troublesome space-variant defocus discussed here. As is discussed in Chapter 3, the geometric distortion, based on the presence of extraneous linear phase terms arising from wavefront curvature, is more prominent than the space-variant defocus yet is easily compensated via a simple post-warping procedure. The dominance of this distortion relative to defocus often necessitates the geometric post-warping procedure for many imaging scenarios not exhibiting quadratic defocus effects. Consequently, post-warping has long been considered a standard processing step of PFA, and the post-warping computation time is factored into the overall processing time of PFA. Fortunately, the post-warping procedure is based on a simple bilinear interpolation scheme which is not so computationally burdensome as to render PFA impractical.

The space-variant post-filtering step for image refocus is performed before the final geometric-post warping procedure. However, proper refocus depends on filter coefficients calculated using the point at the patch center, $\left(x_{0}^{\prime}, y_{0}^{\prime}\right)$, which is the spatial coordinate of the geometrically correct target position. That is, while the image has not yet been geometrically rewarped, proper refocus requires a priori knowledge of the final, post-warped (proper) position of that point in the slant plane. Based on this requirement, one might propose to rewarp the image before applying the space-variant post-filter for wavefront curvature correction. However, this approach presents a problem in and of itself. The post-warping procedure does not simply translate the points to their correct position, but instead uncurls them, based on the degree of wavefront curvature at that point. This is because the warping function is a continuous, space-variant function. Consequently, geometrically rewarping the image serves to rotate the targets (and their IPR sidelobes) by an amount equal to that required to rotate a tangent to the spherical wavefront at that point to be parallel with the Cartesian cross-range $\left(x^{\prime}\right)$ axis. 
Thankfully, based on the closed-form linear phase equations of Chapter 4, the induced geometric distortion can be calculated (and compensated) for all spatial coordinates in the slant plane, prior to applying the space-variant refocus filter. Once refocused, the post-warping procedure can be applied to the image, as prescribed by the polar-format algorithm. Recall Equation (4.12) from Chapter 4, which describes the total linear phase $\phi_{1 t}$ for a target in the slant plane at $\left(x_{0}^{\prime}, y_{0}^{\prime}\right)$ :

$$
\phi_{1 t}=x_{0}^{\prime} X^{\prime}+y_{0}^{\prime} Y^{\prime}+f_{\hat{x}}\left(x_{0}^{\prime}, y_{0}^{\prime}\right) X^{\prime}+f_{\hat{y}}\left(x_{0}^{\prime}, y_{0}^{\prime}\right) Y^{\prime},
$$

where $f_{\hat{x}}\left(x_{0}^{\prime}, y_{0}^{\prime}\right) X^{\prime}$ and $f_{\hat{y}}\left(x_{0}^{\prime}, y_{0}^{\prime}\right) Y^{\prime}$ define the extraneous phase terms in cross-range and range, respectively, in the presence of wavefront curvature. The effect of the extraneous phase terms is to distort (warp) a point $p_{0}^{\prime}$ correctly residing on the Cartesian grid at $\left(x_{0}^{\prime}, y_{0}^{\prime}\right)$, to an improper, wavefront curvature-induced position $p_{f}^{\prime}=$ $\left(x_{f}^{\prime}, y_{f}^{\prime}\right)$ on the polar grid. This situation is illustrated for one specific point in Figure 5.5. The linear shift property of the Fourier transform dictates that the linear phase coefficients of Equation (5.8) specify actual distances in the spatial image domain, and that specifically, the coefficients $f_{\hat{x}}\left(x_{0}^{\prime}, y_{0}^{\prime}\right)$ and $f_{\hat{y}}\left(x_{0}^{\prime}, y_{0}^{\prime}\right)$ relate to the offset distances induced by wavefront curvature. Consequently, the target positions $p_{0}^{\prime}$ and $p_{f}^{\prime}$ in Figure 5.5 can be described as follows:

$$
\begin{aligned}
p_{f}^{\prime} & =\left(x_{0}^{\prime}, y_{0}^{\prime}\right)+\left(f_{\hat{x}}\left(x_{0}^{\prime}, y_{0}^{\prime}\right), f_{\hat{y}}\left(x_{0}^{\prime}, y_{0}^{\prime}\right)\right) \\
& =p_{0}^{\prime}+\left(f_{\hat{x}}\left(x_{0}^{\prime}, y_{0}^{\prime}\right), f_{\hat{y}}\left(x_{0}^{\prime}, y_{0}^{\prime}\right)\right),
\end{aligned}
$$

where $p_{0}^{\prime}$ and $p_{f}^{\prime}$ are the correct and distorted positions, respectively. Thus, the distorted (warped) position of a point is simply the geometrically correct position $p_{0}^{\prime}$ offset by the linear displacement due to wavefront curvature and is specified by the following Cartesian ordered pair:

$$
\begin{aligned}
\left(x_{f}^{\prime}, y_{f}^{\prime}\right) & =p_{f}^{\prime} \\
& =\left(x_{0}^{\prime}+f_{\hat{x}}\left(x_{0}^{\prime}, y_{0}^{\prime}\right), y_{0}^{\prime}+f_{\hat{y}}\left(x_{0}^{\prime}, y_{0}^{\prime}\right)\right)
\end{aligned}
$$

A problem arises in the calculation of $\left(x_{0}^{\prime}, y_{0}^{\prime}\right)$ using Equation (5.11). Specifically, this equation describes the inverse of the situation encountered in space-variant post 


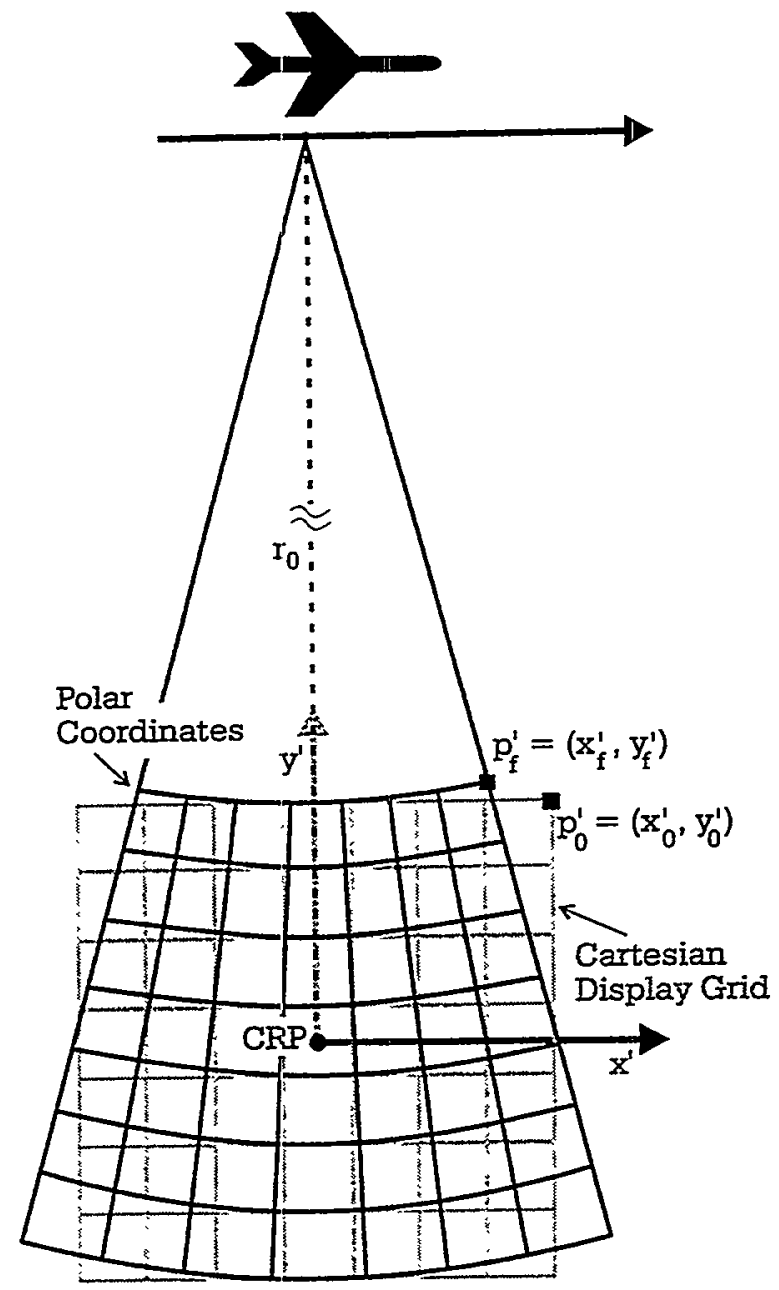

Figure 5.5: Effect of Geometric Distortion on Target Position

filtering. When post-filtering, one must determine (and derive the filter values for) the geometrically correct coordinate $p_{0}^{\prime}=\left(x_{0}^{\prime}, y_{0}^{\prime}\right)$ from the given warped coordinate $p_{f}^{\prime}=\left(x_{f}^{\prime}, y_{f}^{\prime}\right)$. This requires the inversion of Equation (5.11) such that it specifies the correct coordinate as a function of the warped coordinate. Rearranging Equation (5.9) yields

$$
\left(x_{0}^{\prime}, y_{0}^{\prime}\right)=p_{f}^{\prime}-\left(f_{\hat{x}}\left(x_{0}^{\prime}, y_{0}^{\prime}\right), f_{\hat{y}}\left(x_{0}^{\prime}, y_{0}^{\prime}\right)\right)
$$


and consequently,

$$
\begin{aligned}
\left(x_{0}^{\prime}, y_{0}^{\prime}\right) & =p_{0}^{\prime} \\
& =\left(x_{f}^{\prime}, y_{f}^{\prime}\right)-\left(f_{\hat{x}}\left(x_{0}^{\prime}, y_{0}^{\prime}\right), f_{\hat{y}}\left(x_{0}^{\prime}, y_{0}^{\prime}\right)\right)
\end{aligned}
$$

Finally, Equation (5.13) can be stated as the Cartesian ordered pair

$$
\begin{aligned}
\left(x_{0}^{\prime}, y_{0}^{\prime}\right) & =p_{0}^{\prime} \\
& =\left(x_{f}^{\prime}-f_{\hat{x}}\left(x_{0}^{\prime}, y_{0}^{\prime}\right), y_{f}^{\prime}-f_{\hat{y}}\left(x_{0}^{\prime}, y_{0}^{\prime}\right)\right)
\end{aligned}
$$

which is the inverse of Equation (5.11), as desired. This equation can then be used to determine the geometrically correct coordinate $x_{0}^{\prime}$ for a point $x_{f}^{\prime}$ that has undergone geometric displacement due to wavefront curvature, and subsequently, $x_{0}^{\prime}$ is used to determine the linear phase error at that point, according to Equation (5.1).

The Equation given by (5.14) is a recurrence relation. This recurrence is due to the coordinate $\left(x_{0}^{\prime}, y_{0}^{\prime}\right)$ being present on both sides of the equation. An iterative approach can used to solve this recurrence. Recall that the values $f_{\hat{x}}\left(x_{0}^{\prime}, y_{0}^{\prime}\right)$ and $f_{\hat{y}}\left(x_{0}^{\prime}, y_{0}^{\prime}\right)$ are the coefficients describing the linear displacement of the point from its proper position $\left(x_{0}^{\prime}, y_{0}^{\prime}\right)$ when wavefront curvature is present. Furthermore, the values of these coefficients, which directly infer a displacement distance, are also a function of the proper target position $\left(x_{0}^{\prime}, y_{0}^{\prime}\right)$. The recurrence can be approximately solved by using an estimate of the displacement which is based on the warped coordinate $p_{f}^{\prime}=\left(x_{f}^{\prime}, y_{f}^{\prime}\right)$ instead of the proper coordinate $\left(x_{0}^{\prime}, y_{0}^{\prime}\right)$, for which we are trying to solve:

$$
\left(x_{0}^{\prime}, y_{0}^{\prime}\right) \approx\left(x_{f}^{\prime}-f_{\hat{x}}\left(x_{f}^{\prime}, y_{f}^{\prime}\right), y_{f}^{\prime}-f_{\hat{y}}\left(x_{f}^{\prime}, y_{f}^{\prime}\right)\right)
$$

This approximation of $\left(x_{0}^{\prime}, y_{0}^{\prime}\right)$ will become more accurate as successive iterations are performed, with each using the previously derived $\left(x_{0}^{\prime}, y_{0}^{\prime}\right)$ value for the next calculation of $f_{\hat{x}}$ and $f_{\hat{y}}$. This concept can be generalized and stated in the following 
iterative form:

Let: $\quad\left(x_{1}^{\prime}, y_{1}^{\prime}\right)=\left(x_{f}^{\prime}, y_{f}^{\prime}\right)$.

Then: $\quad\left(x_{2}^{\prime}, y_{2}^{\prime}\right) \approx\left(x_{f}^{\prime}-f_{\hat{x}}\left(x_{1}^{\prime}, y_{1}^{\prime}\right), y_{f}^{\prime}-f_{\hat{y}}\left(x_{1}^{\prime}, y_{1}^{\prime}\right)\right)$,

$$
\begin{gathered}
\left(x_{3}^{\prime}, y_{3}^{\prime}\right) \approx\left(x_{f}^{\prime}-f_{\hat{x}}\left(x_{2}^{\prime}, y_{2}^{\prime}\right), y_{f}^{\prime}-f_{\hat{y}}\left(x_{2}^{\prime}, y_{2}^{\prime}\right)\right), \\
\vdots \\
\left(x_{n}^{\prime}, y_{n}^{\prime}\right) \approx\left(x_{f}^{\prime}-f_{\hat{x}}\left(x_{n-1}^{\prime}, y_{n-1}^{\prime}\right), y_{f}^{\prime}-f_{\hat{y}}\left(x_{n-1}^{\prime}, y_{n-1}^{\prime}\right)\right),
\end{gathered}
$$

where $\left(x_{n}^{\prime}, y_{n}^{\prime}\right)$ is a successively better approximation of the "proper" point $p_{0}^{\prime}=$ $\left(x_{0}^{\prime}, y_{0}^{\prime}\right)$, to which it eventually converges. Or, more compactly,

$$
\left(x_{n}^{\prime}, y_{n}^{\prime}\right)= \begin{cases}\left(x_{f}^{\prime}, y_{f}^{\prime}\right) & \text { if } n=1, \\ \left(x_{f}^{\prime}-f_{\hat{x}}\left(x_{n-1}^{\prime}, y_{n-1}^{\prime}\right), y_{f}^{\prime}-f_{\hat{y}}\left(x_{n-1}^{\prime}, y_{n-1}^{\prime}\right)\right) & \text { if } n>1,\end{cases}
$$

where $n$ is the number of iterations required such that $\left(x_{n}^{\prime}, y_{n}^{\prime}\right)$ is sufficiently close to the proper coordinate $p_{0}^{\prime}=\left(x_{0}^{\prime}, y_{0}^{\prime}\right)$. It is unknown as to whether a closed-form solution exists for Equation (5.17), and of course this solution would depend on the values of $f_{\hat{x}}$ and $f_{\hat{y}}$. Fortunately, it is not necessary to find a closed-form solution because in practice, only a few iterations are required to calculate the undistorted location $p_{0}^{\prime}$ for each filter center coordinate $p_{f}^{\prime}$. Furthermore, this coordinate is not calculated for each pixel location in the image, but instead only once for each patch center. Consequently, the computational burden associated with this geometric distortion compensation calculation is negligible.

One caveat remains in the geometric distortion compensation of Equation (5.17). As was mentioned in Chapter 4 in Sections 4.3.2 and 4.4.2, and described by Equation (5.8), the total linear phase is represented as a sum of linear phase terms. These terms include the phase components $x_{0}^{\prime} X^{\prime}$ and $y_{0}^{\prime} Y^{\prime}$, which serve to properly place the point in the spatial domain, as well as the extraneous terms $f_{\hat{x}}\left(x_{0}^{\prime}, y_{0}^{\prime}\right) X^{\prime}$ and $f_{\hat{y}}\left(x_{0}^{\prime}, y_{0}^{\prime}\right) Y^{\prime}$, which distort the point from its proper position in cross-range and range, respectively. For example, the geometric distortion for the broadside, small 
patch approximation is given by Equation (4.88) and repeated here:

$$
\begin{aligned}
\phi_{1 t} & =x_{0}^{\prime} X^{\prime}+y_{0}^{\prime} Y^{\prime}+f_{\hat{x}^{\prime}} X^{\prime}+f_{\tilde{y}^{\prime}} Y^{\prime} \\
& \approx x_{0}^{\prime} X^{\prime}+y_{0}^{\prime} Y^{\prime}+\frac{x_{0}^{\prime} y_{0}^{\prime}}{r_{0}} X^{\prime}-\frac{x_{0}^{\prime 2}}{2 r_{0}} Y^{\prime}, \quad x_{0}^{\prime}, y_{0}^{\prime} \ll r_{0},
\end{aligned}
$$

where $\frac{x_{0}^{\prime} y_{0}^{\prime}}{r_{0}} X^{\prime}$ and $-\frac{x_{0}^{\prime 2}}{2 r_{0}} Y^{\prime}$ denote the cross-range and range geometric distortion (error) phase terms $f_{\hat{x}^{\prime}} X^{\prime}$ and $f_{\hat{y}^{\prime}} Y^{\prime}$, respectively. This equation has the linear form consisting of the separable phase components, as specified by Equation (5.8). Consequently, the geometrically proper point $\left(x_{0}^{\prime}, y_{0}^{\prime}\right)$ can be calculated from the recurrence relation given by Equation (5.15), where $p_{f}^{\prime}=\left(x_{f}^{\prime}, y_{f}^{\prime}\right)$ is the coordinate of the patch center within the warped image being refocused. Furthermore, $f_{\hat{x}}\left(x_{0}^{\prime}, y_{0}^{\prime}\right)=\frac{x_{0}^{\prime} y_{0}^{\prime}}{r_{0}}$ and $f_{\hat{y}}\left(x_{0}^{\prime}, y_{0}^{\prime}\right)=-\frac{x_{0}^{\prime 2}}{2 r_{0}}$ define the geometric offset distances from $\left(x_{0}^{\prime}, y_{0}^{\prime}\right)$ in cross-range and range, respectively, due to wavefront curvature.

As another example, consider the exact linear phase error equation that represents all squint angles, first given by Equation (4.126) of Chapter 4:

$$
\begin{aligned}
\phi_{1 t} & =a_{10} X^{\prime}+a_{01} Y^{\prime} \\
& =\frac{r_{o}}{r_{c}}\left\{\tan \theta_{s}\left[r_{c}-r_{0}+y_{0}^{\prime}\right]+x_{0}^{\prime}\right\} X^{\prime}+\left(r_{0}-r_{c}\right) Y^{\prime},
\end{aligned}
$$

where $\theta_{s}$ specifies the mid-aperture squint angle measured from broadside, and $r_{c}$ is the distance from target to platform at mid-aperture. In this form, there appears no straightforward method for the decomposition of Equation (5.19) into its proper and linear distortion components, as specified by Equation (5.8). Instead it is in the form

$$
\phi_{1 t}=a_{10_{\left(x_{0}^{\prime}, y_{0}^{\prime}\right)}} X^{\prime}+a_{01_{\left(x_{0}^{\prime}, y_{0}^{\prime}\right)}} Y^{\prime}
$$

where the coefficients $a_{10_{\left(x_{0}^{\prime}, y_{0}^{\prime}\right)}}$ and $a_{01_{\left(x_{0}^{\prime}, y_{0}^{\prime}\right)}}$ describe the warped location $p_{f}^{\prime}$ of the point properly residing at $p_{0}^{\prime}=\left(x_{0}^{\prime}, y_{0}^{\prime}\right)$ in cross-range and range, respectively. This form was first given in Equation (4.47) of Chapter 4. In this case, the geometric displacement (error) terms are not separate from those of $x_{0}^{\prime}$ and $y_{0}^{\prime}$. Thus, the approach currently being discussed, using the relation of Equation (5.15), can not 
be used to determine the geometrically correct position of the patch center. Still, it is necessary to calculate the inverse of this relation; that is, to determine the point $p_{0}^{\prime}=\left(x_{0}^{\prime}, y_{0}^{\prime}\right)$ given the point $\left(x_{f}^{\prime}, y_{f}^{\prime}\right)$ in the warped image.

An alternative method must be devised for calculating the geometrically correct point $\left(x_{0}^{\prime}, y_{0}^{\prime}\right)$ given the more complicated linear phase expression of Equation ( 5.19$)$. To this end, consider the following mathematical "trick," which refers to the coordinates depicted in Figure 5.5. By the linear phase shift property of the Fourier transform, the resultant, geometrically warped location $p_{f}^{\prime}$ for the properly placed point $p_{0}^{\prime}=\left(x_{0}^{\prime}, y_{0}^{\prime}\right)$ is given by the Cartesian expression:

$$
\begin{aligned}
& p_{f}^{\prime}=\left(x_{f}^{\prime}, y_{f}^{\prime}\right)
\end{aligned}
$$

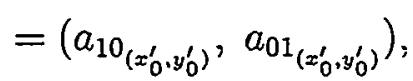

where the terms $a_{10}$ and $a_{01}$ are the linear Taylor series coefficients as derived in Chapter 4, Section 4.3.1. This development is based on the understanding that the extraneous linear phase terms defining the offset from $\left(x_{0}^{\prime}, y_{0}^{\prime}\right)$ cannot easily be isolated. Thus, this development does not rely on the separability of the extraneous linear phase error terms from the proper terms, but instead considers $p_{f}^{\prime}$ as the inseparable combination of these terms. First, let

$$
\begin{aligned}
p_{f}^{\prime} & =-p_{0}^{\prime}+\left(p_{f}^{\prime}\right)+p_{0}^{\prime} \\
& =-\left(x_{0}^{\prime}, y_{0}^{\prime}\right)+\left(p_{f}^{\prime}\right)+p_{0}^{\prime}
\end{aligned}
$$

where $p_{0}^{\prime}$ specifies the proper coordinate for the point warped to $p_{f}^{\prime}$. Then, from Equation (5.21),

$$
p_{f}^{\prime}=-\left(x_{0}^{\prime}, y_{0}^{\prime}\right)+\left(a_{10_{\left(x_{0}^{\prime}, y_{0}^{\prime}\right)}}, a_{01_{\left(x_{0}^{\prime}, y_{0}^{\prime}\right)}}\right)+p_{0}^{\prime}
$$


Rearranging yields

$$
\begin{aligned}
p_{0}^{\prime} & =p_{f}^{\prime}+\left(x_{0}^{\prime}, y_{0}^{\prime}\right)-\left(a_{10_{\left(x_{0}^{\prime}, y_{0}^{\prime}\right)}, a_{01}}{ }_{\left(x_{0}^{\prime}, y_{0}^{\prime}\right)}\right) \\
& =\left(x_{f}^{\prime}, y_{f}^{\prime}\right)+\left(x_{0}^{\prime}, y_{0}^{\prime}\right)-\left(a_{10_{\left(x_{0}^{\prime}, y_{0}^{\prime}\right)}, a_{01}}\right) \\
& =\left(\left(x_{f}^{\prime}+x_{0}^{\prime}, y_{0}^{\prime}\right)\right. \\
& =\left(x_{0}^{\prime}, y_{0}^{\prime}\right) .
\end{aligned}
$$

This recurrence relation specifies the proper coordinate $p_{0}^{\prime}$ for the filter calculation, based on the patch center given by $p_{f}^{\prime}$ in the warped image. As was the case previously, this recurrence can be solved by the following iteration sequence:

Let: $\left(x_{1}^{\prime}, y_{1}^{\prime}\right)=\left(x_{f}^{\prime}, y_{f}^{\prime}\right)$.

Then: $\quad\left(x_{2}^{\prime}, y_{2}^{\prime}\right) \approx\left(\left(x_{f}^{\prime}+x_{1}^{\prime}-a_{\left.10_{\left(x_{1}^{\prime}, y_{1}^{\prime}\right.}\right)}\right),\left(y_{f}^{\prime}+y_{1}^{\prime}-a_{\left.\left.01_{\left(x_{1}^{\prime}, y_{1}^{\prime}\right)}\right)\right)}\right.\right.$

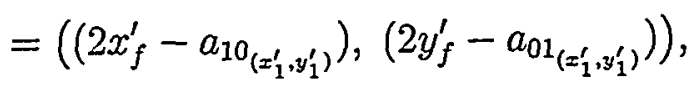

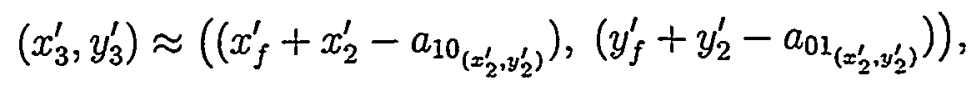

$$
\begin{aligned}
& \left(x_{n}^{\prime}, y_{n}^{\prime}\right) \approx\left(\left(x_{f}^{\prime}+x_{n-1}^{\prime}-a_{10_{\left(x_{n-1}^{\prime}, y_{n-1}^{\prime}\right)}}\right),\left(y_{f}^{\prime}+y_{n-1}^{\prime}-a_{\left.01_{\left(x_{n-1}^{\prime}, y_{n-1}^{\prime}\right)}\right)}\right)\right.
\end{aligned}
$$

where $\left(x_{n}^{\prime}, y_{n}^{\prime}\right)$ is a successively better approximation of the "proper" point $p_{0}^{\prime}=$ $\left(x_{0}^{\prime}, y_{0}^{\prime}\right)$, to which it eventually converges. Or, more compactly,

$$
\left(x_{n}^{\prime}, y_{n}^{\prime}\right)= \begin{cases}\left(x_{f}^{\prime}, y_{f}^{\prime}\right) & \text { if } n=1 \\ \left(\left(x_{f}^{\prime}+x_{n-1}^{\prime}-a_{10}{\left(x_{n-1}^{\prime}, y_{n-1}^{\prime}\right)}^{\prime}\right),\left(y_{f}^{\prime}+y_{n-1}^{\prime}-a_{\left.\left.01_{\left(x_{n-1}^{\prime}, y_{n-1}^{\prime}\right)}\right)\right)}\right.\right. & \text { if } n>1\end{cases}
$$

Again, this sequence quickly converges to a very accurate representation of $p_{0}^{\prime}=$ $\left(x_{0}^{\prime}, y_{0}^{\prime}\right)$. For the generalized squint-mode scenario of Equation (5.19), this recurrence 
becomes

$$
\left(x_{n}^{\prime}, y_{n}^{\prime}\right)= \begin{cases}\left(x_{f}^{\prime}, y_{f}^{\prime}\right) & \text { if } n=1 \\ \left(\left(x_{f}^{\prime}+x_{n-1}^{\prime}-\frac{r_{0}}{r_{c_{n-1}}}\left\{\tan \theta_{s}\left[r_{c_{n-1}}-r_{0}+y_{n-1}^{\prime}\right]+x_{n-1}^{\prime}\right\}\right),\right. & \\ \left(y_{f}^{\prime}+y_{n-1}^{\prime}-\left(r_{0}-r_{c_{n-1}}\right)\right) & \text { if } n>1\end{cases}
$$

where $\left(x_{f}^{\prime}, y_{f}^{\prime}\right)$ is at the center of the patch being refocused (in the warped domain), and from Equation (4.67),

$$
r_{c_{n}}=\sqrt{r_{0}^{2}+x_{n}^{\prime 2}+y_{n}^{\prime 2}-2 r_{0} y_{n}^{\prime}}
$$

given the slant range from platform at mid-aperture to scene center of $r_{0}$.

\subsection{Filter Overlap and Width}

The computational burden of space-variant post-filtering is reduced by varying the filter only as often as necessary. In other words, the filter is applied to as large a patch as possible, but not so large as to degrade the quality of refocus. For each patch, the corrective quadratic phase function is calculated for the patch center $\left(x_{0}^{\prime}, y_{0}^{\prime}\right)$ according to Equation (5.1), or its simplified approximations, if applicable. However, the patch center coordinate is inst geometrically corrected to account for wavefront curvature, as described in Section 5.3. Since the filter is not applied to every spatial location in the image but instead across a given patch, the filter serves to exactly compensate the wavefront curvature at the center point $\left(x_{0}^{\prime}, y_{0}^{\prime}\right)$, but is only an approximate correction to those points surrounding the center. Points farther from the center of the patch are not as well focused as those nearer the middle; therefore, the patch size must be restricted to some maximum size $m$ (and this size varies with filter location within the image). Furthermore, it is important for adjacent filter operations to overlap each other in the image domain as to ensure the entire spatial blur (broadened IPR) is encompassed by a given filter or one of its immediately 
adjacent filter applications. This process is illustrated in Figure 5.4. In this way, having provided the required minimum filter overlap $c$, an IPR will be correctly focused, independent of the IPR's position in the image and without regard to the relative position of the IPR to filter. In other words, this overlap ensures proper refocus over the continuum of point targets in the imaged scene, based on a filter that is applied at discrete steps. The filter displacement $d$ is the lateral movement of the filter's center in cross-range from one filter application to the next. It is simply the difference between the filter length and the overlap. The relationship between overlap, length, and displacement is illustrated in Figure 5.3.

How does one go about determining the appropriate filter overlap, length and displacement for a given imaging scenario? First, consider filter overlap. The overlapping region of adjacent filter applications must be sufficiently wide in cross-range to cover the spatial extent of the blur. This blur can be described as a broadening of the IPR, and is based on the amount of Fourier domain quadratic phase at that point in the image. The effect of quadratic phase is to broaden the IPR, in proportion to the amount of peak quadratic phase present. This situation is illustrated in Figure 3.8 of Chapter 2, for various amounts of induced quadratic phase error. The mathematical development of Appendix A serves to provide an analytical, closedform expression for determining the IPR broadening, as a function of peak quadratic phase (magnitude of phase at the edge of the signal). The reader is encouraged to study the IPR broadening argument of Appendix A, which provides both a frequency and spatial domain analysis, each coming to the same conclusion. However, in this chapter, only the results of this analysis will be presented. Based on the analysis of Appendix A, the IPR mainlobe broadening factor $\Delta_{I P R}$, also referred to the pixel spread value $\Delta n_{\epsilon}$, is found to be

$$
\Delta_{I P R}=\frac{4\left|\phi_{e}\right|}{\pi}
$$

where $\phi_{\epsilon}$ is the maximum quadratic phase as taken at the edge of the frequency extent $X_{1}^{\prime}$, where $\left|X^{\prime}\right| \leq X_{1}^{\prime}$, and $X^{\prime}$ is given by Equation (5.3). The quadratic phase $\phi_{x_{0}^{\prime}, y_{0}^{\prime}}\left(X^{\prime}\right)$ is space-variant and is given by the quadratic phase error Equations (5.1), 
(5.4) and (5.5), depending on the imaging scenario and amount of approximation that is acceptable. Then, based on Equation (5.29), the minimum amount of overlap $c$, in pixels, is defined such that

$$
c \geq \frac{4\left|\phi_{x_{0}^{\prime}, y_{0}^{\prime}}\left(X_{1}^{\prime}\right)\right| K_{a} o_{x}}{\pi}, \quad \text { (pixels) }
$$

where $K_{a}$ is the IPR mainlobe broadening factor due to windowing (sidelobe control) and $o_{x}$ is the oversample factor in cross-range.

For example, one might calculate the minimum required overlap $c$ at the edge of the L-Band patch described by table 5.1. Specifically, assume that an overlap between adjacent filter operations occurs at the position $\left(x_{0}^{\prime}, y_{0}^{\prime}\right)=(1000,0)$. This spatial location, along the $x^{\prime}$ axis at the far scene edge in cross-range, is known to exhibit significant defocus due to wavefront curvature. The Fourier domain frequency extent is found to be in the range $\left[-X_{1}^{\prime} . . X_{1}^{\prime}\right]$, where $X^{\prime}=\left(\pi / \rho_{x}\right)=(3.1415 / 1.25) \approx 2.5$ $\mathrm{rad} / \mathrm{m}$, and $\left|X^{\prime}\right| \leq X_{1}^{\prime}$. Thus, the frequency extent at the quadratic signal edge is $X_{1}^{\prime 2} \approx(2.5)^{2}=6.25$. Equation (5.1) will be used to calculate the maximum quadratic phase error at this point. This is the generalized equation which gives the exact phase for all patch sizes, ranges, squint angles and center frequencies. When substituting the value $\left(X_{1}^{\prime 2}=6.25\right)$ for $X^{\prime 2}$ in Equation (5.1), this calculation yields a peak quadratic phase error $\phi_{2 t}$ of $3.6 \pi$ radians, where $\left(x_{0}^{\prime}, y_{0}^{\prime}\right)=(1000,0)$, $r_{0}=4972 \mathrm{~m}, \theta_{s}=0^{\circ}$ (broadside), $k_{0}=(4 \pi / \lambda) \approx 52.4$, and $r_{c}\left(x_{0}^{\prime}, y_{0}^{\prime}, r_{0}\right)=5071$ (from Equation (5.28)). It is interesting to note that if the small patch assumption is applied, then the approximation of Equation (5.5) yields a peak quadratic phase error of $\phi_{2 t}=3.8 \pi$, an error of about six percent from the actual value. Thus, this approximation is not appropriate in this case. However, it is known a priori not to apply this approximation, since $x_{0}^{\prime}, y_{0}^{\prime} \nless r_{0}$.

Based on the peak quadratic phase error $\phi_{2 t}=3.6 \pi$ just calculated for the L-Band example, the IPR broadening factor can now be calculated, and subsequently, the filter overlap at $\left(x_{0}^{\prime}, y_{0}^{\prime}\right)=(1000,0)$ can be determined. The broadening factor $\triangle_{I P R}$ is based on the analysis of Appendix $A$ and the simple result of Equation (5.29). From 
this equation and the quadratic phase error $\phi_{2 t}$ just calculated, $\Delta_{I P R}=\left(4 \phi_{2 t} / \pi\right)=$ $(4 \times 3.6)=14.4$. This is a dimensionless broadening ratio based on the ideal IPR width in the processed image. The ideal IPR width is determined by many factors in real imagery but in the ideal case, is based on the theoretical image resolution and the pixel scale factor (which in this situation, accounts for the specific oversample ratio of the image). For the L-Band parameters of Table 5.1, the ideal cross-range IPR width, in pixels, is found to be $\left(\rho_{x} / s_{x}\right)=(1.25 / 0.65)=1.92$, which is the oversample value $o_{x}$. Multiplied by the broadening factor $\triangle_{I P R}=14.4$ yields a cross-range blur of $(14.4 \times 1.92) \approx 28$ pixels. Additionally, there is another broadening factor, $K_{a}$, which represents the widening of the IPR mainlobe due to Taylor filtering. In this case, $K_{a}=1.4$, so the maximum defocus is $(28 \times 1.4) \approx 40$ pixels. Thus, the refocus filter overlap $c$ at this spatial location in the image must be at least 40 pixels, as prescribed by Equation (5.30). As will be illustrated in Chapter 6, there is additional computational cost, yet no improvement in quality of refocus when this overlap is increased. However, should the minimum limit on the overlap be violated, refocus can not be achieved, and in fact targets will become very distorted once the filter is applied. The overlap determination is based entirely on analytical, closed form calculations. Consequently, it is a simple matter to incorporate these calculations into a computer algorithm that calculates overlaps on an as-needed basis. Furthermore, the computation time for these calculations is negligible compared to that of the filtering operation.

Having calculated the appropriate filter overlap, the filter length may now be considered. In practice, these calculations are independent and may be done in either order. The filter length is, in essence, dictated by patch width, in crossrange, to which the filter is applied. Since the refocus filter exactly compensates only the center pixel of the patch, the patch size must be constrained so as to limit the residual quadratic phase error at the edges of the patch upon refocus. In this way, the IPR broadening is constrained to within sub-pixel levels across the patch, and consequently, the residual defocus after filtering is visually imperceptible. The 
calculation of maximum patch size is similar to that for maximum scene size, as described in Section 3.3 of Chapter 3. However, in the case of overall scene size, the reference point is alwrays the center reference point (CRP), where $\left(x_{0}^{\prime}, y_{0}^{\prime}\right)=(0,0)$. In contrast, the maximum patch size for a filter application is space-variant as a function of filter center $\left(x_{0}^{\prime}, y_{0}^{\prime}\right)$. Thus, it is convenient to calculate filter length based on a formula for the rate of change of the quadratic phase at the filter center. Then, by knowing this rate of change, the spatial distance (length) of the filter can be determined so as to maintain a maximum acceptable residual quadratic phase error at the farthest-most patch edges in cross-range.

As was the case with the overall scene size calculations, the residual quadratic phase error is to be held to within $\pi / 4$ radians in order to constrain the residual defocus to sub-pixel levels. Once having calculated the rate of change of quadratic phase error at the filter center, it is a simple task to determine if the filter extends beyond the point where the applied phase correction is not sufficient to adequately compensate (to within $\pi / 4 \mathrm{rad}$ ) the far-edge points. The quadratic phase error equation of (5.5) will be used for the rate of change calculation. While this equation serves as a small patch approximation to the actual phase error, it is sufficiently accurate for use as a patch size estimator, at least for most imaging scenarios. The rate of change of the quadratic phase error in cross-range is of the following general form:

$$
\dot{\phi}_{\left(x_{0}^{\prime}, y_{0}^{\prime}\right)}\left(X^{\prime}, Y^{\prime}\right)=\frac{\partial \phi_{\left(x_{0}^{\prime}, y_{0}^{\prime}\right)}\left(X^{\prime}, Y^{\prime}\right)}{\partial x_{0}^{\prime}} .
$$

From Equation (5.5),

$$
\begin{aligned}
\dot{\phi}_{\left(x_{0}^{\prime}, y_{0}^{\prime}\right)}\left(X^{\prime}, Y^{\prime}\right) & =\frac{\partial\left[\frac{1}{2}\left\{\frac{x_{0}^{\prime 2}-y_{0}^{\prime 2}}{r_{0} k_{0}}\right\} X^{\prime 2}\right]}{\partial x_{0}^{\prime}} \\
& =\frac{x_{0}^{\prime}}{r_{0} k_{0}} X^{\prime 2} .
\end{aligned}
$$

The partial derivative is taken with respect to $x_{0}^{\prime}$ because we are concerned with the rate of change in cross-range. Furthermore, the blur is one-dimensional in cross-range 
only, and the maximum frequency extent of the aperture $X_{1}^{\prime}$ must be considered, where $\left|X^{\prime}\right| \leq X_{1}^{\prime}$. Thus, Equation (5.33) is more appropriately stated as

$$
\dot{\phi}_{\left(x_{0}^{\prime}\right)}\left(X_{1}^{\prime}\right)=\frac{x_{0}^{\prime}}{r_{0} k_{0}} X_{1}^{\prime 2} .
$$

This rate of change in $\mathrm{rad} / \mathrm{m}$, referenced from the filter (patch) center $\left(x_{0}^{\prime}, y_{0}^{\prime}\right)$, is multiplied by some maximum patch radius $m_{r}$, yielding an overall change of phase from center to patch edge of no more than $\pi / 4$ radians. In this way, the residual defocus at the filter edge is maintained to within sub-pixel levels. This concept is described mathematically by the inequality

$$
\dot{\phi}_{\left(\mid x_{0}^{\prime}\right)}\left(X_{1}^{\prime}\right) \times m_{r} \leq \frac{\pi}{4} .
$$

Rearranging Equation (5.35), the maximum allowable patch radius $m_{r}$ in cross-range from the center filter position $\left(x_{0}^{\prime}, y_{0}^{\prime}\right)$ is found to be

$$
m_{r} \leq \frac{\pi}{4 \dot{\phi}_{\left(\left|x_{0}^{\prime}\right|\right)}\left(X_{1}^{\prime}\right)} .
$$

By substitution from Equation (5.34),

$$
m_{r} \leq \frac{\pi}{4\left[\frac{\left|x_{0}^{\prime}\right|}{r_{0} k_{0}} X_{1}^{\prime 2}\right]} .
$$

Rearranging,

$$
m_{r} \leq \frac{\pi r_{0} k_{0}}{4\left|x_{0}^{\prime}\right| X_{1}^{\prime 2}}
$$

where $m_{r}$ is the maximum patch radius. The relation may be also be expressed in terms of image resolution in cross-range, via substitution from Equation (5.3):

$$
m_{r} \leq \frac{\pi r_{0} k_{0}}{4\left|x_{0}^{\prime}\right|\left(\frac{\pi}{\rho_{x}}\right)^{2}}=\frac{\rho_{x}^{2} r_{0} k_{0}}{4 \pi\left|x_{0}^{\prime}\right|}
$$

The filter length $m$ is then twice the filter radius, or $2 m_{r}$. Furthermore, the distance is converted to pixels by the multiplication of the inverse scale factor, $1 / s_{x}$. Thus,

$$
m \leq \frac{\pi r_{0} k_{0}}{2 s_{x}\left|x_{0}^{\prime}\right| X_{1}^{\prime 2}}=\frac{\rho_{x}^{2} r_{0} k_{0}}{2 \pi s_{x}\left|x_{0}^{\prime}\right|} \quad \text { (pixels) }
$$


As would be expected, the maximum parch size that can be refocused necessarily decreases for reduced slant range $r_{0}$, reduced center frequency (decreasing $k_{0}$ ), improved resolution (increasing $X_{1}^{\prime}$ or decreasing $\rho_{x}$ ), and towards the far edges of the imaged scene in cross-range (increasing $x_{0}^{\prime}$ ). These are the scenarios that intensify the wavefront curvature effects under the planar wavefront assumption. These scenarios are consistent with those that increase the overall quadratic phase error as described by Equations (5.1), (5.4) and (5.5).

The maximum patch diameter $m$ (which is also the filter length) can now be calculated for the L-Band example. As before, the parameters for this example are given in Table 5.1. From Equation (5.40), the maximum patch radius for the filter applied at $\left(x_{0}^{\prime} y_{0}^{\prime}\right)=(1000,0)$ is $\left(1.25^{2} \times 4972 \times 52.4\right) /(2 \times \pi \times 1000) \approx 64 \mathrm{~m}$. The filter length in pixels is then found by taking the product of this value with the inverse scale factor: $m \times 1 / s_{x}=64 / 0.65 \approx 98$ pixels. Of course, the phase error function of Equation (5.5), which is quadratic as a function of the spatial target location $x_{0}^{\prime}$, implies a rate of change function that is also space-variant (though linear) with respect to $x_{0}^{\prime}$. This is obvious since the latter function is the derivative of the former. Thus, one must be cautious when applying the rate of change criteria for filter length across the image. For example, the rate of change of the quadratic phase error at the CRP is zero, yet a filter of full image length will not suffice to compensate the defocus. Consequently, one must "look ahead" when calculating the filter length at a given point, to ensure the filter is not too wide to adequately compensate the edgemost pixels, whose rate of change of quadratic phase is different than those nearer the center of the filter. For squinted scenarios, the above development for maximum patch radius $m_{r}$ may be repeated using the squint-mode Equation (5.4) instead of the broadside equation (5.5). 


\subsection{Filter Displacement}

The discussion of the previous section raises the issue of a potential incompatibility between filter displacement, overlap and length, as prescribed by Figure 5.3. In fact, the L-Band example being discussed brings to light this very serious contradiction. Specifically, the minimum filter overlap $c$ for the L-Band example was calculated from Equation (5.29) and found to be $c=40$ pixels. Meanwhile, the maximum patch diameter $m$ (which is twice the filter radius $m_{r}$ ) was determined by Equation (5.40) to be $m=98$ pixels. When implemented within a computer algorithm, typically a fixed-radix, fast Fourier Transform is implemented. In the case of the typical radix-2 algorithm, FFT lengths are always powers of two. Since the filter operation consists of an FFT of the patch, complex-conjugate multiply of the corrective quadratic phase function in the Fourier domain, and an inverse FFT of the refocused patch back into the spatial domain, the filter lengths are constrained to the FFT radix sizes. If a filter length of 98 is desired, then the next higher power of two is 128. However, this filter length is greater than the maximum allowed for proper image refocus! Fortunately, in this case, one may choose the next smaller power of two, which is 64 , and a filter length of $m=64$ is sufficient to provide the required minimum overlap of $c=40$ pixels. However, it does so just barely.

In many imaging scenarios, there will be certain $\left(x_{0}^{\prime}, y_{0}^{\prime}\right)$ coordinates in an imaged scene where the maximum allowable filter width is less than the minimum required overlap, and hence the contradiction. However, this conflict is easily resolved by slightly rethinking the meaning of "filter length" and tying this definition to filter displacement $d$ as opposed to filter length $m$. In this way, except for the possible higher computational burden associated with a longer filter, there will no longer be a maximum constraint on filter length. Instead, this maximum constraint is placed on filter displacement $d$. Hence, the contradiction will be solved for all imaging scenarios. This process is described as follows.

A filter's maximum length $m$ is defined as twice the distance from the filter 
center to the point at which pixels can no longer be adequately focused based on the filter parameters for that patch. However, a filter may need to be longer than this maximum in order to provide a sufficient amount overlap with the previous filter operation, so as to cover the spatial extent of the IPR blur. In this case, only the innermost $d$ bits (referenced from $\left(x_{0}^{\prime}, y_{0}^{\prime}\right)$ ) will be retained upon refocusing the patch. That is, the width of the refocused patch is controlled by retaining no more refocused pixels, after filtering a specific patch, than the maximum prescribed by Equation (5.40). So, let the displacement distance $d$ be constrained as follows:

$$
d \leq \frac{\pi r_{0} k_{0}}{2 s_{x}\left|x_{0}^{\prime}\right| X_{1}^{\prime 2}}=\frac{\rho_{x}^{2} r_{0} k_{0}}{2 \pi s_{x}\left|x_{0}^{\prime}\right|} \quad \text { (pixels) }
$$

Then, the filter length $m$ must be equal to $d+c$. That is;

$$
m=c+d
$$

where $c$ is the overlap, whose minimum is defined by Equation (5.30), and $d$ is the displacement, whose maximum is given by Equation (5.41). Thus, except for the increased computational burden associated with a larger overlap from one filter application to the next, there is no longer a maximum limit on filter length $m$. For this example, the maximum displacement $d$ is 98 pixels, and the minimum overlap $c$ is 40 pixels. As was described in Section 5.1 and illustrated in Figures 5.1 and 5.2, the chosen values of $c=64$ for overlap and $d=64$ for displacement $(m=128)$ have indeed turned out to be appropriate, based on the analysis of this section. Note, too, that the minimum displacement $d$ must be one pixel, to ensure "forward movement" of the filter from patch to patch. Equation (5.41) will yield (at least) this minimum displacement for all known imaging scenarios.

The filter is applied to successive patches across the image in cross-range, each separated from the previous by the displacement distance $d$. The innermost $d$ refocused pixels for each patch, as centered around $\left(x_{0}^{\prime}, y_{0}^{\prime}\right)$, are then copied to a new storage area representing the refocused image. In this way, each pixel will be focused once and only once. Storing the refocused pixels in a new memory area instead 
of in-place ensures the overlap operations are always performed on the prefocused Fourier data. The space-variant post-filter design of this chapter will be applied to the computational examples of Chapter 6 , which will be analyzed in terms of processing burden and quality of refocus. 
This page intentionally left blank 


\section{Chapter 6}

\section{Performance of the Space-Variant Post-Filter}

The previous two chapters presented a geometric model for the phase return of a point target, and subsequently, the phase errors arising from the faulty planar wavefront assumption were mathematically quantified. A unique contribution of this dissertation is the generalized phase return model that applies to all squint scenarios without relying on small patch approximations. This generalized model is an extension the broadside-only model originally presented in [7, pp. 355-365][10, 11], and Chapter 5 shows that the original broadside-only model is simply a specific case of the new, generalized model. Also in Chapter 5, the second-order phase error equation, derived from the generalized phase return model, was used as the basis for the space-variant post-filter phase correction factor. Furthermore, a corrective method (algorithm) was proposed for the efficient application of this filter to polarformatted imagery defocused by uncompensated wavefront curvature. This method is efficient in that it changes the filter only as often as necessary to constrain residual defocus to within subpixel levels. This is accomplished by determining an appropriate (minimum) filter overlap $c$, maximum displacement $d$, and filter length $m$, where $m=c+d$. The equations for $c, d$, and $m$, as described in the previous chapter, are also 
unique and important contributions of this dissertation, as is the iterative numerical method which accounts for geometric distortion during the refocus operation. Adding to the efficiency of this algorithm is the knowledge that the refocus filter remains one-dimensional, in cross-range only, even for squint-mode collection scenarios.

What remains to be explored is how well the space-variant post-filter works in practice, and how efficient it is (in conjunction with the polar-format algorithm), compared to other image formation algorithms. Thus, at this point, a number of questions arise regarding the performance of the space-variant post-filter. For example, how does filter size, displacement and overlap affect computational performance? How can these parameters be altered to better the computational efficiency, without affecting the quality of refocus? How does the computation time of space-variant post-filtering, in conjunction with polar-formatting, compare to other image formation methods, for both squinted and broadside collection scenarios? Finally, in practice, just how good is the quality of refocus, anyway? These questions will be addressed in this chapter. As will be shown, space-variant post-filtering provides an excellent solution to the wavefront curvature problem in polar-formatted spotlight mode imagery, both in terms of computational burden and quality of refocus, for both broadside and squinted collection geometries. Furthermore, even with the additional computational burden of space-variant post-filtering (which is typically as little as $30 \%$ of PFA time), no other competitive image formation algorithm shows a clear computational advantage over PFA.

\subsection{Quality of Refocus}

A number of issues have been discussed which lead one to believe that space-variant post-filtering does not perfectly compensate the phase errors (and consequent defocus) associated with wavefront curvature. Indeed, the post filter does not provide perfect compensation. However, it is hoped (and will be demonstrated here) that 
in most imaging scenarios, refocus is performed to within subpixel specifications, as prescribed by the residual quadratic phase error limit of $\pi / 4$ radians. That is, the refocus is "good enough," at least as perceived by the human eye. Recall from Chapter 4, Equations (4.47) and (4.48), the Taylor series expansion of the phase function $\phi_{x_{0}^{\prime}, y_{0}^{\prime}}\left(X^{\prime}, Y^{\prime}\right)$ expanded around the point $\left(X_{0}, Y_{0}\right)$ :

$$
\begin{aligned}
\phi_{x_{0}^{\prime}, y_{0}^{\prime}}\left(X^{\prime}, Y^{\prime}\right)= & a_{00}+a_{10} X^{\prime}+a_{01} Y^{\prime}+a_{20} X^{\prime 2} \\
& +a_{11} X^{\prime} Y^{\prime}+a_{02} Y^{\prime 2}+\ldots
\end{aligned}
$$

where

$$
a_{i j}=\left.\left(\frac{1}{i ! j !}\right)\left(\frac{\partial^{i+j} k\left(r_{x}-r_{t}\right)}{\partial X^{i} \partial Y^{j}}\right)\right|_{\substack{X=X_{0}, Y=Y_{0}}} .
$$

For the purpose of post-filtering for wavefront curvature correction, this expansion has been limited to the second-order, quadratic term in cross-range (remembering that the quadratic phase error terms in range are always zero). That is, the generalized quadratic phase error $\phi_{2 t}\left(X^{\prime}, Y^{\prime}\right)$ for all squint angles is simply

$$
\begin{aligned}
\phi_{2 t\left(x_{0}^{\prime}, y_{0}^{\prime}\right)}\left(X^{\prime}, Y^{\prime}\right) & =\phi_{2 t\left(x_{0}^{\prime}, y_{0}^{\prime}\right)}\left(X^{\prime}\right) \\
& =a_{20} X^{\prime 2}
\end{aligned}
$$

and by Equation (5.1),

$$
\begin{aligned}
\phi_{2 t\left(x_{0}^{\prime}, y_{0}^{\prime}\right)}\left(X^{\prime}\right)= & \frac{1}{2}\left\{\left[\frac{r_{0}-r_{c}}{k_{0}}-\frac{r_{0}\left(r_{0}-r_{c}\right)}{r_{c} k_{0}}+\frac{x_{0}^{\prime 2} r_{0}^{2}}{r_{c}^{3} k_{0}}\right]\right. \\
& +\frac{2 r_{0} \tan \theta_{s}}{r_{c} k_{0}}\left[x_{0}^{\prime}+\frac{x_{0}^{\prime} y_{0}^{\prime} r_{0}-x_{0}^{\prime} r_{0}^{2}}{r_{c}^{2}}\right] \\
& \left.+\frac{r_{0} \tan ^{2} \theta_{s}}{r_{c} k_{0}}\left[2 r_{c}-3 r_{0}+2 y_{0}^{\prime}+\frac{r_{0}^{3}-2 y_{0}^{\prime} r_{0}^{2}+y_{0}^{\prime 2} r_{0}}{r_{c}^{2}}\right]\right\} X^{\prime 2}
\end{aligned}
$$

where $k_{0}=\frac{4 \pi f_{0}}{c}=\frac{4 \pi}{\lambda}$ is the nominal phase history radius, $r_{0}$ is the slant range from platform to CRP at mid-aperture, $r_{c}$ is the distance from the platform at midaperture to the target positioned at $\left(x_{0}^{\prime}, y_{0}^{\prime}\right), \theta_{s}$ is the squint angle (measured from broadside), and $X^{\prime}$ is the cross-range frequency extent. 
By nature of the Taylor series expansion of Equations (6.1) and (6.2), the higherorder terms "fall off" (decay) quickly and hence, the quadratic term given by Equation (6.5) dominates. However, there still exist higher order phase terms in the Fourier space due to wavefront curvature. When analyzing the refocused point targets via their frequency response plots, there is evidence of the higher-order phase errors which serve to defocus the target. Fortunately, as expected, even for those systems most sensitive to wavefront curvature defocus, namely the L-Band and UWB SARs, this residual phase error does not induce blur that is visibly noticeable in the magnitude-detected image. This is true even of the highest resolution systems known to date. However, since post-filtering does not compensate higher than second-order wavefront curvature phase errors, and because radar system specifications are continually improving, it is safe to say that most imaging scenarios are adequately refocused, but perhaps not all. The quality of refocus for the most severe scenarios currently encountered, those in the L-Band frequency spectrum, are considered in the examples below.

First, consider the SYNTARG, squint-mode L-BAND patch, as shown in Figure 6.1. This figure depicts a scene of circularly arranged point targets, formed by the polar-formatting algorithm, before applying the new space-variant post-filter for wavefront curvature correction. This scenario represents a patch diameter $D_{p}$ of 2400 $\mathrm{m}$, a center frequency of $1.25 \mathrm{gHz}$ and a spatial resolution of $1.25 \mathrm{~m}$ in both range and cross range (see Table 5.1 for a complete list of parameters). In addition, the radar is squinted $30.5^{\circ}$ at mid-aperture towards the CRP. While the targets were originally placed in a perfectly circular pattern in the ground plane, the geometric distortion (into an irregular ellipsoid) resulting from the projection into the slant plane is readily apparent. There is a distortion in both the range and cross-range direction, due to a tilt in the slant plane induced by the depression angle and degree of squint. Fortunately, for the purpose of slant plane wavefront curvature correction, one need not be concerned with the distortion resulting from projecting the ground plane targets into the slant plane. Instead, the target coordinates $\left(x_{0}^{\prime}, y_{0}^{\prime}\right)$ in the slant 


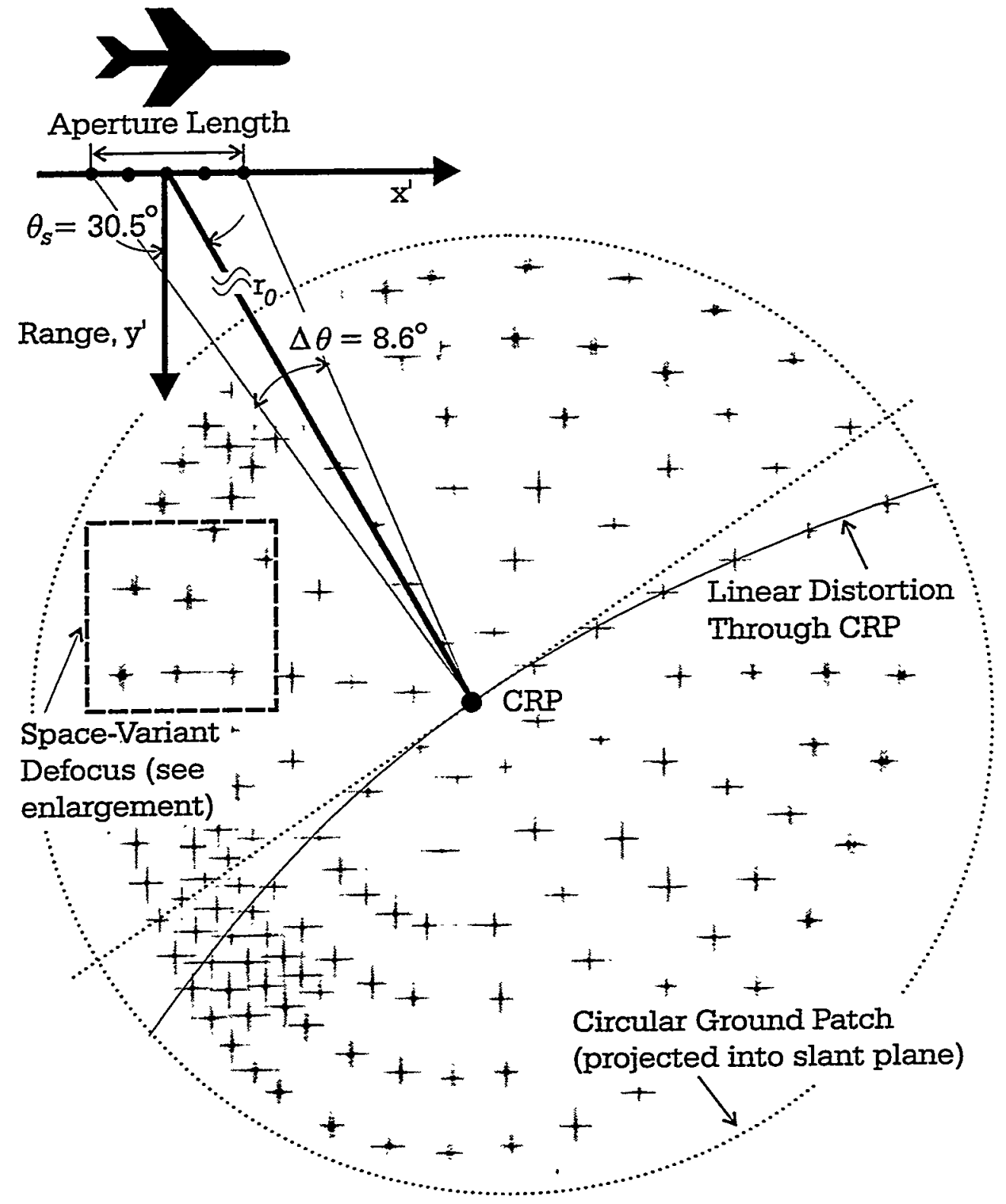

Figure 6.1: L-Band Squint-Mode Collection Geometry with Associated SYNTARG Target Patch (No Wavefront Curvature Correction)

plane are sufficient for correcting defocus in the slant plane image, without regard to how an imaged object is projected from the ground into the slant plane.

Figure 6.2 presents an expanded view of the defocused area bounded by the rectangular dashed-line box within Figure 6.1. In Figure 6.2 (a), this area is shown 


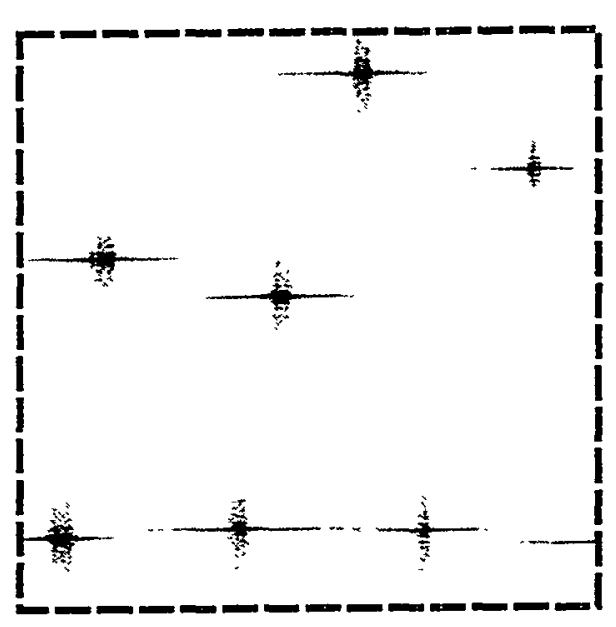

(a)

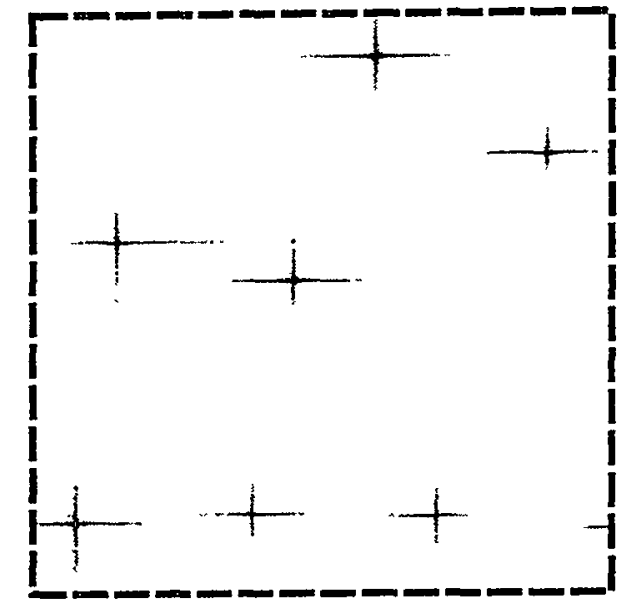

(b)

Figure 6.2: Enlarged View: Squint-Mode Point Targets Before and After Refocus $\left(\theta_{s}=30.5^{\circ}\right)$

prior to space-variant post-filtering, and in view (b), the filter has been applied and the patch refocused. These point targets differ from those shown previously in that the sidelobes have not been reduced through Taylor aperture weighting. The phase history trimming is performed with respect to the standard $\left(x^{\prime}, y^{\prime}\right)$ Cartesian coordinate system and consequently, IPR sidelobes are oriented strictly horizontally and vertically even for this squinted collection scenario. Casual observation of the point targets before and after filtering helps to reassure oneself, at least in a qualitative sense, that the equations and method used to refocus the targets are correct, even for this complicated squint-mode scenario. Figure 6.3 demonstrates in a more quantitative manner, the quality of refocus for a squint-mode point target. The impulse frequency response (IPR) plots of Figure 6.3 (c) represent a point target near the edge of the scene before and after refocusing, as illustrated in Figure 6.3 (a) and (b), respectively. Of particular interest are the elevated sidelobes appearing to the left of the mainlobe in Figure 6.3 (c). While not clearly seen in the refocused, magnitudedetected image of part (b), the IPR plot of (c) reveals this anomaly, which is due to the uncompensated higher-order phase errors present in the phase history due to wavefront curvature. Recall that the Taylor series expansion was carried out only 
through the second order terms. At that time, it was stated (without supporting evidence or mathematical analysis) that the remaining, higher-order phase terms were dominated by the second order terms. Thus, these higher-order terms were thought to have a minimal impact on the residual defocus after space-variant post-filtering. The plot of Figure 6.3 (c) shows that there is some residual higher-order phase error present in the refocused target; however, even for this severe case of L-Band targets located near the scene edge, the effect of this residual phase error is imperceptible to the observer of the imagery, as seen in part (b). Should it ever be necessary to compensate the third-order (or higher) phase error, this would require the expansion of the Taylor series to the third-order (or higher) terms, and the consequent integration of this additional phase correction factor into the space-variant post-filter. It remains to be seen whether these higher-order terms would also be one-dimensional in cross-range. This research activity is suggested in the Future Work section of Chapter 7.

The broadside, L-Band SYNTARG point target scenario has been revisited in terms of quality of refocus, and the results are shown in Figure 6.4. The imaging parameters for this scenario are the same as those given in Table 5.1. In this case, a 40-dB Taylor window has been applied to the Fourier data for sidelobe reduction. Again, while higher-order residual phase errors are evident in the refocused point target, as shown in the IPR plot of Figure 6.4 (c), these are not noticeable in the magnitude-detected image of the refocused target shown in part (b). In fact, given the amount of defocus present in Figure 6.4 (a), the degree of correction shown in part (b) is quite remarkable.

The computationally efficient application of the space-variant post-filter requires that the filter function be varied only as rapidly as necessary to maintain the residual defocus at an acceptable level. This is accomplished by sufficiently overlapping adjacent filter operations to ensure that the extent of the spatial blur is compensated, and by limiting the spatial displacement between adjacent filter applications as to constrain the residual defocus present after filtering. In essence, this concept is that 


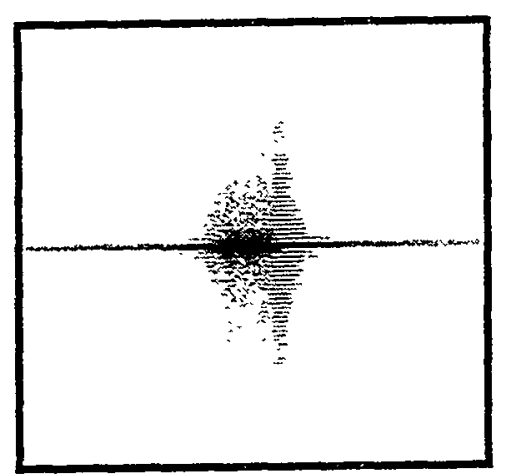

(a)

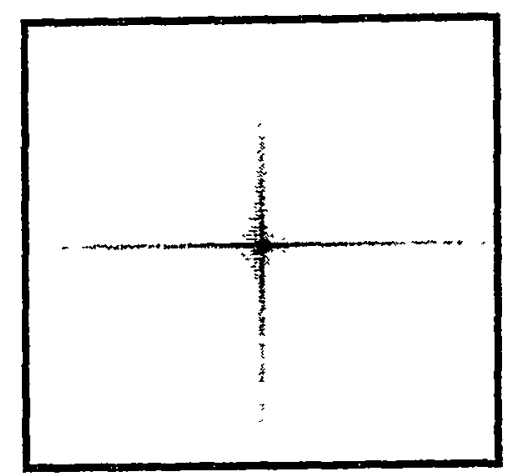

(b)

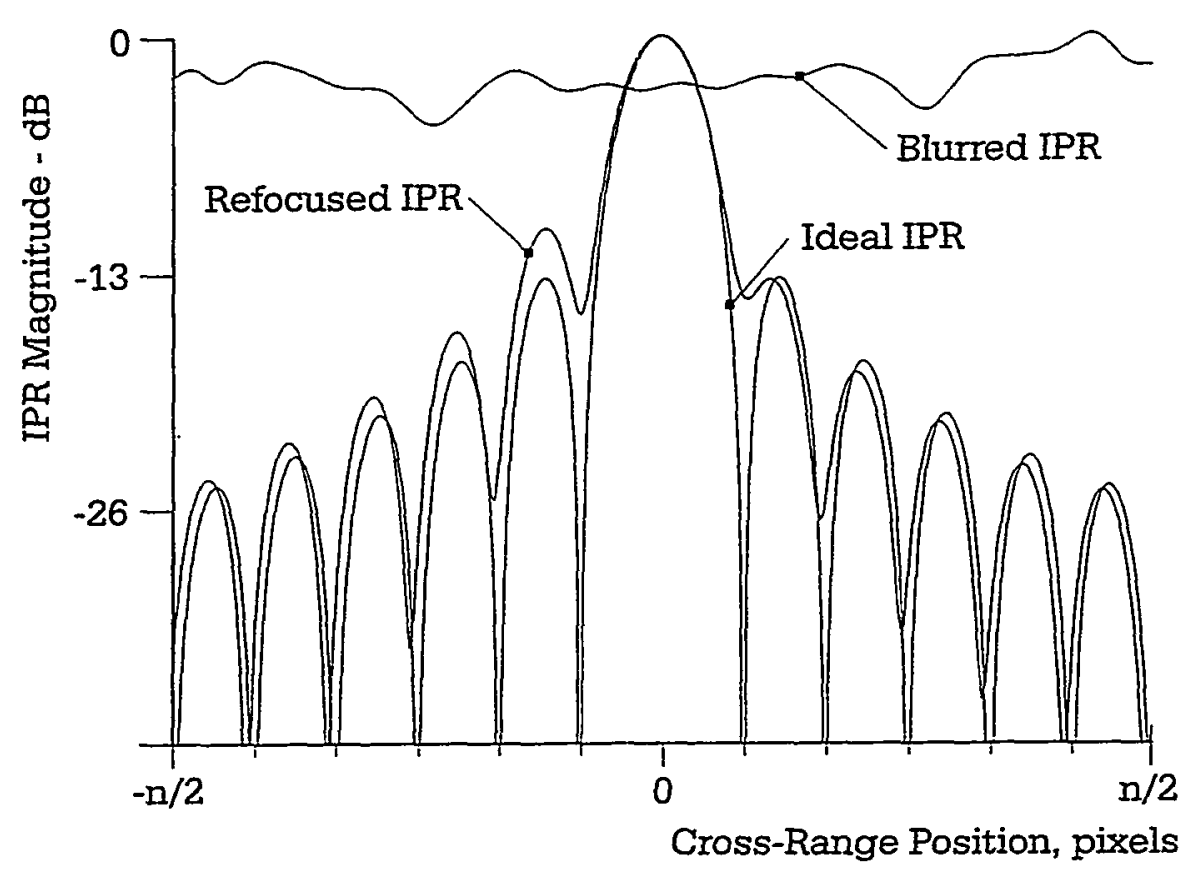

(c)

Figure 6.3: Refocused L-Band Point Target with Associated IPR Plot (Squinted Collection Geometry, $\theta_{s}=30.5^{\circ}$ )

of a space-variant, spatial-domain convolution filter that is adjusted and applied in discrete steps across a continuum of image points. The proper selection of the filter overlap $c$, displacement $d$, and length $m$ are dictated by Equations (5.30), (5.41) and (5.42) of Chapter 5, respectively, and are repeated here for convenience. The 
minimum amount of overlap $c$, in pixels, is defined such that

$$
c \geq \frac{4\left|\phi_{x_{0}^{\prime}, y_{0}^{\prime}}\left(X_{1}^{\prime}\right)\right| K_{a} o_{x}}{\pi}, \quad \text { (pixels) }
$$

where $\phi_{x_{0}^{\prime}, y_{0}^{\prime}}\left(X_{1}^{\prime}\right)$ is the space-variant quadratic phase error as given by Equation (5.1), (5.4) or (5.5). The particular imaging scenario and amount of approximation that is acceptable dictates which phase error equation for $\phi_{x_{0}^{\prime}, y_{0}^{\prime}}\left(X_{1}^{\prime}\right)$ will apply. Furthermore, $K_{a}$ is the IPR mainlobe broadening factor due to windowing (sidelobe control) and $o_{x}$ is the oversample factor in cross-range. The filter displacement distance $d$, in pixels, is constrained as follows:

$$
d \leq \frac{\pi r_{0} k_{0}}{2 s_{x}\left|x_{0}^{\prime}\right| X_{1}^{\prime 2}}=\frac{\rho_{x}^{2} r_{0} k_{0}}{2 \pi s_{x}\left|x_{0}^{\prime}\right|}, \quad \text { (pixels) }
$$

where $k_{0}$ is the nominal phase history radius, $r_{0}$ is the slant range at broadside, $s_{x}$ is the cross-range scale factor, and $x_{0}^{t}$ is the spatial filter distance from the CRP in cross-range. This constraint on $d$ ensures the residual quadratic phase error upon refocus is limited to $\pi / 4$ radians, and consequently, the image blur will be held to subpixel levels. Finally, the filter length $m$ must be equal to $d+c$. That is,

$$
m=c+d
$$

It has been suggested that with the proper selection of filter overlap, displacement and length parameters, as dictated by the design equations of $(6.6),(6.7)$ and (6.8), there will be no visual discontinuities in the image data upon refocusing. This is demonstrated for a set of closely placed point targets, as shown in Figure 6.5 (a). This figure represents a broadside collection of L-Band point targets whose parameters are given by Table 5.1 , and covers an area of $120 \times 120$ pixels. The filter calculations are based on the discussion of Section 5.5 and Equations (6.6) and (6.7). The overlap is calculated to be $c \geq 40$ pixels and the displacement is found to be $d \leq 98$ pixels. The filter length $m$ is equal to $c+d$ by Equation 6.8, and since $d$ can be less than 98 pixels, $m$ is chosen such that $m=64$ (a power of 2 ), $c=40$, and $d=m-c=24$ pixels. Thus, a refocus filter of (FFT) length 64 , displacement of 24 , and overlap of 
Chapter 6. Performance of the Space-Variant Post-Filter

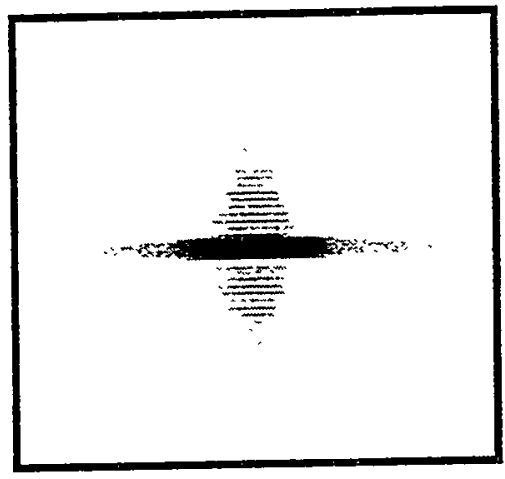

(a)

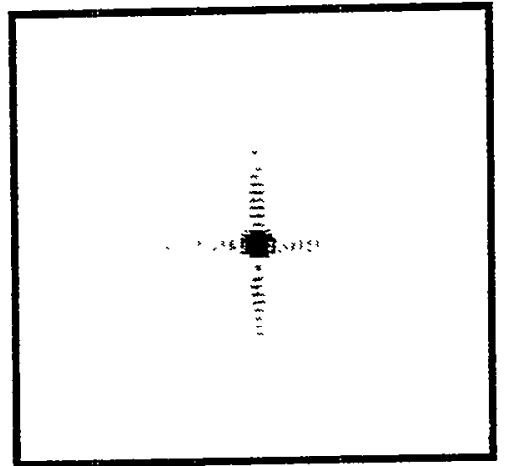

(b)

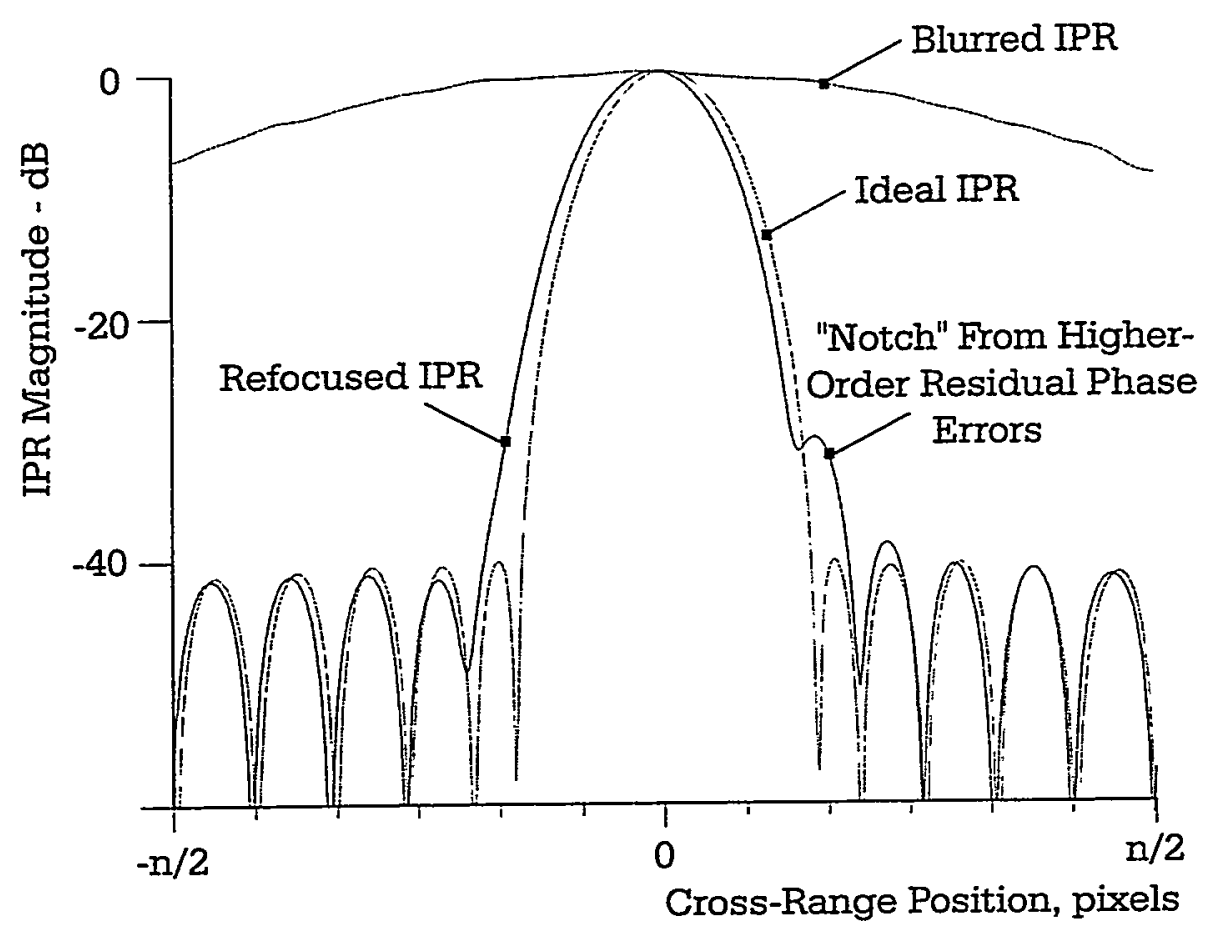

(c)

Figure 6.4: Refocused L-Band Point Target with Associated IPR Plot (Broadside Collection Geometry)

40 (all values in pixels) is applied to the crowded point target set of Figure 6.5 (a), and this filter meets all the specified constraints. In this case, the quadratic phase correction factor is adjusted and reapplied every 24 pixels, as specified by $d$. Thus, 
six discrete filter operations are applied in a sweeping motion across the image data in the cross-range direction, each overlapping the previous by 40 pixels, in order to span the image area which is 120 pixels in cross-range. As is shown in Figure 6.5 (b), the targets are refocused without visible discontinuities. The IPR frequency response plot of Figure 6.5 (c) shows the quality of refocus, and helps to confirm (at least for one example) that the parameter calculations are appropriate. As will be discussed in the Future Work section of Chapter 7, any phase discontinuities present in the data need still be removed before using the image (as one of a pair) for an interferometric estimation of terrain height.

\subsection{Computational Complexity}

\subsubsection{General Complexity}

Many possible approaches exist to evaluate the computational complexity of the space-variant post-filter. One approach is the estimation of the number of complex operations for the algorithm. This approach is consistent with that often used to analyze the running time of image formation algorithms. Consequently, via the complex operations count, the additional computational burden of the space-variant postfilter can be analyzed with respect to the standard PFA algorithm. Furthermore, the performance of PFA in conjunction with space-variant post-filtering (PFA/SVPF) can be compared to other competitive image formation algorithms which compensate wavefront curvature.

A complex operation is one radix-2 FFT butterfly, and a single radix-2 FFT butterfly consists of one complex multiply and two complex additions. Thus, the butterfly equates to four floating point multiplies and six floating point additions for a total of ten floating point (real) operations. Assuming an equal computational cost for both multiplies and additions, it is reasonable to use a 10-to-1 conversion from 
Chapter 6. Performance of the Space-Variant Post-Filter

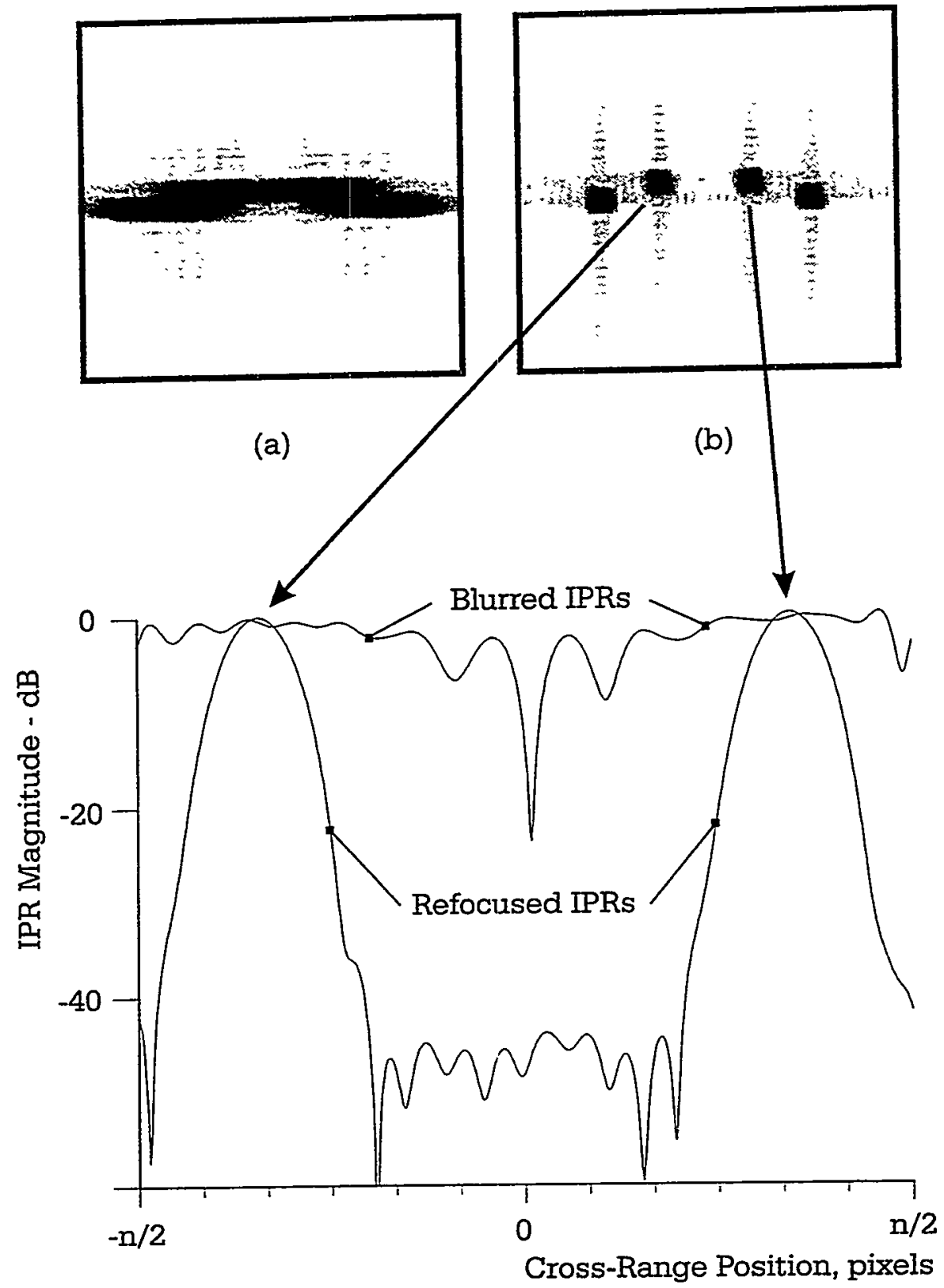

(c)

Figure 6.5: Resolvability of Crowded L-Band Targets (Broadside Collection Geometry)

complex to floating point operations (flops) for FFTs as well as at all other stages of evaluation [12, pp. 479]. Thus, it is a simple matter to convert between complex and floating point operations, if necessary. The complex operation count $C_{f f t}$ for a 
radix-2 FFT of size $m$ is discussed in [90] and found to be

$$
C_{f f t}=\left(\frac{m}{2}\right) \log _{2} m
$$

With regard specifically to the process of space-variant post-filtering, each filter kernel of length $m$, where $m$ is typically a power of two, is multiplied, point by point, by the Fourier transform of the $m$ image data pixels centered at the image location $\left(x_{0}^{\prime}, y_{0}^{\prime}\right)$. The filtered data are then inverse Fourier Transformed into refocused imagedomain data. In terms of complex operations, the forward/inverse Fourier transform operation in conjunction with the with conjugate-multiply filter operation done in between yields $m \log _{2} m+m$ operations according to Equation (6.9). Given a rectangular image of $n_{x} \times n_{y}$ pixels in the cross-range and range dimension, respectively, as well as a filter overlap of $c$ pixels and filter displacement of $d=m-c$ pixels, the overall complex operations count $C_{p}$ for space-variant post-filtering is found to be

$$
C_{p}=\frac{m n_{x} n_{y}}{m-c}\left(\log _{2} m+1\right)
$$

In terms of complex operations per pixel $C_{p / p i x}$, the overall operations count is divided by the number of image pixels:

$$
C_{p / p i x}=\frac{m}{m-c}\left(\log _{2} m+1\right) .
$$

As previously stated, the floating point operations count per pixel, $C_{f / p i x}$, is ten times that of the complex operations count:

$$
\begin{aligned}
C_{f / p i x} & =10 \times C_{p / p i x} \\
& =\frac{10 m}{m-c}\left(\log _{2} m+1\right) .
\end{aligned}
$$

Recall that the filter overlap $c$ has a minimum value dictated by Equation (6.6) and the displacement $d$ is constrained to the maximum value specified by Equation (6.7). Consequently, according to Equation (6.8), the filter length $m$ is the sum of $c$ and $d$. However, the overlap $c$ can be further increased, and/or the displacement $d$ further reduced, while still meeting the specified filter criteria, as long as $(m-c=d) \geq 1$. 
The floating point operations count $C_{f / p i x}$ of Equation (6.12) is at its worst when the displacement $d$ is minimal; that is, when $d=m-c=1$. In this case, the upper bound on computational complexity, big-oh or $O()[84, \mathrm{pp} .23-41]$, is found to be

$$
O\left(C_{f / p i x}\right)=m \log _{2} m
$$

where $m$ is the length of the space-variant post-filter, for some fixed-dimension image of $n_{x} \times n_{y}$ pixels. This complexity is equal to that of the image-wide (and tall), two-dimensional Fourier transform which serves as the basis of the polar-format algorithm. Thus, one may already take comfort in knowing that the space-variant post-filtering step surely will not dominate the PFA computation time, particularly upon considering the additional, costly polar-to-rectangular reformatting step required of PFA. Indeed, as will soon be demonstrated, the post-filtering step increases the overall computational burden of PFA by as little as thirty percent.

As described by Equation (6.12) the floating point operations count $C_{f / p i x}$ varies as a function of the values $m$ and $c$. Consequently, a graphical representation of the operations count consists of a family of curves, each curve representing some fixed overlap value of $c$ and varying over some range of $m$. This family of curves, representing the floating point operations count (per pixel) for a reasonable range of $c$ and $m$, is given in Figure 6.6. The computational curves are nested as a function of $c$, as observed in Figure 6.6. Thus, in order to reduce computation time, it is generally desirable to minimize the filter overlap $\mathrm{c}$ for a given imaging scenario. The effect of increasing the overlap $c$ is to move to a higher computational operations curve, while having no beneficial effect on the quality of refocus of the post-filtered image. In situations where the filter length $\mathrm{m}$ must be increased (to fit a power of two), and the displacement $d$ has been extended to its maximum, then $c$ may have to be increased, such that $c+d=m$, with no detrimental effect on quality of refocus but at an additional computational cost.

Once having been chosen, the values of $c, d$ and $m$ may remain fixed as the postfilter is moved across the image, assuming these values are calculated from Equations 


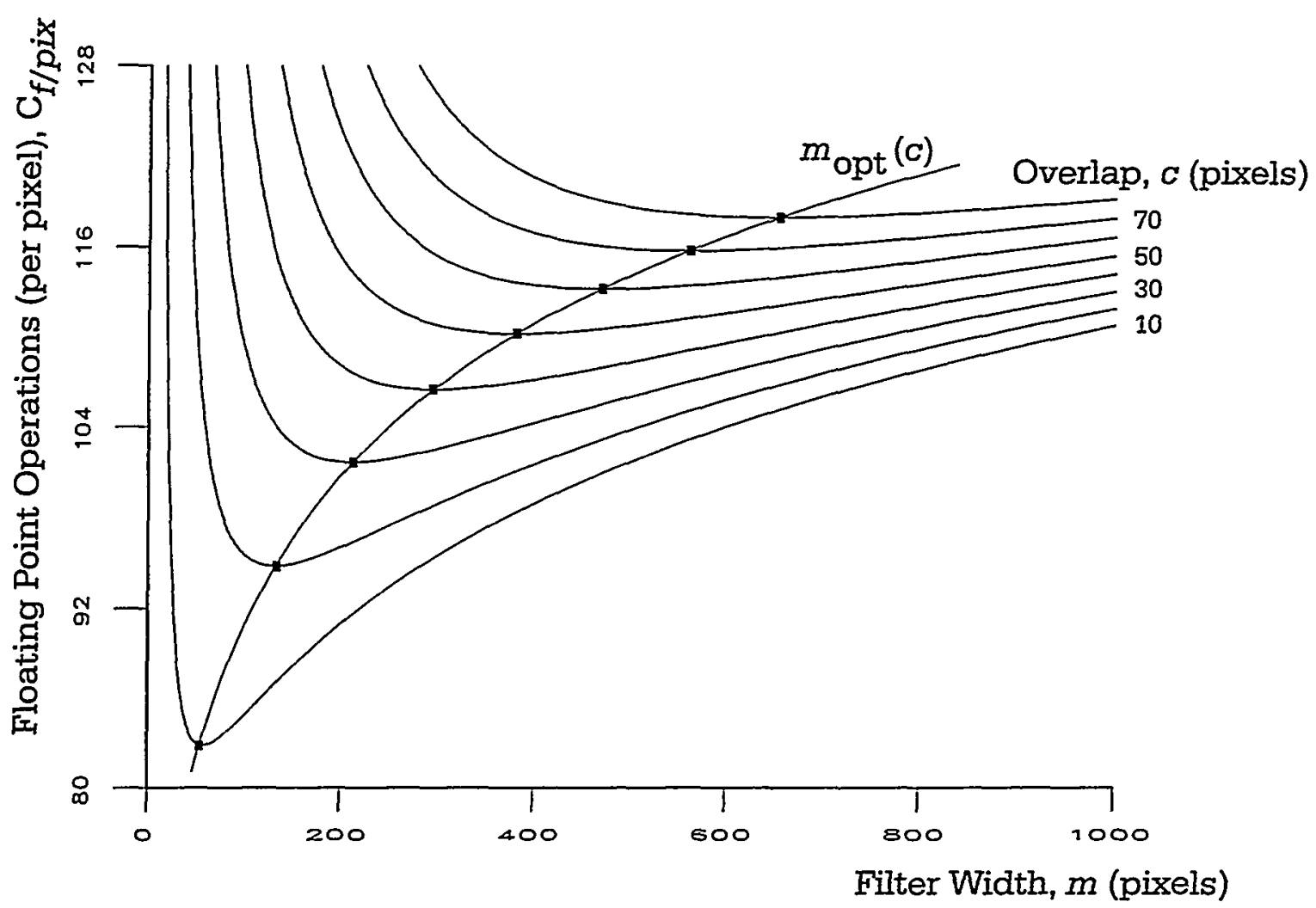

Figure 6.6: Post-Filter Operations Count as a Function of Filter Width and Overlap

(6.6) and (6.7) and are based on the worst case quadratic phase error $\phi_{2 t}$ for the entire image, which occurs at points $\left(x_{0}^{\prime}, y_{0}^{\prime}\right)$ along the Cartesian axes at the far edges of the scene (for broadside collections). In this case, the overall computational burden can be calculated simply by multiplying the appropriate flops/pixel value calculated from Equation (6.12) (or Figure 6.6) by the total number of pixels in the image. The overall computational burden can be reduced by varying $c, d$ and $m$ as a function of filter position $\left(x_{0}^{\prime}, y_{0}^{\prime}\right)$, but this computational analysis, based on this scheme of space-variant overlap adjustment, is beyond the scope of this dissertation. However, even for a fixed filter overlap $c$, an optimal, fixed filter length $m$ can be chosen so as to reduce the overall computational cost of space-variant post-filtering, as shown next. 


\subsubsection{Adjusting Filter Parameters to Reduce Computational Cost}

For each overlap value $c$, an optimal filter length $m_{\text {opt }}$ exists which minimizes the computational cost for that $c$. This optimal filter length is given by $m_{o p t}(c)$ and is derived as follows. The filter length calculation of Equation (6.12) is a function of $m$ and $c$ and is shown to be

$$
\begin{aligned}
C_{f / p i x} & =C_{f / p i x}(m, c) \\
& =\frac{10 m}{m-c}\left(\log _{2} m+1\right) .
\end{aligned}
$$

The minimum value of $C_{f / p i x}(m, c)$ is found by setting its partial derivative, with respect to $m$, equal to zero. This yields the optimal filter overlap $c$ for some given filter length $m$, or $c_{\text {opt }}(m)$, as follows: From Equation (6.15),

$$
\begin{aligned}
\frac{\partial C_{f / p i x}(m, c)}{\partial m} & =\frac{\partial \frac{10 m}{m-c}\left(\log _{2} m+1\right)}{\partial m} \\
& =\frac{10}{m-c}\left(\frac{\ln m}{\ln 2}+1\right)-\frac{10 m}{(m-c)^{2}}\left(\frac{\ln m}{\ln 2}+1\right)+\frac{10}{(m-c) \ln 2} .
\end{aligned}
$$

Setting the RHS of Equation (6.17) to zero and solving for $c$ yields the following:

$$
\frac{10}{m-c}\left(\frac{\ln m}{\ln 2}+1\right)-\frac{10 m}{(m-c)^{2}}\left(\frac{\ln m}{\ln 2}+1\right)+\frac{10}{(m-c) \ln 2}=0
$$

Reducing this yields

$$
\begin{aligned}
& (m-c)\left(\frac{\ln m}{\ln 2}+1\right)-m\left(\frac{\ln m}{\ln 2}+1\right)+\frac{m-c}{\ln 2}=0 . \\
& -c\left(\frac{\ln m}{\ln 2}+1\right)+\frac{m}{\ln 2}-\frac{c}{\ln 2}=0 .
\end{aligned}
$$

Solving Equation (6.20) for $c$ yields

$$
-c\left(\frac{\ln m}{\ln 2}+1+\frac{1}{\ln 2}\right)=-\frac{m}{\ln 2}
$$


and further reducing this relation results in

$$
\left(\frac{\ln m+\ln 2+1}{m}\right)=\frac{1}{c} \text {. }
$$

Furthermore,

$$
c=\left(\frac{m}{\ln m+\ln 2+1}\right)=\left(\frac{m}{\ln m+1.6931}\right) .
$$

Finally, because this development is based on the partial derivative expression of Equation (6.17), then

$$
c_{\text {opt }}(m)=\left(\frac{m}{\ln m+1.6931}\right)
$$

is the optimal filter overlap $c$ for some given filter length $m$. However, for maximum computational efficiency, it is desirable to use the minimum value for the overlap $c$ as calculated from Equation (6.6), and to adjust $m$ to achieve the optimal (minimized) computational burden while obeying the constraint on displacement $d$ as specified by Equation (6.7). Consequently, it is necessary to derive the optimal filter length $m$ for a given overlap $c$; that is, to derive $m_{\text {opt }}(c)$. This requires the inversion of Equation (6.24), such that $m_{\text {opt }}(c)=c_{\text {opt }}{ }^{-1}(m)$. Careful inspection of Equation (6.24) indicates that the mathematical inversion required to represent $m$ as a function of $c$ is not straightforward. As an alternative to direct mathematical inversion, one may fit a curve to the data, whereby the calculated value $c_{\text {opt }}(m)$ is considered the independent value and subsequently, a function is derived (fitted) to determine $m$. This curve fits the computational minima of each function of $m$ (for a given overlap c) and consequently, describes the computationally optimal filter length $m_{\text {opt }}$ for a given overlap $c$, or $m_{\text {opt }}(c)$, as desired. The fitted curve for $m_{o p t}(c)$ is described by the polynomial

$$
m_{\text {opt }}(c)=1.265 \times 10^{-2} x^{2}+7.43 x-21.393
$$

The filter length $m$ which yields the optimal computation time for a given overlap $c$ is determined by $m_{\text {opt }}(c)$ and is plotted in Figure 6.6. For a given imaging scenario, 
it is desirable to adjust the length of $m$ towards the optimal described by $m_{\text {opt }}(c)$, while keeping $c$ constant (and on the lowest allowable computational curve, as defined by Equation (6.6)), in an effort to minimize the overall operations count. All the while, the displacement value $d$ is equal to $m-c$ and constrained from above by Equation (6.7), while minimum allowable value for $d$ is one. That is, for a any fixed value of $c, m$ may be adjusted towards the optimal value $m_{\text {opt }}(c)$, providing $(m-c) \geq 1$ and $d=(m-c)$ is less than the maximum prescribed by Equation (6.7). Additionally, depending on the type of FFT implemented, there may be a constraint on the allowable values for the filter length $m$; for example, a power of two when a radix-2 FFT is incorporated. In such a case, the values of $c$ and $d$ are to be adjusted within their allowable constraints to achieve the best possible computational efficiency, for a value of $m$ which meets the given FFT size constraints.

\subsubsection{Additional Computational Burden of Post-Filtering to Polar-Formatting}

At this point, it is timely to discuss the additional computational burden imposed upon the polar-format algorithm by the space-variant post-filtering process. To this end, the operations count $C_{f / p i x}$ of Equation (6.12) (and associated graph of Figure 6.6) will be further examined. Equation (6.25) defines the optimal filter length $m_{\text {opt }}(c)$ yielding the minimum operations count for some overlap $c$. Thus, the optimized floating operations count $C_{o p t_{f} / p i x}$ for this overlap $c$ is

$$
C_{\text {opt } f / p i x}=C_{f / p i x}\left(m_{o p t}(c), c\right)
$$

and the associated optimal filter displacement $d_{\text {opt }}(c)$ is

$$
d_{o p t}(c)=m_{o p t}(c)-c
$$

From Equations (6.12), (6.25) and (6.26), the optimal floating point operations count for a filter with an overlap $c$ of 10 pixels is $C_{o p t_{f} / p i x}=C_{f / p i x}\left(m_{\text {opt }}(10), 10\right)=$ 
$C_{f / p i x}(54.17,10)=82.9$ flops $/$ pixel. Similarly, for a filter of overlap of $c=80$ pixels, $C_{o p t_{f} / p i x}=C_{f / p i x}\left(m_{o p t}(80), 10\right)=C_{f / p i x}(653.65,80)=118.0$ flops $/$ pixel. Thus, for the range of overlaps and filter lengths representative of typical imaging scenarios, as shown in Figure 6.6, the floating point operations count ranges from about 83 to 118 flops/pixel, specifically for the post-filtering process. Next, this operations count must be compared to that of the polar-formatting algorithm in order to determine the relative computational burden of post-filtering with respect to polar-formatting. A thorough analysis of the floating point operations count for the polar-format algorithm is presented in Carrara's textbook [12, pp. 479-482]. Carrara calculates the operations count to be approximately 280 floating point (real) operations per pixel, for a full-featured PFA implementation. Consequently, polar-formatting in conjunction with the new space-variant post-filter yields an overall operations count ranging from 363 to 398 flops/pixel, resulting in an additional computational burden of 30 to 42 percent over that of the 280 flop, polar-format algorithm, alone. This increase in computational burden applies only to broadside collections. The effect of squint-mode collections, in terms of additional computational burden over those of broadside, is discussed in Section 6.3. The calculations of this section predict an increase in overall computational burden as small as thirty percent due to spacevariant post-filtering. In Section 6.4, these calculations will be found to agree with actual computer simulations.

\subsection{Effect of Squint on the Computational Burden of Space-Variant Post-Filtering}

It is well known that squint negatively impacts the computational burden of certain image formation algorithms which inherently compensate wavefront curvature effects. For example, an important attribute of range migration algorithm (RMA) is its ability to completely compensate migration through range cells, including the geometric 
distortion and higher-order phase errors (leading to space-variant defocus) which are inherent to polar-formatting. However, in squinted scenarios, RMA may become very computationally burdensome. This drawback occurs because RMA must operate on azimuth chirped data resulting from motion compensation to a line, and consequently, in highly squinted scenarios, RMA requires high along-track sampling rates at the front-end of the RMA processor [12, pp. 438]. Methods to alleviate this problem are discussed in $[16,18]$, the latter reference introducing frequency domain replication and downsampling ( $\mathrm{FReD}$ ), a modified RMA method that uses replicated spectrum to mitigate the aliasing effects that occur when using lower along-track sampling frequencies.

In polar-formatting, data is motion compensated to a point and migration through range cells (MTRC) is compensated via sampling on a polar grid and subsequent polar-to-rectangular reformatting. Consequently, there is no azimuth chirp in squintmode scenarios and therefore, no additional computational burden is imposed for squinted collections since there is no increased upsampling. However, unlike RMA and FReD, polar-formatting is based on the planar wavefront assumption of the tomographic paradigm, such that wavefront curvature defocus effects are not mitigated except through space-variant post-filtering. Unfortunately, as will be shown next, the computational burden of space-variant post-filtering increases with increasing squint. Thus, as with RMA, full mitigation of wavefront curvature effects via the polar-formatting algorithm means incurring an additional computational burden (in the post-filtering step) when dealing with squint-mode data. However, in polarformatting, this computational burden is the result of a different phenomenon. Thus, as opposed to RMA, the additional computational burden associated with processing squint-mode data is not particularly severe for polar-formatting when used in conjunction with space-variant post-filtering. The additional computational burden for PFA/SVPF is due an increase in the quadratic phase error induced by wavefront curvature. This is much less severe than the computational burden of upsampling azimuth chirped data, as required by RMA in squinted scenarios. 
A consequence of the planar wavefront assumption of tomography is an increase in the maximum quadratic phase error in squint-mode scenarios. Thus, for a polarformatted scene covering some range of spatial locations $\left(x_{0}^{\prime}, y_{0}^{\prime}\right)$ over a circle of illumination given by $x^{\prime 2}+y^{\prime 2}=\left(D_{p} / 2\right)^{2}$ where $\left(-x^{\prime} \leq x_{0}^{\prime} \leq x^{\prime}\right),\left(-y^{\prime} \leq y_{0}^{\prime} \leq\right.$ $y^{\prime}$ ) and $D_{p}$ is the scene diameter, the overall maximum observed quadratic phase error will be larger (in magnitude) than that seen in a broadside scenario, when all other parameters remain equal. At some specific scene locations, the squintmode quadratic phase error is larger than would be found in a broadside scenario, and at other locations, smaller. However, the overall maximum quadratic phase error is larger in squint-mode. Both the overlap $c$ and displacement $d$ of the spacevariant post-filter are influenced by the amount of quadratic phase error present. In situations where the filter overlap and displacement are fixed in value regardless of scene location, and when these values are based on the maximum quadratic phase error present, then the computational performance of the filter suffers.

The effect of squint on the computational performance of the space-variant postfilter will now be explored. This analysis begins by revisiting the quadratic phase error equation for wavefront curvature. The approximate form of Equation (4.134), based the small patch assumption, is sufficiently accurate for this discussion since a reasonable estimate of the squint-mode computational burden is all that is necessary. This form of the equation is straightforward and allows for an intuitive analysis of the squint-mode computational burden. While based on a mathematical approximation as opposed to an exact expression, additional precision would not lend to a clearer understanding of the computational burden due to squint. Furthermore, by their nature, computer algorithms contribute additional uncertainly in computational burden depending on their specific implementation. Thus, it is hoped that the decision to use this approximation has been justified to the reader.

The generalized (squint-mode) quadratic phase error $\phi_{2 t}$ for the small patch approximation is given by Equation (4.134) in Chapter 4, Section 4.4.3, and is reintro- 
duced here:

$$
\begin{aligned}
f_{\widehat{x}^{2^{\prime}}} X^{X^{\prime 2}} & =\phi_{2 t} \\
& \approx \frac{1}{2}\left\{\frac{x_{0}^{\prime 2}-y_{0}^{\prime 2}}{r_{0} k_{0}}-\tan \theta_{s}\left[\frac{2 x_{0}^{\prime} y_{0}^{\prime}}{r_{0} k_{0}}\right]-\tan ^{2} \theta_{s}\left[\frac{x_{0}^{\prime 2} y_{0}^{\prime}}{r_{0}^{2} k_{0}}\right]\right\} X^{\prime 2}, \quad x_{0}^{\prime}, y_{0}^{\prime} \ll r_{0} .
\end{aligned}
$$

This equation is separated into addends which represent their reliance on squint. The first addend is independent of squint and is the term upon which the post-filter overlap $c$ and displacement $d$ are based (for the broadside imaging case). The second and third addends are functions of the squint angle $\theta_{s}$. The first squint addend (with the $\tan \theta_{s}$ coefficient) contains an $x_{0}^{\prime} / r_{0}$ term, while the second squint addend (with the $\tan ^{2} \theta_{s}$ coefficient) contains an $x_{0}^{\prime 2} / r_{0}^{2}$ term. Since this is a small patch approximation where $x_{0}^{\prime}, y_{0}^{\prime} \ll r_{0}$, then $x_{0}^{\prime 2} / r_{0}^{2} \ll x_{0}^{\prime} / r_{0}$. Thus, the first squint addend dominates the second, and Equation (6.28) can be further approximated:

$$
\phi_{2 t} \approx \frac{1}{2}\left\{\frac{x_{0}^{\prime 2}-y_{0}^{\prime 2}}{r_{0} k_{0}}-\tan \theta_{s}\left[\frac{2 x_{0}^{\prime} y_{0}^{\prime}}{r_{0} k_{0}}\right]\right\} X^{\prime 2}, \quad x_{0}^{\prime}, y_{0}^{\prime} \ll r_{0} .
$$

From Equation (6.29), the squint term is maximal and the non-squint term zero for points $\left(x_{0}^{\prime}, y_{0}^{\prime}\right)$ along the diagonals given by $y^{\prime}=\left|x^{\prime}\right|$. Conversely, along the Cartesian axes defined by $x^{\prime}$ and $y^{\prime}$, the non-squint term is maximal and the squint term is zero. In order to derive the additional computational burden of squint mode processing on the space-variant post-filter, the quadratic phase error equation at broadside must be revisited. Consider Equation (6.29), where $\theta_{s}=0$. This is the broadside-specific case of the generalized phase error equation, denoted by $\phi_{2 t_{\text {broad }}}$. In this case, $\tan \theta_{s}=\tan (0)=0$, and Equation (6.29) reduces to

$$
\phi_{2 t_{\text {broad }}} \approx \frac{1}{2}\left\{\frac{x_{0}^{\prime 2}-y_{0}^{\prime 2}}{r_{0} k_{0}}\right\} X^{\prime 2}, \quad x_{0}^{\prime}, y_{0}^{\prime} \ll r_{0}
$$

which is that of the broadside imaging case, first given by Equation (4.135).

For a polar-formatted scene covering some range of spatial locations $\left(x_{0}^{\prime}, y_{0}^{\prime}\right)$ over a circle of illumination given by $x^{\prime 2}+y^{\prime 2}=\left(D_{p} / 2\right)^{2}$ where $\left(-x^{\prime} \leq x_{0}^{\prime} \leq x^{\prime}\right),\left(-y^{\prime} \leq\right.$ 
$\left.y_{0}^{\prime} \leq y^{\prime}\right)$ and $D_{p}$ is the scene diameter, then

$$
\phi_{2 t_{\max }} \approx \max \left(\frac{1}{2}\left\{\frac{x_{0}^{\prime 2}-y_{0}^{\prime 2}}{r_{0} k_{0}}-\tan \theta_{s}\left[\frac{2 x_{0}^{\prime} y_{0}^{\prime}}{r_{0} k_{0}}\right]\right\} X^{\prime 2}\right), \quad x_{0}^{\prime}, y_{0}^{\prime} \ll r_{0} .
$$

Furthermore, by Equations (6.30) and (6.31),

$$
\phi_{2 t_{\text {max }}} \leq \begin{cases}2 \max \left(\phi_{2 t_{\text {broad }}}\right) & \left|\theta_{s}\right| \leq 45^{\circ}, \\ 2 \tan \theta_{s} \max \left(\phi_{2 t_{\text {broad }}}\right) & 45^{\circ}<\left|\theta_{s}\right|<90^{\circ} .\end{cases}
$$

That is, the maximum quadratic phase error at the signal edge for squinted scenarios $\phi_{2 t_{\max }}$ is at most twice the maximum quadratic phase error found for the same scenario imaged at broadside, given squint angles between \pm 45 degrees. When the squint is between $45^{\circ}$ and $90^{\circ}$ (or between $-45^{\circ}$ and $-90^{\circ}$ ) the maximum quadratic phase error is at most $2 \tan \left(\theta_{s}\right)$ times the maximum phase error when imaged at broadside. Thankfully, while the maximum squint-mode phase error may be twice that (or more) of the equivalent broadside collection, this does not yield a post-filtering computational burden that is twice that of the broadside scenario. In fact, the overall increase in computational burden for the complete polar-formatting process that includes post-filtering may be as little as $5.5 \%$, when considering a squint-mode scenario as opposed to broadside. This increase in burden for the squint-mode scenario will be discussed now.

The post-filter length, overlap and displacement at a spatial position $\left(x_{0}^{\prime} ; y_{0}^{\prime}\right)$ are based on the quadratic phase error present (at the signal edge) $\phi_{2 t\left(x_{0}^{\prime}, y_{0}^{\prime}\right)}\left(X^{\prime}\right)$ at that point in the scene for a given imaging scenario. The basis for calculating the filter displacement $d$ is a relation incorporating the rate of change of the quadratic phase error, or $\dot{\phi}_{\left(x_{0}^{\prime}\right)}\left(X_{1}^{\prime}\right)$, as given by Equation (5.35). Assuming the squint-mode scenario yields a phase error that is (at most) double the broadside phase error found at the point $\left(x_{0}^{\prime}, y_{0}^{\prime}\right)$ (which is valid for squint a angle $\theta_{s} \leq 45^{\circ}$ ), then according to the mathematical development of Equations (5.31)-(5.41), the maximum allowable displacement $d$ at that point is reduced by a factor of two. When the squint angle is even larger, the maximum allowable displacement $d$ varies inversely with respect to 
$2 \tan \left(\theta_{s}\right)$. Similarly, the development of Equations (5.29)-(5.30) reveal that doubling the phase error (for the squint case compared to the broadside, where $\theta_{s} \leq 45^{\circ}$ ) at some spatial position $\left(x_{0}^{\prime}, y_{0}^{\prime}\right)$ requires a doubling of the minimum allowable overlap $c$ between adjacent filter applications at $\left(x_{0}^{\prime}, y_{0}^{\prime}\right)$. For squint angles $\theta_{s}$ greater than $45^{\circ}$, the minimum allowable overlap $c$ between adjacent filter applications increases in proportion to $2 \tan \left(\theta_{s}\right)$. Assume for simplicity (though not maximum efficiency) that the filter length, overlap and displacement are chosen to be fixed values across the entire spatial extent of the image. In this case, the computational operations count, per pixel, is represented by the curves of Figure 6.6 (or Equation (6.12)), and the overall operations count is found by multiplying this value by the total number of image pixels. These curves may represent either a broadside or squint-mode scenario; the maximum value of $d$ reduced and the minimum value of $c$ increased as appropriate for squint-mode scenarios.

Calculating the increased computational burden of the post-filter due to squint can be quite straightforward, given appropriate simplifications. For example, consider a broadside spotlight-mode imaging scenario for a radar of center frequency $f_{0}=5.0 \mathrm{gHz}$, cross-range resolution $\rho_{x}$ of $1.25 \mathrm{~m}$, and patch diameter $D_{p}$ of $2000 \mathrm{~m}$. By equation (6.6), a post-filter overlap of $c=10$ is above the minimum required and thus adequate for proper wavefront curvature correction, given an oversample value $o_{x}=1.92$ and mainlobe broadening factor $K_{a}$ of 1.4. These parameters induce a mild case of wavefront curvature defocus at the edge of the scene, and this example is used as the benchmark scenario for the workstation execution time comparisons of Section 6.4.

For this squint-mode computational burden example, let $c_{b}$ represent the worstcase broadside filter overlap. Therefore, $c_{b}=10$. By equation (6.25), the optimal filter length $m_{\text {opt }}$ is found to be $m_{\text {opt }}(10) \approx 53$. Furthermore, by Equation (6.8), $d_{b}=m_{\text {opt }}-c_{b}=53-10=43$. This value of displacement $\left(d_{b}=43\right)$ is less than the maximum displacement value $d=812$ prescribed by Equation (6.7) for these particular imaging parameters; therefore, the filter constraints are met. Now, the 
additional computational burden due to squint will be discussed. Consider a squintmode scenario where the squint angle $\theta_{s}$ is constrained by the inequality $\left|\theta_{s}\right| \leq 45^{\circ}$. By Equation (6.32), this yields a maximum quadratic phase error for a circular patch that is no more than twice that encountered for the same scene imaged at broadside. Consequently, the maximum allowable displacement $d$ is reduced by a factor of two, and the minimum allowable overlap $c$ is doubled. Thus, for the squint-mode scenario, the minimum filter overlap $c_{s}$ is $2 \times c_{b}=20$ pixels. Assuming the filter length $m$ remains fixed when comparing broadside to squint, then the filter displacement for the squinted scenario $d_{s}$ is found to be $53-20=33$. This is well under the squintmode filter displacement constraint of $d / 2=812 / 2=406$ pixels.

The computational operations counts (in flops/pixel) for the broadside and squint scenarios are given by Equation (6.14). For the broadside scenario, $C_{f / p i x}(m, c)=$ $C_{f / p i x}(53,10) \approx 83 \mathrm{flops} /$ pixel for the space-variant post-filter algorithm. In conjunction with the 280 flops/pixel operations count for the polar-formatting algorithm, the combined computational burden of PFA/SVPF is $280+83=363 \mathrm{flops} /$ pixel. For the squint-mode scenario, $C_{f / p i x}(m, c)=C_{f / p i x}(53,20) \approx 108$ flops/pixel for post-filtering, for a combined PFA/SVPF burden of $280+108=388$ flops/pixel. Consequently, the additional computational burden associated with squint-mode processing is only $(1-388 / 363) \times 100=6.9 \%$ for this example! In a more general sense, as the broadside overlap $c$ varies within the range of $10 \leq c \leq 80$ (depending on frequency, resolution and patch size parameters) and the optimal filter length $m$ is chosen based on this $c$, the additional computational burden for the same scenario imaged at a squint angle $\left|\theta_{s}\right| \leq 45^{\circ}$ ranges from $5.5 \%$ to $6.9 \%$, depending on the initial, broadside-mode value of $c$. Thus, while this additional computational burden for squint-mode with PFA/SVPF is not inconsequential or completely negligible, it does not make a significant impact on overall computation time. This is in stark contrast to the RMA algorithm, whose oversampling requirements in squint mode can create a huge computational burden compared to that of broadside. 


\subsection{Computer Simulation Results}

\subsubsection{Choice of Algorithms}

In this section, several popular image formation algorithms are compared to polarformatting (with and without post-filtering) with regard to the overall running time on a common engineering workstation platform. While not a scientific analysis (compared to the operations count), it does help to verify, through a practical implementation, the theory derived and discussed thus far, with regard to computational burden. It will be shown through these computational simulations that indeed, the burden involved in performing the space-variant image restoration for wavefront curvature is not particularly severe. Furthermore, these benchmarks will demonstrate that polar-formatting in conjunction with space-variant post-filtering is a viable image formation algorithm when wavefront curvature effects are present. In addition to polar-formatting, three other well known image formation algorithms are considered. The seismic migration technique (RMA) is benchmarked as is the newer frequency domain replication and downsampling (FReD) algorithm, a modified version of RMA designed to reduce the along-track upsampling requirements when imaging in squint-mode. Furthermore, the convolution back-projection (CBP) algorithm has been implemented and benchmarked.

While the RMA and FReD algorithms inherently compensate wavefront curvature, the CBP and PFA algorithms are based on the tomographic paradigm and rely on the associated planar wavefront assumption. Consequently, additional measures are required to compensate the wavefront curvature effects in the PFA and CBP implementations. Obviously, space-variant post-filtering is the method of compensation chosen for PFA and is the focus of this dissertation. Similarly, in the recent past, CBP has been suitably modified by other researchers to compensate wavefront curvature. As will be shown next, the computational burden of CBP is severe compared to the other algorithms, even before considering the additional burden as- 
sociated with wavefront curvature correction. This is due to the $O\left(n^{3}\right)$ computational complexity of CBP, resulting from the filtering and projection of $n$ aperture samples across an $n \times n$ image grid. As such, it was deemed unnecessary to benchmark a variation of CBP which compensates wavefront curvature. Furthermore, variations of the aforementioned algorithms, such as the chirp scaling algorithm (CSA) and modified chirp-Z transform (MCZT), both simplifications (via approximation) of RMA, have not been benchmarked. Another class of image formation methods, the orerlapped and tiered subaperture algorithms (OSA and TSA) were originally designed for high-speed image formation when implemented on specialized VLSI hardware. Furthermore, their subaperture approach lends to their inherent compensation for wavefront curvature. While not specifically benchmarked here, the OSA algorithm has been analyzed in detail and found to be computationally competitive with the algorithms studied here, on a flops/pixel basis for single processor systems [91].

The polar-format algorithm being benchmarked is an in-house (Sandia National Laboratories) product whose functionality is examined in Section 1.1 of Chapter 1. The reader is also encouraged to review Section 1.3 of Chapter 1 , which gives many details of the various image formation algorithms, and provides a brief comparison/contrast of these methods with regard to image formation features. Also within Chapter 1 (specifically, Section 1.3), a number of important references have been cited which will assist the reader in the understanding of SAR image formation fundamentals and algorithms.

\subsubsection{Benchmark Results}

The spotlight-mode, SAR imaging parameters for all implemented algorithms are given in Table 6.1. The computer simulation was conducted for a broadside collection only. All algorithms were implemented in the FORTRAN programming language and executed on a Sun Microsystems Ultra ${ }^{\text {tm }}$ workstation running at $200 \mathrm{mHz}$. For the RMA algorithm, the required upsampling ratio is approximately 2:1 for this 


\begin{tabular}{||l|c|c||}
\hline \multicolumn{2}{|c|}{ Radar Parameters for Workstation Timing Experiments } \\
\hline \multicolumn{1}{|c|}{ Parameter } & Symbol & Value \\
\hline \hline Center Frequency & $f_{0}$ & $5.0 \mathrm{gHz}$ \\
Wavelength & $\rho_{x}$ & $0.06 \mathrm{~m}$ \\
Resolution, Cross-range & $\rho_{y}$ & $1.25 \mathrm{~m}$ \\
Resolution, Range & $s_{x}$ & $1.25 \mathrm{~m}$ \\
Scale Factor, Cross-range & $0.65 \mathrm{~m} / \mathrm{pix}$ \\
Oversample Value, Cross-range & $o_{x}\left(o_{x}=\rho_{x} / s_{x}\right)$ & 1.92 \\
Mainlobe Broadening Factor & $K_{a}$ & 1.4 \\
Slant Range at Broadside & $r_{0}$ & $10000 \mathrm{~m}$ \\
Squint Angle & $\theta_{s}$ & $0^{\circ}$ \\
Patch Diameter & $D_{p}$ & $2000 \mathrm{~m}$ \\
Image Dimensions & $n_{x}, n_{y}$ & $4096,4096 \mathrm{pixels}$ \\
\hline
\end{tabular}

Table 6.1: Radar Parameters for Workstation-Based Timing Benchmark

\begin{tabular}{||l|c|c|}
\hline \multicolumn{2}{|c|}{ Workstation Parameters Specific to Space-Variant Post-Filtering } \\
\hline \multicolumn{1}{|c|}{ Parameter } & Symbol & Value \\
\hline \hline Filter Length & $\mathrm{m}$ & 64 pixels \\
Filter Overlap & $\mathrm{c}$ & 10 pixels \\
Filter Displacement & $\mathrm{d},(d=m-c)$ & 54 pixels \\
Image Dimensions & $n_{x}, n_{y}$ & 4096,4096 pixels \\
\hline
\end{tabular}

Table 6.2: SVPF-Specific Parameters for Workstation-Based Timing Benchmark

scenario. The space-variant post-filter parameters, in terms of overlap, length and displacement, are given in Table 6.2. These parameters were calculated from the equations for maximum overlap $c$ and displacement $d$, given by Equations (6.6) and (6.7), respectively, and based on the radar parameters of Table 6.1. The optimal filter length $m_{\text {opt }}(c)$ is based on a value of $c=10$ and therefore, $m_{\text {opt }}(10) \approx 53$ according to Equation (6.25). Since this is a practical implementation, the filter length $m$ has been increased to 64 in order to comply with the power-of-two constraints of the radix-2 FFT being implemented. The value of $c$ remains at $10 \mathrm{in}$ order to stay on the lowest computational curve (see Figure 6.6) and consequently, $d=m-c=54$, which still satisfies the constraint on the maximum filter displacement $d$ as specified by Equation (6.7). 


\begin{tabular}{||l|c|c||}
\hline \multicolumn{1}{|c|}{ Spotlight-Mode Image Formation Benchmark - Broadside Geometry } \\
\hline & $\begin{array}{c}\text { Wavefront Curvature } \\
\text { Defocus Correction? }\end{array}$ & $\begin{array}{c}\text { Execution } \\
\text { Time, seconds }\end{array}$ \\
\hline \hline Polar-Format (PFA) & No & 434 \\
\hline $\begin{array}{l}\text { Polar-Format with Space-Variant } \\
\text { Post-Filtering (PFA/SVPF) }\end{array}$ & $\begin{array}{c}\text { Yes, } \\
\text { Through 2nd Order }\end{array}$ & $\begin{array}{c}569 \\
\text { (PFA + 31\%) }\end{array}$ \\
\hline Range Migration (RMA) & Yes, Full & 1153 \\
\hline $\begin{array}{l}\text { Frequency Domain Replication (FReD) } \\
\text { (Modified RMA) }\end{array}$ & Yes, Full & 713 \\
\hline Convolution Back-Projection (CBP) & No $^{b}$ & 132,000 \\
\hline
\end{tabular}

${ }^{a}$ Fixed filter overlap, length and displacement from one filter application to the next.

${ }^{b}$ Wavefront curvature correction for CBP is discussed in [30,31, 32], but not benchmarked here.

Table 6.3: Execution Times for Five Algorithms in FORTRAN on a Sun Ultra-2 Workstation

The running times of RMA, FReD and CBP algorithms were compared to that of PFA, both with and without the space-variant post-filter. These running times are presented in Table 6.3. The wavefront curvature processing time via space-variant post-filtering is just over two minutes for a $4096 \times 4096$ pixel image on the Ultra workstation. This amounts to about $30 \%$ of the polar-format image formation time. Thus, for this imaging scenario, the entire computing time required for generating an image free of wavefront curvature effects is 569 seconds, when using polar-formatting in conjunction with the new space-variant post-filter. As can be seen from the table, this time compares very favorably to those of RMA and FReD, and is much better than the running time of CBP.

\subsubsection{Observations}

Previously, it was determined that the additional computational burden for postfiltering squint-mode scenarios is minimal relative to that required to refocus a broadside collection of the same scene. Thus, it is sufficient to benchmark the im- 
age formation algorithms for a broadside scenario only. This is reasonable since the $\mathrm{PFA} / \mathrm{SVPF}$ algorithm would remain competitive in squint-mode scenarios, regardless of the increase in computational burden for the other algorithms being analyzed.

This FORTRAN implementation of PFA/SVPF would indicate that it is more computationally efficient than the original version of the seismic migration technique, RMA, and also more efficient than the computationally "improved" FReD algorithm. However, FORTRAN timings are not the final word on algorithm efficiency, especially in situations such as this, where admittedly, algorithms are not necessarily implemented efficiently or tuned for maximum computational benefit. Thus, it is not the intention of this benchmark to prove the "superiority" of polar-formatting to other image formation algorithms. Instead, these timings demonstrate, in an empirical sense, that polar-formatting in conjunction with space-variant post-filtering is a viable candidate for image formation when wavefront curvature effects are present. Furthermore, the benchmark shows that when implemented according to the methodology presented in Chapter 4, and with the specified optimizations, that in fact, a practical implementation of space-variant post-filtering can be expected to perform (more or less) according to the theoretical predictions. The running time of the basic PFA algorithm (without post-filting) is demonstrated to be very similar to that of the RMA algorithm, and this empirical result is consitent with the corresponding flops/pixel operations counts derived by Carrara for these algorithms [12, pp. 481].

Finally, it is important to note that this algorithm comparison is unfair in the sense that it "compares apples to oranges." Specifically, the RMA and FReD algorithms exactly compensate the wavefront curvature defocus effects. In contrast, the space-variant post-filter corrects phase errors only through second-order, leaving higher-order (but usually, visually imperceptible) residuals in the image. Furthermore, the computational efficiency of post-filtering is due, in part, to varying the filter only as often as necessary to reduce the residual second-order phase errors such that the remaining defocus in formed imagery is constrained to subpixel levels. Thus, for most imaging scenarios (except those extreme cases with severe higher- 
order phase components), PFA/SVPF yields an image free of wavefront curvature defocus effects, at least as perceived by the human visual system. In fairness to the other algorithms, this is not a theoretically perfect correction. However, when comparing a perfect correction, as in the case of RMA, to that of an approximation, as with PFA/SVPF, correcting defocus to the point of being "visually imperceptible" meets the design goals of the filter, and thus, is close enough to perfect. However, this design goal is not to be construed as actually being a perfect compensation. Consequently, at first glance, the benchmark seems to indicate the running-time "superiority" of PFA/SVPF as compared to other algorithms, but this must be tempered by taking into account the inherent differences in the corrective capabilities of the algorithms. Similarly, in the case of CBP, while the high computational burden seems to discourage its use, this algorithm has unique capabilities that make it very desirable in some scenarios, despite its burden. For example, the ability of CBP to form images from very long apertures without polar-to-rectangular interpolation, thereby eliminating the associated interpolation errors. 
Chapter 6. Performance of the Space-Variant Post-Filter

This page intentionally left blank 


\section{Chapter 7}

\section{Conclusion}

\subsection{Summary}

The traditional polar-formatting algorithm, PFA, is a robust, efficient and well proven method for spotlight-mode SAR image formation. However, the derivation of this technique relies on the unrealistic assumption of strictly planar wavefronts in the transmitted pulses, as prescribed by the tomographic framework under which it was developed. As the radar system resolution increases, or large scenes are imaged at close range or low center frequencies, the amount of wavefront curvature actually present in the scene differs considerably from that of the assumed planar wavefront. Consequently, for these collections, a significant amount of geometric distortion and space-variant defocus is present. While the geometric distortion is easily removed via a post-warping procedure, the space-variant defocus due to wavefront curvature is more problematic.

This dissertation has introduced a general formulation for wavefront curvature correction in spotlight-mode SAR images formed using the polar-formatting algorithm. This correction is achieved via an efficient, image-domain space-variant filter applied as a post-processing step to PFA. This formulation is general in that it cor- 
rects for wavefront curvature in both squinted and broadside collection modes, with little computational penalty for correcting squint-mode images. Furthermore, except for the most severe cases (which have a significant higher-order residual phase error), correction is successfully performed without constraints on the scene size, radar center frequency, resolution or slant-range.

Prior to post-filtering, space-variant defocus was not compensated but simply reduced to an acceptable level by imposing a restrictive upper limit on the imaged scene size when using polar-formatting. This was an unreasonable constraint that limited the versatility when imaging large scenes; ironic, given that otherwise, PFA lends itself nicely to imaging large scenes due to its simplicity. The post-filter for wavefront curvature correction is precalculated from a theoretical derivation of the wavefront curvature effect, as based on the geometric model for the phase return from a point target. This phase return is broken into components via a Taylor series expansion and the second-order (quadratic) phase error term is considered to be dominant in inducing the defocus. The quadratic phase error varies depending on the spatial location within the imaged scene. Consequently, the post-filter is space-variant to compensate this error as a function of scene location.

The post-filter is made efficient by varying it only as often as necessary to limit the residual blur to an imperceptible level. This residual blur results from the quadratic (and higher-order) phase errors remaining after post-filtering. Furthermore, since the quadratic phase error components are zero in the range direction, the filter need only be one-dimensional in cross-range, even for squinted scenarios, which further lends to its efficiency. Also lending to the efficiency of post-filtering is the linear flight path assumption of the phase model. This assumption yields a closed form phase error estimate which obviates the need for phase compensation at each aperture point along the flight path for every spatial image location. Accordingly, the computational burden is greatly decreased. The linear flight path assumption is restrictive, yet not unrealistic in that it closely models ISAR (inverse SAR, which specifies a fixed antenna imaging rotating objects), valid under the tomographic paradigm. Finally, 
a number of rules have been presented in this dissertation which assist the algorithm designer in further reducing the computational burden via optimal filter sizing and movement, while still meeting quality-of-refocus constraints.

This research has shown that traditional polar-formatting in conjunction with space-variant post-filtering (SVPF) effectively compensates the wavefront curvature defocus in spotlight-mode imagery. Use of the space-variant post-filter effectively eliminates scene size restrictions and constraints on squint angle, resolution and slant-range. Furthermore, SVPF does not require subaperture processing and the associated patchwork reassembly of the image, nor is it iterative or have any dependence on the visual contents of the scene. Consequently, it is an excellent candidate for high performance computing environments via parallelization. In many cases, the computational burden of post-filtering is shown to be as small as thirty percent of the polar-formatting time, and the additional burden from processing squint-mode imagery is typically only six percent. This is in stark contrast to the range migration algorithm (RMA), whose severe along-track upsampling requirements induce a huge computational burden in squint-mode. Modifications to RMA, such as the frequency domain replication (FReD) algorithm, have reduced the squint-mode computational burden by obviating the need to upsample. However, the intent of this dissertation is not to discredit or validate any particular image formation algorithm, but instead, simply to show that PFA in conjunction with space-variant post-filtering is a viable candidate for spotlight-mode image formation when wavefront curvature effects are present. The author feels this has now been satisfactorily demonstrated.

\subsection{Contributions of this Dissertation}

This dissertation makes a number of significant contributions to the concept of spacevariant post-filtering for wavefront curvature correction in polar-formatted spotlightmode SAR. In short, this dissertation provides a comprehensive treatment of space- 
variant post-filtering covering the following topics: general motivation, geometric and phase model construction, phase error derivation and filtering equation development, computer implementation, quality of refocus analysis, operations count and comparative benchmarking against other algorithms. The analysis of space-variant post-filtering was conducted on both broadside and squint-mode scenarios. The individual contributions are detailed as follows.

- Wavefront curvature effects were demonstrated for polar-formatted imagery via numerous pictorial examples. Specifically, geometric distortion and defocus effects were fully demonstrated and the corresponding scene size restrictions were derived for both geometric distortion and space-variant defocus. (Chapter $3)$.

- Geometric distortion was described in terms of a geometric model of the actual curved wavefront, and also presented as a first-order phase error effect via a model of the phase return from a point target. (Chapters 3 and 4).

- The original broadside-only phase return model for wavefront curvature, previously introduced by Jakowatz, et al [7, pp. 355-360], was expanded into a generalized squint-mode model. Consequently, as would be expected, the broadside model and associated equations (representing a squint angle of zero) were shown to be a specific case of the new, generalized squint-mode model. Furthermore, the small-patch approximation proposed by Jakowatz was shown to be yet another special case of the generalized model presented here. (Chapter 4$)$.

- The space-variant post-filtering process, from a computational perspective, was made more efficient by varying the filter only as often as necessary to remain below a maximum acceptable phase error. Efficiency was further enhanced by implementing a one-dimensional refocus filter in cross-range, which was shown to be sufficient for proper compensation. A linear flight path assumption also 
served to reduce the computational burden. It was also demonstrated that the quadratic phase error due to wavefront curvature remains one-dimensional in cross-range even for squint-mode scenarios. (Chapter 5 ).

- Consideration was given to the geometric distortion associated with wavefront curvature, and a refocus (filtering) method proposed that accounted for this distortion without necessitating its correction. In this way, the traditional post-warping step of PFA can be retained. (Chapter 5).

- Quadratic phase errors were mathematically analyzed with respect to their effect on IPR width, and a closed form solution was presented (based on [92, 93]) to predict IPR broadening. (Appendix A).

- Analytical, closed form solutions were derived for overlap $c$, displacement $d$ and length $m$ of the post-filter as a function of $(x, y)$ location in the scene. These parameters were based on rate-of-change calculations for quadratic phase and also the IPR broadening analysis. (Chapter 5).

- An analytical operations count and complexity analysis were done for the postfilter, based on filter overlap, length, and displacement. Suggestions were made (accompanied by the appropriate mathematical analysis) for optimizing the operations count. (Chapter 6).

- Quality of refocus was examined from both a qualitative and quantitative perspective. The SVPF computational burden was calculated to be as little as thirty percent of the polar-format algorithm burden, based on filter overlap, displacement and length (as driven by the original radar parameters and imaging geometry). This burden was shown to be consistent with the empirical running-time workstation benchmarks. (Chapter 6).

- The additional post-filter computational burden in squint-mode scenarios was calculated to be as little as $5.5 \%$ over that of broadside, with all other imaging parameters remaining equal. (Chapter 6 ). 


\subsection{Future Work}

As with any interesting, challenging and unique endeavor, this research builds a pathway to other interesting and challenging problems. This section provides suggestions for extending this research, thereby improving the utility of the space-variant postfilter as well as furthering its scope of application.

\section{- Parallelization:}

An active area of research in SAR is the high-speed (real-time) formation of spotlightmode imagery via parallel processing. To prevent the space-variant post-filter from becoming the bottleneck in parallel computer implementations of polar-formatting. the post-filtering algorithm should be parallelized, as well. As previously mentioned, post-filtering is based on an analytic expression for quadratic phase error as a function of spatial scene location, and has no dependence on the visual contents of the scene. Furthermore, the filter is one-dimensional in cross-range. Thus, it lends itself nicely to parallelization, given data-domain decomposition in range. In fact, this type of algorithm is the easiest to implement in parallel, and thereby considered embarrassingly parallel.

- Space-variant filter overlap and displacement:

In Chapter 5, relations were developed for determining the optimal filter displacement $d$, overlap $c$ and length $m$ in terms of the floating-point operations count (flops) per pixel, with respect to a specific image location $\left(x_{0}^{\prime}, y_{0}^{\prime}\right)$. Subsequently, in Chapter 6 , the overall computational burden for space-variant post-filtering was calculated based on the optimal values of $d, c$ and $m$ at the worst-case spatial location for defocus. That is, once chosen for worst-case defocus compensation, these parameters were fixed and applied throughout the image, although defocus was typically less severe elsewhere. This provided a straightforward approach for calculating the overall 
computational burden for post-filtering. However, while this simplified the analysis of the computational burden in this dissertation, in practice, the computational burden can be further reduced via space-variant adjustment of $d, c$ and $m$ as a function of spatial image location. This is easily done, given that the analytic expressions for these parameters are already represented as functions of image location.

- Higher-order phase error compensation:

Given the current state of SAR technology, the residual, higher-order phase errors that are left uncompensated upon post-filtering are sufficiently small in magnitude as not to induce visible image defocus. In the future, as radar systems achieve ever higher resolutions, at some point it may become necessary to compensate the third-order (and perhaps higher) phase errors arising from wavefront curvature. As has been demonstrated here for quadratic phase errors, the higher-order correction would require the calculation of subsequent Taylor series terms of the equation for the phase return from a point target. At this point, it remains unknown as to whether these higher-order phase terms will remain one-dimensional in cross-range. If the filtering problem becomes two dimensional, the computational burden will increase significantly.

- Compensation of residual phase pistoning effect for IFSAR:

It has been determined, as a rule of thumb, that a residual quadratic phase $\dot{\phi}_{2 t}$ of less than $\pi / 4$ radians is not sufficiently large to induce visually perceptible defocus in the formed (and magnitude-detected) imagery. That is, given this upper limit for residual quadratic phase, defocus is constrained to within subpixel levels (as shown in Appendix A). Consequently, as a matter of computational efficiency, the spacevariant post-filter is varied only as often as necessary to constrain the defocus to subpixel levels. However, when these post-filtered images are used in their complex (phase and magnitude) form for creating interferometric SAR (IFSAR) products, 
the residual phase errors become significant. In the generation of IFSAR height map products from the complex-valued input images, these phase errors manifest themselves as phase discontinuities which induce "jumps" in the calculated terrain height at post-filter boundaries. These jumps, represented as steps (up or down) in height across the elevation maps, are collectively known as the pistoning effect. This pistoning effect is found in other space-variant operations dealing with image phase, such as space-variant autofocus [94]. As was previously done with autofocus, the pistoning effects induced by phase discontinuities must be resolved before IFSAR processing can be applied to imagery that has been post-filtered for wavefront curvature correction.

- Alternate focus planes, three-dimensional platform motion and arbitrary flight paths:

The phase models for wavefront curvature described herein have been simplified as to convey only the desired scientific principles, without introducing additional complicating issues. However, to apply these principles to practical, real-world imaging scenarios, the models need to be further expanded to improve their versatility. For example, the phase models (and associated equations) should be extended to apply to alternate focus planes in addition to the slant plane scenarios described in this dissertation. In this way, the post-filter can be applied to ground plane imagery, for example, which is considered to provide a visual perspective which is better suited to human interpretation.

This research included the development of a generalized phase model which assumed a linear platform trajectory. This yielded an analytic, closed-form solution for quadratic phase error, strictly as a function of spatial image location and without regard to individual aperture positions. This linear flight path assumption lends to a greatly reduced computational burden compared to that of a formulation requiring phase correction at each position along the flight path. This flight path assumption is restrictive yet not unrealistic, as it models the ISAR scenario of the tomographic 
paradigm, whereby the remote sensing platform remains stationary while the targets to be imaged rotate within the illuminated field. Similarly, a circular flight path could have been chosen, or an elliptical, or any alternative flight path that could be mathematically described, and the phase equations subsequently re-derived to reflect the chosen flight path for the model. However, in real-world scenarios, arbitrary flight paths, or at least those affected by uncompensated motion errors, are a common occurrence. At the very least, the space-variant filter should be suitably modified to accommodate out-of-plane motion, via a three-dimensional extension of the current model. For optimal versatility, the wavefront curvature compensation could be extended to apply to phase histories generated by flying an arbitrary flight path. In this case, the flight path is specified by a set of spatial coordinates (pointing vectors) which describe the arbitrary motion. For this scenario, it is mathematically feasible to compensate the wavefront curvature defocus. However, for arbitrary motion, it is unknown as to whether the computational efficiency could be maintained at the current level. 
Chapter 7. Conclusion

This page intentionally left blank 


\section{Appendix A}

\section{IPR Broadening Due to Quadratic}

\section{Phase Errors}

The impulse response function (IPR) is used to describe the radar system's response to a single isolated point target return and is described mathematically by the dirac delta function $g(u)=\delta(u)$, arbitrarily located at $u=0$. The system is necessarily bandwidth limited and the IPR is a sinc function response. The spatial bandwidth for a spotlight-mode collection is given by

$$
\Delta U_{y}=\Delta Y^{\prime}=\frac{2}{c}\left(2 \pi B_{c}\right) \quad \text { (range extent) }
$$

and

$$
\Delta U_{x}=\Delta X^{\prime}=2\left(\frac{4 \pi}{\lambda}\right) \sin (\Delta \theta / 2) \quad \text { (azimuth extent), }
$$

where $B_{c}$ is the bandwidth of the linear FM chirp launched by the radar at a center frequency of $\omega_{0}, \Delta \theta$ is the angular diversity of the synthetic aperture, and $\lambda$ is the signal wavelength at $\omega_{0}$. Since the angular diversity $\Delta \theta$ is typically very small in spotlight-mode SAR collections, the small angle approximation $\sin (\theta)=\theta$ can be applied to Equation (A.2), yielding

$$
\Delta U_{x} \approx \frac{4 \pi}{\lambda} \Delta \theta
$$


The prime $\left({ }^{\prime}\right)$ in $\Delta X^{\prime}$ and $\Delta Y^{\prime}$ signify that these frequency extents apply to the slant plane reconstruction as opposed to the ground plane projection. The IPR reconstruction consists of a mainlobe and associated sidelobes with the first zero crossings occurring at a distance of $u= \pm \frac{2 \pi}{\Delta U}$, as shown in Figure A.1. A full derivation of Equations (A.1) and (A.2) can be found in [7, pp. 22-24, 72-74]. Since resolution refers to the ability to distinguish between two adjacent IPRs, one can assume they must be separated by at least the distance $u$. In fact, while this simplistic assumption does provide a certain useful and practical measure of resolution, it does not take into account the relative phases of the reflected point target signals, which can further degrade resolution. Another commonly used measure of resolution $\rho$ is the half-power width of the ideal response function such that in general,

$$
\rho=\frac{\pi}{\Delta U}
$$

and specifically,

$$
\rho_{y^{\prime}}=\frac{\pi}{\Delta U_{y}}=\frac{\pi}{Y_{1}^{\prime}}
$$

and

$$
\rho_{x^{\prime}}=\frac{\pi}{\Delta U_{x}}=\frac{\pi}{X_{1}^{\prime}}
$$

for the range and azimuth resolutions, respectively, where $X_{1}^{\prime}$ and $Y_{1}^{\prime}$ define the maximum frequency extent of the aperture in the slant plane, where $\left(\left|\Delta U_{x}\right| \leq X_{1}^{\prime}\right)$ and $\left(\left|\Delta U_{y}\right| \leq Y_{1}^{\prime}\right)$. Again, this measure is optimistic whenever the relative phases of the reflected point target signals are not considered.

The IPR sidelobes also have an effect on image quality. In SAR imaging, it is desirable to limit the peak sidelobe levels of the IPR because high sidelobes confuse the signatures of complex, closely spaced scattering centers, making visual analysis difficult or confusing. This sidelobe energy is generally concentrated in the range and azimuth directions only, with little diagonal energy present. This is due to a phase history which is typically rectangular in shape (after interpolation), with the 


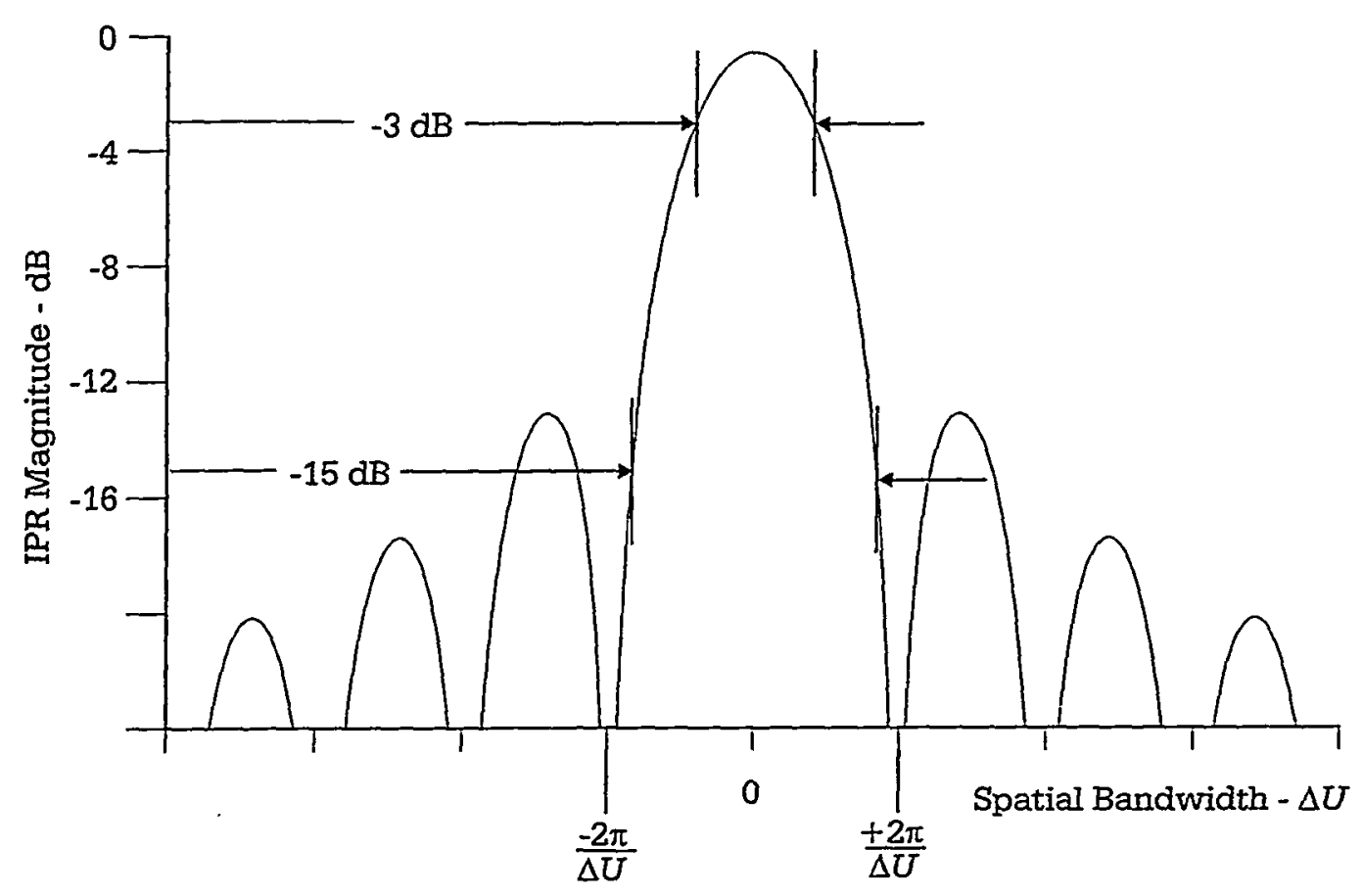

Figure A.1: IPR Response for Point Target with Spatial Bandwidth $\Delta U$

edges oriented in the range and azimuth directions. Furthermore, typical SAR phase errors, which increase sidelobe energy, generally impact phase histories in either the range or azimuth direction with little cross-coupling. However, one exception to the strict range/azimuth orientation of sidlelobes is image formation by convolution back-projection (CBP). This method utilizes the spotlight-mode phase history data in its natural polar annulus form. When these data are exscribed with a rectangular window prior to processing, azimuth-oriented sidelobes are diagonalized with respect to the cross-range axis, at an angle corresponding to the along-track angle subtended by the synthetic aperture. The sidelobe characteristics in imagery constructed using $\mathrm{CBP}$ are discussed in [33]. The natural impact of a limited aperture, as well as the effects of phase errors, require us to maintain a certain tolerance of image degradation due to IPR sidelobes. The sidelobe levels are often reduced or controlled through the use of aperture weighting (windowing) procedures in the range and azimuth directions, as discussed in [12, pp. 507-524], [7, pp. 159-176], [46, pp. 225-239] and 
[77]. However, these techniques generally require a tradeoff between mainlobe width and sidelobe amplitude.

As is the case with many phase errors, the quadratic phase error (QPE) associated with wavefront curvature serves to attenuate the IPR mainlobe while symmetrically increasing sidelobe energy, both of which are detrimental effects. When this phase error is compensated, IPR mainlobe amplitude increases while sidelobe amplitudes decrease, as desired. In considering the degradation of imagery due to quadratic phase errors, the IPR width serves as the metric by which this imagery is judged. While the zero crossing points of the mainlobe may be used as a measure of its width, more commonly the $-3 \mathrm{~dB}$ or $-15 \mathrm{~dB}$ attenuation points are used, as shown in Figure A.1. Thus, by minimizing quadratic phase errors, a maximum acceptable IPR width can be maintained. This generally results in acceptably small sidelobe amplitudes as well, particularly when aperture weighting is incorporated within the image formation process.

The effects of quadratic phase errors on the ideal IPR are shown in Figure A.2. For a phase error of $\phi_{\epsilon} \geq 2 \pi$, which represents a large time-bandwidth product, the sidelobe amplitudes overtake those of the mainlobe. If the $-15 \mathrm{~dB}$ threshold is chosen to measure IPR width, then it is clear that the IPR broadens with increasing quadratic phase error. Just how much IPR broadening occurs at a given QPE? All research to date has relied on empirical data, as shown in Figure A.2, to determine the maximum acceptable quadratic phase error based on the IPR broadening observed. This Appendix serves to provide an analytical approach for relating QPE to IPR broadening. Two different methods are presented, both of which were first proposed in internal company memos [92, 93]. The first considers the the spectral width of the IPR in the frequency domain to determine the broadening factor. The second considers the quadratic shape of the phase error and its rate of change to derive a spatially-based broadening factor, in pixels. As shown, both approaches yield the same result. 
Appendix A. IPR Broadening Due to Quadratic Phase Errors

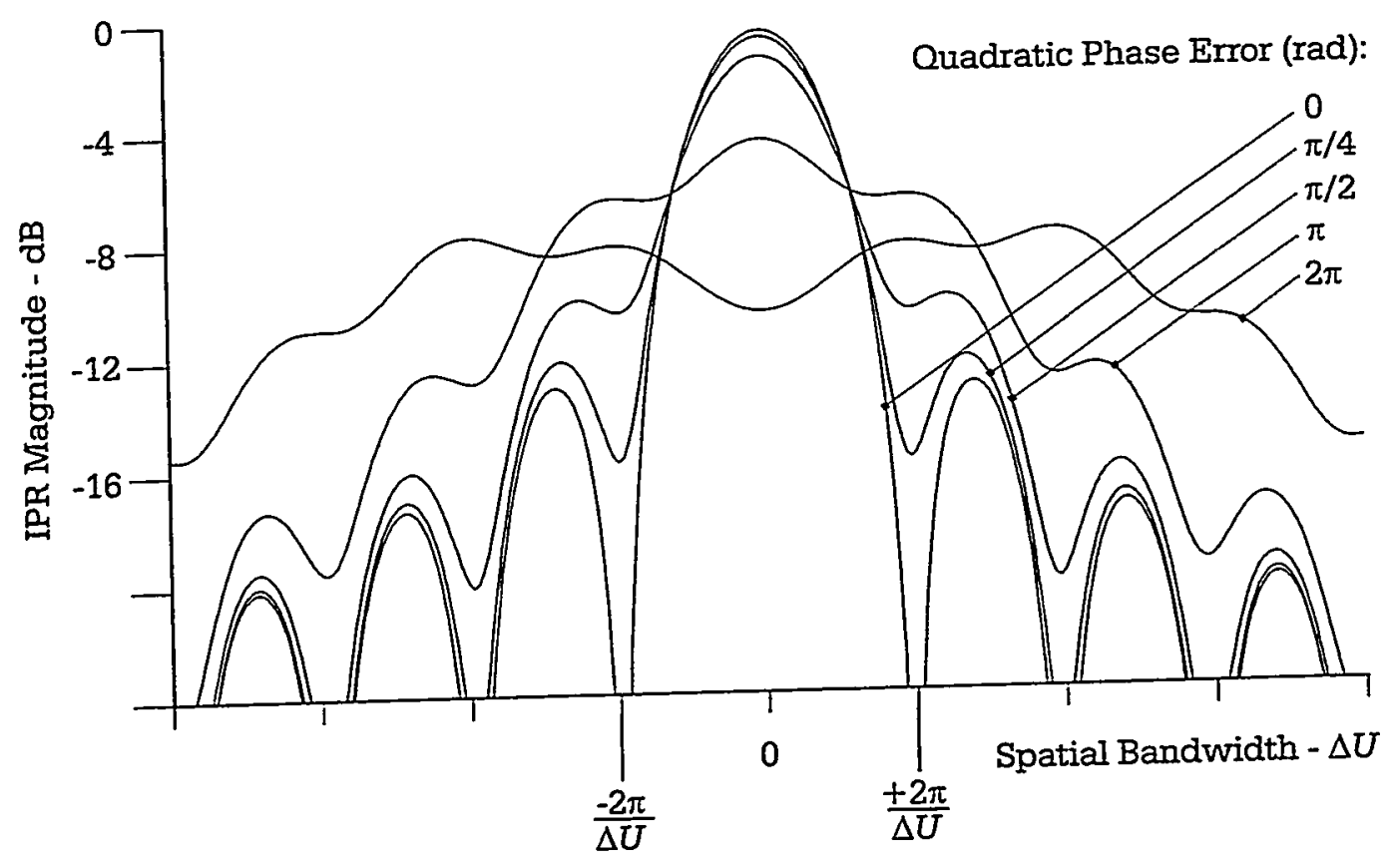

Figure A.2: Effects of Quadratic Phase Errors on the Ideal IPR

\section{A.1 IPR Broadening Based on Spectral Width}

First, consider the following Fourier transform pair, ignoring amplitude factors:

$$
e^{ \pm j \alpha t^{2}} \stackrel{\mathcal{F}}{\Longleftrightarrow} e^{\mp j \frac{\omega^{2}}{4 \alpha}}
$$

where

$$
\begin{aligned}
& \left(T_{1} \leq t \leq T_{2}\right), \\
& \left(\Omega_{1} \leq \omega \leq \Omega_{2}\right),
\end{aligned}
$$

and

$$
\begin{aligned}
& \Omega_{1}= \pm 2 \alpha T_{1}, \\
& \Omega_{2}= \pm 2 \alpha T_{2} .
\end{aligned}
$$


A pulse of duration $\Delta T=T_{2}-T_{1}$ has a nominal spectral width, based on the time-bandwidth product definition, of

$$
\Delta f_{0}=\frac{1}{\Delta T}
$$

For a signal with quadratic phase error $e^{j \alpha t^{2}}$, the nominal spectral width based on Equation (A.7) is

$$
\begin{aligned}
\triangle f_{Q P E} & =\frac{\left(\Omega_{2}-\Omega_{1}\right)}{2 \pi} \\
& =\frac{2 \alpha \Delta T}{2 \pi} \\
& =\frac{\alpha}{\pi} \Delta T .
\end{aligned}
$$

The ratio of quadratic to nominal spectral widths is the broadening factor of the IPR, $\triangle_{I P R}$. Expressing this as a function of $\alpha$ :

$$
\begin{aligned}
\triangle_{I P R} & =\frac{\Delta f_{Q P E}}{\Delta f_{0}} \\
& =\frac{\alpha}{\pi}(\Delta T)^{2} .
\end{aligned}
$$

From the left side of Equation (A.7), the quadratic phase error $\phi_{\epsilon}$ at the signal edge $\Delta T / 2$ is

$$
\begin{aligned}
\phi_{\epsilon} & =\alpha\left(\frac{\Delta T}{2}\right)^{2} \\
& =\frac{\alpha(\Delta T)^{2}}{4} .
\end{aligned}
$$

Solving Equation (A.15) for $\alpha$ in terms of $\phi_{\epsilon}$ yields

$$
\alpha=\frac{4 \phi_{\epsilon}}{(\Delta T)^{2}} \text {. }
$$

Finally, substituting Equation (A.16) into Equation (A.14) and accounting for the possibility of $(\alpha<0)$, yields the IPR broadening factor

$$
\Delta_{I P R}=\frac{4\left|\phi_{\epsilon}\right|}{\pi} \text {. }
$$

For example, given the quadratic phase error $\phi_{\epsilon}=\frac{\pi}{2}$ radians, the IPR broadens by a factor of

$$
\Delta_{I P R}=\left(\frac{4}{\pi}\right) \cdot\left(\frac{\pi}{2}\right)=2
$$




\section{A.2 Spatial Domain IPR Broadening}

An alternative approach considers IPR broadening in terms of the pixel spread in the spatial domain. Consider the quadratic phase error equation

$$
\phi(n)=a\left(n-\frac{N}{2}\right)^{2}, \quad n=0 \ldots N
$$

where $n$ is the sample number, $N$ is the total aperture length in samples, and $a$ is the quadratic coefficient. Let $f_{i}$ be the the instantaneous frequency (in $\mathrm{Hz}$ ) of the phase error at the aperture edges $n=0$ and $n=N$, such that

$$
f_{i n s t}=\left.\frac{1}{2 \pi} \frac{d \phi}{d n}\right|_{n=0: N}
$$

From Equation (A.19),

$$
\begin{aligned}
f_{\text {inst }} & =\left.\frac{1}{2 \pi} 2 a\left(n-\frac{N}{2}\right)\right|_{\substack{n=N \\
n=0}} \\
& = \pm \frac{a N}{2 \pi} .
\end{aligned}
$$

Now, the maximum frequency spread, or spectral width, of this quadratic phase error is

$$
\Delta f_{Q P E}=2\left|f_{i}\right|
$$

and by substitution from Equation (A.21) we have

$$
\triangle f_{Q P E}=\left|\frac{a N}{\pi}\right| .
$$

Analogous to the ratio of spectral widths in the previous case, the pixel spread $\Delta n$ is defined by the following ratio:

$$
\begin{aligned}
\Delta n & =\frac{\text { frequency spread }}{\text { frequency } / \text { pixel }} \\
& =\frac{\Delta f_{Q P E}}{\frac{1}{N}}
\end{aligned}
$$


and by Equation (A.23),

$$
\begin{aligned}
\Delta n & =\frac{\left|\frac{a N}{\pi}\right|}{\frac{1}{N}} \\
& =\frac{|a| N^{2}}{\pi} \text { pixels. }
\end{aligned}
$$

Expressing pixel spread as a function of quadratic phase error at the signal edge requires a substitution for $a$, as follows: First, from Equation (A.19),

$$
\begin{aligned}
\dot{\phi}_{\epsilon} & =\left.\phi(n)\right|_{\substack{n=N \\
n=0}}{ }^{2} \\
& =\left.a\left(n-\frac{N}{2}\right)^{2}\right|_{\substack{n=N \\
n=0}} \\
& =\frac{a N^{2}}{4}
\end{aligned}
$$

for either $n=N$ or $n=0$, which represent the right and left edges of the quadratic phase error function, respectively. Next, solving for $a$,

$$
a=\frac{4 \phi_{\epsilon}}{N^{2}}
$$

and substitution into the pixel spread Equation (A.25) yields

$$
\begin{aligned}
\Delta n_{\epsilon} & =\frac{4\left|\phi_{\epsilon}\right| N^{2}}{\pi N^{2}} \\
& =\frac{4\left|\phi_{\epsilon}\right|}{\pi} \text { pixels. }
\end{aligned}
$$

This result is identical to that of Equation (A.17). 


\section{References}

[1] J. L. Walker, Range-Doppler Imaging of Rotating Objects, Doctoral dissertation, University of Michigan, Ann Arbor, MI, USA, 1974.

[2] J. L. Walker, "Range-doppler imaging of rotating objects," IEEE Transactions on Aerospace and Electronic Systems, vol. AES-16, no. 1, pp. 23-51, January 1980.

[3] J. L. Walker and W. G. Carrara, "Method of processing radar data from a rotating scene using a polar recording format," United States Patent No. 4,191,957, Mar. 31980.

[4] D. C. Munson, J. D. O'Brien, and W. K. Jenkins, "A tomographic formulation of spotlight-mode synthetic aperture radar," Proceedings of the IEEE, vol. 71, no. 8, pp. 917-925, Aug. 1983.

[5] D. A. Ausherman, A. Kozma, J. L. Walker, H. M. Jones, and E. C. Poggio, "Developments in radar imaging," IEEE Transactions on Aerospace and Electronic Systems, vol. AES-20, no. 4, pp. 363-400, July 1984.

[6] C. V. Jakowatz, Jr. and P. A. Thompson, "A new look at spotlight-mode synthetic aperture radar as tomography: Imaging three-dimensional targets," IEEE Transactions on Image Processing, vol. 4, no. 5, pp. 699-703, May 1995.

[7] C. V. Jakowatz, Jr., D. E. Wahl, P. H. Eichel, D. C. Ghiglia, and P. A. Thompson, Spotlight-Mode Synthetic Aperture Radar: A Signal Processing Approach, Kluwer Academic Publishers, Boston, MA, USA, 1996.

[8] W. M. Brown, "SAR resolution in the presence of phase errors," IEEE Transactions on Aerospace and Electronic Systems, vol. AES-24, no. 6, pp. 808-814, Nov. 1988.

[9] A. W. Doerry, "Patch diameter limitations due to high chirp rates in focused synthetic aperture radar images," IEEE Transactions on Aerospace and Electronic Systems, vol. AES-30, no. 4, pp. 1125-1129, Oct. 1994. 
[10] C. V. Jakowatz, Jr., D. E. Wahl, P. A. Thompson, and N. E. Doren, "Spacevariant filtering for correction of wavefront curvature effects in spotlight-mode SAR imagery formed via polar formatting," in Proceedings of the SPIE, Orlando, FL, USA, April 23-24 1997, The International Society for Optical Engineering; vol. 3070 of Algorithms for Synthetic Aperture Radar Imagery IV, pp. 33-42.

[11] N. E. Doren, C. V. Jakowatz, Jr., D. E. Wahl, and P. A. Thompson, "General formulation for wavefront curvature correction in polar-formatted spotlightmode SAR images using space-variant post-filtering," in Proceedings of the International Conference on Image Processing, Santa Barbara, CA, USA, Oct. 1997, IEEE Signal Processing Society, vol. 1, pp. 861-864.

[12] W. G. Carrara, R. S. Goodman, and R. M. Majewski, Spotlight Synthetic Aperture Radar-Signal Processing Algorithms, Artech House, Boston, MA, USA, 1995.

[13] B. McArthur and K. Kaufman, "A generalized polar processing algorithm for large area SAR images," in Proceedings of the 1991 IEEE National Radar Conference, Los Angeles, CA, USA, Mar. 1991, Institute of Electrical and Electronic Engineers, pp. 83-87.

[14] F. Rocca, C. Cafforio, and C. Prati, "Synthetic aperture radar: A new application for wave equation techniques," Geophysical Prospecting, vol. 7, no. 37, pp. 809-830, 1989.

[15] C. Cafforio, C. Prati, and F. Rocca, "SAR data focusing using seismic migration techniques," IEEE Transactions on Aerospace and Electronic Systems, vol. 27, no. 2, pp. 194-206, Mar. 1991.

[16] C. Prati, A. M. Guarnieri, and F. Rocca, "Spot mode SAR focusing with the $\omega-K$ technique," in Proceedings of the 1991 IEEE Interenational Geoscience and Remote Sensing Symposium (IGARSS), Espoo, Finland, June 1991, IEEE Geoscience and Remote Sensing Society, vol. 306, pp. 631-634.

[17] C. Prati and F. Rocca, "Focusing SAR data with time-varying doppler centroid," IEEE Transactions on Geoscience and Remote Sensing, vol. 30, no. 3, pp. 550-559, May 1992.

[18] A. Golden, Jr., S. C. Wei, K. K. Ellis, and S. Tumala, "Migration processing of spotlight SAR data," in Proceedings of the SPIE, Orlando, FL, USA, April 6-7 1994, The International Society for Optical Engineering, vol. 2230 of Algorithms for Synthetic Aperture Radar Imagery, pp. 25-35.

[19] T. J. Flynn, "Wavenumber-domain SAR focusing from a nonuniform synthetic aperture," in IEEE 1992 International Conference on Acoustics, Speech and 
Signal Processing (ICASSP-92). Institute of Electrical and Electronic Engineers, Mar. 1992, vol. 3, pp. I-4.

[20] J. L. H. Webb and D. C. Munson Jr., "SAR image reconstruction for an arbitrary radar path," in IEEE 1995 20th International Conference on Acoustics, Speech and Signal Processing (ICASSP-95), Detroit, MI, USA, Mar. 5-12 1995, Institute of Electrical and Electronic Engineers, vol. 4, pp. 2285-2288.

[21] H. Runge and R. Bamler, "A novel high precision SAR focussing algorithm based on chirp scaling;" in Proceedings of the 1992 IEEE Interenational Geoscience and Remote Sensing Symposium (IGARSS). IEEE Geoscience and Remote Sensing Society, May 26-29 1992, vol. 1, pp. 372-375.

[22] R. K. Raney, "An exact wide field digital imaging algorithm," International Journal of Remote Sensing, vol. 13, no. 5, pp. 991-998, May 1992.

[23] R. K. Raney, H. Runge, R. Bamler, I. G. Cumming, and F. H. Wong, "Precision SAR processing using chirp scaling," IEEE Transactions on Geoscience and Remote Sensing, vol. 32, no. 4, pp. 786-799, July 1994.

[24] M. Y. Jin, F. Cheng, and M. Chen, "Chirp scaling algorithms for SAR processing," in Proceedings of the 1993 IEEE Interenational Geoscience and Remote Sensing Symposium (IGARSS), Tokyo, Japan, Aug 1993, IEEE Geoscience and Remote Sensing Society, pp. 1169-1172.

[25] A. Moreira and Y. Huang, "Airborne SAR processing of highly squinted data using a chirp scaling approach with integrated motion compensation," IEEE Transactions on Geoscience and Remote Sensing, vol. 32, no. 5, pp. 1029-1040, Sept. 1994.

[26] X. Qiu and Z. Zhu, "Super-resolution imaging used in chirp scaling algorithm," in Proceedings of the IEEE 1996 National Aerospace and Electronics Conference (NAECON), Dayton, OH., USA, May 20-23 1996, Institute of Electrical and Electronic Engineers, vol. 1, pp. 355-358.

[27] D. W. Hawkins, "An accelerated chirp scaling algorithm for synthetic aperture imaging," in Proceedings of the 1997 IEEE Interenational Geoscience and Remote Sensing Symposium (IGARSS), Singapore, Aug. 3-8 1997, IEEE Geoscience and Remote Sensing Society, vol. 1 of A Scientific Vision for Sustainable Development, pp. 471-473.

[28] G. W. Davidson, Image Formation From Squint Mode Synthetic Aperture Radar Data, Ph.D. thesis, The University of British Columbia, Vancouver, Canada, Sept. 1994. 
[29] F. Impagnatiello, "A precision chirp scaling SAR processor extension to subaperture implementation on massively parallel supercomputers," in Proceedings of the 1995 IEEE Interenational Geoscience and Remote Sensing Symposium (IGARSS), Firenze, Italy, July 10-14 1995, IEEE Geoscience and Remote Sensing Society, vol. 3 of Quantitative Remote Sensing for Science and Applications, pp. 1819-1821.

[30] J. L. Bauck and W. K. Jenkins, "Tomographic processing of spotlight-mode synthetic aperture radar signals with compensation for wavefront curvature;" in ICASSP 88: 1988 International Conference on Acoustics, Speech and Signal Processing, New York, NY, USA, April 11-14 1988, IEEE Signal Processing Society, vol. 2, pp. 1192-1195.

[31] J. L. Bauck and W. K. Jenkins, "Convolution-backprojection image reconstruction for bistatic synthetic aperture radar with correction for wavefront curvature and propagation attenuation," in Proceedings of the SPIE, Orlando, FL, USA, March 27-28 1989, The International Society for Optical Engineering, vol. 1101 of Millimeter Wave and Synthetic Aperture Radar, pp. 11-18.

[32] J. L. Bauck, Tomographic Processing of Synthetic Aperture Radar Signals for Enhanced Resolution, Doctoral dissertation, University of Illinois at UrbanaChampaign, Ürbana, IL, USA, Jan. 1990.

[33] C. D. Knittle, N. E. Doren, and C. V. Jakowatz, Jr., "A comparison of spotlight synthetic aperture radar image formation techniques," Sandia Report SAND962460, Sandia National Laboratories, P.O. Box 5800, Albuquerque, NM., 87185, Oct. 1996.

[34] J. W. Adams, R. W. Bayma, M. E. Lawrence, and L. Petrosian, "On the modified chirp $Z$ transform for synthetic aperture radar systems," IEEE Transactions on Signal Processing, vol. 39, no. 4, pp. 953-955, Apr. 1991.

[35] R. Lanari, "A short discussion on the exact compensation of the SAR rangedependent range cell migration effect," IEEE Transactions on Geoscience and Remote Sensing, vol. 35, no. 6, pp. 1446-1452, Nov. 1997.

[36] G. A. Mastin, S. J. Plimpton, and D. C. Ghiglia, "A massively-parallel digital processor for spotlight synthetic-aperture radar," International Journal of Supercomputer Applications, vol. 7, no. 2, pp. 97-112, Summer 1993.

[37] G. Fabbretti, A. Farina, D. Laforenza, and F. Vinelli, "Mapping the synthetic aperture radar signal processor on a distributed-memory MIMD architecture," Parallel Computing, vol. 22, no. 5, pp. 761-784, Aug. 1996.

[38] P. G. Meisl, M. R. Ito, and I. G. Cumming, "Parallel synthetic aperture radar processing on workstation networks:" in Proceedings of the 10th International 
Parallel Processing Symposium (IPPS). Institute of Electrical and Electronic Engineers, April 15-19 1996, pp. 716-723.

[39] G. Franceschetti, A. Mazzeo, N. Mazzocca, V. Pascazio, and G. Schirinzi, "An efficient SAR parallel processor," IEEE Transactions on Aerospace and Electronic Systems, vol. 27, no. 2, pp. 343-352, Mar. 1991.

[40] G. A. Mastin, S. J. Plimpton, and D. C. Ghiglia, "Massively parallel syntheticaperture radar autofocus," in Proceedings of the SPIE. The International Society for Optical Engineering, Dec. 1991, vol. 1566 of Advanced Signal Processing Algorithms, Architectures, and Implementations II, pp. 341-352.

[41] B. Walker, G. Sander, M. Thompson, B. Burns, R. Fellerhoff, and D. Dubbert, "A high-resolution, four-band SAR testbed with real-time image formation," in Proceedings of the 1991 IEEE Interenational Geoscience and Remote Sensing Symposium (IGARSS). IEEE Geoscience and Remote Sensing Society, May 1996, vol. 3, pp. 1881-1885.

[42] M. Medina and N. Magotra, "Implementation of a subaperture image formation," in Ideas In Science and Electronics, Albuquerque, NiM, USA, May 1989, ISE Oral Sessions, Ideas In Science and Electronics Symposium (ISE).

[43] B. L. Burns and J. T. Cordaro, "A SAR image-formation algorithm that compensates for the spatially-variant effects of antenna motion," in Proceedings of the SPIE. The International Society for Optical Engineering, June 1994, vol. 2230 of Algorithms for Synthetic Aperture Radar Imagery, pp. 14-24.

[44] B. L. Burns and J. T. Cordaro, "Imaging synthetic aperture radar," United States Patent No. 5,608,404, Mar. 31997.

[45] A. W. Doerry, "Synthetic aperture radar processing with polar formatted subapertures," in Proceedings of the 28th Asilomar Conference on Signals, Systems and Computers, Pacific Grove, CA, USA, Oct. 31 - Nov. 2 1994, Asilomar.

[46] A. W. Doerry, Synthetic Aperture Radar Processing With Tiered Subapertures, Doctoral dissertation, University of New Mexico, Albuquerque, NM, USA, May 1995.

[47] A. W. Doerry and N. Magotra, "Wide angle fine resolution spotlight SAR with tiered subapertures," Sandia Report SAND95-2284J, Sandia National Laboratories, P.O. Box 5800, Albuquerque, NM., 87185, Oct. 1995.

[48] I. A. Erteza, "Parallel implementation of the polar-format algorithm using MPI," To be published as technical SAND report, Sandia National Laboratories, Albuquerque, NM, USA, 87185, TBD 2000. 
[49] D. A. Ausherman, "SAR digital image-formation processing," in Proceedings of the SPIE, Los Angeles, CA, USA, Jan. 22-23 1985, The International Society for Optical Engineering, vol. 528 of Digital Image Processing, pp. 118-133.

[50] A. M. Smith, "A new approach to range-Doppler SAR processing," International Journal of Remote Sensing, vol. 12, no. 2, pp. 235-251, 1991.

[51] J. P. Fitch, Synthetic Aperture Radar, Springer-Verlag, New York, NY, USA, 1988.

[52] L. J. Cutrona, W. E. Vivian, E. N. Leith, and G. O. Hall, "A high resolution radar combat surveillance system," IRE Transactions on Military Electronics, vol. MIL-5, pp. 127-131, 1961.

[53] R. Lipps and D. Kerr, "Polar reformatting for ISAR imaging;" in RADARCON '98: Proceedings of the 1998 IEEE Radar Conference, Dallas, TX., USA, May 11-14 1998, pp. 275-280, Institute of Electrical and Electronic Engineers.

[54] M. S. R. Rao and P. R. Mahapatra, "Synthetic aperture radar: A focus on current problems," Defence Science Journal, vol. 47, no. 4, pp. 517-536, Oct. 1997.

[55] J. D. O'Brien, A Consideration of Signal Processing for Spotlight Synthetic Aperture Radar; Doctoral dissertation, University of Illinois, Urbana-Champaign, Urbana, IL, USA, 1995.

[56] M. Soumekh, Synthetic Aperture Radar Signal Processing with MATLAB Algorithms, John Wiley and Sons, New York, NY, USA, 1999.

[57] J. C. Curlander and R. N. McDonough, Synthetic Aperture Radar - Systems and Signal Processing, John Wiley and Sons, New York, NY, USA, 1991.

[58] A. W. Rihaczek, Synthetic Aperture Radar - Systems and Signal Processing, Peninsula Publishing, Los Altos, CA, USA, 1985.

[59] N. Levanon, Radar Principles, John Wiley and Sons, New York, NY, USA, 1988.

[60] B. C. Barber, "Theory of digital imaging from orbital synthetic-aperture radar," International Journal of Remote Sensing, vol. 6, no. 7, pp. 1009-1057, July 1985.

[61] D. C. Munson Jr. and R. L. Visentin, "A signal processing view of strip-mapping synthetic aperture radar," IEEE Transactions on Acoustics, Speech, and Signal Processing, vol. 37, no. 12, pp. 2131-2146, Dec. 1989.

[62] P. T. Gough and D. W. Hawkins, "Unified framework for modern synthetic aperture imaging algorithms," International Journal of Imaging Systems and Technology, vol. 8, no. 4, pp. 343-358, 1997. 
[63] S. R. Deans, The Radon Transform and Some of Its Applications, Wiley and Sons, New York, NY, USA, 1983.

[64] L. C. Graham, "Synthetic interferometer radar for topographic mapping;" Proceedings of the IEEE, vol. 62, no. 6, pp. 763-768, June 1974.

[65] P. H. Eichel, D. C. Ghiglia, C. V. Jakowatz, Jr., P. A. Thompson, and D. E. Wahl, "Spotlight SAR interferometry for terrain elevation mapping and interferometric change detection," Sandia Report SAND93-2072, Sandia National Laboratories, P.O. Box 5800, Albuquerque, NM., 87185, Oct. 1993.

[66] D. C. Munson and J. L. C. Sanz, "Image reconstruction from frequency-offset Fourier data," in Proceedings of the IEEE. Institute of Electrical and Electronic Engineers, June 1984, vol. 72, pp. 661-669.

[67] W. Lawton, "A new polar fourier transform for computer-aided tomography and spotlight synthetic aperture radar," IEEE Transactions on Acoustics, Speech, and Signal Processing, vol. 36, no. 6, pp. 931-933, June 1988.

[68] E. B. Champagne, "A revisit to spotlight processing," in Proceedings of Radarcon '90: First Australian Radar Conference, Adelaide, Australia, April 18-20 1990, vol. 2, pp. 353-358, Defense Science and Technology Organization, Australia.

[69] E. D. Banta, "Limitations on SAR image area due to motion through range cells," IEEE Transactions on Aerospace and Electronic Systems, vol. AES-22, no. 6, pp. 799-805, Nov. 1986.

[70] D. A. Schwartz, "Analysis and experimental investigation of three synthetic aperture radar formats," M.S. Thesis (Coordinated Science Lab - tech. rep. t-94), University of Illinois, Urbana, IL, USA, Mar. 1980.

[71] M. D. Desai and W. K. Jenkins, "Convolution backprojection image reconstruction for spotlight mode synthetic aperture radar," IEEE Transactions on Image Processing, vol. 1, no. 4, pp. 505-517, Oct. 1992.

[72] J. Mann, "Spotlight-mode synthetic aperture radar without fourier transforms," in Proceedings of the SPIE. The International Society for Optical Engineering, June 1994, vol. 2230 of Algorithms for Synthetic Aperture Radar Imagery, pp. 2-13.

[73] W. K. Jenkins, "New techniques in digital signal processing," Grant AFOSR-790029, Report no. AFOSR-TR-81-0321, for Air Force Office of Scientific Research, Bolling AFB, DC, USA, University of Illinois, Coordinated Science Lab, Feb. 1981. 
[74] G. Wolberg, Ditigal Image Warping, IEEE Computer Society Press Monograph. IEEE Computer Society Press, Los Alamitos, CA, USA, 1990.

[75] B. C. Mather, Fourier Domain Interpolation Techniques for Synthetic Aperture Radar, Doctoral dissertation, University of Illinois, Urbana, IL, USA, Jan. 1986.

[76] J. W. Adams, R. E. Hudson, R. W. Bayma, and J. E. Nelson, "Digital interpolators for polar format processing," in Proceedings of the SPIE, Orlando, FL, USA, Mar. 27-28 1989, The International Society for Optical Engineering, vol. 1101 of Millimeter Wave and Synthetic Aperture Radar, pp. 34-45.

[77] H. C. Stankwitz, R. J. Dallaire, and J. R. Fienup, "Nonlinear apodization for sidelobe control in SAR imagery," IEEE Transactions on Aerospace and Electronic Systems, vol. 31, no. 1, pp. 267-279, Jan. 1995.

[78] P. H. Eichel, D. C. Ghiglia, and C. V. Jakowatz, Jr., "Speckle processing method for synthetic aperture radar phase correction," Optics Letters, vol. 14, Jan. 1989.

[79] D. E. Wahl, P. H. Eichel, D. C. Ghiglia, and C. V. Jakowatz, Jr., "Phase gradient autofocus - a robust tool for high resolution SAR phase corrections," IEEE Transactions on Aerospace and Electronic Systems, vol. 30, no. 3, pp. 827-834, July 1994.

[80] T. M. Calloway and G. W. Donohoe, "Subaperture autofocus for synthetic aperture radar," IEEE Transactions on Aerospace and Electronic Systems, vol. 30, no. 2, pp. 617-621, Apr. 1994.

[81] D. R. Sheen, T. B. Lewis, S. C. Wei, and D. Kletzli, Jr., "Imaging of buried and foliage-obscured objects with an ultrawide bandwidth polarimetric SAR," in Proceedings of the SPIE, Orlando, FL, USA, 1992, The International Society for Optical Engineering, vol. 1942, pp. 12-20.

[82] D. R. Sheen, C. M. Strawitch, and T. B. Lewis, "UHF wideband SAR design and preliminary results," in Proceedings of the 1994 IEEE Interenational Geoscience and Remote Sensing Symposium (IGARSS), Pasadena, CA, USA, Aug. 1994, IEEE Geoscience and Remote Sensing Society, pp. 289-291.

[83] R. Goodman, S. Tummala, and W. Carrara, "Issues in ultra-wideband, widebeam SAR image formation," in Record of the 1995 IEEE International Radar Conference, Alexandria, VA, USA, May 8-11 1995, Institute of Electrical and Electronic Engineers, pp. 479-485.

[84] T. H. Cormen, C. E. Leiserson, and R. L. Rivest, Introduction to Algorithms, MIT Press, Cambridge, MA, USA, 1992.

[85] G. Franceschetti and R. Lanari, Synthetic Aperture Radar Processing, CRC Press, Boca Raton, FL, USA, 1999. 
[86] K. K. Kong and J. A. Edwards, "Polar format blurring in ISAR imaging," IEE Electronics Letters, vol. 3121, no. 17, pp. 1502-1503, Aug. 171995.

[87] N. Brousseau, "Focusing properties of synthetic aperture radar interferograms having range curvature aberrations," Applied Optics, vol. 18, no. 15, pp. 25802585, Aug. 1979.

[88] M. Jin and C. Wu, "SAR correlation technique - an algorithm for processing data with large range walk," in Proceedings of the 1997 IEEE Interenational Geoscience and Remote Sensing Symposium (IGARSS '83), San Francisco, CA, USA, Aug. 31-Sep.2 1983, IEEE Geoscience and Remote Sensing Society, vol. 2, pp. FA-3.1.1-FA-3.1.5.

[89] S. Wang and X. Huang, "Autofocus techniques for reducing phase errors in UWB-SAR," in Proceedings of the IEEE 1997 National Aerospace and Electronics Conference (NAECON), Dayton, OH., USA, July 14-17 1997, Institute of Electrical and Electronic Engineers, vol. 2, pp. 1009-1014.

[90] R. E. Blahut, Fast Algorithms for Digital Signal Processing, Addison-Wesley, Reading, MA, USA, 1985.

[91] A. W. Doerry, "Shaving Ops in OSA processing;" Internal company memorandum, Sandia National Laboratories, Albuquerque, NM, USA, Aug. 81996.

[92] D. C. Ghiglia, "IPR spread in pixels as a function of quadratic phase error," Internal company memorandum, Sandia National Laboratories, Albuquerque, NM. USA, Mar. 1991.

[93] D. C. Ghiglia, "Alternative derivation of IPR broadening by quadratic phase errors," Internal company memorandum, Sandia National Laboratories, Albuquerque, NM, USA, July 1994.

[94] D. E. Wahl, "Space-variant autofocus," Internal company memorandum, Sandia National Laboratories, Albuquerque, NM, USA, Apr. 251996. 
References

This page intentionally left blank 


\section{Distribution}

2 MS 1207 C.V. Jakowatz, 5912

11207 D.E. Wahl, 5912

11207 P.A. Thompson, 5912

$11207 \quad$ I.A. Erteza, 5912

101207 N.E. Doren, 5912

10529 B.C. Walker, 2308

$10978 \quad$ L.G. Stotts, 5934

10529 A.W. Doerry, 2345

$10537 \quad$ T.J. Flynn, 2344

19018 Central Technical Files, 8940-2

20899 Technical Library, 4916

10612 Review and Approval Desk, 4912

For DOE/OSTI

10161 Patent and Licensing Office, 11500 